\title{
Management of late preterm prelabour rupture of membranes
}

Citation for published version (APA):

van der Ham, D. P. (2013). Management of late preterm prelabour rupture of membranes. [Doctoral Thesis, Maastricht University]. Maastricht University. https://doi.org/10.26481/dis.20131002dh

Document status and date:

Published: 01/01/2013

DOI:

10.26481/dis.20131002dh

Document Version:

Publisher's PDF, also known as Version of record

\section{Please check the document version of this publication:}

- A submitted manuscript is the version of the article upon submission and before peer-review. There can be important differences between the submitted version and the official published version of record.

People interested in the research are advised to contact the author for the final version of the publication, or visit the DOI to the publisher's website.

- The final author version and the galley proof are versions of the publication after peer review.

- The final published version features the final layout of the paper including the volume, issue and page numbers.

Link to publication

\footnotetext{
General rights rights.

- You may freely distribute the URL identifying the publication in the public portal. please follow below link for the End User Agreement:

www.umlib.nl/taverne-license

Take down policy

If you believe that this document breaches copyright please contact us at:

repository@maastrichtuniversity.nl

providing details and we will investigate your claim.
}

Copyright and moral rights for the publications made accessible in the public portal are retained by the authors and/or other copyright owners and it is a condition of accessing publications that users recognise and abide by the legal requirements associated with these

- Users may download and print one copy of any publication from the public portal for the purpose of private study or research.

- You may not further distribute the material or use it for any profit-making activity or commercial gain

If the publication is distributed under the terms of Article $25 \mathrm{fa}$ of the Dutch Copyright Act, indicated by the "Taverne" license above, 


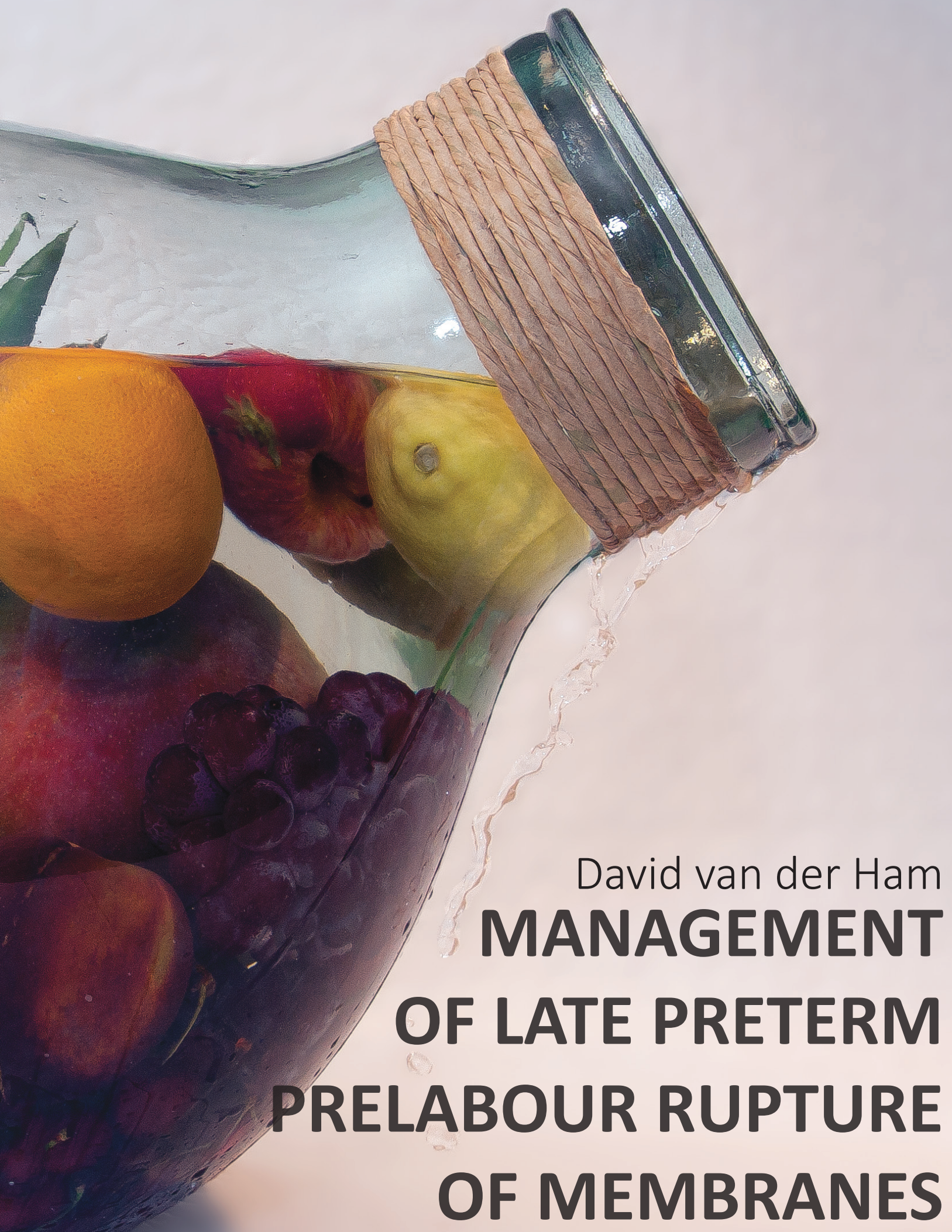





\title{
MANAGEMENT OF LATE PRETERM PRELABOUR RUPTURE OF MEMBRANES
}

\author{
DAVID P VAN DER HAM
}


The studies presented in this theses were supported by the Dutch Organisation for Health Research and Development, ZonMW (grant 94507212)

The author gratefully acknowledges financial support for printing this thesis by:

RVE Obstetrie \& Gynaecologie - Martini Ziekenhuis Groningen

Wetenschapsbureau Martini Ziekenhuis

Merck Sharp \& Dohme B.V.

Noord Negentig Accountants en Belastingadviseurs

Ferring B.V.

BMA B.V. (Mosos)

Hologic Netherlands B.V.

Johnson \& Johnson Medical B.V.

Memidis Pharma B.V.

Mölnlycke Health Care B.V.

Olympus Nederland B.V.

Medical Dynamics B.V.

ISBN: 978-94-6191-824-6

Cover: "Vruchtwater" - photo by D.P. van der Ham

Layout: D.P. van der Ham

Print: Ipskamp Drukkers B.V. Enschede

(C) Copyright 2013 David P van der Ham, the Netherlands

All right reseverd. No part of this thesis may be reproduced, stored in a retrieval system, or transmitted in any form or by any means, without prior permission of the author. 


\section{MANAGEMENT OF LATE PRETERM PRELABOUR RUPTURE OF MEMBRANES}

\section{PROEFSCHRIFT}

ter verkrijging van de graad van doctor aan de Universiteit Maastricht, op gezag van de Rector Magnificus,

Prof.dr. L.L.G. Soete

volgens het besluit van het College van Decanen,

in het openbaar te verdedigen

op woensdag 2 oktober 2013 om 12:00 uur

door

David Pelaja van der Ham

Geboren 15 november 1978

te Groningen 


\section{PROMOTORES}

Prof. Dr. J.G. Nijhuis

Prof. Dr. B.W.J. Mol, AMC Amsterdam

\section{COPROMOTORES}

Dr. C. Willekes

Dr. A.L.M. Mulder

\section{BEOORDELINGSCOMMISSIE}

Prof. Dr. C.A..Bruggeman, voorzitter

Dr. P.H. Dijk, UMCG Groningen

Prof. Dr. J.A.M. van der Post, AMC Amsterdam

Prof. Dr. F.C.S. Ramaekers

Prof. Dr. L.J.I. Zimmerman 



\section{TABLE OF CONTENT}

\begin{tabular}{ll} 
ChAPTER 1 & General Introduction \\
\hline
\end{tabular}

ChAPTER 2 Methods for the diagnosis of rupture of the fetal membranes in equivocal cases: A systematic review

Eur J Obstet Gynecol Reprod Biol.

CHAPTER 3 Prelabour rupture of membranes: overview of diagnostic

methods

Curr Opin Obstet Gynecol.

CHAPTER 4 Accuracy of C-reactive protein determination in predicting chorioamnionitis and neonatal infection in pregnant women with premature rupture of membranes: A systematic review Eur J Obstet Gynecol Reprod Biol.

CHAPTER 5 Study protocol: Induction of labour versus expectant management in women with preterm prelabour rupture of membranes between 34 and 37 weeks (the PPROMEXIL-trial) BMC Pregnancy Childbirth.

CHAPter 6 Induction of labor versus expectant management in women with preterm prelabor rupture of membranes between 34 and 37 weeks: A randomized controlled trial PLoS Med.

CHAPTER 7 Management of late-preterm premature rupture of membranes: the PPROMEXIL-2 trial Am J Obstet Gynecol.

ChAPTER 8 Neonatal outcome of pregnancy complicated by PPROM between 34 and 37 weeks of gestation Submitted 
CHAPTER $9 \quad$ Neonatal sepsis in women with PPROM between 34 and 37

weeks of gestation: development of a prediction model

Submitted

ChaPter 10 Economic analysis comparing induction of labour and expectant management in women with preterm prelabour rupture of membranes between 34 and 37 weeks (PPROMEXIL trial)

Submitted

Chapter 11 General discussion

Summary/Nederlandse samenvatting

Appendix

List of co-authors and collaborators

Acknowledgments

References

Publications and presentations

Dankwoord

Curriculum vitae 



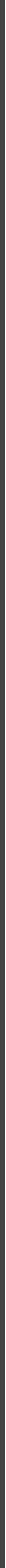




\section{CHAPTER}

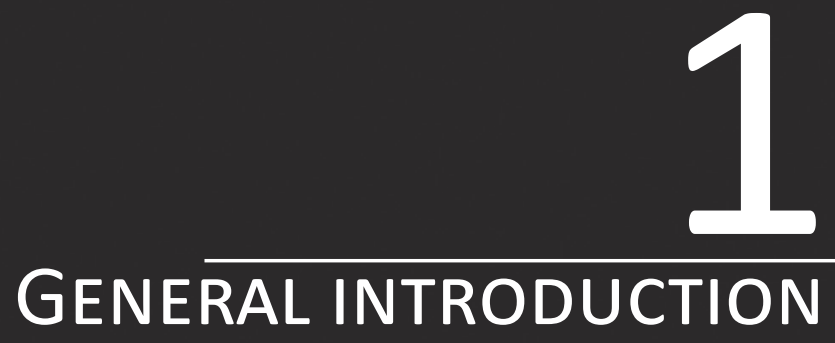




\section{INTRODUCTION}

\section{PRELABOUR RUPTURE OF MEMBRANES}

Rupture of the fetal membranes before onset of labour (PROM - prelabour rupture of membranes) is a frequently encountered obstetrical problem. It complicates approximately $10 \%$ of all births, and is associated with chorioamnionitis and perinatal and maternal morbidity. ${ }^{1,2}$ At term a large randomised study between induction of labour (loL) versus expectant management (EM) with oxytocin or prostaglandin showed that maternal and neonatal outcome were found to be similar between IoL and EM, regardless of augmentation method. ${ }^{3}$ The rate of caesarean section did not differ significantly $10.1 \%$ versus $9.7 \%$ for loL versus EM with oxytocin and $9.6 \%$ versus $10.9 \%$ for loL versus EM with prostaglandin. ${ }^{3}$ The risk of neonatal infection was not significant $2.0 \%$ (loL) versus $2.7 \%$ (EM) for the oxytocin group and $3.0 \%$ (IoL) versus $2.7 \%$ (EM) for the prostaglandin group. However, women, in this study, viewed induction of labour more positively than expectant management. ${ }^{3}$ This finding, however was not confirmed by others. ${ }^{4}$ Nevertheless, at term labour will mostly be induced after PROM within 24 until 72 hours. This policy is also part of the Dutch guideline. ${ }^{5}$

In approximately $3 \%$ of all pregnancies PROM occurs prematurely, $0.5 \%$ of pregnancies before $26^{+0}$ weeks are complicated by PPROM, $1.0 \%$ between $26^{+0}$ and $34^{+0}$ weeks and $1.5 \%$ between $34^{+0}$ and $37^{+0}$ weeks of gestational age. ${ }^{5}$ Premature PROM accounts for $40 \%$ of all preterm births. ${ }^{3,6}$ This means that in The Netherlands approximately 3,000 women per year are admitted to the hospital because of PPROM between $34^{+0}$ and $37^{+0}$ weeks.

\section{RISKS OF PREMATURE PRELABOUR RUPTURE OF MEMBRANES}

Rupture of membranes is associated with several clinical problems such as chorioamnionitis, ascending infection, neonatal infection or sepsis, prematurity, abruption placentae, cord prolapse and possibly increased risk of stillbirth. ${ }^{2,7,8}$

As mentioned near term it is advocated that prolongation of pregnancy no longer benefits the fetus. ${ }^{9}$ However induction of labour might lead to increased risks due to prematurity and a possible rise in the number of instrumental deliveries.

Although PPROM is associated with infectious morbidity, ${ }^{1}$ only a minority of women will suffer from an infection. Little is known on how to identify those women who are at risk. It is, until now, not clear whether the potential beneficial effects of induction of labour will overcome the disadvantageous effects of premature birth, for either mother or child. Therefore premature PROM is still a dilemma for the perinatal team, i.e. obstetrician and neonatologist. 


\section{DIAGNOSING RUPTURE OF MEMBRANES}

In the vast majority of women a diagnosis of ruptured membranes can be made based on a history of a sudden gush of fluid, followed by on-going fluid loss combined with visualisation of amniotic fluid loss by physical examination. In approximately $10 \%$ of the cases the diagnosis is more difficult to make. ${ }^{2,10}$ The conventional testing method based on a combination of medical history, visual pooling at speculum examination, alkaline $\mathrm{pH}$ determination and/or microscopic ferning combined with a decrease of amniotic fluid on ultrasound is prone to a high false positive rate, due to contamination with blood, urine or semen. ${ }^{10-13}$ Furthermore speculum examination is found to be very intrusive by pregnant women. ${ }^{14}$ Although dozens of tests have been introduced since the first description of alkaline testing in the 1930s, international guidelines do not give a clear recommendation on how to diagnose rupture of membranes, especially not in equivocal cases.5,15,16

\section{MANAgeMENT OF PREMATURE PRELABOUR RUPTURE OF MEMBRANES}

International guidelines have different opinions on the treatment of near term PROM. 5,15,16 All guidelines admit that there is a lack of level $1 \mathrm{~A}$ evidence and treatment recommendations are made on small studies. Furthermore different "consensus" opinions have led to different recommendations around the world.

The American Congress of Obstetricians and Gynecologists recommends induction of labour if PPROM occurs at or beyond 34 weeks of gestation. ${ }^{15}$

The Green-Top Guideline of the Royal College of Obstetricians and Gynaecologists states: "Delivery should be considered at 34 weeks of gestation. Where expectant management is considered beyond this gestation, women should be informed of the increased risk of chorioamnionitis and the decreased risk of respiratory problems in the neonate."16

The Dutch Society of Obstetricians and Gynaecologists (NVOG), however, advises: "Expectant management until 35 weeks gestational age, unless maternal or fetal contraindications exists, or develop. From 35 weeks gestational age onwards continuation of expectant management can be discussed with the patient." ${ }^{\prime 5}$

The lack of consensus on the management of women with PROM near term was also addressed by two surveys from Australia and Canada. ${ }^{17,18}$

A recent Cochrane review on the management of PPROM prior to 37 weeks $^{19}$ include seven small studies published between 1981 and 1998. These studies included between 47 and 160 women with a gestational age range between 25 and $36^{+6}$ weeks at the moment of PROM. ${ }^{20-26}$ The included trials are of variable methodological quality. Only one had an adequate allocation concealment, ${ }^{24}$ while none of the trials were blinded for all 
outcome. In only one trial the neonatologists were blinded for the treatment allocation of the women. ${ }^{26}$

The authors of the Cochrane review concluded that there is currently insufficient evidence for the management of PPROM in clinical practice. ${ }^{19}$

In more detail, only one trial in the Cochrane review solely focused on the near term population, ${ }^{24}$ while the others also included women with midtrimester PROM. In this study 120 women with PPROM between $34^{+0}$ and $36^{+6}$ weeks gestational age were included. ${ }^{24}$ Induction of labour led to a nonsignificant decrease in chorioamnionitis without a significant difference in neonatal morbidity. When treated expectantly there was a nonsignificant increase in the risk of neonatal sepsis and length of hospitalization of the newborns. A reduction in the incidence of chorioamnionitis and nonsignificant decrease in neonatal sepsis rate after induction of labour was also found by Mercer et al. in a small trial (93 women) between $32^{+0}$ and $36^{+6}$ weeks. ${ }^{23}$ Spinnato et al. found no difference in neonatal outcome between expected management and prompt delivery in 47 women with PPROM between $25^{+0}$ and $36^{+0}$ weeks with documented fetal pulmonary maturity. ${ }^{26}$ However, they demonstrated an increased risk of maternal infection when expectant management was applied.

In near term PROM small studies show a trend towards a better neonatal outcome when inducing labour however in coincidence with the authors of the Cochrane review, the evidence is insufficient to guide clinical practice for the near term population.

\section{ONSET OF THIS THESIS}

The lack of consensus and evidence on the management of prelabour rupture of membranes in late preterm women was the onset of the Induction of Labour versus EXpectant management in women with Preterm Prelabour Rupture of Membranes between 34 and 37 weeks (PPROMEXIL) trial. ${ }^{27}$

In a multicentre randomised controlled trial we compared induction of labour versus expectant management in women with late preterm PROM in order to improve the evidence and guide clinical practice in these women.

\section{THE AIM OF THE STUDY}

Does induction of labour decrease the incidence of neonatal sepsis without an unacceptable increase in neonatal and maternal morbidity? 


\section{PRIOR TO BELIEF QUESTIONNAIRE}

Prior to the start of the PPROMEXIL trial we performed a prior-to-belief questionnaire. This online questionnaire was sent by e-mail to all members of the Dutch Society of Obstetrics and Gynaecology (NVOG), gynaecologist and residents. The rationale of this questionnaire was to observe what the opinions and believes of gynaecologists were before the start of the PPROMEXIL trial.

\section{OUTCOME MEASUREMENTS}

Gynaecologists were asked what they thought to be the risk on neonatal sepsis when treating a women with PPROM expectantly, whether induction of labour would lead to an increase or decrease of neonatal sepsis. We also asked gynaecologists to estimate the increase/change in maternal complication rate (chorioamnionitis, sepsis, instrumental deliveries) when they would treat a woman expectantly and whether induction of labour would increase or decrease the risk of maternal complications. Finally we asked what they thought to be the risk of a vaginally assisted delivery or a Caesarean Section (CS) when labour was induced.

We stated that the occurrence of a serious neonatal complication (e.g. neonatal sepsis, severe respiratory distress syndrome, need of ventilation) was considered to be more severe than a CS. We then asked how many CS they were prepared to perform to prevent one serious neonatal complication.

We asked to estimate the increased risk for respiratory distress when labour was induced, and similar to above which ratio they would consider to be acceptable for respiratory distress against neonatal sepsis.

We investigated current practice among respondents and asked their opinion about the estimated effect size of the PPROMEXIL study in order to change their opinion of their current management. We finally asked their believes in the effect size of maternal and neonatal morbidity when labour is induced in case of PPROM.

\section{RESULTS}

In total 236 (20\%) gynaecologists and residents responded to the questionnaire.

\section{Practice before PPROMEXIL}

Eighty two per cent of responders indicated that women were treated expectantly in their institutions, in $11 \%$ women were induced and in $6.2 \%$ there was no preference. When we asked whether the gynaecologists (or residents) own opinion was to induce women with 
PPROM between 34 and 37 weeks, 38\% agreed to induce labour and 42\% disagreed, 20\% did not favour either policy.

\section{Estimated risks}

The estimated median risk for neonatal sepsis was 5\% (Inter quartile range [IQR] 4-10\%) with a very wide range (0\% to $70 \%)$. In total $88 \%$ of the responders expected a decrease in the risk of neonatal sepsis when labour was induced. Fifty nine per cent believed that induction of labour should lead to a decline in maternal morbidity.

The estimated median risk for maternal complication was 10\% (IQR 5-15\%), whereas the median risk for a vaginally assisted delivery or CS was 20\% (IQR 15-30\%) and 10\% (IQR 5-15\%) for RDS.

If asked how many CS may be performed to prevent one serious adverse neonatal outcome the median outcome was $10 \mathrm{CS}$ to prevent 1 serious adverse neonatal event (IQR 4-20). Table 1.1 summarises the expected effect size of induction of labour in the most important neonatal and maternal outcome of the PPROMEXIL trial.

\begin{tabular}{lccccc} 
& $\begin{array}{c}\text { Large } \\
\text { reduction }\end{array}$ & $\begin{array}{c}\text { Small } \\
\text { reduction }\end{array}$ & $\begin{array}{c}\text { No } \\
\text { difference }\end{array}$ & $\begin{array}{c}\text { Small } \\
\text { increase }\end{array}$ & $\begin{array}{c}\text { Large } \\
\text { increase }\end{array}$ \\
\hline Neonatal outcome & & & & & \\
\hline Neonatal sepsis & $29(15 \%)$ & $149(75 \%)$ & $16(0.8 \%)$ & $3(1.5 \%)$ & $2(1.0 \%)$ \\
\hline $\begin{array}{l}\text { Late onset sepsis } \\
\text { Respiratory distress }\end{array}$ & $13(6.5 \%)$ & $130(65 \%)$ & $52(26 \%)$ & $3(1.5 \%)$ & $1(0.5 \%)$ \\
syndrome & $0(0 \%)$ & $11(5.5 \%)$ & $22(11 \%)$ & $158(79 \%)$ & $8(4.0 \%)$ \\
\hline $\begin{array}{l}\text { Other pulmonary problems } \\
\text { Necrotising enterocolitis }\end{array}$ & $0(0 \%)$ & $12(6.1 \%)$ & $60(30 \%)$ & $122(61 \%)$ & $4(2.0 \%)$ \\
\hline $\begin{array}{l}\text { Hypoglycaemia } \\
\text { Maternal outcome }\end{array}$ & $0(0 \%)$ & $11(5.6 \%)$ & $98(50 \%)$ & $87(45 \%)$ & $2(1.0 \%)$ \\
\hline Haemorrhage & $0(0 \%)$ & $22(11 \%)$ & $130(64 \%)$ & $51(25 \%)$ & $0(0 \%)$ \\
\hline $\begin{array}{l}\text { Cord Prolaps } \\
\text { Maternal infection }\end{array}$ & $1(0.5 \%)$ & $50(25 \%)$ & $106(53 \%)$ & $45(22 \%)$ & $0(0 \%)$ \\
\hline Maternal sepsis & $12(11 \%)$ & $145(72 \%)$ & $22(11 \%)$ & $13(6.4 \%)$ & $1(0.5 \%)$ \\
\hline Chorioamnionitis & $43(21 \%)$ & $134(66 \%)$ & $49(24 \%)$ & $8(3.9 \%)$ & $0(0 \%)$ \\
\hline Endometritis & $18(9.0 \%)$ & $137(68 \%)$ & $36(18 \%)$ & $10(5.0 \%)$ & $0(0 \%)$ \\
\hline
\end{tabular}

Assumed effect of induction of labour in women with preterm prelabour rupture of membranes between 34 and 37 weeks amongst 236 gynaecologists and residents OBGYN in a prior-to-belief questionnaire before the onset of the PPROMEXIL trials.

a Percentages given are related to available data per characteristic

Tabel 1.1 | Assumed effect of induction of labour for different neonatal and maternal outcomes 


\section{COMMENT}

Although the response to the questionnaire was low and the opinions amongst the responders varied wide, it gives a fair impression of the opinions prior to the start of the PPROMEXIL trial.

Interestingly the very wide range of estimated outcome and thus the believes or gut feeling of different professionals for that certain outcome might influence their policy probably more than solid evidence. This finding is concerning. An implementation study of several completed studies of the Dutch Consortium for Women's health and reproductivity studies (www.studies-obsgyn.nl) is currently planned and undertaken to study the implementation of the results of these studies in daily practice. 


\section{OUTLINE OF THE THESIS}

In $10 \%$ of the cases rupture of membranes is difficult to diagnose. In Chapter 2 the results of a systematic review on the diagnostic tests for diagnosing ruptured membranes in equivocal cases are presented. Chapter $\mathbf{3}$ is a complement to Chapter $\mathbf{2}$ in which the latest developments in diagnostic tests on ruptured membranes and are discussed. In order to identify women with a higher risk for developing an infectious complication, especially neonatal sepsis, after PROM the level of C-reactive protein is of potential use. In Chapter $\mathbf{4}$ the results of a systematic review on the diagnostic accuracy of C-reactive protein on the prediction of neonatal sepsis and/or clinical/histological chorioamnionitis are presented.

Chapter 5 outlines the study protocol of the PPROMEXIL trial. In Chapter 6 the results of the first PPROMEXIL trials are presented. Due to a lower than expected incidence of neonatal sepsis the PPROMEXIL trial was underpowered and in view of uncertainty of the continuation of another large on-going trial on the subject an additional trial was performed to increase the level of evidence. The results of this trial (PPROMEXIL-2) are presented in Chapter 7.

Chapter 8 presents the nationwide results, based on the perinatal registry (PRN) of 2,670 women with PPROM for a least 24 hours between 34 and 37 weeks from 2000 to 2006. Because of the rather expectant treatment policy throughout The Netherlands these results mimic the outcome of expectant management. Due to the size of the database which include more than 1,4 million deliveries we were able to estimate the risk of stillbirth in women with PROM (> 24 hour) from 34 weeks onwards, compared to controls with intact membranes.

It seems possible, based on antepartum parameters, to ascertain the risk of neonatal sepsis for each individual woman, which might help the clinician in decision making. In Chapter 9 the results of this prediction model based on the results of both PPROMEXIL trials are presented.

Chapter 10 shows the economic analysis of both treatment strategies of the PPROMEXIL trial.

Chapter 11 discusses the management of late preterm prelabour rupture of membranes by evaluation the results of the both PPROMEXIL trials, secondary analysis and retrospective studies. It further discusses the limitations of current large randomised controlled trials in national as well as global perspective and set out new perspectives for national and global collaboration in future research. 



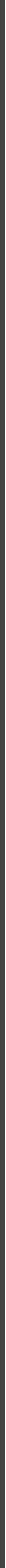




\title{
CHAPTER
}

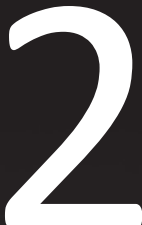

\section{METHODS FOR THE DIAGNOSIS OF RUPTURE OF THE FETAL MEMBRANES IN EQUIVOCAL CASES: A SYSTEMATIC REVIEW}

\author{
David P van der Ham, Marjo JGJ van Melick, Luc Smits, Jan G Nijhuis, \\ Carl P Weiner, J (Hans) J van Beek, Ben Willem J Mol, Christine Willekes
}

Eur J Obstet Gynecol Reprod Biol. 2011 Aug;157(2):123-7. Epub 2011 Apr 8 


\begin{abstract}
Prelabor rupture of membranes (PROM) is a common obstetrical problem, but its diagnosis is frequently problematic. Lacking a gold standard, the diagnosis is equivocal in some $10 \%$ of cases. We performed a systematic review to assess the accuracy of several tests for the diagnosis of PROM in these equivocal cases. We performed an electronic search in PubMed, Embase, DARE and the Cochrane Library and reference lists for potentially missed articles. No language restrictions were used. Only accuracy studies for diagnostic methods for PROM in women with equivocal PROM were selected. The studies were scored according to STARD and QUADAS guidelines. Based on the full description of reference and index tests, an expert panel finally decided whether the selected articles were of sufficient quality to be included. We identified 3864 studies of which 146 full manuscripts were obtained. We excluded 133 due to multiple reasons. The remaining 13 studies were scored by an expert panel. Only three articles with a total of 155 patients fulfilled all criteria. These articles tested three different methods, $\mathrm{pH}$ measurement (64 patients), insulin-like growth factor binding protein-1 (ILGBP-1, 83 patients) and alpha fetoprotein (AFP, 8 patients). Sensitivity varied from $88 \%$ (pH) to $100 \%$ (AFP), specificity varied from $56 \%$ (ILGPP-1) to $100 \%$ (AFP). Based on the limited evidence on the accuracy of tests to diagnose ruptured membranes, we conclude that the use of a particular test cannot be recommended.
\end{abstract}




\section{INTRODUCTION}

Prelabor rupture of the fetal membranes (PROM) is a frequently encountered obstetrical problem. PROM complicates $5-10 \%$ of all pregnancies. ${ }^{28,29}$ It occurs in $1-5 \%$ of preterm pregnancies, accounting for $30-40 \%$ of all preterm deliveries. ${ }^{30-32}$ PROM is associated with an increased incidence of chorioamnionitis and prematurity and with increased perinatal and maternal morbidity and mortality. ${ }^{1}$ In the majority of women, the diagnosis of rupture of the fetal membranes can be confirmed based on a suspicious history and unequivocal loss of amniotic fluid. In approximately $10 \%$ of all cases, however, the diagnosis of rupture of membranes is difficult to establish. ${ }^{2,10}$

Since the introduction of alkaline testing of the vaginal fluid in the 1930s as a test for amniotic fluid loss, ${ }^{33}$ dozens of tests have been proposed for the identification of loss of amniotic fluid. Initially, these tests also focused on the perinate's cells, like vernix caseosa cells, fetal fat particles or lanugo hairs. ${ }^{10}$ For decades, a combination of visual pooling of amniotic fluid during speculum examination, alkaline $\mathrm{pH}$ determination and microscopic evidence of ferning has been widely used to determine rupture of membranes. Because of the need for a speculum examination, many women find this very intrusive. ${ }^{14}$ Furthermore, these tests are prone to false positive results secondary to vaginal contamination with blood, urine, or semen. ${ }^{10}$

Other diagnostic parameters have been proposed to simplify test procedures and to reduce false positive rates, including alpha-fetoprotein (AFP), ${ }^{34,35}$ beta-HCG, ${ }^{36,37}$ fetal creatinine, ${ }^{38,39}$ diamine oxidase activity (DAO), ${ }^{40,41}$ ferning, ${ }^{11}$ fetal fibronectin (fFN) ${ }^{42,43}$ glucose/fructose determination, ${ }^{44}$ insulin-like growth factor binding protein 1 (ILGFBP-1), ${ }^{45,46}$ interleukin 6 (IL-6), ${ }^{34}$ lactate, ${ }^{47,48}$ Nile Blue sulfate, ${ }^{13,49}$ panty-liner $\mathrm{pH}$ determination, ${ }^{50,51}$ phenazopyridine hydrochloride, ${ }^{52}$ pinacyanole, ${ }^{10}$ placental alpha microglobulin-1 (PAMG$1)^{53,54}$ and prolactin..$^{5,56}$

Theoretically an intra-amniotic cavity infusion of mock amniotic fluid containing a dye would be the gold standard for the diagnosis of rupture of membranes, but the procedure is invasive and may itself cause rupture of membranes. Depending on the dye selected (e.g. methylene blue), complications like hemolytic jaundice, hemolytic anemia, hyperbilirubinemia, and methemoglobinemia may occur. ${ }^{50,57-60}$ The use of phenol-sulfonphthalein (PSP) ${ }^{58}$ or indigo carmine ${ }^{15}$ is clinically safe, however. Nevertheless there is a strong need for a reliable, and preferably, noninvasive test.

No previous study has systematically reviewed the available evidence concerning the performance of prevailing tests for premature rupture of membranes. In the present review, we summarize and critically evaluate published evidence on this subject. In our opinion there is no need for a diagnostic test in women with unequivocal ruptured membranes, 
but when this is doubtful, there is a need for a highly sensitive and specific diagnostic test. Because missing a diagnosis of ROM may lead to an increased risk of neonatal sepsis, however, wrongfully inducing labor because of a false positive diagnosis may lead to maternal and neonatal complications, especially when induced prematurely. Therefore we decided to limit our target

population to women with equivocal rupture of membranes. Only studies including such women were considered eligible for this review. We applied criteria for the methodological assessment and reporting of diagnostic accuracy studies adapted from guidelines suggested previously. ${ }^{61-64}$

\section{MethodS}

\section{SEARCH STRATEGY}

We searched PubMed (1966-09/2010), EMBASE (1974-09/2010), the Cochrane Library, DARE and CENTRAL for relevant papers. Reference lists of all primary articles were examined to identify cited articles not found in the initial electronic search. In PubMed, we used a search strategy for studies evaluating diagnostic accuracy, ${ }^{62}$ combined with MeSH terms for specific diagnostic tests, using Boolean operators "AND" and "OR" as appropriate. We combined this with subheadings and MeSH terms on rupture of membranes. This search strategy was adapted for the other databases. There were no language restrictions. Selected papers in languages other than English, German, French or Dutch, were translated by medically qualified professionals unaffiliated to this study.

Eligible papers were selected in a four-stage process. First, two independent reviewers $(\mathrm{DH}, \mathrm{MM})$ carried out the electronic search. Both scrutinized all titles and/or abstracts for eligible studies. Second, full manuscripts were obtained for all studies selected by at least one of the reviewers. Reference lists of all primary articles were examined to identify cited articles not found in the initial electronic search. Third, all remaining articles were assessed in a panel discussion (DH, MM, LS, CW) before inclusion. Articles were discussed and scored based on selection criteria as well as QUADAS and STARD guidelines for appropriateness and study quality. Finally, an expert panel (BM, CW, JJB) judged whether, in the absence of a perfect gold standard, the description of the reference test used was likely to identify the target condition (rupture of membranes) as suggested by Rutjes et al. ${ }^{15}$

\section{SELECTION CRITERIA}

We included only those studies in which one or more diagnostic tests were intended to identify rupture of membranes in women with clinical signs suggestive of rupture of membranes but in whom the diagnosis rupture of membranes was equivocal. If a study 
population consisted of both equivocal and unequivocal rupture of membranes, it was only included if a subgroup analysis of women with equivocal rupture of membranes was delineated. Case studies were excluded, and patient selection had to be prospective. Case control studies were excluded because of the risks of overestimating sensitivity and specificity. ${ }^{62}$ Inclusion and/or exclusion criteria had to be described in sufficient detail. All participants had to undergo the same reference and index test. The index test had to be described in sufficient detail, or at least a reference providing a full description for the test method had to be available. An expert panel (BM, CW, JJB), blinded for the study results, decided if the description of the reference test used was likely to identify rupture of membranes. ${ }^{63}$ Both the index test and the reference test had to be classified as positive (ruptured membranes) or negative (no ruptured membranes).

Only studies that fulfilled all criteria described above were included in this review.

\section{DATA EXTRACTION AND SYNTHESIS}

Data extraction was performed using a data extraction form according to the QUADAS and STARD criteria. ${ }^{61,64}$ Our intention was to construct one or more $2 \times 2$ tables for each study, cross classifying the index test on ruptured membranes against the reference test.

\section{RESULTS}

\section{SELECTION PROCESS}

Figure 2.1 illustrates the selection process. From the initial 3864 articles, we selected 146 full manuscripts after reading the abstract. Of these 146 articles, 133 studies were excluded for miscellaneous reasons outlined in figure 2.1. Thirteen studies passed the first three stages of the selection process. . $11,43,45,46,50,53,58,65-70$

\section{EXPERT PANEL}

The expert panel discussed each study thoroughly before deciding on inclusion or exclusion. Five studies $43,46,65,68,69$ were excluded because of an inadequate description of the reference test used. As an example, Mulhair et al. described their reference test ("golden standard") as: "The attending midwife performed a sterile speculum examination to detect rupture of membranes". ${ }^{68}$ Five studies were excluded because their study population mixed equivocal and unequivocal rupture of membranes without a possibility of separation. ${ }^{11,50,53,66,67}$ Cousins et al. described their study population as "pregnant women with signs and/or symptoms of membrane rupture were invited to participate in the study". ${ }^{3}$ In our opinion, this might include patients with equivocal as well as unequivocal rupture of membranes. Likewise, Bornstein et al. included "pregnant women reporting a feeling 


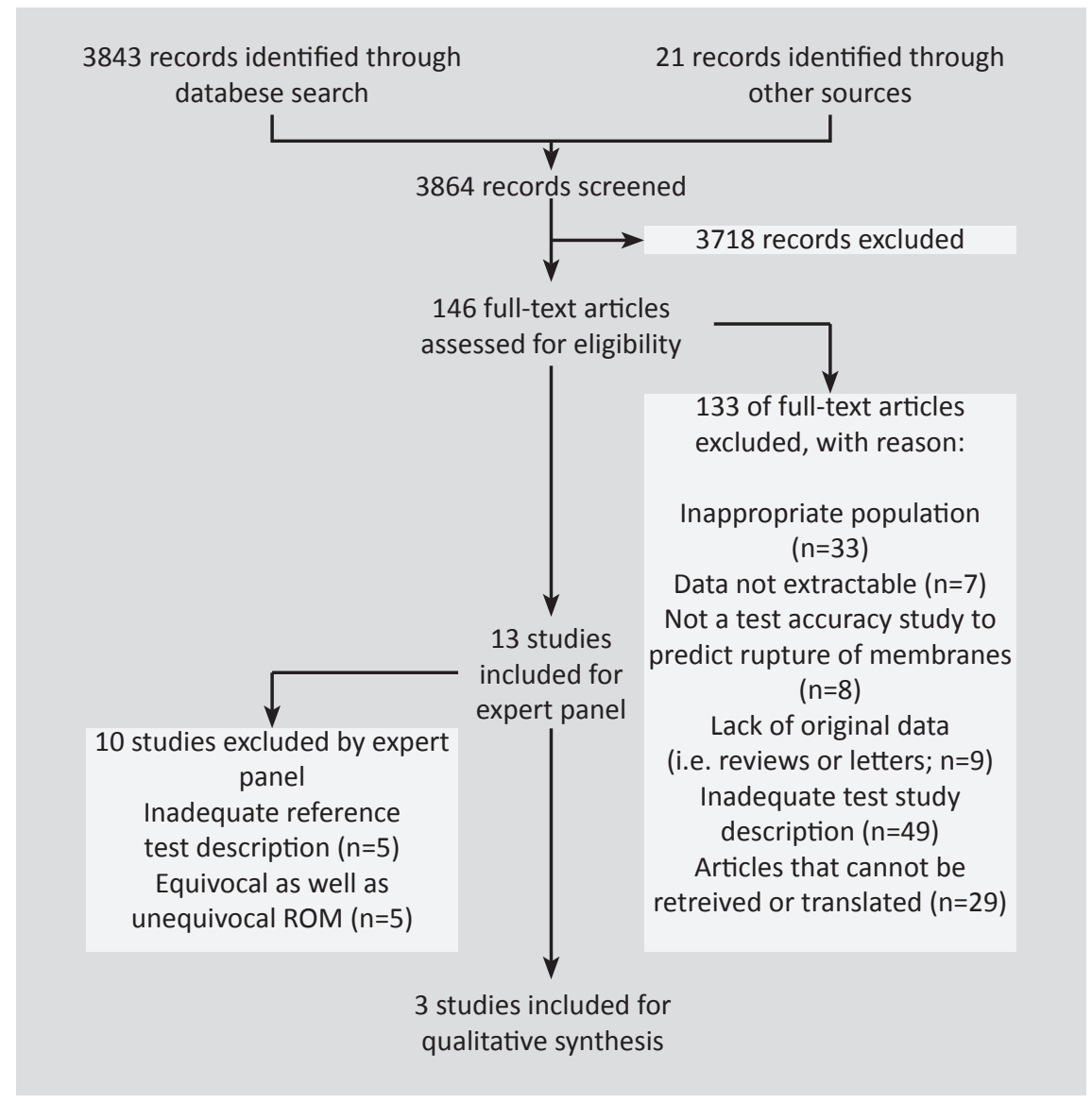

Figure 2.1 | Study selection process

of vaginal wetness (undetermined if due to amniotic fluid leakage or urine) were eligible to participate in the study. Women with gross rupture of membranes were excluded, women in active labor were not excluded". 50

\section{INCLUDED STUDIES}

The study characteristics of the remaining three studies are summarized in Table 2.1. Table 2.2 shows the test methods used, with their cut-off values and sensitivity and specificity. Fujimoto et al. ${ }^{58}$ and Yamada et al. ${ }^{70}$ used as their gold standard amniocenteses with dye. Jeurgens-Borst et al. ${ }^{45}$ used a retrospective evaluation to consider the status of the fetal membranes at the moment of testing.

All test methods were only described once. Sensitivity and specificity varied among the tests from 87 to $100 \%$ and 56 to $100 \%$ respectively. 


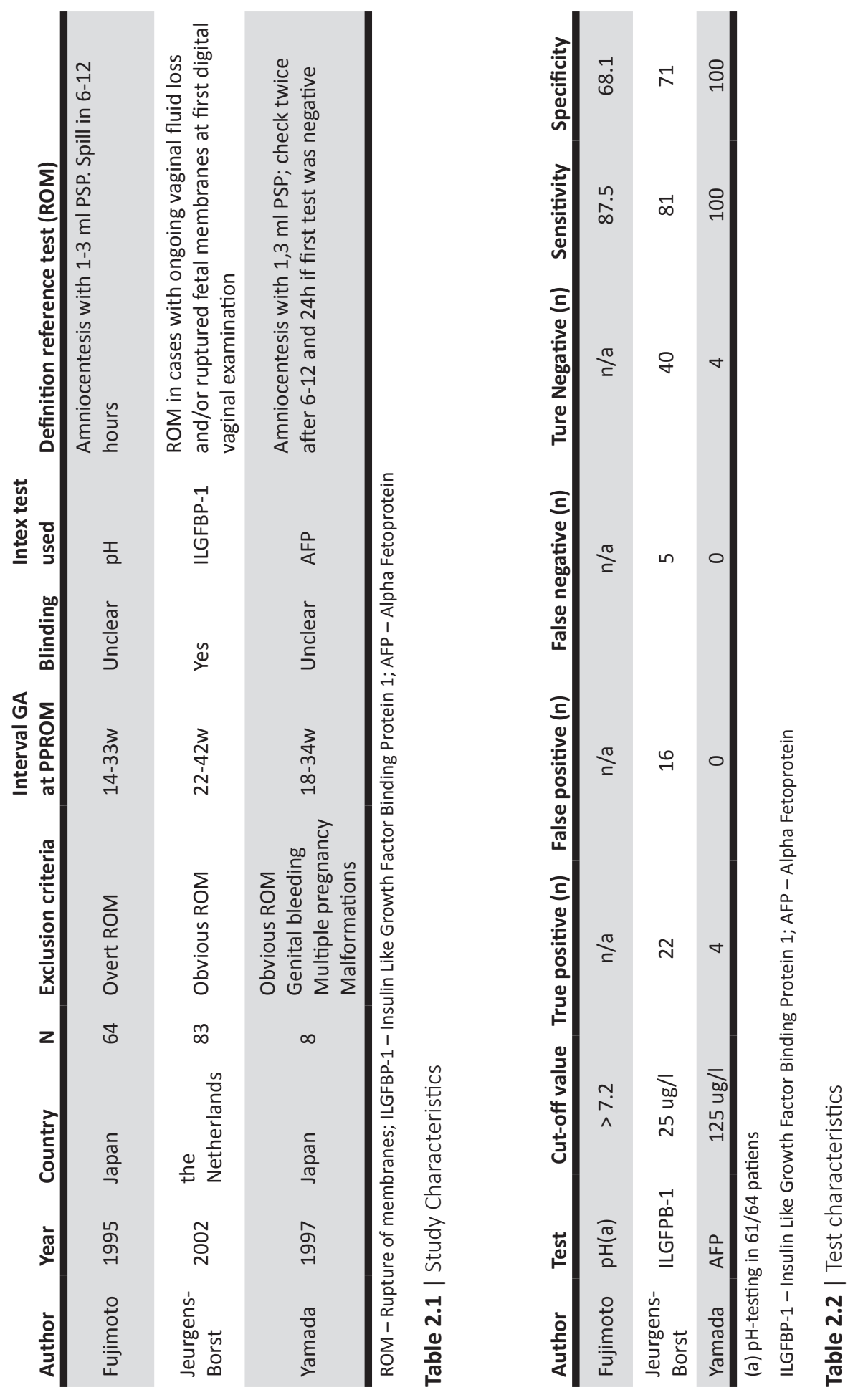


Fujimoto et al. used an intra-amniotic infusion of phenol-sulfonphthalein (PSP) in women with equivocal premature rupture of membranes. Over a 12-year period they diagnosed premature rupture of membranes with the PSP method in 64 women. In addition to this test, they performed $\mathrm{pH}$ measurements, the choline esterase test and the ferning test. Only the $\mathrm{pH}$ measurement was described in sufficient details to be included in this review. $\mathrm{pH}$ test was only performed in 61 (of 64) women and the authors presented percentages instead of absolute numbers. Therefore we were not able to calculate the absolute numbers of true-positive and true-negative women. They reported a sensitivity of $87.5 \%$ and a specificity of $68.1 \%$, without any confidence interval. ${ }^{58}$

For the choline esterase test, a reference was made to an achol test method without any description of a cut-off value, nor was a reference mentioned. This test was performed in 42 of 64 women. For the ferning test they did not describe the time for drying the samples or the microscopic settings used. They performed the ferning test in 36 of 64 patients. Jeurgens-Borst et al. used a predefined reference test. Fetal membranes were considered ruptured in cases with ongoing vaginal fluid loss and/or ruptured fetal membranes at first digital vaginal examination. Jeurgens-Borst used this method as their "gold standard". IGFBP-1 had a lower sensitivity and specificity in comparison to the clinical diagnosis made by the attending obstetrician at the moment of testing ( $81 \%, 71 \%$ vs. $85 \%$. $79 \%$ respectively). Confidence intervals were reasonably small (0.63-0.92 for sensitivity, and $0.59-0.82$ for specificity). ${ }^{45}$

Yamada et al. reported a sensitivity and specificity for AFP of $100 \%$ using PSP amniotic cavity infusion as the gold standard, but the $95 \%$ confidence interval was very wide (51$100 \%$ ) due to low numbers of patients (eight in total). Gestational age ranged from $18^{+1}$ weeks to $30^{+6}$ weeks in the negative group and $30^{+4}$ weeks to $34^{+2}$ in the positive group. ${ }^{70}$

\section{COMment}

Due to an initially broad search strategy we found a large number of over 3800 potential papers. After scanning titles we could reduce this to 146 manuscripts, but only three studies fulfilled our predefined selection criteria. The main reasons for exclusion were inadequate test or study description and inadequate study population. The vast majority of qualitatively adequate studies examined amniotic fluid testing in either an amniotic fluid sample, collected after artificial rupture of membranes or amniocentesis, or in women with gross loss of fluid at speculum examination. The three remaining studies included 155 women and covered three different test methods. Although there was agreement among the expert panel to include these three studies, the quality was not optimal in all aspects. All studies included women with equivocal rupture of membranes at very 
early gestational ages, starting from 14 to 22 weeks of gestational age. It is reasonable to assume that the composition of early amniotic fluid may well differ from that later in pregnancy.

Fujimoto et al. needed a 12-year period to include 64 women. ${ }^{58}$ Although they used the golden standard their description of the diagnostic test used was poor. Not all women underwent all tests and it was not clear whether one or more observers performed the tests. One might debate the retrospective method used by Jeurgens-Borst et al. ${ }^{45}$ The low specificity and sensitivity (56 and $89 \%$ respectively) of IGFBP-1 might be related to a less adequate reference test. Jeurgens-Borst et al. described a predefined retrospective reference test, which was determined without knowledge of the results of the IGFBP-1 test. Our expert panel, blinded for study results, considers this as an appropriate manner to tackle the problem without amniotic cavity infusion, and we therefore included this study. In contrast, other studies with retrospective reference tests had either inadequate study

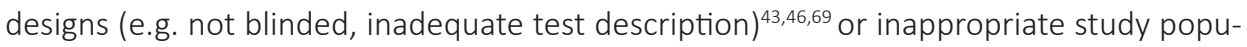
lations. ${ }^{11,66}$

Yamada et al. reported a high sensitivity and specificity of $100 \%$ each. ${ }^{70}$ AFP was only tested in eight women, however, rendering wide 95\%-confidence intervals (51-100) around both estimates.

The lack of qualitatively good studies in women with equivocal rupture of membranes raises the question how one can design a study that meets modern standards. According to the ACOG and others, one might consider a protocol in which all available diagnostic methods are tested against the true gold standard: ultrasonographically guided transabdominal instillation of indigo carmine dye..$^{2,15,58}$ This, however, might lead to an ethical discussion whether or not to expose a patient to such an invasive procedure.

This systematic review could not reveal which diagnostic test(s) should be used in patients with unequivocal rupture of membranes. Based on this systematic review, we suggest rather than deciding on one single diagnostic test to determine whether or not a patient has rupture of membranes in equivocal cases, to reconsider the diagnosis over a couple of hours and if necessary days. According to logistics and gestational age one might decide to admit the patient to the hospital, or see her on a daily basis in the outpatient clinic until the diagnosis is confirmed or rejected.

\section{CONCLUSION}

We could identify only three diagnostic accuracy studies of tests to diagnose rupture of membranes in equivocal cases that fulfilled current methodological criteria. These studies used different testing methods, which made pooling impossible. Based on our findings, 
we conclude that none of the available tests for diagnosing rupture of membranes in women with equivocal rupture of membranes is accurate enough to be used as a single test method. A combination of history, signs and symptoms with clinical insight might be supported with a diagnostic test to decide whether or not the membranes are ruptured. 



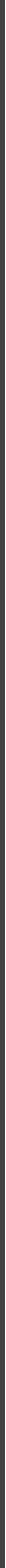




\section{CHAPTER}

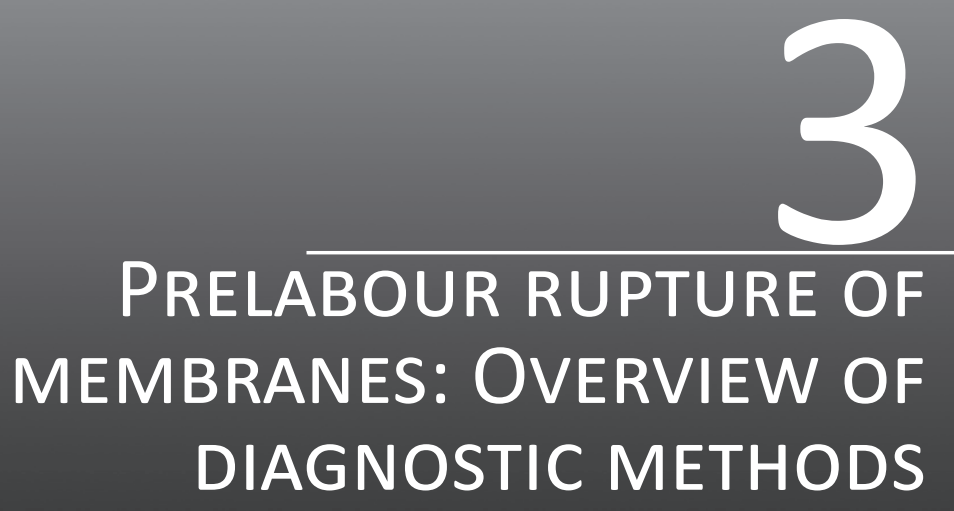

David P van der Ham, Augustinus SP van Teeffelen, Ben W.J Mol

Curr Opin Obstet Gynecol. 2012 Dec;24(6):408-12. 


\section{ABSTRACT}

\section{PURPOSE OF REVIEW}

To evaluate diagnostic accuracy studies for rupture of the fetal membranes (ROM).

\section{RECENT FINDINGS}

Sample sizes of recent studies are small and studies used different 'silver standard' definitions for ROM. Therefore, reported results should be interpreted with caution. Over the review period the focus of diagnostic studies has been on two bedside test strips: insulin-like growth factor-binding protein-1 (IGFBP-1) and placental $\alpha$-microglobulin-1 (PAMG-1). Bedside tests improve the confidence of the clinician about their diagnosis. Compared to nitrazine or ferning test alone, IGFBP-1 and PAMG-1 are more accurate. However, compared to the conventional testing (combination of history, ferning, nitrazine, speculum and ultrasound) no statistical difference in accuracy was found. In-vitro PAMG-1 is shown to be superior to IGFPB-1. Furthermore, soluble intercellular adhesion molecule-1 and AxI receptor tyrosine kinase $(A x I)$ seem to be promising new specific biomarkers for diagnosing ROM.

\section{SUMMARY}

IGFBP-1 and PAMG-1 are the most commonly used bedside test for diagnosing ROM. Both tests seem to be sensitive and specific, however, evidence is lacking especially in equivocal cases and comparative studies against the real golden standard (amnio-dye) have still not been published. Further effectiveness research is needed before tests can be applied in practice. 


\section{INTRODUCTION}

Prelabour rupture of the fetal membranes (PROM) complicates $5-10 \%$ of all pregnan$\operatorname{cies}^{28,29}$ and it is associated with an increased incidence of chorioamnionitis, prematurity and with increased perinatal and maternal morbidity and mortality. ${ }^{1}$ In the majority of women, the diagnosis of ruptured fetal membranes can be based on a history of PROM with speculum examination. This clinical approach has, however, a $12 \%$ false-negative rate. ${ }^{71}$ In approximately $10 \%$ of all cases, the diagnosis of rupture of membranes is difficult to establish. ${ }^{2,10}$

In order to improve the accuracy to diagnose PROM a wide variety of tests have been introduced, the first one to be alkaline testing introduced in the $1930 \mathrm{~s}^{32}$ For decades, a combination of visual pooling of amniotic fluid during speculum examination, alkaline $\mathrm{pH}$ determination and microscopic evidence of ferning and decreased amniotic fluid by ultrasound has been widely used to determine rupture of membranes. This combination has been referred to as 'conventional testing'. These tests, however, are prone to falsepositive results secondary to vaginal contamination with blood, urine, or semen. ${ }^{10-13}$ Besides the inaccuracy of the conventional test, many women find the use of a speculum examination intrusive. ${ }^{14}$ In order to improve the accuracy of diagnostic test and simplify test procedures without the use of a speculum, dozens of tests have been developed over the last decades. In this review, we highlight and report the diagnostic accuracy studies on diagnostic test for PROM that have been published since a systematic review on diagnostic methods for ROMs in equivocal cases. ${ }^{72}$

\section{SYSTEMATIC REVIEW}

We performed a systematic review to assess the accuracy of several tests for the diagnosis of ROM in equivocal cases. ${ }^{72}$ Over a review period from 1960 to September 2010 we identified and obtained 146 full manuscripts, 133 were excluded due to multiple reasons. The remaining 13 studies were scored by an expert panel. Only three studies ${ }^{45,58,70}$ with a total of 155 patients fulfilled all criteria for a diagnostic test accuracy studies. ${ }^{61-64}$ These articles tested three different methods, $\mathrm{pH}$ measurement (64 patients), ${ }^{58}$ insulin-like growth factor binding protein-1 (IGFBP-1, 83 patients) ${ }^{45}$ and a fetoprotein (AFP, eight patients). ${ }^{70}$ Sensitivity varied from $88 \%(\mathrm{pH})$ to $100 \%$ (AFP), specificity varied from $56 \%$ (IGFBP-1) to $100 \%$ (AFP). Based on the limited evidence on the accuracy of tests to diagnose ruptured membranes, we concluded that the use of a particular test cannot be recommended. ${ }^{72}$ For the present review, we repeated our search strategy for the period September 2010 until May 2012 and found seven new articles, which will be described in more detail. 


\section{THE LACK OF A GOLD STANDARD}

Amniocentesis with infusion of a dye is widely considered as the gold standard for the diagnosis of rupture of membranes. However, this procedure is invasive, costly and may itself cause rupture of membranes and other complications, such as infections. ${ }^{57-60}$ Because of this, many researchers and medical ethical committees find it unethical to expose women to amnio-dye infusion. However, over the last decades due to better ultrasound technology, success rate of amniocentesis improved and complication rate seems nowadays to be low. ${ }^{73}$

Studies reporting adverse outcome were published between 1976 and 1983.57,59,60 The last published study, which used amnion infusion with a dye as a gold standard was performed more than 15 years ago. In that study, it took researchers 12 year to include 64 women. ${ }^{70}$ Recently, preliminary results have been published in which placental $\alpha$-microglobulin 1 (PAMG-1) was compared to amnio-dye test. ${ }^{74}$ The final results have, however, not been published yet.

Meanwhile, the lack of a noninvasive gold standard test for PROM is a severe limitation to study (new) diagnostic tests. ${ }^{75}$ The recently published studies that are discussed in this review all lack the use of a gold standard and still do not meet all the criteria for the methodological assessment and reporting of diagnostic accuracy studies as suggested in previously reported guidelines. ${ }^{61-64}$

\section{RATIONALE OF CURRENT DIAGNOSTIC TESTS}

Because of a lack of a gold standard for the majority of the clinical studies and the limitations and inaccuracy of 'conventional testing' as well as the need for a less invasive, less intrusive method, researches have been searching for the identification biochemical markers which are present in high quantities in case of ROM, and absent in cervicovaginal discharge when membranes are intact. Many of these markers have shown to be less valuable because they are also present in other physiological fluid such as blood, vaginal secretion of seminal fluid. ${ }^{75}$ Other markers such as fetal fibronectin seem to indicate the mechanical or inflammatory-mediated detachment of the membranes from the decidua and are nowadays merely used as a predictor for preterm delivery and are no longer considered to indicate ruptured membrane. ${ }^{42,43,76}$

Insulin-like growth factor binding protein-1 (IGFBP-1) and PAMG-1 meet the criteria of high concentration in amniotic fluid and low concentration in other physiological fluids. ${ }^{53,77}$ Therefore, in the past year the focus of the research has been on these two tests. ${ }^{78-83}$ Other proteins, such as soluble intercellular adhesion molecule-1 (sICAM-1) and 
AxI receptor thyrosine kinase $(\mathrm{Axl})$ might be used as biomarkers in the future. ${ }^{84}$

\section{A BEDSIDE TEST TO IMPROVE A DOCTOR'S CONFIDENCE}

Neil and Wallace studied the clinical utility of PAMG-1 testing in daily practice. ${ }^{81}$ They questioned how often and in whom a bedside test might enhance the clinical diagnosis of PROM and change clinical management. In a prospective observational study they included 184 women (100 term pregnancies and 84 preterm pregnancies) in a 12-month period. Based on history and clinical examination (speculum examination) the attending obstetrician was certain with the diagnosis in 53\% of the women and uncertain in $47 \%$. Obstetricians were more confident with preterm women than with term women ( $P=0.02)$. The confidence in the initial diagnosis (ROM or intact membranes) increased significantly when the obstetrician knew the result of the PAMG-1 test. Post test result the obstetrician was certain in $92 \%$ of the women with his/her diagnosis $(P<0.0001)$.

Diagnosis and management was changed after PAMG-1 test especially in the proposed intact membranes group (toward proposed ruptured membranes, 14 out of 82 cases, 17\%). The study did not test the accuracy of the PAMG-1 test, nor did it follow the women until delivery, not giving any insight in the effect on outcome. Results on the accuracy of PAMG1 in this study should, therefore, be interpreted with caution. This study, however, does show the need for clinicians to increase his/her confidence by using a bedside test. ${ }^{81}$

\section{DIAGNOSTIC ACCURACY STUDIES}

Pollet-Villard et al..$^{82}$ studied in vitro the sensitivity of IGFBP-1 and PAMG-1 using different detection limits after dilution of amniotic fluid in a comparative study. They recruited 41 women over 37 weeks of gestational age who were scheduled for a caesarean section. During the caesarean section $0.5 \mathrm{ml}$ samples of amniotic fluid were collected with a syringe before rupture of the membranes and fetal extraction. The samples were diluted with physiological saline solution ( $\mathrm{NaCl} 0.9 \%$ ) in a 1:10, 1:20, 1:40, 1:80, 1:160, 1:320 and 1:640 dilution series. For each dilution both IGFBP-1 and PAMG-1 tests were performed. Up to a dilution of 1 : 40 PAMG-1 showed a sensitivity of 100\%, whereas the sensitivity for IGFBP-1 dropped from 100 to 97.5 to $88 \%$ for $1: 10,1: 20$ and 1:40 dilution, respectively. For the dilution of $1: 40$, this difference was significant $(P<0.05)$. This study tried to mimic the vaginal dilution of amniotic fluid in the vagina after PPROM. However, they only took samples for the term population and it might be questionable whether dilution with $\mathrm{NaCl}$ $0.9 \%$ will actually mimic the clinical condition. Nevertheless, in this in vitro dilution study 
PAMG-1 has a higher sensitivity and better reproducibility than IGFBP-1.82

Two recent articles studied PAMG-1 for the detection of rupture of membranes. ${ }^{78,80}$ The first study was a prospective observational study in 199 women (gestational age 17-42 weeks) with uncertain signs or symptoms of ROM. ${ }^{80}$ Rupture of membranes was first diagnosed using a conventional method with two out of three of the following criteria: positive visual leaking or pooling, positive nitrizine test, amniotic fluid index (AFI) less than $5 \mathrm{~cm}$. PAMG-1 testing was performed after initial diagnosis was made and the investigator was not blinded. Final diagnosis of ROM was made on medical records after delivery. PAMG-1 test was found to be more sensitive (94.4 vs. $72.2 \%, P=0.006$ ) but had the same specificity (98.6 vs. 97.9\%) compared to conventional testing. Due to the costs of ultrasound examination, PAMG-1 testing alone was significantly less expensive than conventional testing. ${ }^{80}$ The second study was an unblinded comparative prospective study in 150 term women (<37 weeks), 75 of whom had definite ROM, based on history (sudden gush), pooling, positive nitrazine and ferning and visual fluid passing the cervical canal during speculum examination, the remaining 75 women had no signs of ROM and were scheduled for induction of labor. ${ }^{78}$ PAMG-1 testing in women with certain ROM had a sensitivity of 97 vs. $84 \%$ for ferning and $87 \%$ for nitrazine test, specificity was 99,79 and $81 \%$, respectively. ${ }^{78}$

Two other studies compared IGFBP-1 and PAMG-1 testing for diagnosis of ROM. ${ }^{79,83}$ In the first prospective observational study 179 women between 16 and 41 weeks of gestation were included. ${ }^{79}$ ROM was primarily diagnosed using a conventional method (pooling, positive ferning, positive nitrazine testing and AFI measurement). The definite diagnosis was made afterwards by two of the researches, unaware of the IGFPB-1 and PAMG-1 test result, based on duration of latency, results of (repeated) speculum examination, (repeated) ferning, nitrazine and decreased AFI by follow-up as well as clinical signs of fetal distress or chorioamnionitis. The presence of at least two of the above was needed for diagnosis of ROM. ${ }^{79}$ The investigators found that the sensitivity $(94,90,87 \%$, for PAMG1, ILGFBP-1 and conventional testing, respectively) and specificity (98, 98 and 95\%, respectively) were high and not statistically different. Related to ferning alone IGFBP-1 and PAMG-1 were significantly more accurate. However, as the researchers commented, ferning or nitrazine testing alone have been shown to be less accurate and are only used in a combined conventional method. ${ }^{79}$ The second study compared ILGBP-1, PAMG-1 and nitrazine testing for diagnosing PROM. In a prospective observational study 100 consecutive women between 17 and 37 weeks of gestation with signs and symptoms of ROM were included. ${ }^{83}$ ROM was diagnosed if three of the following were present: definite pooling, oligohydramion at ultrasound, signs and symptoms of chorioamnionitis, preterm delivery within a week of presentation along with a convincing history of leaking as judged 
by the attending clinician. Medical records were reviewed after delivery. ${ }^{83}$ PAMG-1 had a sensitivity of $93 \%$ and specificity of $100 \%$; IGFPB-1 had a sensitivity of $88 \%$ and specificity of $94 \%$, the difference between both tests was not statistically significant. Compared to nitrazine testing alone, PAMG-1 and IGFPB-1 were significantly more accurate. ${ }^{83}$

In another small observational study vaginal creatine was studied for diagnosing ROM in definite suspected and absent ROM. It was concluded that vaginal creatine might be useful in diagnosing ROM but material and methods of the study were poorly described, therefore, no results are mentioned in this review. ${ }^{85}$

\section{FUTURE TESTING METHODS}

To date, PAMG-1 and IGFBP-1 are the most commonly used bedside test strips for diagnosing ROM. There is, however, some evidence that PAMG-1 might also be a marker for short time to delivery. ${ }^{86}$ Fragmented and phosphorylated forms of IGFBP-1 are associated to predict preterm labor. ${ }^{87,88}$ Like other diagnostic tests in the past, it might be possible that the sensitivity and specificity of PAMG-1 and IGFBP-1 will turn out to be not as high as reported to date. Obviously, researchers are working on new tests, which might be more accurate than currently available ones. ${ }^{84}$ Wang et al. ${ }^{84}$ used a cytokine/chemokinine antibody array in order to identify proteins, which are high in amniotic fluid and low in cervical-vaginal fluid (CVF) and tested these protein in 110 patients with unequivocal ROM and 110 controls. ${ }^{84}$ From the 174 cytokines that were studied in the kit, sICAM1, AxI, IGFBP-1, MCP-1, MIP-1d, TIMP-1 and CD14 were found most interesting. The authors decided to focus on sICAM-1, AxI (AxI receptor tyrosine kinase) and IGFBP-1. sICAM, AxI and IGFBP-1 were respectively 85-fold, 482-fold and 72-fold higher in amniotic fluid than in CVF. sICAM and AxI maybe useful as diagnostics for ROM in which SICAM seems to be a better candidate for the development of a bedside test because the technology to manufacture this test is widely accepted, reliable and inexpensive. ${ }^{84}$

\section{IS THERE A NEED FOR A BEDSIDE TEST?}

There is growing evidence that there is less need for immediate induction of labor when membranes rupture late prematurely. ${ }^{19,89}$ This makes it questionable if for the near-term population a bedside test is needed for a small minority of patients in which the clinician cannot certainly make a diagnosis based on conventional testing. However, in contrast early PPROM is associated with high perinatal morbidity and mortality. Preterm delivery is a frequent sequel of this complication and it is estimated that approximately $25-40 \%$ of preterm deliveries are preceded by PPROM. Although early preterm delivery $(<32$ weeks' 
gestation) occurs in only $1-2 \%$ of total births, it is estimated to account for nearly $50 \%$ of all long-term neurological morbidity and about $60 \%$ of perinatal mortality. ${ }^{90}$ In this perspective, correct diagnosis in equivocal cases is mandatory because a correct diagnosis would bring down unnecessary burden for the health system. False-positive test results could lead to overtreatment (hospital admittance, corticosteroids and antibiotics), whereas a missed diagnosis of PPROM could delay administration of corticosteroids. In equivocal cases, diagnosis of early PPROM is often hindered by vaginal bleeding. In a patient with mild vaginal bleeding in the second trimester the perspective in case of (masked) PPROM is significantly different, and a validated test in this case could be valuable.

\section{CONCLUSIONS}

Since we published a systematic review on the diagnostic tests for rupture of membranes $^{72}$ several new studies have been published. ${ }^{78-85}$ Due to the lack of a noninvasive gold standard, the use of a second best 'silver standard' varies among different studies as well as the included population (preterm, term) and complaints (equivocal or unequivocal ROM). There were no randomized controlled trials; all studies were prospective and observational. Studies had small sample sizes (maximum 199 participants ${ }^{80}$ ) and, therefore, it is difficult to compare different tests with each other. PAMG-1 seems to be the most sensitive and specific test, and is subject of the only study with a design including the real gold standard dye-infusion results still being underway. New tests are being developed. ${ }^{84}$ But as long as current and newly developed tests are not tested against the gold standard, results of new studies will always be issue of debate. Furthermore, in our opinion studies should be more focused on the early PROM group. 


\section{KEY POINTS}

Studies on diagnostic methods for rupture of membranes are small and all lacking a gold standard.

The used second best 'silver standard' varies among studies, which makes results hard to compare and results should be interpreted with caution.

PAMG-1 seems to be superior to IGFBP-1, but new, possibly more accurate tests (sICAM and $\mathrm{AxI}$ ) are being developed.

In order to find the real accuracy of current and newly developed diagnostic methods for ROM, these methods should be tested preferably in large randomized controlled trial(s) against the gold standard (amnio-dye infusion). 


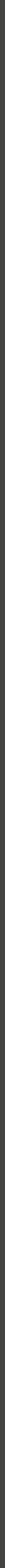




\title{
CHAPTER
}

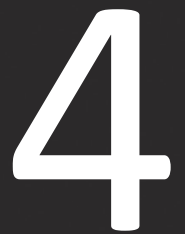

ACCURACY OF C-REACTIVE PROTEIN DETERMINATION IN PREDICTING CHORIOAMNIONITIS AND

NEONATAL INFECTION IN PREGNANT WOMEN WITH PREMATURE RUPTURE OF MEMBRANES:
\end{abstract}

\section{A SYSTEMATIC REVIEW}

Rafli van de Laar, David P van der Ham, S Guid Oei, Christine Willekes,

Carl P Weiner, Ben W.J Mol

Eur J Obstet Gynecol Reprod Biol. 2009 Dec;147(2):124-9. Epub 2009 Oct 12 


\section{SUMMARY}

Preterm premature rupture of the fetal membranes (PPROM) is associated with intra-uterine infection. Early detection of intra-uterine infection may help prevent neonatal sepsis. C-reactive protein (CRP) is an acute phase protein often elevated when inflammation is present. The aim of this review was to assess whether CRP accurately predicts chorioamnionitis and/or neonatal sepsis in women with PPROM.

We searched Medline and Embase databases for articles reporting on CRP and chorioamnionitis and/or neonatal sepsis. Two reviewers extracted clinical and methodological study characteristics and test accuracy data. Accurate data were used to form $2 \times 2$ data tables comparing CRP and the occurrence of infection. For the selected studies, sensitivity and specificity of CRP in the prediction of histological chorioamnionitis, clinical chorioamnionitis and neonatal sepsis were calculated separately. A bivariate meta-regression model was used to calculate pooled estimates of sensitivity and specificity.

The search revealed 200 articles, of which only five met the inclusion criteria. These five articles reported on 381 patients, of which four articles (227 patients) reported on CRP as a predictor for histological chorioamnionitis and four studies (330 patients) reported on CRP as a predictor for clinical chorioamnionitis. None of the selected articles fulfilled our criteria for the use of CRP as a predictor of neonatal sepsis. CRP was moderately predictive of histological chorioamnionitis. Unfortunately, the studies of clinical chorioamnionitis were too heterogeneous to pool data.

Current literature does not support the use of CRP in women with PPROM. 


\section{INTRODUCTION}

Preterm premature rupture of the fetal membranes (PPROM) affects 2 to $4.5 \%$ of all pregnancies and is associated with maternal and perinatal morbidity and mortality. ${ }^{1,32,91}$ One of the main causes of perinatal morbidity and mortality is intrauterine infection. Thus, the prediction of infection in patients with PPROM may facilitate timely management to avert morbidity and mortality in the mother but especially in the neonate. C-reactive protein (CRP) is widely used in clinical practice, particularly for the early diagnosis of chorioamnionitis in patients with PPROM. CRP is an acute phase protein synthesized in the liver during infection. Several studies have previously reported a positive correlation between elevated maternal serum CRP levels and clinical and/or histological chorioamnionitis and neonatal morbidity and mortality. ${ }^{92-96}$ However, these studies vary widely in their definition of chorioamnionitis, maternal and fetal outcomes and optimal cut-off levels of CRP in predicting maternal and fetal complications, thus hampering a fundamental decision as to whether CRP should be routinely measured in women with PPROM. Furthermore, most studies are of poor methodological quality and will not pass current standards. ${ }^{64}$

Recently, Trochez-Martinez et al. published a systematic review on the use of CRP as a predictor for histological chorioamnionitis in patients with PPROM. ${ }^{97}$ This review included an article in which the authors included patients with preterm PROM as well as term PROM without extracting only the preterm data, ${ }^{98}$ a study suffering a partial verification bias by performing a histological examination only in women with proven clinical chorioamnionitis, ${ }^{99}$ a study with a selection bias incurred by only examining the placental histology of women delivered within $72 \mathrm{~h}$ after amniocentesis for microbiologic assessment of the amniotic cavity after PPROM, ${ }^{100}$ and a study in which the reference test on histological chorioamnionitis was not clearly reported. ${ }^{101}$

Furthermore they included a study by Nowak et al. ${ }^{102}$ in which a selection bias was created by only including primiparous patients. Most importantly, the authors did not search for studies on neonatal sepsis.

In our opinion it is more relevant to predict and prevent neonatal sepsis and clinical chorioamnionitis rather than histological chorioamnionitis without clinical signs of infection in mother and newborn. This is the reason why we conducted this systematic review on use of CRP in prediction of histological and clinical chorioamnionitis as well as neonatal sepsis. 


\section{MATERIALS AND METHODS}

\section{SEARCH STRATEGY}

We conducted an electronic search in Medline (1951-2007) and Embase (1974-2007) databases using MeSH terms C-reactive protein; chorioamnionitis; fetal membrane, premature rupture; infant, newborn; sepsis and pregnancy complication, infectious, using Boolean operator 'OR' and 'AND' as appropriate, without language restrictions. Selected papers in languages other than English were translated by medically qualified professionals. We checked reference lists of known reviews and primary articles to identify cited articles not captured by electronic searches.

All studies evaluating the use of CRP in pregnant women with rupture of fetal membranes before 37 completed weeks of gestational age were selected in a two-stage process. First, two independent reviewers (RL, DH) carried out the electronic search. Both scrutinized all titles and/or abstracts for eligible studies. Second, full manuscripts were obtained from selected articles based on their abstract and from studies that were lacking abstracts. Reference lists of all primary articles were examined to identify cited articles not found in the initial electronic search. Any disagreement was resolved by consensus or if necessary by a third reviewer (BM).

\section{SELECTION CRITERIA}

Studies were included in which pregnant women with rupture of membranes before 36 completed weeks of gestational age (PPROM) had maternal serum CRP (index test) tested. The outcome measures were neonatal sepsis defined as a positive blood culture or clinical signs of infection (apnea, fever, intolerance for feeding, respiratory distress and/ or hemodynamic instability) with positive surface cultures; suspicion of clinical chorioamnionitis defined as a temperature of at least $37.5^{\circ} \mathrm{C}$ in combination with at least one of the following: uterine tenderness, purulent amniotic fluid, maternal or fetal tachycardia, ${ }^{103}$ and histological chorioamnionitis defined as the presence of neutrophil infiltrate in extraplacental membranes.

\section{QUALITY ASSESSMENT}

We considered all studies comparing CRP to either chorioamnionitis or neonatal sepsis eligible for our review. An adequate description of reference and index test(s) had to be available. We excluded all case-control design studies and all studies with inappropriate description of the spectrum of patients as these are known to result in statistical bias and spectrum bias, respectively. ${ }^{64,104}$ Data on clinical and methodological study characteristics and on test accuracy were extracted by two independent reviewers (RL, 
Total studies identified from electronic search $(n=200)$

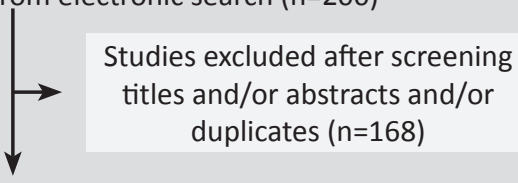

Studies of accuracy of C-reactive protein determination in predicting chorioamnionitis and/or neonatal infection in pregnant women with premature rupture of membranes retrieved for detailed evaluation From electronic search $(n=42)$

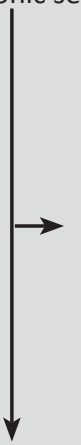

Studies excluded with reason

Inappropriate population $(n=14)$

Data to construct $2 \times 2$ table not extractable $(n=11)$

Not a test accuracy studie $(n=7)$ Lack of original data i.e. review or letters $(n=2)$

Inadequate test description $(n=1)$

Unable to translate $(n=2)$

Primary studies included in systematic review $(n=5)$

Figure 4.1 | Study selection process

$\mathrm{DH})$. The data extracted were placed on data extraction forms using the QUADAS-tool. ${ }^{64}$ Characteristics extracted included: study design, consecutive patient recruitment, prospective data collection, details of study population (inclusion/exclusion criteria, use of antibiotics/tocolysis/steroids), detailed description of the index test (CRP) and reference test, blinding missing data number of patients with index test and reference test. The following items were considered clinically relevant: use of antibiotics, timing of the index test, blinding for index and reference test, incidence and details of outcome.

\section{DATA SYNTHESIS}

We constructed one or more $2 \times 2$ tables for each study, cross classifying CRP test results against the occurrence of clinical and/or histological chorioamnionitis and/or neonatal morbidity/mortality. Where necessary we altered CRP concentrations in milligram per liter (mg/l). We planned to use receiver operating characteristic (ROC) plots to visualize data. We used a bivariate meta-regression model to calculate pooled estimates of sensitivity and specificity for several CRP cut-off values and to fit a summary ROC (sROC) curve. This method has been extensively described elsewhere. ${ }^{105-107}$ Rather than using a single outcome measure per study, like the diagnostic odds ratio (DOR), the bivariate model 


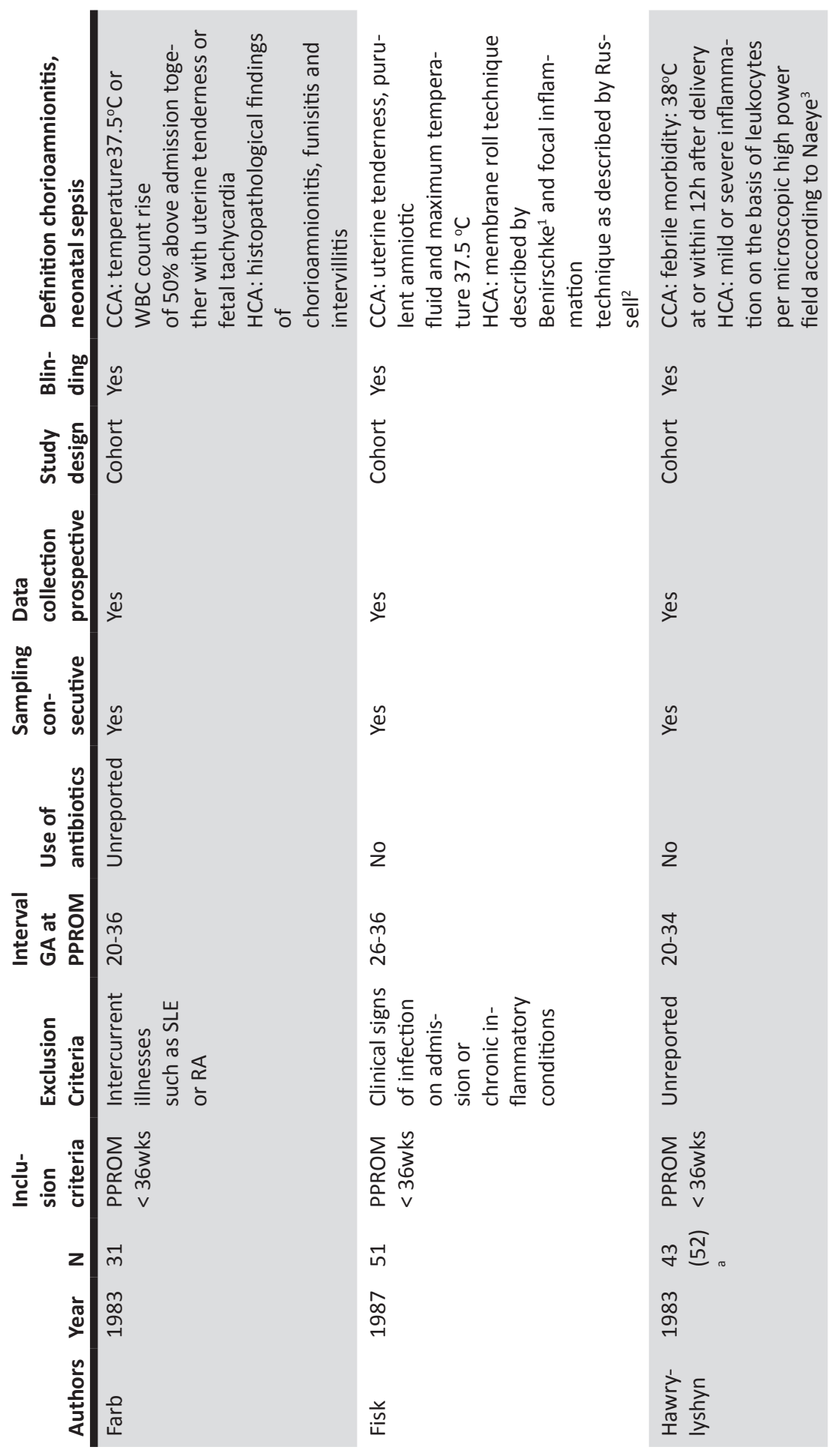



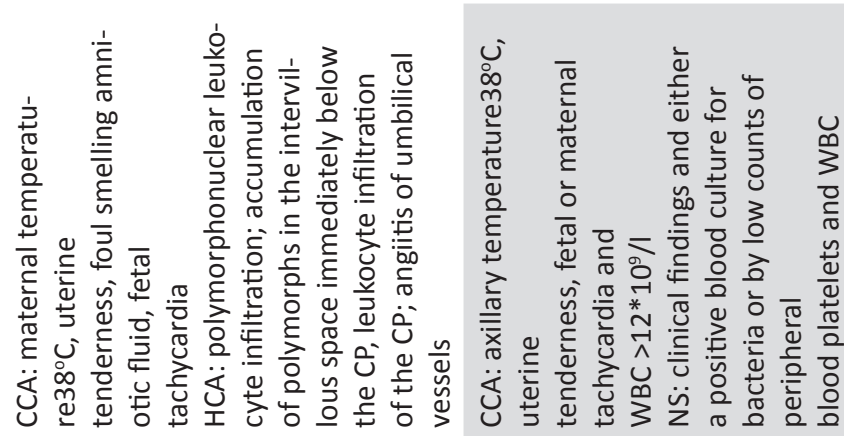

$\stackrel{n}{\succ}$
$\frac{5}{0}$
$\frac{5}{0}$

운
$\frac{1}{0}$
$\frac{1}{0}$

$\stackrel{\coprod}{\check{\nu}}$

$\stackrel{\Perp}{\searrow}$

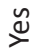

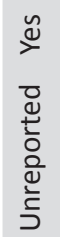

$\stackrel{\stackrel{n}{m}}{\stackrel{\omega}{N}}$

i̊

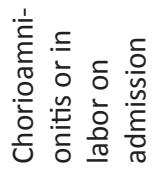

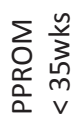

욱

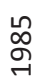

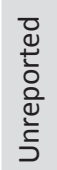

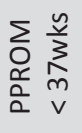

守

욤

产

羊
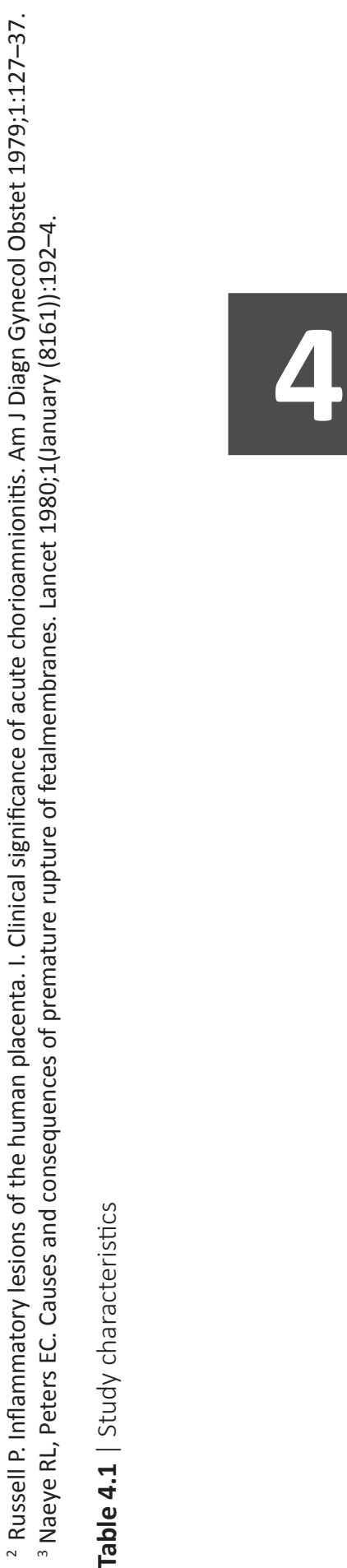
preserves the two-dimensional nature of diagnostic data in a single model. This model incorporates the correlation that may exist between sensitivity and specificity within studies due to possible differences in threshold between studies. The bivariate model uses a random effects approach for both sensitivity and specificity, allowing for heterogeneity beyond chance due to clinical or methodological differences between studies. In addition, the model acknowledges the difference in precision by which sensitivity and specificity have been measured in each study. This means that studies with a larger number of chorioamnionitis patients have more weight in the calculation of the pooled estimate of sensitivity, while studies with fewer infected patients are more influential in the pooling of specificity. We calculated a correlation coefficient ( $r$ ) to show the strength and shape of the correlation between sensitivity and specificity, and hence the amount of variance that can be explained due to threshold differences ( $r 2$ ). Despite several cut-off values for CRP, each study was represented once in the summary ROC analysis. The Proc NLMixed procedure in SAS version 9.1 for Windows (SAS Institute Inc., Cary, NC, USA) was used to fit the bivariate models.

\section{RESULTS}

Figure 4.1 summarizes the process of study identification and selection. Five primary articles met the selection criteria. Four articles had histological chorioamnionitis as (one of their) endpoint(s); four articles had clinical chorioamnionitis as (one of the) endpoint(s). None of the selected primary articles fulfilled our criteria for neonatal sepsis. Table 4.1 shows the methodological and reporting characteristics of the included studies. We were unable to translate two articles, ${ }^{108,109}$ due to absence of a qualified medical translator. Table 4.1 summarizes the study characteristics of the included studies.

Table 4.2 shows the results of CRP in histological chorioamnionitis and clinical chorioamnionitis. Gestational age ranged between 20 and $36^{+6}$ weeks; mean and median gestational ages among the studies could not be calculated. The interval between the last CRP determination and delivery varied between 12 and 72h; the majority of the studies had CRP testing at least every $24 \mathrm{~h}$. The reported prevalence of histological chorioamnionitis among the included studies was $54.6 \%$ (124/227; range: $21-63 \%)$, the prevalence of clinical chorioamnionitis was $25.8 \%$ (85/330; range: $18-50 \%)$.

A plot of sensitivity-specificity points and an SROC curve illustrating the predictive capacities of CRP in women with PPROM for histological chorioamnionitis and for clinical chorioamnionitis are illustrated in figure 4.2 and figure 4.3, respectively. The sROC curve is not extended towards the ends of the graph (figure 4.2) because we lacked reliable information of sensitivity and specificity in the extreme ranges. We were therefore unable 
Accuracy of CRP in predicting chorioamnionitis and sepsis

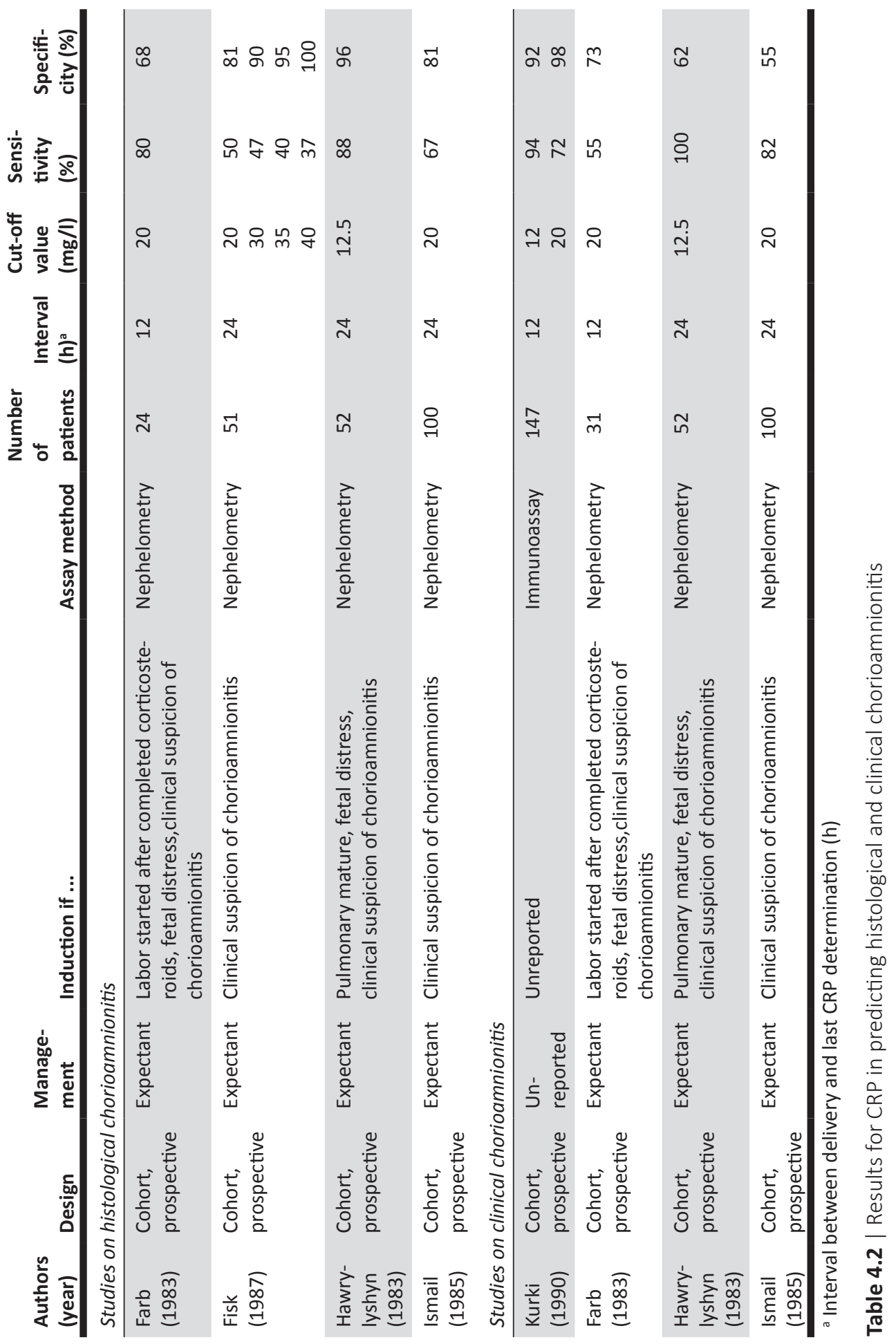


to calculate an area under the curve. We found significant heterogeneity among studies reporting on clinical chorioamnionitis (figure 4.3). As a consequence, a reliable sROC curve could not be estimated for clinical chorioamnionitis.

\section{Discussion}

For this review, we identified five studies that reported on 381 patients with CRP measurements and PPROM. Of these, four (227 patients) considered the potential of CRP to be a predictor of histological chorioamnionitis and four (330 patients) a predictor of clinical chorioamnionitis. None of the selected articles fulfilled our predetermined criteria for CRP as a predictor for neonatal sepsis.

Trochez-Martinez et al. conducted a systematic review on CRP as a predictor of chorio-

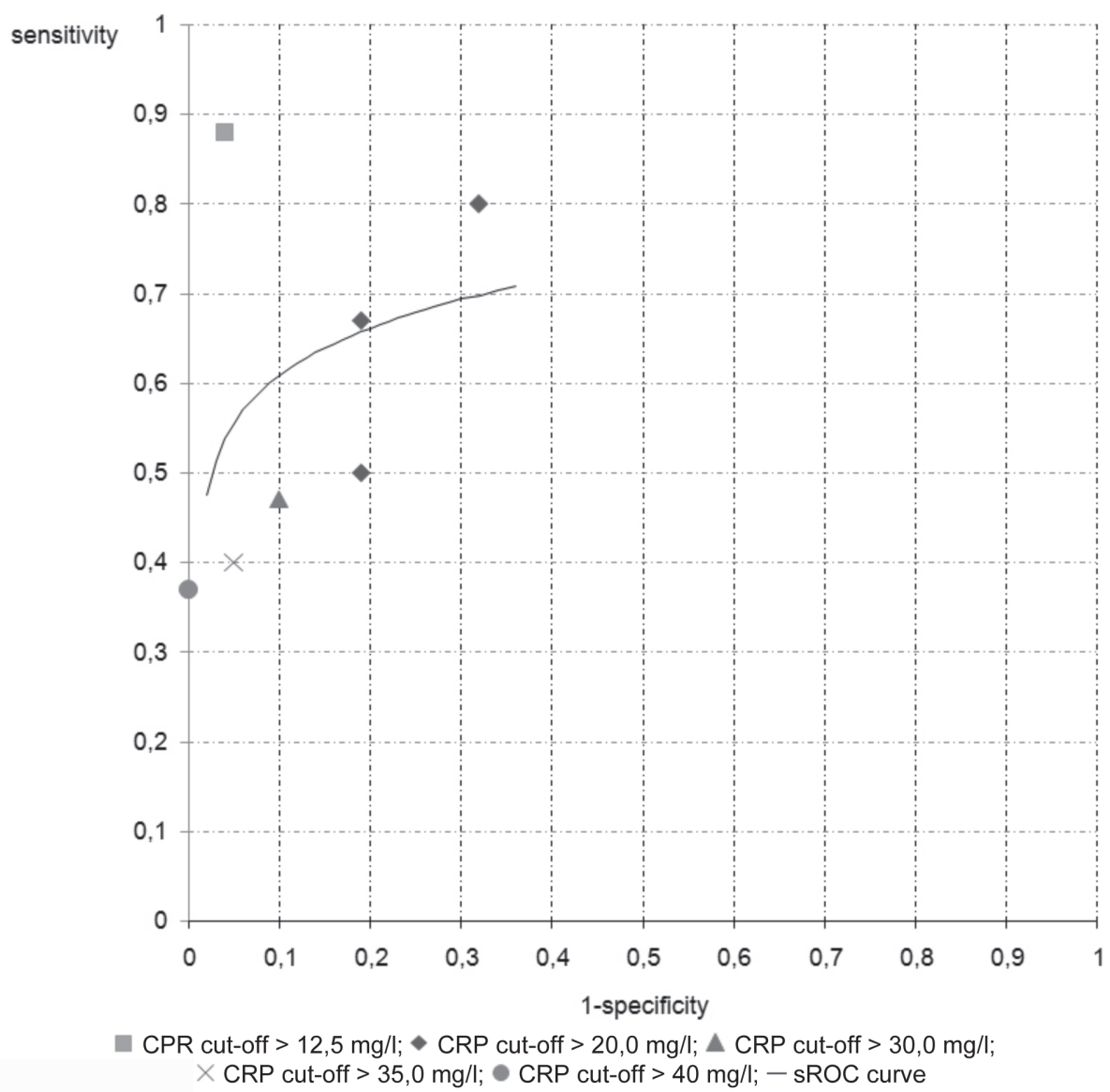

Figure 4.2 | sROC curve for CRP and histological chorioamnionitis 
amnionitis in women with PPROM. ${ }^{97}$ They used histological chorioamnionitis as the gold standard, noting that clinical chorioamnionitis was easier to diagnose and that further testing (e.g., CRP) might delay the management of these patients. In our opinion, however, it is more relevant to predict and prevent clinical chorioamnionitis and neonatal sepsis rather than histological chorioamnionitis without clinical signs of infection. It should be kept in mind that in a minority of cases nonbacterial chorioamnionitis occurs. In these cases, CRP is not indicative of disease and can give a false negative result. ${ }^{110}$ In contrast to Trochez-Martinez et al. we included four instead of eight studies on CRP determination and histological chorioamnionitis. We excluded four studies due to an inappropriate spectrum of patients, ${ }^{98}$ an inadequate or inappropriate description of the reference test, ${ }^{101}{ }^{\text {se- }}$ lection bias ${ }^{100,102}$ or partial verification bias. ${ }^{102}$ Trochez-Martinez et al. excluded the study by Ismail et al. because they were unable to extract the raw data. Using sensitivity and

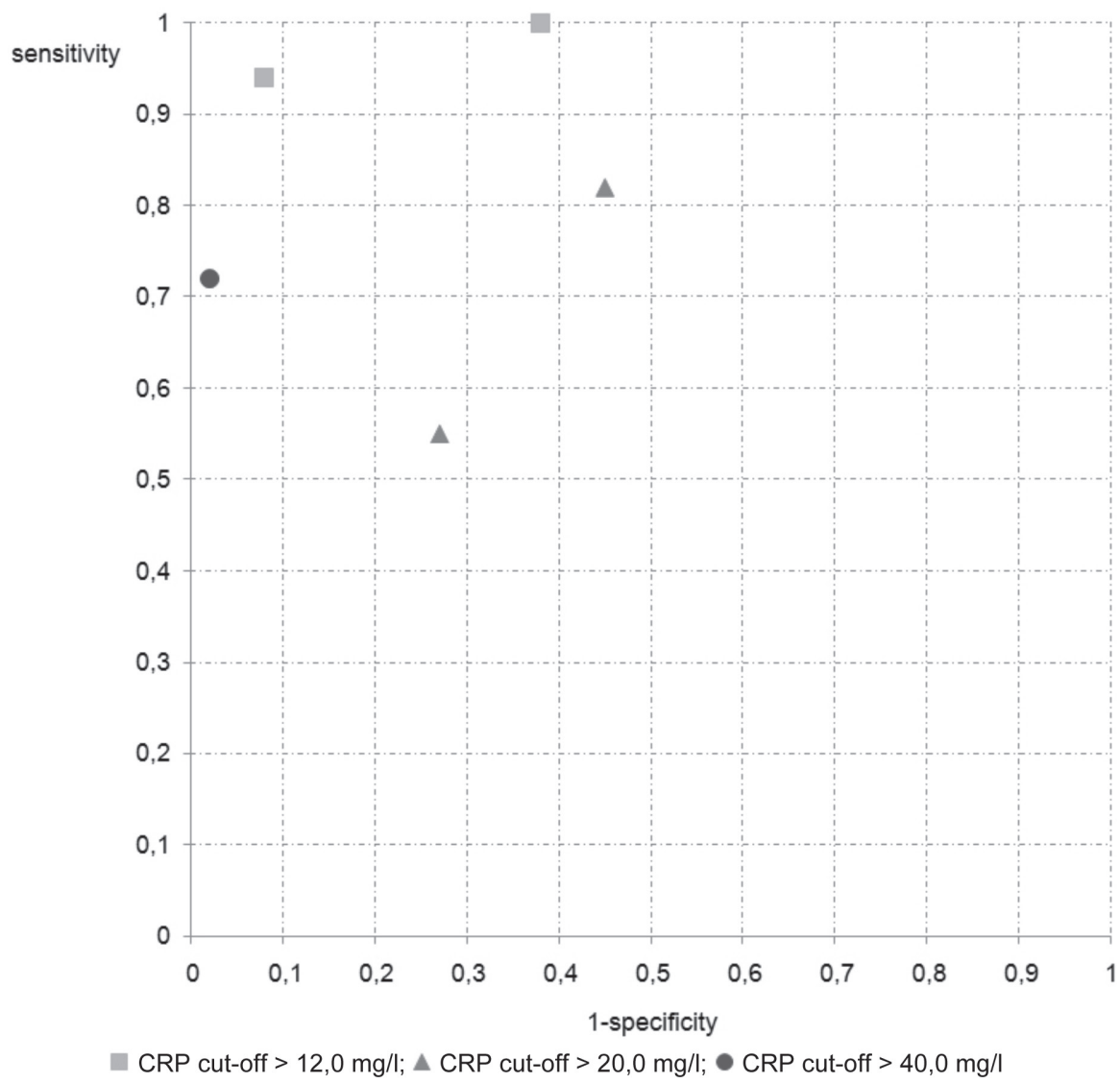

Figure 4.3 | sROC curve for CRP and clinical chorioamnionitis 
specificity combined with the total number of included patients we were, in contrast, able to extract the raw data. ${ }^{111}$ Despite disagreement in methods, selection and use of reference test, we agree with Trochez-Martinez et al. ${ }^{97}$ that there is no clear evidence to support the use of CRP as an accurate diagnostic test of histological chorioamnionitis. The quality from the included studies was poor. All included studies were prospective consecutive cohort studies. The lack of blinding ${ }^{99}$ and unreported exclusion criteria93,99,112 might have influenced the study results.

Furthermore, the wide interval in gestational age at inclusion in some studies, varying from 20 to 36 weeks, combined with a small number of patients may have overestimated the accuracy of CRP, especially in predicting histological chorioamnionitis since in the very premature pregnancies, one might manage a patient expectantly after the first signs of infection. A CRP rise in these patients might therefore seem to correctly diagnose histological chorioamnionitis. However, whether histological chorioamnionitis was present before or at the moment the CRP rise occurred, will always be uncertain. The influence of the use of antibiotics is not clear; in two studies they did not use antibiotics ${ }^{93,113}$ and in the other three studies they did not report the use of antibiotics..$^{99,111,114}$

The prevalence of histological and clinical chorioamnionitis among the studies is high (54.6 and 25.8\%). Ramsey et al. in a retrospective cohort of 430 patients with PPROM found a prevalence of $13 \%$ for clinical chorioamnionitis (gestational age 24-37 weeks [mean 32.4 weeks]). ${ }^{115}$ In another study of preterm births, the prevalence of histological chorioamnionitis ranged between 26 and 30\%. ${ }^{116}$ Difference between prevalence of the included studies and prevalence described by others may probably overestimate the incidence of chorioamnionitis among the included studies and therefore lead to a better accuracy of CRP determination. We doubt the clinical usefulness of CRP even if it is an accurate predictor for histological chorioamnionitis because in predicting histological chorioamnionitis, one does not know whether the patient will develop clinical symptoms, or more importantly, whether the neonate will be infected. The only thing we know is that the chance a mother or her baby will be infected is increased. When at or near term, the decision to induce labor might be justifiable. However, incorrectly inducing labor in a preterm or very preterm pregnancy may lead to serious complications for the neonate. As a result, the prediction of clinical chorioamnionitis, or even better neonatal sepsis, might be more valuable. Unfortunately, we were unable to identify a single study on CRP and neonatal sepsis. Further, the studies on clinical chorioamnionitis were very heterogeneous using a wide variety of sensitivity and specificity of CRP for various cut-off levels. Therefore, we were unable to construct a reliable sROC curve. These results cannot support nor oppose the accuracy of CRP determination in predicting clinical chorioamnionitis. 


\section{CONCLUSION}

Based on existing literature, we are unable to support the use of CRP as a predictor for chorioamnionitis or neonatal sepsis following PPROM. However, we are also unable to conclude that CRP is ineffective in detecting chorioamnionitis or predicting neonatal infection. We cannot recommend the use of CRP in the management of women with PPROM as the only predictor for clinical chorioamnionitis. Further research, preferably in a randomized controlled blinded setting with larger populations, should be conducted to determine the usefulness of CRP in its prediction of clinical chorioamnionitis and more importantly neonatal sepsis in women with PPROM. 


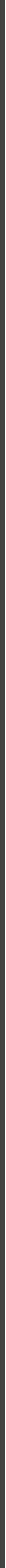




\section{CHAPTER}

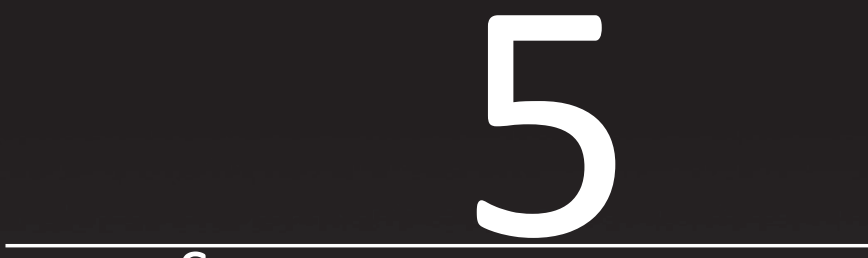

STUDY PROTOCOL

INDUCTION OF LABOUR VERSUS EXPECTANT MANAGEMENT IN WOMEN WITH PRETERM PRELABOUR RUPTURE OF MEMBRANES BETWEEN 34 AND 37

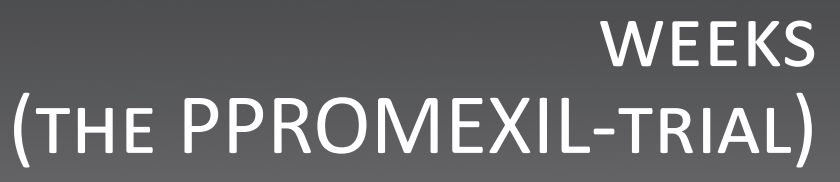

David P van der Ham, Jan G Nijhuis, Ben Willem J Mol, Johannes J van Beek, Brent C Opmeer, Denise Bijlenga, Mariette Groenewout, Birgit Arabin, Kitty WM Bloemenkamp, Wim J van Wijngaarden, Maurice GAJ Wouters,

Paula JM Pernet, Martina M Porath, Jan FM Molkenboer, Jan B Derks, Michael M Kars, Hubertina CJ Scheepers, Martin JN Weinans, Mallory D Woiski, Hajo IJ Wildschut and Christine Willekes BMC Pregnancy Childbirth. 2007 Jul 6;7:11. 


\section{ABSTRACT}

\section{BACKGROUND}

Preterm prelabour rupture of the membranes (PPROM) is an important clinical problem and a dilemma for the gynaecologist. On the one hand, awaiting spontaneous labour increases the probability of infectious disease for both mother and child, whereas on the other hand induction of labour leads to preterm birth with an increase in neonatal morbidity (e.g., respiratory distress syndrome (RDS)) and a possible rise in the number of instrumental deliveries.

\section{METHODS/DESIGN}

We aim to determine the effectiveness and cost-effectiveness of immediate delivery after PPROM in near term gestation compared to expectant management. Pregnant women with preterm prelabour rupture of the membranes at a gestational age from $34^{+0}$ weeks until $37^{+0}$ weeks will be included in a multicentre prospective randomised controlled trial. We will compare early delivery with expectant monitoring. The primary outcome of this study is neonatal sepsis. Secondary outcome measures are maternal morbidity (chorioamnionitis, puerperal sepsis) and neonatal disease, instrumental delivery rate, maternal quality of life, maternal preferences and costs. We anticipate that a reduction of neonatal infection from $7.5 \%$ to $2.5 \%$ after induction will outweigh an increase in RDS and additional costs due to admission of the child due to prematurity. Under these assumptions, we aim to randomly allocate 520 women to two groups of 260 women each. Analysis will be by intention to treat. Additionally a cost-effectiveness analysis will be performed to evaluate if the cost related to early delivery will outweigh those of expectant management. Long term outcomes will be evaluated using modelling.

\section{DISCUSSION}

This trial will provide evidence as to whether induction of labour after preterm prelabour rupture of membranes is an effective and cost-effective strategy to reduce the risk of neonatal sepsis.

\section{Controlled clinical trial register: ISRCTN29313500}




\section{BACKGROUND}

Preterm prelabour rupture of the membranes (PPROM) is an important clinical problem and a dilemma for the gynaecologist. On the one hand, awaiting spontaneous labour may lead to an increase in infectious disease for both mother and child, whereas on the other hand induction of labour leads to preterm birth with an increase in neonatal morbidity (e.g., respiratory distress syndrome (RDS)) and a possible rise in the number of instrumental deliveries.

The estimated incidence of PPROM between 34 and 37 weeks of gestation is 1.5\%, which equals about 3.000 cases per year in the Netherlands. The incidence of RDS is estimated to decrease from $15 \%$ at 34 weeks to below $1 \%$ at 37 weeks' gestation. ${ }^{117}$ On the other hand, the probability that sepsis occurs increases when expectant management is advocated. In case the child is born immediately after PPROM, the risk of sepsis is $2.5 \%$, whereas it increases to $7.5 \%$ in case of expectant management. ${ }^{103,118}$

Until now, management of PPROM between 34 and 37 weeks' gestation varies in the Netherlands. In the guideline of the Dutch Society of Obstetrics and Gynaecology (NVOG) expectant management is advocated if the gestational age is under 35 weeks. ${ }^{5}$ Beyond 35 weeks, the guideline makes no clear recommendation, and the decision for either induction of labour or expectant management (either in hospital or with monitoring at home), is left to local protocols. International guidelines do not make a clear statement either. ${ }^{15,119}$ This dilemma can be explained by a lack of good clinical evidence. As a consequence, the course of action on this subject varies in the Netherlands. Data from the Dutch National Delivery Registration indicate that in about $70 \%$ of the patients an expectant management has been practised, whereas in about 30\% of the cases labour was induced preterm. The current national registrations provide no insight in the reason for induction in the latter group, as some inductions might have been performed following signs of intra-uterine infection. In view of the lack of good clinical evidence and the practice variation described above, a randomised clinical trial comparing induction of labour and expectant monitoring in patients with PPROM is needed urgently.

We are aware of four small studies that have been performed to discern between the treatment policies for PPROM. 20,23,24,26 Naef et al. compared induction of labour versus expectant management in a group of 120 patients with PPROM occurring at 34 until 37 weeks gestation and found a nonsignificant decrease of chorioamnionitis in the induction group without a significant difference in neonatal morbidity (including RDS) between both groups. ${ }^{24}$ Patients with PPROM managed expectantly were hospitalised significantly longer. More babies of the expectant group were diagnosed with sepsis and admitted to the neonatal ward for a longer duration. This difference did not reach the level of signifi- 
cance due to the small number of the study. Cox et al. compared maternal and neonatal outcome in a group of 129 patients with PPROM occurring at 30 until 34 weeks' gestation after randomisation between expectant management and induction of labour. ${ }^{20}$ Again, no significant differences in neonatal outcome were noted. However, a nonsignificant decrease in sepsis was seen in the induction group. Also, chorioamnionitis was observed less frequently in the latter group. Mercer et al. performed a similar study of 93 patients with PPROM in a different age category (32 until 36 weeks' gestation) and observed similar differences as Cox and co-workers. ${ }^{23}$ Spinnato et al. found no difference in neonatal outcome between expected management and prompt delivery in 47 patients with premature rupture of membranes (before 36 weeks) with documented foetal pulmonary maturity. ${ }^{26}$ However, they demonstrated an increased risk of maternal infection when expectant management was applied. These studies show a trend towards better neonatal outcome in the induction group, but in these small samples differences were not statistically significant. Even meta-analysis did not generate sufficient statistical power. ${ }^{120}$ In view of the practice variation described above and the lack of sound clinical evidence for this clinical dilemma, we have recently started a multicentre trial.

\title{
Methods/Design
}

\begin{abstract}
AIMS
The aim of this study is to systematically compare early initiation of delivery and expectant management in case of preterm prelabour rupture of membranes in terms of neonatal sepsis and RDS, maternal health, health-related quality-of-life and costs. As it is impossible to blind the healthcare workers and patients involved for the strategy of allocation, we will use a multicentre randomised controlled open label trial to assess the effects of initiation of delivery or expectant management on neonatal outcome. This study is set in the Dutch Obstetric Consortium, a collaboration of obstetric clinics in the Netherlands. Approximately 40 clinics, including academic hospitals, nonacademic teaching hospitals and nonteaching hospitals will participate in this trial.
\end{abstract}

\section{PARTICIPANTS/ELIGIBILITY CRITERIA}

Women presenting with preterm prelabour rupture of the foetal membranes between $34^{+0}$ and $37^{+0}$ weeks' gestation who have not delivered within 24 hours after rupture of the foetal membranes are eligible for participation in the PPROMEXIL-trial. Even so women presenting with preterm prelabour rupture of foetal membranes after $26^{+0}$ weeks gestation who have not delivered at $34^{+0}$ weeks of gestation are eligible for participation. Both women with singleton and women with multiple gestations can be included. 
Women with a child in breech presentation can also be included, and both an elective caesarean section and a vaginal delivery are allowed for these women. Women with monochorionic multiple pregnancies, abnormal (non-reassuring) cardiotocogram (CTG), meconium stained amniotic fluid, signs of intrauterine infection, major foetal anomalies, labour, HELLP syndrome or severe pre-eclampsia, will not be included in the study.

\section{PROCEDURES, RECRUITMENT, RANDOMISATION AND COLLECTION OF BASELINE DATA}

The research nurse and/or the staff of participating hospitals will identify eligible women. After the subject has given informed consent for participation in the study, she will be randomised to either a policy that aims at termination of the pregnancy (intervention group) or a policy that aims at expectant management for spontaneous delivery (expectant group). Once the subject data have been entered anonymously in a web-based database, either a staff member or the research nurse will randomise using an internet based procedure. Randomisation will be 1:1 for intervention or expectant management and stratified for centre and previous delivery. At study entry baseline demographics, past obstetric and medical history will be recorded. Maternal temperature is measured and baseline blood samples are taken. From all participating women a vaginal swab is collected either at admission to the hospital with premature ruptured membranes or at study entry. Antibiotics are given in accordance to local policy. All women will undergo an ultrasound examination at study entry. If a previous ultrasound examination was done within a fortnight before study entry in the same hospital, which revealed no abnormal results, these outcomes may be recorded at study entry. Women fill out a baseline quality of life questionnaire containing EuroQoL, 5D3L, HADS, SF-36 and background questions. They are also asked on their prior intervention preference. At local centres data-collection will be the responsibility of the local research coordinator and the regional research nurses. The data for this study must be collected, coded and processed with adequate precautions to ensure patient confidentially.

\section{INTERVENTIONS}

\section{Intervention group}

Women randomised for intervention will be planned for induction of labour or elective caesarean section as soon as reasonable after randomisation. Because of the fact that randomisation will take place preferable 24 hours after rupture of the foetal membranes it is imaginable that due to logistic reasons induction of labour is postponed until the following morning, but within 12 hours after randomisation. Induction of labour will take place according to local policy. In case of breech presentation, a primary caesarean 
section is allowed based on the preference of both the patient and the gynaecologist. Similarly in case of a dichorionic twin an elective caesarean section may be performed because of the position of the presentation of the first twin.

\section{Expectant group}

Women randomised for expectant management will be treated according to local policy. This might be either in an outpatient or inpatient setting. If a patient in the expectant group reaches $37^{+0}$ weeks of gestation age, induction of labour is performed according to local policy. Whenever a patient with an indication for elective caesarean section will be allocated to expectant management the caesarean section will be performed as soon as labour commences.

\section{FOLLOW UP OF WOMEN AND INFANTS}

Information is obtained on the condition, weight, length and neonatal morbidity and mortality from the infant and maternal records as well as maternal complications and length of stay. If applicable, neonatal antibiotic treatment, blood samples and cultures are recorded. In case of admittance of the baby to the neonatal intensive care, high care or medium care unit, details of this admittance are also documented. The placenta will be sent for histological examination on chorioamnionitis and funisitis. Six weeks and six months post partum neonatal length, weight, neurological disabilities, physical disabilities and maternal disabilities will be recorded. Patients will be asked to fill out follow-up quality of life questionnaires including EuroQoL, 5D3L, HADS, SF-36, SCL-90 also concerning the development of the child. Women are also asked to (re)state their intervention preference. Long-term follow up of the children is not yet planned, and depends on future funding.

\section{OUTCOME MEASURES}

The primary outcome measure is neonatal sepsis. Neonatal sepsis is defined as a positive blood culture, biochemical infection parameters (C-reactive protein (CRP) above $20 \mathrm{mg} / \mathrm{l}$ ) or clinical signs of infection (apnoea, fever, intolerance for feeding, respiratory distress and/or haemodynamic instability) with positive surface cultures. An independent panel of paediatricians will define between proven or probable sepsis without knowledge of the outcome data.

Secondary infant outcome measures are respiratory distress syndrome (RDS) (defined according to the Organ dysfunction criteria), transient tachypnoea of the newborn asphyxia (defined according to Sarnat), pneumothorax/pneumomediastinum, late onset sepsis, hypoglycaemia, meconium aspiration syndrome, necrotizing enterocolitis (NEC) (defined according to Bell staging), hyperbilirubinaemia, intraventricular haemorrhage, 
periventricular leucomalacia, convulsions, other neurological abnormalities and congenital abnormalities. ${ }^{121-123}$

Secondary maternal outcome measures are ante partum haemorrhage, umbilical cord prolapse, signs of chorioamnionitis (defined as fever before or during labour as a temperature greater than $37,5^{\circ} \mathrm{C}$ on two occasion more than one hour apart or a temperature $>38,0^{\circ} \mathrm{C}$ with either uterine tenderness (or contractions), leucocytosis, maternal or foetal tachycardia, or a foul-smelling vaginal discharge in absence of any other cause of hyperpyrexia), maternal sepsis (defined as temperature $>38.5^{\circ} \mathrm{C}$ and positive blood culture or circulatory instability requiring intensive care monitoring), thromboembolic complications, urinary tract infection treated with antibiotics, signs of endometritis (defined as temperature of $>38,0^{\circ} \mathrm{C}$ on two occasions at least one our apart after the first $24 \mathrm{~h}$ postpartum with associated uterine tenderness), ${ }^{103}$ pneumonia, anaphylactic shock, HELLP-syndrome and death, incidence of instrumental deliveries, maternal quality of life, maternal intervention preference and costs. ${ }^{103}$

Other outcomes are direct medical and non-medical costs generated by maternal and neonatal resource utilisation during admission and post-discharge follow-up until 6 weeks after randomisation. The economic evaluation will integrate the primary clinical outcome and costs in a cost-effectiveness analysis.

\section{STATISTICAL ANALYSIS}

\section{Sample size}

This trial is designed to demonstrate a $66 \%$ reduction of neonatal sepsis from $7.5 \%$ in women in the expectant group to $2.5 \%$ in women in the induction group. This requires a total sample size of 520 patients with $80 \%$ power and a significance level of $p=0.05$.

\section{Data analysis}

Data analysis will be performed on an intention to treat basis and study baseline characteristics will be compared. The relative risks and 95\% confidence intervals will be calculated for the relevant outcome measures. The analysis will be stratified for centre and parity. Moreover, we will evaluate whether the relative benefits of induction of labour will be stronger in multiparous women and in women with a ripe cervix at baseline. In case of continuous data in the secondary outcome group, t-tests will be used whereas chi-square tests will be used if data are categorical. In case of equivalence between outcomes, the analysis will be repeated on a par protocol basis. Quality of life as well as pain scores will be analysed using repeated measures analysis of variance. ${ }^{124}$ Serious Adverse Events will be reported to an independent data safety monitoring committee. A formal interim analysis is not planned. 


\section{ETHICAL CONSIDERATIONS}

This study has been approved by the ethic committee of the University Hospital Maastricht (Ref. no. MEC 05-240). A total of about 40 academic and nonacademic hospitals have signed an intention form to participate in the trial, most of them have started the medical ethics committee approval procedure. Before a collaboration clinic may start with randomisation of patients the local ethical committee must have given their approval. The trial is registered in the controlled clinical trial register under number: ISRCTN29313500. ${ }^{125}$

\section{CONFIDENTIALLY AND DATA SECURITY}

Initials of participants as well as a local patient number are recorded in the electronic database. Linking names with patient's numbers can only be done in the local clinics. Data will be collected using Oracle Clinical Remote Data Capture (RDC), which is a new generation of application system that enables collection and cleanup of clinical trial data using the Internet. For detailed information on Oracle RDC, please visit the page of Oracle RDC products. Each participating clinic receives a login name and password to get access to the web-secured database. The access is restricted to the database of the clinic to which the password and login name belongs. Full access to the entire database is restricted to some members of the research staff.

\section{DISCUSSION}

Preterm prelabour rupture of membranes is still a clinical problem in obstetric practice. Between 34 and 37 weeks there is a lack of good clinical evidence. Induction of labour might reduce the risks of neonatal sepsis. On the other hand it might increase the incidence of instrumental deliveries and neonatal respiratory distress syndrome. This trial is designed to answer these questions in respect to neonatal and maternal outcomes and costs-effectiveness. We are aware of two other studies on the subject that are running. In Canada, a trial has been started that compares induction of labour to expectant management in women with PPROM. ${ }^{126}$ This trial aims to recruit 360 women (Safety and Efficacy Study of Intentional Delivery in Women With Preterm and Prelabour Rupture of the Membranes, Lacaze N). In St Leonards, Australia, a trial has been recently started similar to the trial that we have started (PPROMT - Preterm Prelabour Rupture Of the Membranes close to Term). ${ }^{127}$ This trial aims to recruit 1812 women and has been announced in the journal. ${ }^{128}$ The respective investigator groups, including ours, have planned an individual patient data meta-analysis after completion of the trials.

This study is funded by ZonMW (grant number 945-07-212). ${ }^{129}$ 



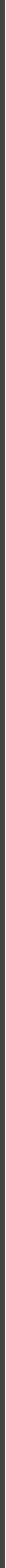




\section{CHAPTER}

\section{6 \\ INDUCTION OF LABOR VERSUS EXPECTANT MANAGEMENT IN WOMEN WITH PRETERM PRELABOR RUPTURE OF MEMBRANES BETWEEN 34 AND 37 WEEKS: \\ A RANDOMIZED CONTROLLED TRIAL}

David P van der Ham, Sylvia MC Vijgen, Jan G Nijhuis, Johannes J van Beek, Brent C Opmeer, Antonius LM Mulder, Rob Moonen, Mariët Groenewout, Mariëlle G van Pampus, Gerald D Mantel, Kitty WM Bloemenkamp, Wim J van Wijngaarden, Marko Sikkema, Monique C Haak, Paula JM Pernet, Martina Porath, Jan FM Molkenboer, Simone Kuppens, Anneke Kwee,

Michael E Kars, Mallory Woiski, Martin JN Weinans, Hajo IJ Wildschut, Bettina MC Akerboom, Ben WJ Mol, Christine Willekes, on behalf of the PPROMEXIL trial group

PLoS Med. 2012;9(4):e1001208. Epub 2012 Apr 24 


\section{ABSTRACT}

\section{BACKGROUND}

At present, there is insufficient evidence to guide appropriate management of women with preterm prelabor rupture of membranes (PPROM) near term.

\section{METHODS AND FINDINGS}

We conducted an open-label randomized controlled trial in 60 hospitals in The Netherlands, which included non-laboring women with $>24 \mathrm{~h}$ of PPROM between $34^{+0}$ and $37^{+0}$ wk of gestation. Participants were randomly allocated in a 1:1 ratio to induction of labor ( $\mathrm{OL}$ ) or expectant management (EM) using block randomization. The main outcome was neonatal sepsis. Secondary outcomes included mode of delivery, respiratory distress syndrome (RDS), and chorioamnionitis. Patients and caregivers were not blinded to randomization status. We updated a prior meta-analysis on the effect of both interventions on neonatal sepsis, RDS, and cesarean section rate. From 1 January 2007 to 9 September 2009, 776 patients in 60 hospitals were eligible for the study, of which 536 patients were randomized. Four patients were excluded after randomization. We allocated 266 women (268 neonates) to loL and 266 women ( 270 neonates) to EM. Neonatal sepsis occurred in seven (2.6\%) newborns of women in the loL group and in 11 (4.1\%) neonates in the EM group (relative risk [RR] 0.64; 95\% confidence interval $[\mathrm{Cl}] 0.25$ to 1.6). RDS was seen in 21 (7.8\%, loL) versus 17 neonates (6.3\%, EM) (RR 1.3; 95\% Cl 0.67 to 2.3 ), and a cesarean section was performed in 36 (13\%, IoL) versus 37 (14\%, EM) women (RR 0.98; 95\% Cl 0.64 to 1.50$)$. The risk for chorioamnionitis was reduced in the IoL group. No serious adverse events were reported. Updating an existing meta-analysis with our trial results (the only eligible trial for the update) indicated RRs of 1.06 (95\% $\mathrm{Cl} 0.64$ to 1.76 ) for neonatal sepsis (eight trials, 1,230 neonates) and 1.27 (95\% Cl 0.98 to 1.65) for cesarean section (eight trials, 1,222 women) for loL compared with EM.

\section{CONCLUSIONS}

In women whose pregnancy is complicated by late PPROM, neither our trial nor the updated meta-analysis indicates that IoL substantially improves pregnancy outcomes compared with EM. 


\section{INTRODUCTION}

Preterm prelabor rupture of membranes (PPROM) complicates $1 \%-5 \%$ of all pregnancies and accounts for $30 \%-40 \%$ of all preterm deliveries. ${ }^{30-32}$ It is associated with increased fetal and maternal morbidity and mortality. ${ }^{1,9,19}$

There is no consensus on the management of women with PPROM between $34^{+0}$ and $37^{+0}$ wk. The American Congress of Obstetricians and Gynecologists guidelines recommend induction of labor (IoL) if PPROM occurs at or beyond $34^{+0}$ wk of gestation. ${ }^{15}$ The Royal College of Obstetricians and Gynaecologists guidelines state that delivery should be considered at $34^{+0} \mathrm{wk}$ of gestation and recommend that women with PPROM who are managed expectantly beyond 34 wk of gestation be counseled about the increased risk of chorioamnionitis and the presumed decreased risk of neonatal respiratory problems, admission for neonatal intensive care, and cesarean section. ${ }^{16}$ The Dutch Society for Obstetrics and Gynecology guidelines advise expectant management (EM) until $35^{+0}$ wk and recommend discussing loL with the patient from $35^{+0}$ wk onwards, whereas lo $\mathrm{L}$ is advocated beyond $37^{+0}$ wk. ${ }^{5}$ Canadian and Australian surveys identify a lack of consensus on management in women with PPROM between $34^{+0}$ and $36^{+0}$ wk in those countries. ${ }^{17,18}$ A recent Cochrane review on the management of PPROM prior to $37 \mathrm{wk}$ concluded that there is insufficient evidence to guide clinical practice in the management of PPROM. ${ }^{130}$ In view of this lack of knowledge, we undertook a randomized controlled trial called PPROM Expectant Management versus Induction of Labor (PPROMEXIL). In this trial, we tested the hypothesis that IoL reduces neonatal sepsis without increasing neonatal morbidity due to prematurity and without increasing the assisted delivery rate as compared to EM in women with PPROM between $34^{+0}$ and $37^{+0}$ wk of gestation.

\section{METHODS}

We conducted a multicenter, parallel, open-label randomized controlled trial in The Netherlands, in which all eight academic and 52 non-academic hospitals participated. The study was approved by the medical ethics committee of the Maastricht University Medical Center, Maastricht, The Netherlands (Ref.no. MEC 05-240, 8 March 2006). Local approval was given by the boards of each of the participating hospitals. After the start of the trial, no changes were made to the trial protocol or to the trial outcome measures. The protocol has been published previously. ${ }^{27}$ The trial was registered in the ISRCTN register (ISRCTN29313500). This trial is reported in concordance with the CONSORT 2010 checklist. 


\section{PATIENTS}

Women with a singleton or twin pregnancy presenting with PPROM between $34^{+0}$ and $36^{+6} \mathrm{wk}$ of gestation who were not in labor within $24 \mathrm{~h}$ after rupture of membranes were eligible for this study. Women in whom PPROM was diagnosed after $26^{+0} \mathrm{wk}$, but who had not delivered by $34^{+0} \mathrm{wk}$ of gestational age were also eligible for participation. All eligible patients were counseled about participation in the study within the first $24 \mathrm{~h}$ after PPROM had occurred. Informed consent was obtained from all who participated in the study at least $24 \mathrm{~h}$ after PPROM, as soon as was possible and convenient. Breech presentation was not an exclusion criterion, as both cesarean and vaginal delivery were allowed in the protocol. Women with a monochorionic multiple pregnancy; abnormal (non-reassuring) cardiotocogram; meconium-stained amniotic fluid; signs of intrauterine infection; major fetal anomalies; hemolysis, elevated liver enzymes, and low platelets (HELLP syndrome); or severe preeclampsia were not eligible for the study. Women who declined consent for randomization but authorized use of their medical data were included in the database and were followed in the patient preference arm. Rupture of membranes was diagnosed based on history and clinical findings such as gross vaginal fluid loss, in combination with other available diagnostic test methods when necessary. The final decision on whether or not a patient had rupture of membranes was made by the attending staff. Gestational age was based either on first trimester ultrasound scan or, in women with a regular cycle, on the first day of the last menstrual period if the expected date of delivery differed less than $7 \mathrm{~d}$ from that estimated by ultrasound. In women with an unknown/uncontrolled pregnancy beyond the first trimester, gestational age was estimated by second trimester ultrasound measurements.

\section{RANDOMIZATION}

After written informed consent had been obtained, patient data were entered in a password-protected web-based database. Randomization was performed on a central password-protected web-based application developed by the clinical trial unit of the Academic Medical Center Amsterdam, Amsterdam, The Netherlands. The randomization sequence was created using a block size of four, stratified for center and parity, in a 1:1 ratio for immediate loL versus EM.

\section{Procedures}

\section{Induction of labor}

Patients allocated to loL were induced within $24 \mathrm{~h}$ after randomization. Induction was performed according to the national guidelines. ${ }^{130}$ After vaginal examination labor was induced with either prostaglandin or oxytocin. In the case of planned cesarean section, 
the cesarean section was performed as soon as feasible after randomization.

\section{Expectant management.}

Women randomized to EM were monitored according to local protocol until spontaneous delivery, which could be in an outpatient or an inpatient setting. Monitoring in both settings consisted of at least daily maternal temperature monitoring and twice weekly blood sampling for maternal leukocyte count and C-reactive protein measurement. When a patient in the EM group reached $37^{+0}$ wk of gestational age, labor was induced according to the national guidelines. ${ }^{5}$ Whenever a patient with an indication for planned cesarean section was allocated to EM, the cesarean section was performed as soon as labor commenced. Labor was induced prior to $37^{+0}$ wk of gestation if there were clinical signs of infection or when another fetal or maternal indication occurred that warranted loL.

\section{DATA COLLECTION}

At all local centers, data collection was the responsibility of the local investigators, who were supported by regional research nurses and midwives. Data were collected, coded, and processed with adequate precautions to ensure patient confidentiality. At study entry, baseline demographics and obstetric and medical history were recorded. Ethnicity and country of birth were recorded by patients themselves. Baseline blood samples were taken from all participating women at study entry. A vaginal swab was collected either at admission to the hospital or at study entry.

The national guidelines of the Dutch Society for Obstetrics and Gynecology do not indicate whether to start or not start antibiotics in women with PPROM prior to 37 wk. ${ }^{5}$ Thus, antepartum administration of antibiotics was given according to local protocol.

Postpartum neonatal and maternal outcome measures were recorded, including maternal and neonatal length of stay in hospital. The placenta was sent for histological examination to determine the presence or absence of chorioamnionitis, and funisitis in particular. Serious adverse events were reported to the Adverse Events Committee of the study. No interim analysis was planned, as both treatment options were common practice.

\section{OUTCOME MEASURES}

\section{Primary outcome}

Neonatal sepsis was defined as follows: (1) blood culture taken at birth found positive for bacteria (excluding Staphylococcus epidermidis) or (2) two or more symptoms of infection (apnea, temperature instability, lethargy, feeding intolerance, respiratory distress, hemodynamic instability) within $72 \mathrm{~h}$ after birth, plus one of the following: (a) positive blood culture (culture proven sepsis); (b) C-reactive protein $>20 \mathrm{mmol} / \mathrm{l}$ (suspicion of sep- 


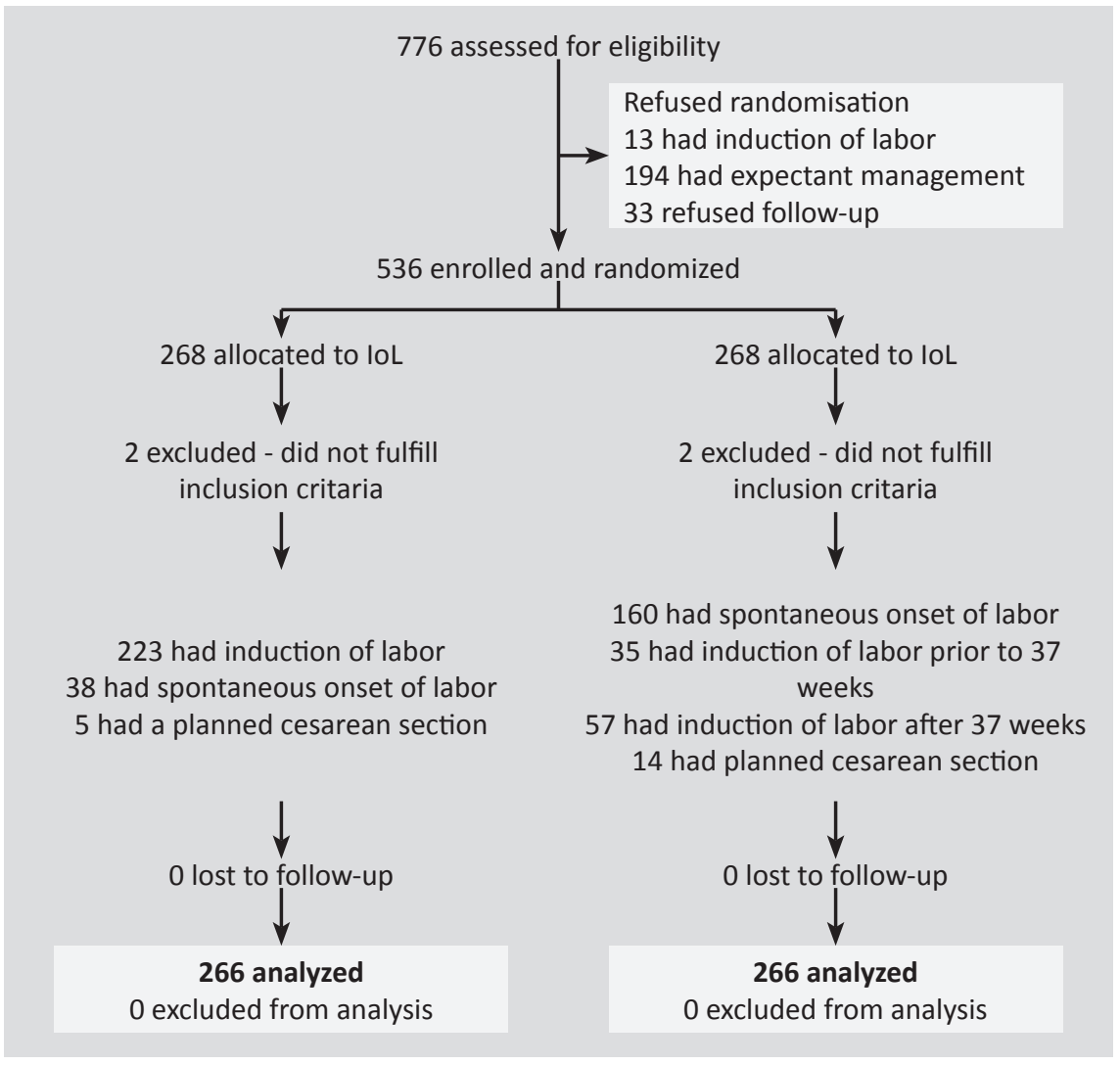

Figure 6.1 | Trial profile

sis); (c) positive surface cultures of a known virulent pathogen (suspicion of sepsis). When a local investigator classified a case as sepsis, or when criteria for sepsis were entered in the database, the case was judged by an independent panel of pediatricians (A. L. M. M. and R. M.) who, unaware of the allocation of randomization, adjudicated between neonatal sepsis (proven or suspected sepsis) or no sepsis. ${ }^{27}$

\section{Secondary outcomes}

Secondary neonatal outcome measures were respiratory distress syndrome (RDS), wet lung, meconium aspiration syndrome, pneumothorax/pneumomediastinum, asphyxia, late onset neonatal sepsis, hypoglycemia, necrotizing enterocolitis, hyperbilirubinemia, intraventricular hemorrhage, periventricular leucomalacia, convulsions, other neurological abnormalities, other complications, intrapartum death, total length of hospital stay and admission, and length of stay on neonatal intensive care unit (NICU). 
Maternal outcome measures were antepartum hemorrhage, uterine rupture, umbilical cord prolapse, signs of chorioamnionitis (defined as fever before or during labor as a temperature greater than $37.5^{\circ} \mathrm{C}$ on two occasion more than $1 \mathrm{~h}$ apart before or during labor, or a temperature greater than $38.0^{\circ} \mathrm{C}$ on one occasion with uterine tenderness, leukocytosis, maternal or fetal tachycardia, or a foul-smelling vaginal discharge in absence of any other cause of hyperpyrexia), maternal sepsis (defined as a temperature greater than $38.5^{\circ} \mathrm{C}$ and a positive blood culture or circulatory instability requiring intensive care monitoring), thromboembolic complications, urinary tract infection treated with antibiotics, endometritis (defined as a temperature greater than $38.0^{\circ} \mathrm{C}$ on two occasions at least $1 \mathrm{~h}$ apart after the first $24 \mathrm{~h}$ postpartum with associated uterine tenderness), pneumonia, anaphylactic shock, HELLP syndrome, maternal death, other complications, total length of hospital stay, and admission to the intensive care unit. Finally, we recorded mode of delivery and need for anesthesia. ${ }^{27}$

\section{STATISTICAL ANALYSIS \\ Sample size}

The proportion of neonates with sepsis was hypothesized to be $7.5 \%$ in the EM group and $2.5 \%$ in the loL group. Because it was considered clinically not plausible that loL would lead to a higher proportion of neonatal sepsis as compared to EM, we performed a power analysis based on a one-sided test, without continuity correction. This required 260 women per treatment arm to statistically demonstrate a $66 \%$ risk reduction with $80 \%$ power and a $5 \%$ type one error probability.

\section{Data analysis}

Data were analyzed on an intention to treat basis. After tabulation, study baseline characteristics were compared. Continuous data were tested with the Student's test or the non-parametric Mann-Whitney $U$ test. Relative risks (RRs), mean differences, and 95\% confidence intervals (Cls) were calculated for the relevant outcome measures. Categorical data were analyzed with $\chi^{2}$ statistics. Since the randomization was stratified for center and parity, we performed a stratified analysis using a Cochran-Mantel-Haenszel correction. The primary outcome of neonatal sepsis is presented as RR after applying the CochranMantel-Haenszel correction. Kaplan-Meier curves were constructed to analyze time from randomization to delivery in both study arms. These curves were compared using the log rank test. p-Values below 0.05 were considered to indicate statistical significance. Statistical analyses were performed using SPSS Statistics (version 17.0). 


\begin{tabular}{|c|c|c|}
\hline Characteristic $^{\mathrm{a}}$ & IoL (n=266) & $\operatorname{EM}(n=266)$ \\
\hline Maternal age (range) $[ \pm S D], y$ & $29.5(18.3-43.3)[ \pm 4.9]$ & $29.6(18.1-46.7)[ \pm 5.6]$ \\
\hline $\begin{array}{l}\text { Number nulliparous (parity range) } \\
\text { (percent nulliparous) }\end{array}$ & $147(0-5)(55 \%)$ & $152(0-5)(57 \%)$ \\
\hline Twin pregnancy & $2(0.8 \%)$ & $4(1.5 \%)$ \\
\hline \multicolumn{3}{|l|}{ Ethnic origin } \\
\hline White & 211 (79\%) & 209 (79\%) \\
\hline Other etnic origin & $35(13 \%)$ & 45 (17\%) \\
\hline Unknown & $20(7.5 \%)$ & $14(5.3 \%)$ \\
\hline \multicolumn{3}{|l|}{ Education $^{\mathrm{b}, \mathrm{c}}$} \\
\hline Primary school (4 to 12 y) & $10(6.6 \%)$ & $0(0 \%)$ \\
\hline Secondary school (12-18 y) & $11(7.2 \%)$ & $15(9.5 \%)$ \\
\hline Lower professional school & $14(9.2 \%)$ & 15 (9.5\%) \\
\hline Medium professional school & $69(46 \%)$ & $80(51 \%)$ \\
\hline Higher professional school & $35(23 \%)$ & $34(22 \%)$ \\
\hline University & $12(7.9 \%)$ & 14 (8.9\%) \\
\hline Maternal smoking & $58(23 \%)$ & $63(25 \%)$ \\
\hline $\begin{array}{l}\text { Antenatal administration of } \\
\text { corticosteroids }\end{array}$ & 37 (15\%) & $39(15 \%)$ \\
\hline \multicolumn{3}{|l|}{ Body mass index ${ }^{c}$} \\
\hline At booking (range) $[ \pm S D], \mathrm{kg} / \mathrm{m}^{2}$ & $24.8(17.0-52.2)[ \pm 5.7]$ & $24.6(16.4-45.1)[ \pm 5.1]$ \\
\hline At study entry (range) [ $\pm S D], \mathrm{kg} / \mathrm{m}^{2}$ & $29.4(16.3-52.1)[ \pm 6.3]$ & $28.7(17.9-46.3)[ \pm 5.7]$ \\
\hline \multicolumn{3}{|l|}{ Fetal position at data entry } \\
\hline Cephalic & $251(94 \%)$ & $245(92 \%)$ \\
\hline Breech & $15(5.6 \%)$ & $21(7.9 \%)$ \\
\hline
\end{tabular}

\section{Meta-AnALYSIS}

We updated a recent Cochrane review ${ }^{19}$ on sepsis, RDS, and cesarean section rate. To do so, we performed an additional search in MEDLINE and CENTRAL (from 1 October 2009 until 30 April 2011), using the same strategy as described by Buchanan et al. in order to find additional papers that were not in the systematic review. ${ }^{19}$ Two authors (D. P. v. d. H. and B. W. J. M.) identified papers for relevance and quality, and extracted the data. We calculated risk ratios, with $95 \% \mathrm{Cls}$ for all outcomes. Buchanan et al. subdivided their analysis between overall sepsis (defined or undefined by the authors) and culture-proven sepsis. ${ }^{19}$ They also made a comparison between suspicion of neonatal sepsis and management of labor. In this comparison they included one study. ${ }^{23}$ Because of the broad definition given by the authors of this study for suspicion of sepsis ("clinical findings suggestive for neonatal sepsis"), we considered our definition of suspicion of neonatal sepsis not 


\begin{tabular}{|c|c|c|}
\hline Characteristic $^{\mathrm{a}}$ (continued) & IoL (n=266) & $\operatorname{EM}(n=266)$ \\
\hline \multicolumn{3}{|l|}{ Diagnostic test for ruptur of membranes ${ }^{d}$} \\
\hline Positive history & $224(84 \%)$ & $235(88 \%)$ \\
\hline Positive ferning & $127(48 \%)$ & $133(50 \%)$ \\
\hline Positive $\mathrm{pH}$ test & $9(3.4 \%)$ & $10(3.8 \%)$ \\
\hline Positive PAMG-1 test & $17(6.4 \%)$ & $18(6.8 \%)$ \\
\hline Other positive ROM test & $18(6.8 \%)$ & $10(3.8 \%)$ \\
\hline Decrease amniotic fluid on ultrasound & $126(47 \%)$ & $133(50 \%)$ \\
\hline \multicolumn{3}{|l|}{ Gestational age at PPROM } \\
\hline$<34 w k$ & $36(14 \%)$ & $38(14 \%)$ \\
\hline $34^{+0}$ to $34^{+6} w k$ & $41(15 \%)$ & $35(13 \%)$ \\
\hline $35^{+0}$ to $35^{+6} w k$ & $79(30 \%)$ & $84(32 \%)$ \\
\hline $36^{+0}$ to $36^{+6} w k$ & $110(41 \%)$ & 109 (41\%) \\
\hline $\begin{array}{l}\text { Gestational age at randomization, } \\
\text { median [IQR], d }\end{array}$ & $251[245-255]$ & $251[245-255]$ \\
\hline $\begin{array}{l}\text { Maternal temperature at inclusion, } \\
\text { mean }[ \pm \mathrm{SD}]{ }^{\circ} \mathrm{C}\end{array}$ & $36.9[ \pm 0.48]$ & $36.9[ \pm 0.44]$ \\
\hline
\end{tabular}

Data are presented as number (percent) unless otherwise indicated.

aPercents given are related to available data per characteristic and may differ from total number of patients.

${ }^{\mathrm{b}}$ Percents given as part of known educational level.

'Outcome characteristic with more than 5\% missing data. Education: data available for 310 women (58\%); body mass index at booking: data available for 453 women (85\%); body mass index at start study available for 266 women (50\%); maternal temperature at inclusion: data available for 504 women (95\%).

${ }^{\mathrm{d}} \mathrm{S}$ um of tests exceeds $100 \%$ because more than one test could be applied on the same patient. EM, expectant management; IoL, induction of labor; IQR, interquartile range; PAMG-1, placental alpha microglobulin-1; ROM, rupture of membranes; SD, standard deviation.

Table 6.1 | Baseline characteristics

comparable. But we considered our overall sepsis rate as comparable with the overall sepsis rate, and added the culture-proven sepsis cases in our study to the cultureproven sepsis comparison in the meta-analysis. Statistical analyses were carried out using RevMan, version 5.1.131

\section{RESULTS}

From 1 January 2007 until 9 September 2009 a total of 776 women were asked to participate in the trial, of which 536 women (69\%) gave informed consent. A total of 268 women were randomized to IOL (IoL group) and 268 to EM (EM group). Figure 6.1 outlines the study profile. In both arms two patients were excluded because after 


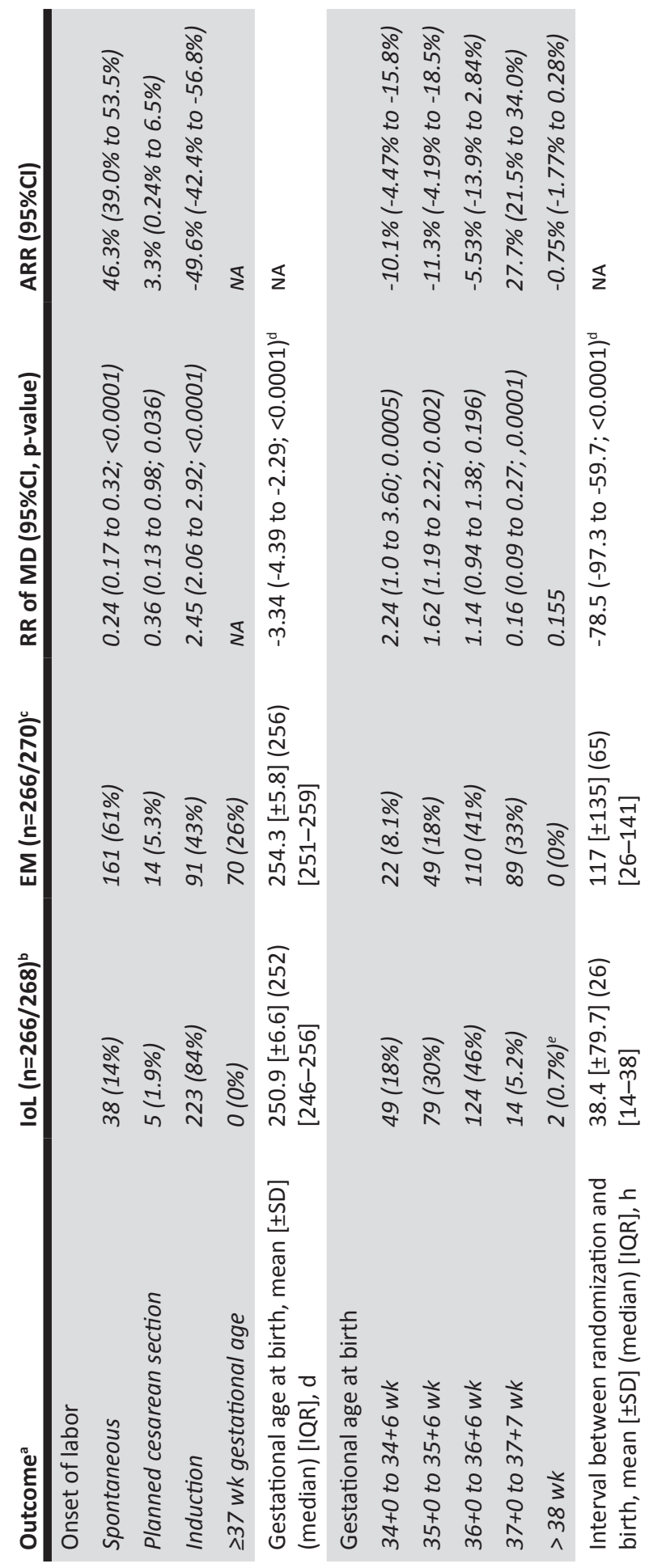




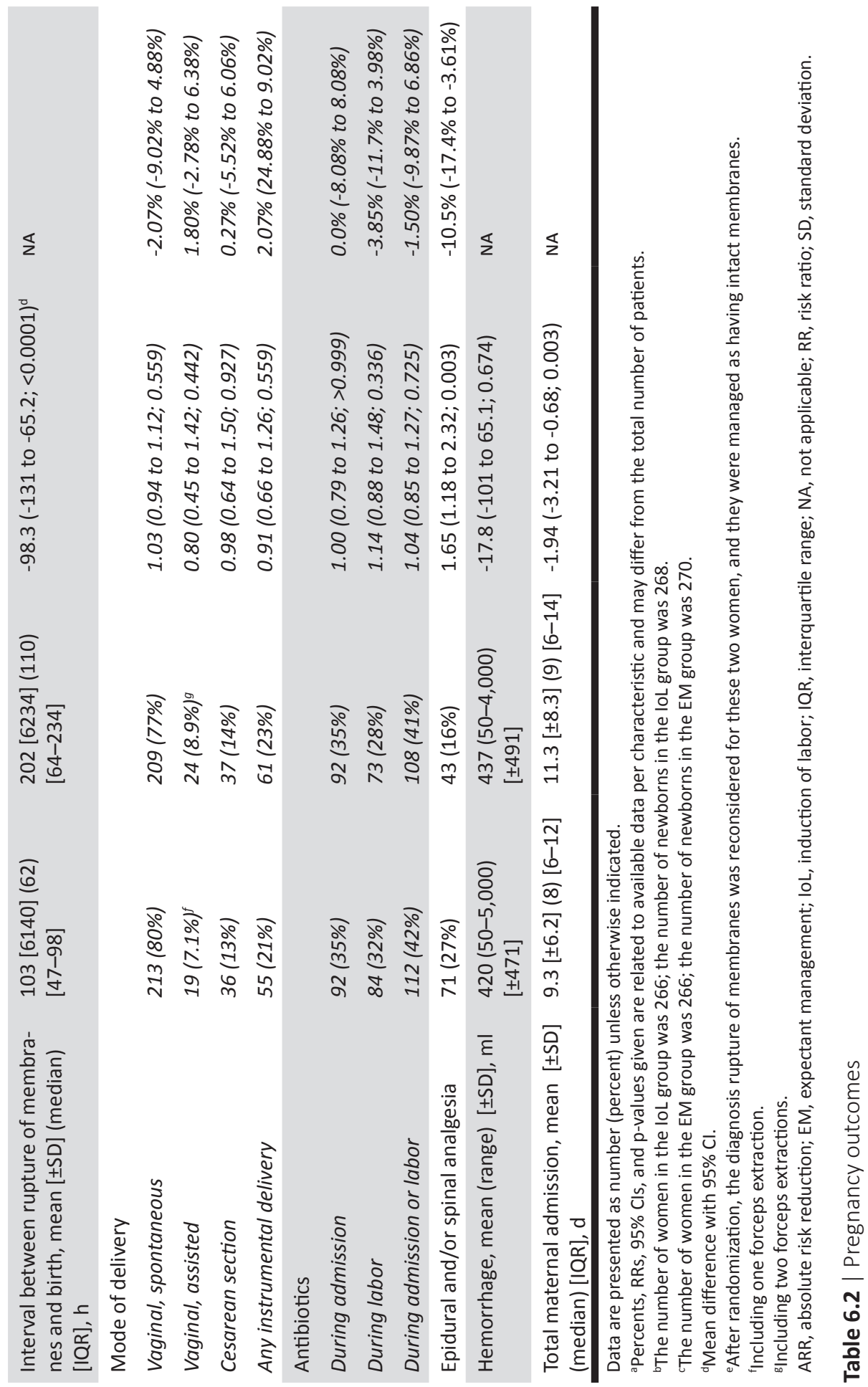




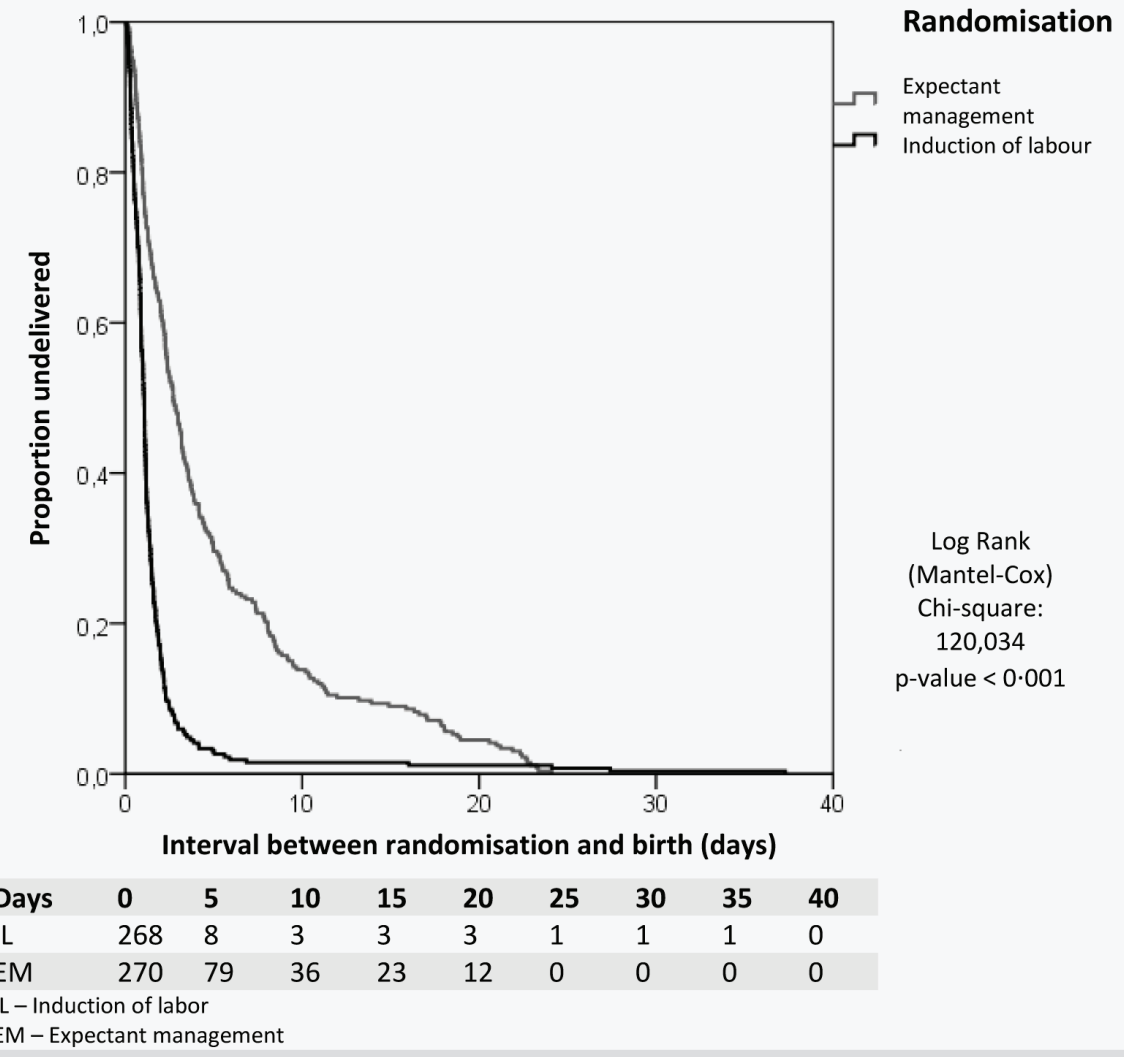

Figure 6.2 | Kaplan-Meier curve for the interval between randomization and birth

completion of the trial it became clear that their gestational age was over $36^{+6} \mathrm{wk}$ at the time of inclusion. Baseline characteristics were comparable between the two groups (Table 6.1). Median gestational age at randomization was $251 \mathrm{~d}\left(35^{+6} \mathrm{wk}\right)$. The percentage of women that had PPROM before 34 wk of gestation was $14 \%$ in both groups. Table 6.2 shows the data on pregnancy outcome and mode of delivery. In the EM group, labor was induced in 94 women (34\%), in 70 (77\%) of these cases because the gestational age of 37 wk was reached. In 24 women induction was prior to $37 \mathrm{wk}$, in four cases (4.4\%), this was for fetal distress, in four (4.4\%) for meconium-stained amniotic fluid, in six (6.6\%) for signs of infections, in three (3.3\%) for maternal hypertensive disorders, and in two $(2.2 \%)$ for other maternal complications. In the remaining two patients (2.2\%), no reason for induction was recorded. Figure 6.2 shows the Kaplan-Meier curve for the interval between randomization and delivery in both groups.

Of the 266 women allocated to the loL group, 38 (14\%) went into labor spontaneously (i.e., after randomization but before it was possible to start induction), five (2\%) had a 
planned cesarean section, and in the remaining 223 (84\%) women, labor was induced. Women allocated to loL obtained epidural or spinal analgesia more often than those in the EM group, 71 (27\%) versus 43 (16\%), respectively (RR 1.7; 95\% Cl 1.2 to 2.3). There was no difference in antibiotic administration during admission or labor between the two groups. The cesarean section rate was comparable in both groups (36 [13\%] cesarean sections in the loL group versus 37 [14\%] in the EM group; RR 0.98; 95\% Cl 0.64 to 1.5). No serious adverse events were reported during the study period.

\section{NEONATAL SEPSIS}

Neonatal sepsis was seen in seven newborns (2.6\%) in the loL group versus $11(4.1 \%)$ in the EM group (RR 0.64; 95\% Cl 0.25 to 1.6) (see Table 6.3). The RR for neonatal sepsis was similar when stratified for center and parity (RR $0.65 ; 95 \% \mathrm{Cl} 0.25$ to 1.7 ).

\section{OTHER NEONATAL OUTCOMES}

Table 6.3 shows all neonatal outcomes. Neonates born in the loL group stayed $1.4 \mathrm{~d}$ longer in hospital than neonates born after EM and were admitted more often to the NICU. Newborns admitted to the NICU in the loL group stayed shorter than those in the EM group. RDS was seen in 21 (7.8\%) newborns in the loL group versus 17 (6.3\%) in the EM group. Hypoglycemia (RR 2.2) and hyperbilirubinemia (RR 1.5) were seen significantly more often in the loL group. For other neonatal outcome measures, there were no significant differences between the two groups. Seventy-six newborns (28\%) in the loL group were treated with antibiotics, for an average of $5.0 \mathrm{~d}$, versus $78(27 \%)$ in the EM group, for an average of $5.0 \mathrm{~d}$ (Table 6.4).

\section{MATERNAL OUTCOMES}

Table 6.5 shows the maternal outcomes. Clinical chorioamnionitis was seen in six women (2.3\%) in the loL group versus 15 (5.6\%) women in the EM group (RR 0.40; 95\% Cl 0.16 to 1.02). The incidence of histological chorioamnionitis was 43 (22\%) versus 62 (32\%), respectively (RR 0.69; $95 \% \mathrm{Cl} 0.49$ to 0.96 ).

\section{NON-RANDOMIZED WOMEN}

Table 6.6 shows the baseline characteristics of the 207 nonrandomized women. The nonrandomized women differed to those randomized in level of education (more educated), smoking (fewer smoked), maternal age (older), and management (preferred EM). Furthermore, they differed in gestational age at PPROM (earlier PPROM). Neonatal sepsis was seen in one of 13 (7.7\%) neonates born to mothers who preferred loL, and in seven of the 198 (3.5\%) neonates born to mothers who chose EM. 


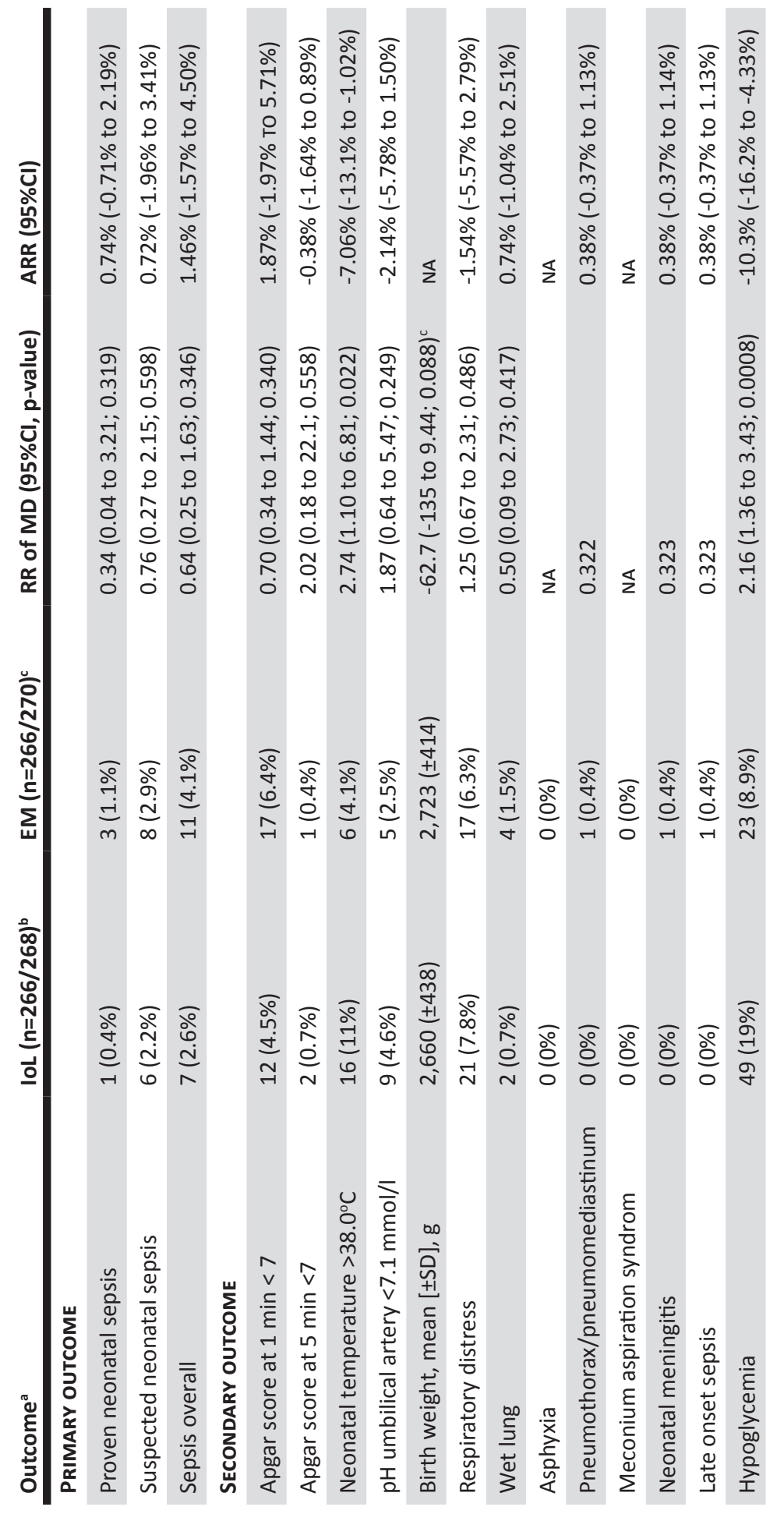



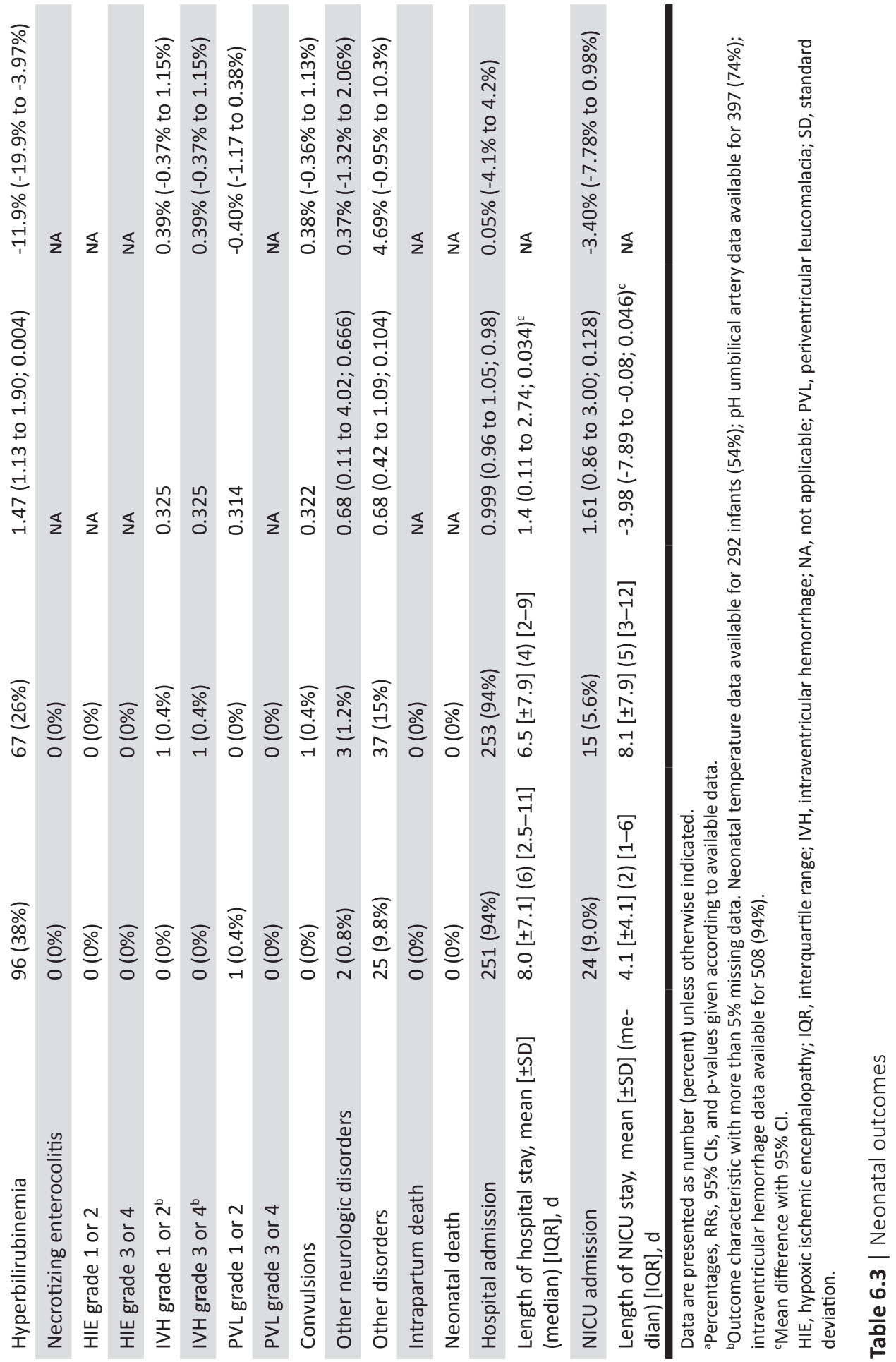


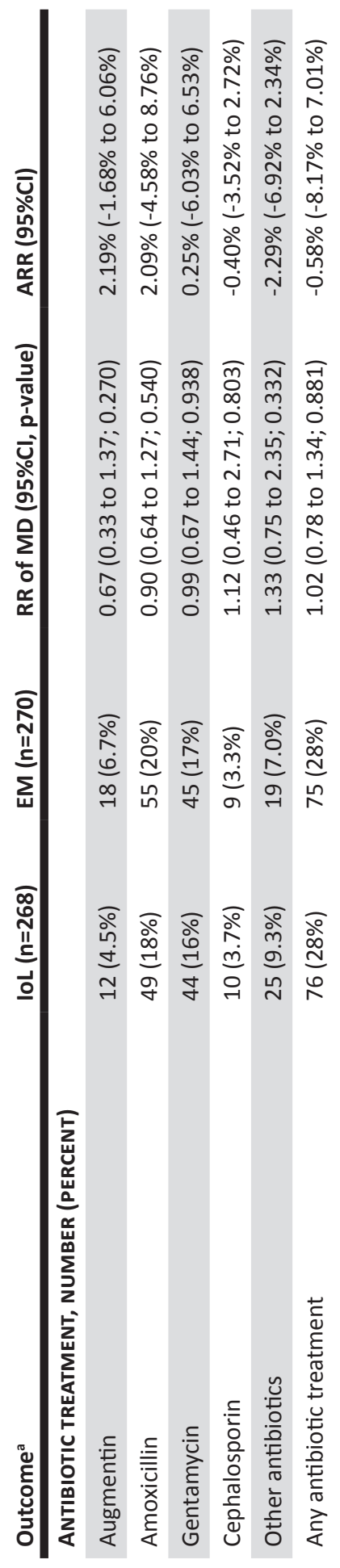




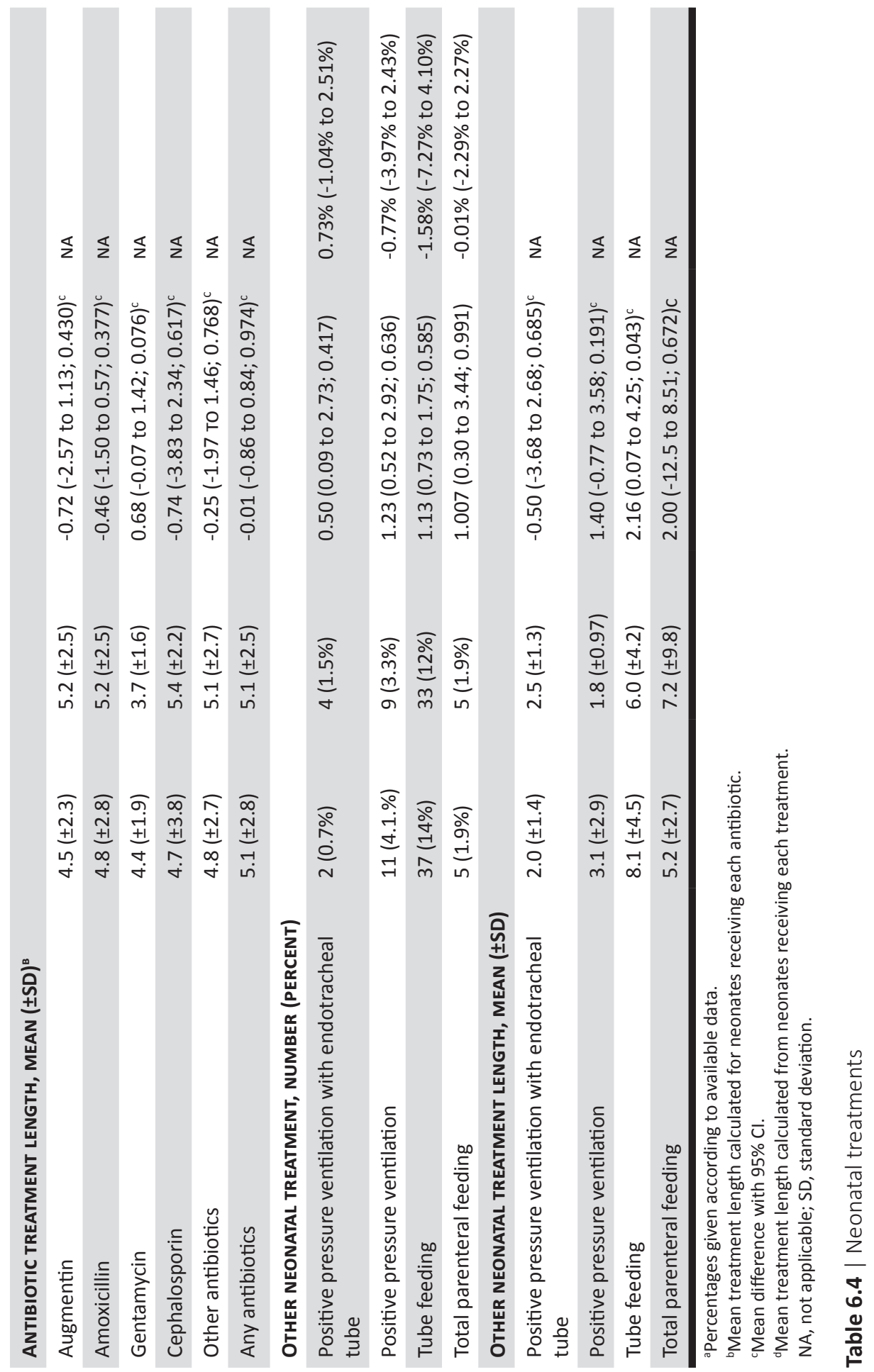




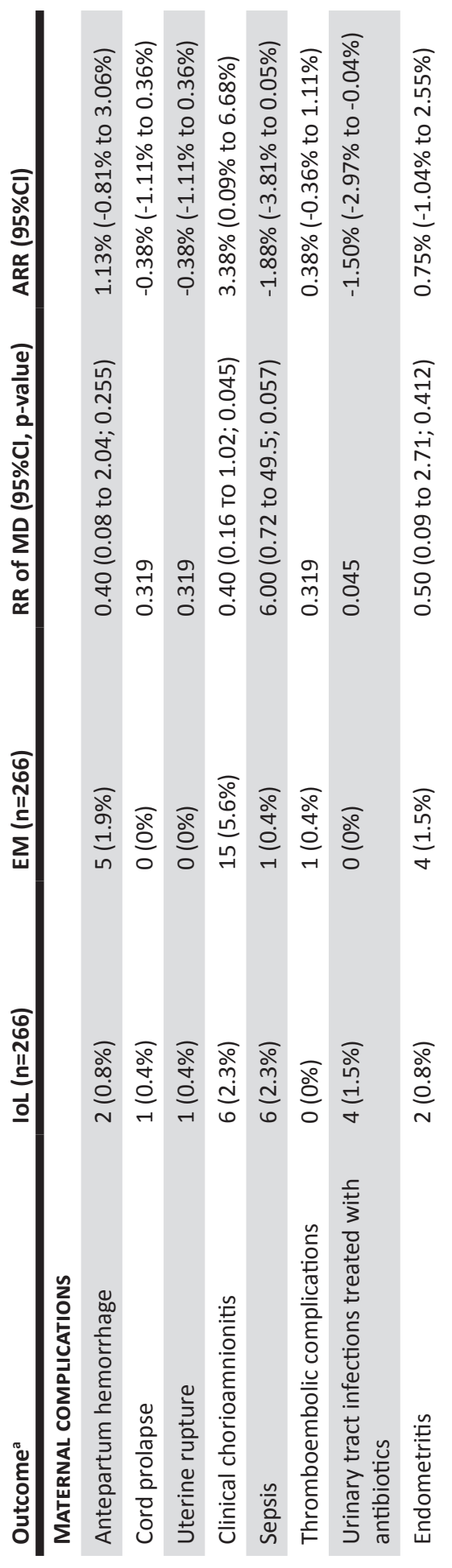



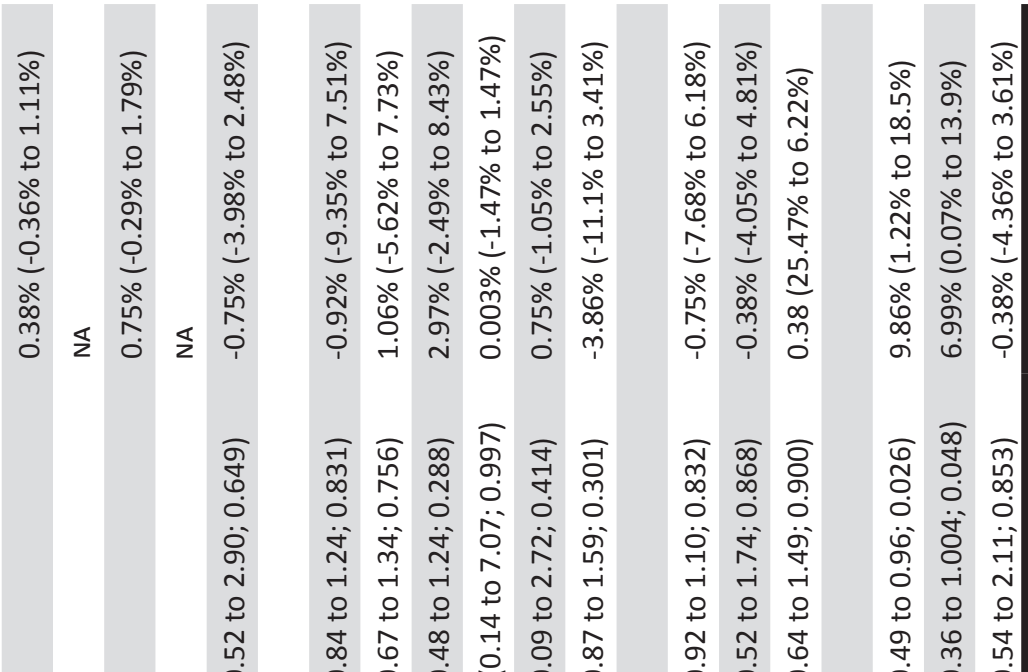

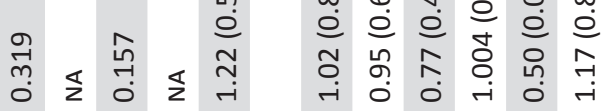

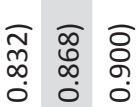

ஸ்

तi

웅유

ศิ กิ ซี

ㅇํ

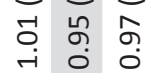

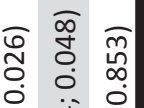

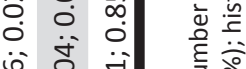

ศุ ठ

○

ค

仓

Øิ

ह.일

㐫这

㠃 $\frac{0}{\frac{0}{\pi}}$

कृ

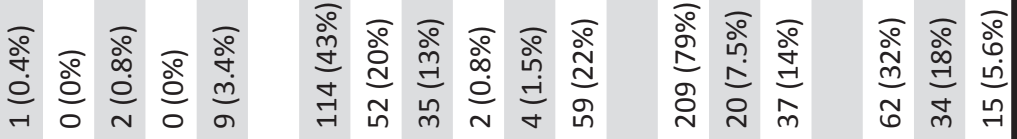

:

造

है

$\frac{5}{\frac{5}{5}}$

응

产 


\begin{tabular}{|c|c|c|c|}
\hline Characteristic $^{a}$ & $\begin{array}{l}\text { Randomized } \\
(n=532)\end{array}$ & $\begin{array}{l}\text { Non-Randomized } \\
(\mathrm{n}=207)\end{array}$ & p-value \\
\hline Maternal age (range) [ \pm SD], y & $\begin{array}{l}29.6(18.0-46.7] \\
( \pm 5.3]\end{array}$ & $\begin{array}{l}32.3(19.5-45.5) \\
{[ \pm 5.0]}\end{array}$ & $<0.0001$ \\
\hline $\begin{array}{l}\text { Number nulliparous (parity range) } \\
\text { (percent nulliparous) }\end{array}$ & $299(0-5)(56 \%)$ & $121(0-3)(58 \%)$ & 0.580 \\
\hline Twin pregnancy & $6(1.1 \%)$ & 4 (1.9\%) & 0.395 \\
\hline \multicolumn{4}{|l|}{ Ethnic origin } \\
\hline White & 420 (79\%) & $149(72 \%)$ & \multirow{3}{*}{0.188} \\
\hline Other etnic origin & $78(15 \%)$ & $37(18 \%)$ & \\
\hline Unknown & $34(6.4 \%)$ & 21 (10\%) & \\
\hline \multicolumn{4}{|l|}{ Education ${ }^{\mathrm{b}, \mathrm{c}}$} \\
\hline Primary school (4 to 12 y) & $10(3.2 \%)$ & $0(0 \%)$ & \multirow{6}{*}{0.002} \\
\hline Secondary school (12-18 y) & $26(8.4 \%)$ & $10(8.7 \%)$ & \\
\hline Lower professional school & $29(9.4 \%)$ & $5(4.4 \%)$ & \\
\hline Medium professional school & 149 (48\%) & $42(37 \%)$ & \\
\hline Higher professional school & 70 (23\%) & $37(32 \%)$ & \\
\hline University & $26(8.4 \%)$ & $21(18 \%)$ & \\
\hline Maternal smoking & $121(24 \%)$ & $21(11 \%)$ & 0.001 \\
\hline \multicolumn{4}{|l|}{ Body mass index ${ }^{c}$} \\
\hline At booking (range) $[ \pm S D], \mathrm{kg} / \mathrm{m}^{2}$ & $\begin{array}{l}24.7(16.4-52.2) \\
{[ \pm 5.4]}\end{array}$ & $\begin{array}{l}24.4(17.3-41.9) \\
{[ \pm 4.5]}\end{array}$ & 0.605 \\
\hline
\end{tabular}

\section{Meta-AnAlysis}

The electronic search yielded ten new results relevant for meta-analysis. After reviewing these papers, none fulfilled the inclusion criteria. Figure 6.3 presents the results of the meta-analysis including our own data. In total, 1,230 neonates could be analyzed from eight studies for neonatal sepsis, 892 neonates (five studies) for culture-proven sepsis, 1,230 neonates (eight studies) for RDS, and 1,222 women (eight studies) for cesarean section rate. None of the risk ratios for these outcomes were statistically different (1.06 [95\% $\mathrm{Cl} 0.64-1.76$ ], $p=0.81 ; 0.94$ [95\% Cl 0.43-2.05], $p=0.87 ; 1.03$ [95\% Cl 0.80-1.33], $p=0.83$; 1.27 [95\% Cl 0.98 to 1.65$], p=0.07$, respectively).

\section{DISCUSSION}

We conducted a large randomized study (the PPROMEXIL trial) to compare IoL and EM in women with PPROM between 34 and 37 wk of gestational age. Because of the conservative treatment policy and conservative preferences amongst patients in The Netherlands, 


\begin{tabular}{|c|c|c|c|}
\hline $\begin{array}{l}\text { Characteristic }^{a} \\
\text { (continued) }\end{array}$ & $\begin{array}{l}\text { Randomized } \\
(n=532)\end{array}$ & $\begin{array}{l}\text { Non-Randomized } \\
(n=207)\end{array}$ & $p$-value \\
\hline At study entry (range) [ $\pm S D], \mathrm{kg} / \mathrm{m}^{2}$ & $\begin{array}{l}29.1(16.3-52.1) \\
{[ \pm 6.0]}\end{array}$ & $\begin{array}{l}28.0(17.3-43.8) \\
{[ \pm 4.7]}\end{array}$ & 0.139 \\
\hline \multicolumn{4}{|l|}{ Gestational age at PPROM } \\
\hline$<34 w k$ & 74 (14\%) & 60 (29\%) & \multirow{4}{*}{$<0.0001$} \\
\hline $34^{+0}$ to $34^{+6} w k$ & $76(14 \%)$ & $38(18 \%)$ & \\
\hline $35^{+0}$ to $35^{+6} w k$ & 163 (31\%) & 64 (31\%) & \\
\hline $36^{+0}$ to $36^{+6} w k$ & $219(41 \%)$ & $44(21 \%)$ & \\
\hline $\begin{array}{l}\text { Gestational age at PPROM, median } \\
{[I Q R], d}\end{array}$ & 249 [243-253] & 244 [234-250] & $<0.0001$ \\
\hline $\begin{array}{l}\text { Maternal temperature at inclusion, } \\
{[ \pm \mathrm{SD}] .{ }^{\circ} \mathrm{C}^{\mathrm{c}}}\end{array}$ & $36.9[ \pm 0.46]$ & $36.8[ \pm 0.46]$ & 0.214 \\
\hline \multicolumn{4}{|l|}{ Treatment } \\
\hline IoL & 266 (50\%) & $13(6.3 \%)$ & \multirow{2}{*}{$<0.0001$} \\
\hline$E M$ & 266 (50\%) & 194 (94\%) & \\
\hline \multicolumn{4}{|c|}{$\begin{array}{l}\text { Data are presented as number (percent) unless otherwise indicated. } \\
\text { apercents given are related to available data per characteristic and may differ from the total number of } \\
\text { patients. } \\
\text { bPercents given as part of known educational level. } \\
\text { 'Outcome characteristic with more than } 5 \% \text { missing data. Education: data available for } 425 \text { women } \\
\text { ( } 58 \%) \text {; body mass index at booking (BMI): data available for } 616 \text { women ( } 83 \%) \text {; BMl at start study avai- } \\
\text { lable for } 346 \text { women ( } 47 \%) \text {; maternal temperature at inclusion: data available for } 689 \text { women ( } 93 \%) \text {. }\end{array}$} \\
\hline
\end{tabular}

Table 6.6 | Baseline characteristics for randomized versus non-randomized participants

we had an ideal population in which to perform this trial, with extensive data on all eligible patients including nonparticipants (31\%), the vast majority of whom declined participation because they preferred EM. We found that in pregnancies complicated by PPROM between 34 and 37 wk of gestation, IoL does not substantially reduce the incidence of neonatal sepsis compared to EM. The number of neonates with respiratory distress was comparable in both arms, and IoL did not increase the risk of a cesarean section, findings that were confirmed in meta-analysis. However, in our study loL increased the risk of hypoglycemia and hyperbilirubinemia, as well as the use of epidural or spinal analgesia during labor. Our findings are in line with the results of the TERMPROM trial, which compared IOL with EM in 5,041 women with prelabor rupture of membranes at term. ${ }^{3}$ The TERMPROM trial showed that loL did not reduce the risk of neonatal sepsis as compared to $\operatorname{EM}(2.5 \%$ versus $2.8 \%){ }^{3}$

In contrast to earlier studies, 3,23,24,118 our pragmatic protocol did not include routine cultures from all neonates to diagnose sepsis. Because of the lack of consensus amongst 


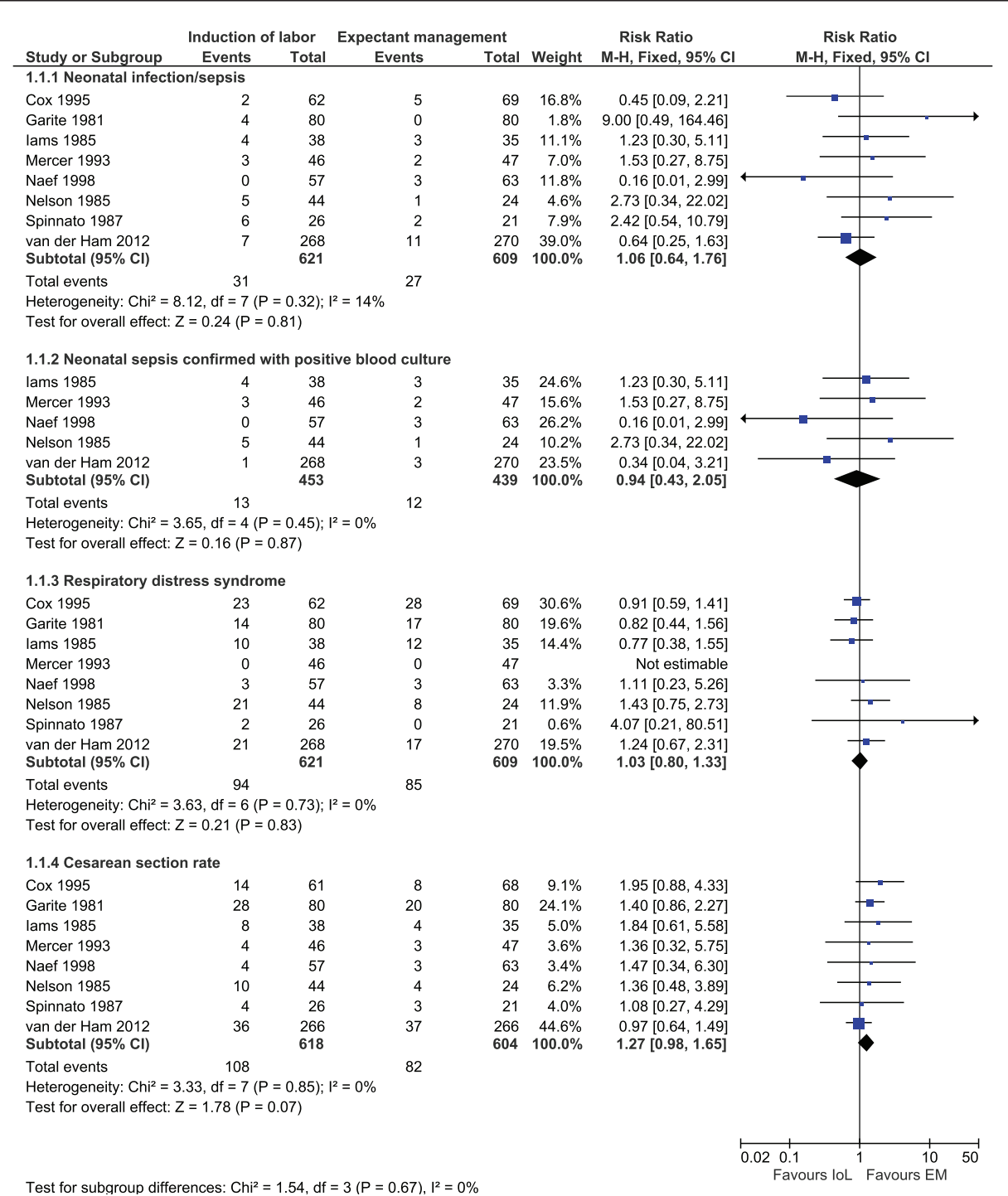

Figure 6.3 | Neonatal outcomes

Risk ratio according to Mantel-Haenszel (M-H) with fixed effects and 95\% Cls for neonatal sepsis, culture-proven neonatal sepsis, RDS, and cesarean section rates.

Dutch neonatologists on whether to take blood samples routinely after prolonged premature rupture of membranes, neonatal blood samples and liquor cultures were taken only on clinical indication. All cases with any possible sign of neonatal sepsis were adjudicated by a panel of neonatologists. In consensus they decided whether or not a newborn had suffered neonatal sepsis (suspected or proven). Despite of the lack of blood culture from 
all neonates in the trial, we believe that no cases of neonatal sepsis were missed and that the incidence of neonatal sepsis was not overestimated.

IoL reduced the risk of chorioamnionitis. Several studies have demonstrated a relationship between chorioamnionitis and adverse neonatal outcome. ${ }^{132-135}$ In a large study of very premature neonates ( $<28$ wk; the ELGAN study), ${ }^{134}$ a relationship between cerebral palsy and/or white matter damage and positive bacteriological cultures from the placenta was demonstrated. Other studies have also described a relationship between chorioamnionitis and increased risk for sepsis, respiratory distress, pneumonia, and even neonatal death. ${ }^{132,133}$ We doubt, however, that these findings can be extrapolated to our population. The incidence of cerebral palsy is significantly lower in the near-term-birth population, and reported incidences of adverse neonatal outcome in a near-term and term newborns are low (maximum reported incidences of 1.9\% in the chorioamnionitis group). ${ }^{132}$ In line with the TERMPROM trial, the number of cesarean sections in our study was comparable in the IoL and EM groups. ${ }^{3}$ We could not confirm the trend for increased risk on cesarean section in the EM group that was reported in the previous systematic review. ${ }^{19}$ The risks of hypoglycemia and hyperbilirubinemia were decreased in children of women treated expectantly. These findings have, to our knowledge, not previously been reported for a prospective study. In a recent retrospective study in three tertiary hospitals in France, a similar incidence of hyperbilirubinemia (37\% for loL versus 33\% for EM) and a slightly lower incidence of hypoglycemia (12\% for loL versus $6 \%$ for EM) was found, but due to a lack of power, differences were not statistically significant in the French study. ${ }^{136}$ We doubt whether prolongation of pregnancy, which was on average $3.3 \mathrm{~d}$ longer in the EM group in our trial, will have solely contributed to the decreased risk of hypoglycemia and hyperbilirubinemia. Maybe spontaneous onset of labor enhances the speed of physiological maturing by means of a still unknown adaptational process, as is known to happen in the lungs, reducing the incidence of RDS in spontaneous delivery compared to elective cesarean section. ${ }^{137}$

The clinical importance of these findings for later (cognitive and motor) development in children is not clear at present for our study group, although it is known that following symptomatic neonatal hypoglycemia, more than $50 \%$ of infants demonstrate cognitive and motor impairments at the age of 18 mo. ${ }^{138}$ In lowbirth-weight infants, even an asymptomatic moderate hypoglycemia may lead to cognitive and motor impairments at the age of 18 mo. ${ }^{139}$ Hyperbilirubinemia is potentially neurotoxic, especially in infants born preterm. ${ }^{140}$ When treated appropriately, however, bilirubin levels under $30 \mathrm{mg} / \mathrm{dl}$ are not associated with adverse neurodevelopmental outcome. ${ }^{141}$

Mackay et al. ${ }^{142}$ reported on the increased need for special education in preterm-delivered infants. In a retrospective cohort study of 407,503 schoolchildren, they showed 
that gestational age at delivery had a strong, dose-dependent relationship with special educational need. Until further evidence becomes available, the decreased risk of special educational need with advancing gestational age should be taken into account when considering how best to manage PPROM.

Within the Dutch Consortium for Women's Health and Reproductivity Studies (http:// www.studies-obsgyn.nI), the PPROMEXIL trial is the largest trial so far with regard to the number of participating hospitals (60 out of 98 eligible hospitals, 61\%). The 207 nonrandomized women in our study who allowed data collection differed from the randomized women. Similar to two other Dutch consortium trials (HYPITAT and DIGITAT), ${ }^{143,144}$ the women who agreed to be randomized differed in level of education, smoking habits, maternal age, and preferred management from those who did not agree to be randomized. The non-randomized subgroup of women who preferred loL was too small to draw any conclusions from. In the EM subgroup, no differences were seen in the primary outcome or the secondary neonatal and maternal outcomes. Even though some women eligible to participate in the trial did not, we believe that we did not miss a significant group at a higher (or lower) risk for neonatal sepsis who were treated expectantly. Despite some differences in baseline characteristics, we assume that the results of our study can be generalized to at least the Dutch/Western European population. Because of wide differences in general health care and availability of antibiotics, it is likely that these results cannot be generalized to low-income countries.

The main limitation of our study is that it proved to be underpowered. We hypothesized a decrease in neonatal sepsis rate of $7.5 \%$ to $2.5 \%$, but found a difference of only $1.5 \%$ (2.6\% in the loL group versus $4.1 \%$ in the EM group). The liberal use of antibiotic therapy before or during labor ( $41 \%$ of all participating women received antibiotics) might have contributed to a lower incidence compared to the other trials in which antibiotics were not administered prophylactically. ${ }^{20-26}$ These previous studies showed high rates of neonatal sepsis with EM. Similarly, improved health care may have contributed to a reduction of the incidence of neonatal sepsis in women with PPROM over the last decades.

In our study the observed difference in sepsis rates between the EM and IoL groups did not reach statistical significance. Although this study is one of the largest published so far, our sample size was insufficient to demonstrate small differences. In retrospect, anticipating a risk reduction of 66\% (a difference in neonatal sepsis rate of $7.5 \%$ versus $2.5 \%$ ) might have been too optimistic. However, several previous studies $20,22,24$ showed neonatal sepsis incidences up to $9.5 \%$ with EM, and we did observe an incidence near $2.5 \%$ in the loL group. Although optimistic, we feel that our hypothesized risk reduction was not unrealistic. Furthermore, we used a one-sided test for the power calculation. We considered it not plausible that IoL in women with PPROM near term would increase the proportion 
of cases of neonatal sepsis. In retrospect, considering the results of the meta-analysis, one might question this choice of a one-sided test, as several studies in the meta-analysis show an increased risk for sepsis in the loL group. However, the analysis was executed exactly as planned in advance. Two-sided testing would have required a sample size that would not have been feasible in our setting, in view of the limitations set by our funding body.

A second potential limitation is that EM prolonged gestation by just $4 \mathrm{~d}$. This rather small difference might be partly due to the fact that the median gestational age at rupture of membranes was $35^{+4} \mathrm{wk}$ and median gestational age at randomization was $35^{+6} \mathrm{wk}$. The overrepresentation of women beyond the 35th completed week of gestation was caused by the fact that women in their 35th week of gestation more often refused to participate (mean gestational age at PPROM in the non-randomized group was $34^{+6} \mathrm{wk}$ ), and before our study an expectant policy was the standard. Furthermore, hesitation of clinicians to induce labor before 35 completed weeks of gestation, which prior to the start of the PPROMEXIL trial was not recommended in the Dutch guidelines, might also have influenced this outcome.

A third limitation of this study is that we reported many secondary, mostly neonatal, outcomes. Although this is not uncommon in studies in maternal-fetal medicine, it is possible that a significant difference can be found by chance. If one applies a Bonferroni correction to the $p$-value, the adjusted threshold is $p<0.001$. By applying this threshold, the incidence of hypoglycemia $(p=0.0008)$ remains the only statistically significant difference between the groups.

We are aware of the ongoing multicenter PPROMT trial (ISRCTN44485060), which has a design similar to that of our study. That trial may possibly answer the question whether IoL in women with near-term PPROM reduces the risk of neonatal sepsis. ${ }^{128}$ However, the updated meta-analysis clearly demonstrates that the incidence of neonatal sepsis is comparable in both treatment strategies. ${ }^{128}$ We therefore plan to perform an individual patient data meta-analysis on the management of PPROM. Combining large trials in an individual patient data meta-analysis would, in our opinion, produce the best currently available evidence for the management of PPROM. We have already planned such an analysis with the PPROMT study group, and will contact researchers from other published trials to collaborate in an individual patient data meta-analysis.

We conclude that in pregnancies complicated by PPROM between 34 and 37 wk of gestation the incidence of neonatal sepsis is low. Neither our trial nor the updated metaanalysis shows that IoL substantially improves pregnancy outcomes compared with EM. 


\section{EDITORS' SUMMARY}

\section{BACKGROUND}

Most pregnancies last around 40 weeks, but in industrialized countries, 5\%-10\% of babies are born before 37 weeks of gestation (gestation is the period during which a baby develops in its mother's womb). Premature birth is a major cause of infant death in many developed countries, and preterm babies can also have short- and/or long-term health problems such as breathing problems, increased susceptibility to life-threatening infections, and learning and developmental disabilities. There are many reasons why some babies are born prematurely, but preterm prelabor rupture of the membranes (PPROM) accounts for $30 \%-40 \%$ of preterm deliveries. Inside the womb, the baby is held in a fluid-filled bag called the amniotic sac. The amniotic fluid cushions the baby, helps some of its organs develop, and protects both mother and baby from infection. The membranes that form the sac usually break at the start of labor ("water breaking"), but in PPROM, the membranes break before the baby is fully grown. PPROM increases the mother's risk of a womb infection called chorioamnionitis and the baby's risk of neonatal sepsis (blood infection), and can trigger early labor.

\section{WHY WAS THIS STUDY DONE?}

There is currently no consensus on how to manage women whose membranes rupture between 34 and 37 weeks' gestation. Some guidelines recommend immediate induction of labor if PPROM occurs at or beyond 34 weeks' gestation. Others recommend that labor not be induced unless the mother develops signs of infection such as a high temperature or has not delivered her baby spontaneously by 37 weeks' gestation (expectant management). Before 34 weeks' gestation, expectant management is generally recommended. In this randomized controlled trial, the researchers compare the effects of induction of labor and of expectant management on the rate of neonatal sepsis (the proportion of babies that develop neonatal sepsis; the trial's primary outcome) and on secondary outcomes such as the rates of neonatal respiratory distress syndrome (RDS), cesarean section (surgical delivery), and chorioamnionitis in women with PPROM between 34 and 37 weeks' gestation. The researchers also undertake a metaanalysis of published trials on the effect of both interventions on pregnancy outcomes. A randomized controlled trial compares the effects of different interven- 
tions in groups of individuals chosen through the play of chance; meta-analysis is a statistical approach that combines the results of several trials.

\section{WHAT DID THE RESEARCHERS DO AND FIND?}

In the PPROM Expectant Management versus Induction of Labor (PRROMEXIL) trial, 532 non-laboring women with PPROM between 34 and 37 weeks' gestation were randomly assigned to either immediate induction of labor or expectant management. Neonatal sepsis occurred in seven babies born to women in the induction of labor group and in 11 babies born to women in the expectant management group. This difference was not statistically significant. That is, it could have happened by chance. Similarly, although more babies born to women in the induction of labor group than in the expectant management group developed RDS ( 21 and 17 babies, respectively), this difference was not significant. Cesarean section rates were similar in both intervention groups, but the risk of chorioamnionitis was slightly reduced in the induction of labor group compared to the expectant management group. Finally, the researchers' meta-analysis (which included these new results) found no significant differences in the risk of neonatal sepsis, RDS, or cesarean section associated with the two interventions.

\section{WHAT DO THESE FINDINGS MEAN?}

These findings show that, compared to expectant management, induction of labor did not reduce the incidence of neonatal sepsis in pregnancies complicated by PPROM between 34 and 37 weeks' gestation. However, because fewer babies than expected born to the women in the expectant management group developed neonatal sepsis, this trial was underpowered. That is, too few women were enrolled in the trial to enable the detection of a small difference between the interventions in the neonatal sepsis rate. These findings also show that induction of labor did not substantially affect most of the secondary outcomes measured by the researchers. Given these results and those of their meta-analysis, the researchers conclude that, in women whose pregnancy is complicated by PPROM late in pregnancy, induction of labor does not substantially improve the outcome for either the woman or her baby compared to expectant management. 


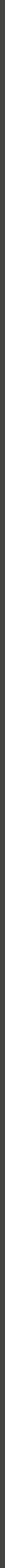




\section{CHAPTER}

\section{7 \\ MANAGEMENT OF LATE PRETERM PREMATURE RUPTURE OF MEMBRANES: THE PPROMEXIL-2 TRIAL}

David P van der Ham; Jantien L van der Heyden; Brent C Opmeer; Antonius LM Mulder; Rob MJ Moonen; J (Hans) J van Beek;

Maureen TM Franssen; Kitty WM Bloemenkamp; J (Marko) M Sikkema; Christianne JM de Groot; Martina Porath; Anneke Kwee; Mallory D Woiski; J (Hans) J Duvekot; Bettina MC Akerboom; Aren J van Loon; Jan W de Leeuw; Christine Willekes; Ben WJ Mol; Jan G Nijhuis

\section{- EDITORS' CHOICE -}

Am J Obstet Gynecol. 2012 Oct;207(4):276.e1-276.e10. doi: 10.1016/j. ajog.2012.07.024. Epub 2012 Jul 20. 


\section{ABSTRACT}

\section{Objective}

The evidence for the management of near term prelabor rupture of membranes is poor. From January 2007 until September 2009, we performed the PPROM Expectant Management versus Induction of Labor (PPROMEXIL) trial. In this trial, we showed that in women with preterm prelabor rupture of membranes (PPROM), the incidence of neonatal sepsis was low, and the induction of labor (loL) did not reduce this risk. Because the PPROMEXIL trial was underpowered and because of a lower-than-expected incidence of neonatal sepsis, we performed a second trial (PPROMEXIL-2), aiming to randomize 200 patients to improve the evidence in near-term PPROM.

\section{STUDY DESIGN}

In a nationwide multicenter study, nonlaboring women with PPROM between 34 and 37 weeks' gestational age were eligible for inclusion. Patients were randomized to loL or expectant management (EM). The primary outcome measure was neonatal sepsis.

\section{RESULTS}

From December 2009 until January 2011, we randomized 100 women to loL and 95 to EM. Neonatal sepsis was seen in 3 neonates (3.0\%) in the loL-group versus 4 neonates (4.1\%) in the EM group (relative risk, 0.74; 95\% confidence interval, 0.17-3.2). One of the sepsis cases in the loL group resulted in neonatal death because of asphyxia. There were no significant differences in secondary outcomes.

\section{CONCLUSIONS}

The risk of neonatal sepsis after PPROM near term is low. Induction of labor does not reduce this risk. 


\section{BACKGROUND}

Preterm prelabor rupture of membranes (PPROM) is associated with neonatal morbidity and mortality as well as maternal morbidity. ${ }^{1,9,19,145}$ In international guidelines, no clear recommendation is given on the management of PPROM between 34 and 37 weeks. . $^{5,15}, 16$ A recent Cochrane review on the management of PPROM prior to 37 weeks demonstrated insufficient evidence for the management of PPROM in clinical practice. ${ }^{19}$

Given this lack of evidence to justify the induction of labor or expectant management, a randomized controlled trial was performed as the PPROMEXIL (PPROM Expectant Management versus Induction of Labor) trial. ${ }^{89}$ In this trial, we tested the hypothesis that induction of labor (IoL) would reduce the incidence of neonatal sepsis.

In the PPROMEXIL trial, the incidence of neonatal sepsis in the expectant group was $4.1 \%$, which is lower than the expected $7.5 \%$, and the risk of neonatal sepsis was not decreased by induction of labor (2.6\% vs $4.1 \%$; relative risk [RR], $0.64 ; 95 \%$ confidence interval [CI], $0.25-1.6)$. In contrast, in the loL group, the risk of neonatal hypoglycemia and hyperbilirubinemia was increased $(\mathrm{RR}, 2.2 ; 95 \% \mathrm{Cl}, 1.4-3.4$, and $\mathrm{RR}, 1.5 ; 95 \% \mathrm{Cl}, 1.1-1.9$, respectively). Because of this lack of power, there remained equipoise on the subject after the completion of our PPROMEXIL trial.

In view of this equipoise and in view of uncertainty of the continuation of the other large ongoing trial on the subject at that time, Preterm Prelabour Rupture of Membranes Close to Term Trial(PPROMT), ${ }^{128}$ which was dependent on funding, we decided to set up a new trial called PPROMEXIL-2, with a similar design as our PPROMEXIL study, aiming to randomize an additional 200 women. We planned to combine the results of the PPROMEXIL trials with the results of the possible prematurely terminated PPROMT trial into an individual patient data meta-analysis, which would then reach the planned power calculation of the PPROMT trial. The decision to start PPROMEXIL-2 was made after the completion and analysis of the results of PPROMEXIL, and it should therefore be considered as an independent trial.

\section{MATERIALS AND METHODS}

We performed a nationwide randomized controlled trial in The Netherlands between December 2009 until January 2011. The methods of this trial have been described earlier extensively by van der Ham et al. ${ }^{27,89}$ The PPROMEXIL-2 trial was a randomized controlled trial that ran in 60 academic and nonacademic hospitals in The Netherlands. For the PPROMEXIL-2 trial, no changes were made in this trial protocol or in the outcome measures. This trial was registered in the ISRCTN register: ISRCTN05689407 (http://www. 


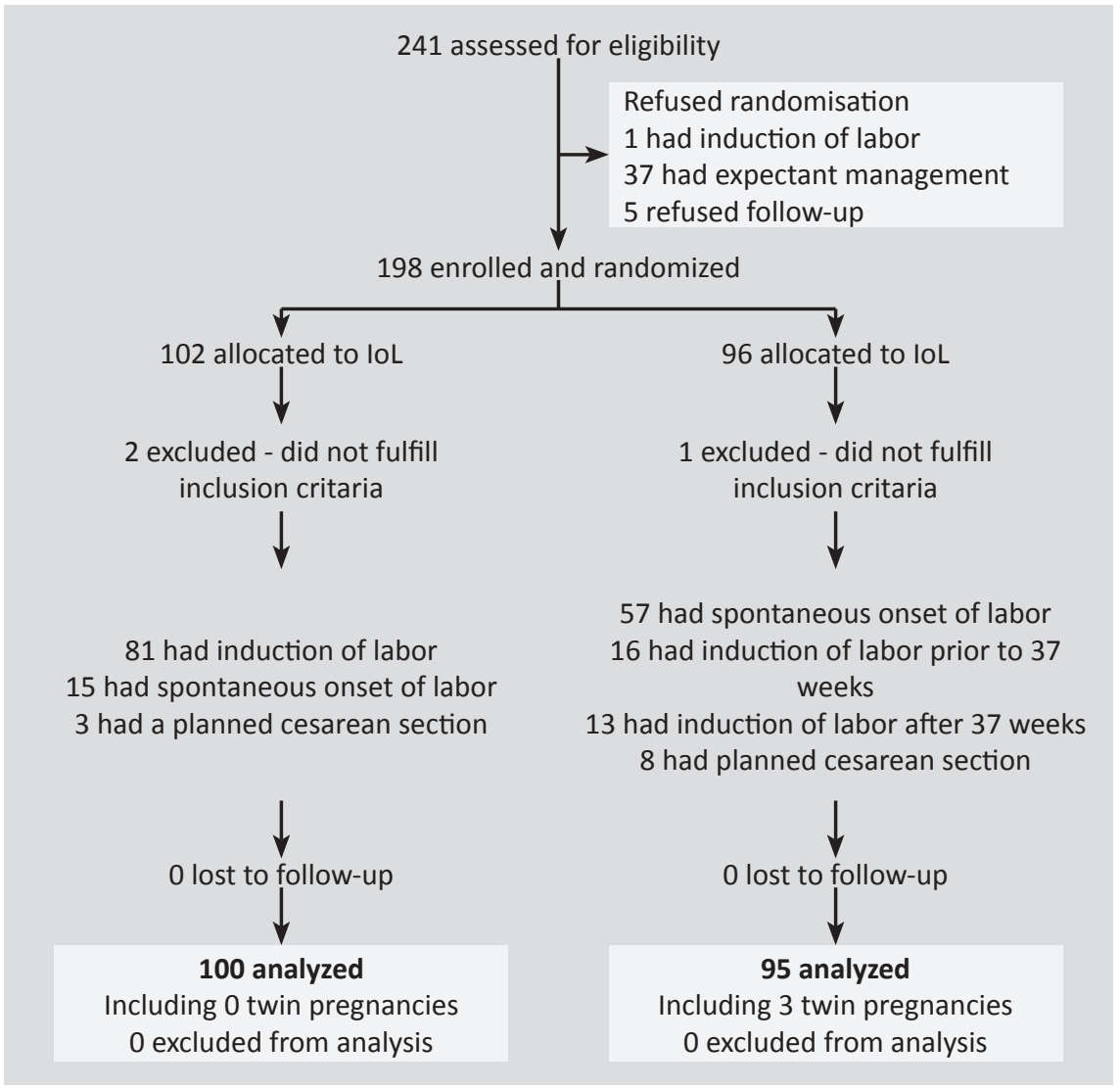

Figure 7.1 | Trial profile

controlledtrials.com/ISRCTN05689407/ppromexil).

The PPROMEXIL-2 study was approved by the Medical Ethics Committee of the Maastricht University Medical Center as an amendment of the PPROMEXIL trial (MEC 05-240). Women with a singleton or twin pregnancy were eligible for the PPROMEXIL trial when they were not in labor 24 hours after PPROM between 34 and 37 weeks of gestational age. PPROM had to be diagnosed after $26^{+0}$ weeks. Women with a monochorionic multiple pregnancy, nonreassuring cardiotocogram, meconium stained amniotic fluid, major fetal anomalies, HELLP (hemolysis, elevated liver enzymes, and low platelets) syndrome, or severe preeclampsia and signs of intrauterine infections were not eligible.

Randomization was performed in a password-protected, web-based database in a 1:1 for immediate delivery ( $\mathrm{loL}$ ) versus expectant management (EM). If women were allocated to loL, labor was induced within 24 hours after randomization. IoL was performed accord- 
ing to the Dutch national guidelines. ${ }^{130}$ If a cesarean section was indicated (for example, in the case of a child in breech position), this was done as soon as feasible after randomization. Women allocated to EM were monitored according to a standard local protocol, until delivery started spontaneously. If a participant reached $37^{+0}$ weeks' gestational age (GA), labor was induced. Labor was induced prior to $37^{+0}$ weeks of gestation when there were clinical signs of infection or on another neonatal or maternal indication that justified induction of labor. Data were collected by research staff in a web-based, passwordprotected database. The Dutch guidelines on PPROM give no clear recommendation on the use of antibiotics prior to labor. Therefore, antibiotics were administered according to local protocol. In pregnancies with PPROM prior to 34 weeks' gestation, corticosteroids were given for fetal pulmonary maturation. Administration of tocolytics was dependent on the local protocol.

\section{OUTCOME MEASURES}

The primary outcome was neonatal sepsis, defined as a positive blood culture taken at birth (not Staphylococcus epidermidis) or within 72 hours 2 or more symptoms of infection (apnea, temperature instability, lethargy, feeding, intolerance, respiratory distress, hemodynamic instability) plus 1 of the following 3 items: (1) positive blood culture (culture-proven sepsis); (b) C-reactive protein greater than 20 (suspicion sepsis); or (3) positive surface cultures of a known virulent pathogen (suspicion of sepsis). When the local investigator classified a case as sepsis or when criteria for sepsis were registered in the database, the case was judged by an independent panel of pediatricians (A.L.M.M., R.M.J.M.) who were not aware of the allocation of randomization. After the relevant data were presented to the panel, they adjudicated between neonatal sepsis (proven or suspected sepsis) or no sepsis. Secondary neonatal outcome measures were respiratory distress syndrome, asphyxia, hypoglycemia, hyperbilirubinemia, total length of hospital stay and admission, and length of stay on the neonatal intensive care unit (NICU) and perinatal death. Maternal outcome measures were antepartum hemorrhage, signs of (histological or clinical) chorioamnionitis, total length of hospital stay, and admission to the intensive care unit. Finally, we recorded mode of delivery and need for anesthesia.

No changes to trial outcomes were made after the trial commenced.

\section{STASTICAL ANALYSIS AND META-ANALYSIS}

Within a well-organized nationwide Dutch research consortium, it seemed feasible to recruit 200 patients within approximately 1 year. These 200 patients combined with the 536 patients of the PPROMEXIL trial ${ }^{89}$ and the estimated number of included patients at the end of 2010 for the PPROMT trial ${ }^{128}$ would provide the power calculation as calculated 


\begin{tabular}{|c|c|c|}
\hline Characteristic $^{a}$ & IoL $(n=100)$ & $\operatorname{EM}(n=95)$ \\
\hline Maternal age (range) $[ \pm S D], y$ & $30.5(19.4-43.6)[ \pm 5.3]$ & $29.4(19.2-41.8)[ \pm 5.0]$ \\
\hline Number nulliparous, n (range) (\%) & $48(0-6)(48 \%)$ & $49(0-4)(52 \%)$ \\
\hline Twin pregnancy, n (\%) & $0(0 \%)$ & \\
\hline \multicolumn{3}{|l|}{ Ethnic origin } \\
\hline White, $n$ (\%) & $78(78 \%)$ & $67(71 \%)$ \\
\hline Other etnic origin, $n(\%)$ & $15(15 \%)$ & $18(19 \%)$ \\
\hline Unknown, n (\%) & $7(7.0 \%)$ & $10(11 \%)$ \\
\hline \multicolumn{3}{|l|}{ Education } \\
\hline Primary school (4 to 12 y), $n(\%)^{b}$ & $0(0 \%)$ & $2(4.0 \%)$ \\
\hline Secondary school (12-18 y), $n(\%)^{b}$ & $9(17 \%)$ & $3(6.0 \%)$ \\
\hline Lower professional school, $n(\%)^{b}$ & $5(9.3 \%)$ & $6(12 \%)$ \\
\hline Medium professional school, $n(\%)^{b}$ & $20(37 \%)$ & $23(46 \%)$ \\
\hline Higher professional school, $n(\%)^{b}$ & $19(35 \%)$ & $11(22 \%)$ \\
\hline University, $n(\%)^{b}$ & $1(1.9 \%)$ & $5(10 \%)$ \\
\hline Maternal smoking, n (\%) & $25(27 \%)$ & $25(27 \%)$ \\
\hline $\begin{array}{l}\text { Antenatal administration of } \\
\text { corticosteroids, } \mathrm{n}(\%)\end{array}$ & $20(22 \%)$ & $13(16 \%)$ \\
\hline \multicolumn{3}{|l|}{ Body mass index } \\
\hline At booking (range) $[ \pm S D], \mathrm{kg} / \mathrm{m}^{2 c}$ & $26.2(16.5-53.3)[ \pm 6.6]$ & $25.0(15.8-46.3)[ \pm 6.4]$ \\
\hline At study entry (range) $[ \pm S D], \mathrm{kg} / \mathrm{m}^{2 c}$ & $30.1(17.8-56.2)[ \pm 8.1]$ & $29.6(20.8-46.3)[ \pm 5.6]$ \\
\hline \multicolumn{3}{|l|}{ Diagnostic test for ruptur of membranes ${ }^{d}$} \\
\hline Positive history, $n$ (\%) & $67(70 \%)$ & $71(76 \%)$ \\
\hline Positive ferning, $n$ (\%) & $48(79 \%)$ & $34(67 \%)$ \\
\hline Positive $\mathrm{pH}$ test, $n$ (\%) & $2(7.7 \%)$ & $2(7.7 \%)$ \\
\hline Positive PAMG-1 test, $n(\%)$ & $11(32 \%)$ & $19(50 \%)$ \\
\hline $\begin{array}{l}\text { Decrease amniotic fluid on ultrasound, } \\
n(\%)\end{array}$ & $53(76 \%)$ & $52(70 \%)$ \\
\hline
\end{tabular}

by the investigators of the PPROMT trial (1812 women). Therefore, no separate power calculation was done for this trial.

Data were analyzed on an intention-to-treat basis. The RRs, absolute risk reduction, mean difference (MD), and 95\% Cls were calculated for the relevant outcome measures. $\mathrm{P}<0.05$ was considered to indicate statistical significance. Statistical analyses were performed using SPSS Statistics (version 17.0; SPSS Inc, Chicago, IL).

We further updated a recent Cochrane review ${ }^{19}$ on the subject for sepsis (overall), culture proven neonatal sepsis, respiratory distress syndrome (RDS), and the cesarean section rate as we did after the PPROMEXIL trial ${ }^{89}$ with the data from the PPROMEXIL trial and the 


\begin{tabular}{|c|c|c|}
\hline Characteristic $^{\mathrm{a}}$ (continued) & IoL $(n=100)$ & $\operatorname{EM}(n=95)$ \\
\hline \multicolumn{3}{|l|}{ Gestational age at PPROM } \\
\hline$<34 w k s, n(\%)$ & $20(20 \%)$ & $13(14 \%)$ \\
\hline $34^{+0}$ to $34^{+6} w k s, n(\%)$ & $11(11 \%)$ & $16(17 \%)$ \\
\hline $35^{+0}$ to $35^{+6} w k s, n(\%)$ & $29(29 \%)$ & $28(30 \%)$ \\
\hline $36^{+0}$ to $36^{+6} w k s, n(\%)$ & $40(40 \%)$ & $37(39 \%)$ \\
\hline $\begin{array}{l}\text { Gestational age at PPROM, median } \\
{[\text { IQR], d }}\end{array}$ & 249 [240-254] & 249 [241-253] \\
\hline $\begin{array}{l}\text { Gestational age at randomization, } \\
\text { median }[\mathrm{IQR}], \mathrm{d}\end{array}$ & $251[242-255]$ & $251[243-255]$ \\
\hline \multicolumn{3}{|l|}{ Fetal position at data entry } \\
\hline Cephalic, $n$ (\%) & 96 (96\%) & $87(92 \%)$ \\
\hline Breech, $n$ (\%) & $4(4.0 \%)$ & $8(8.4 \%)$ \\
\hline $\begin{array}{l}\text { Maternal temperature at inclusion, } \\
\text { mean }[ \pm S D]{ }^{\circ} \mathrm{C}\end{array}$ & $36.8[ \pm 0.44]$ & $36.8[ \pm 0.44]$ \\
\hline \multicolumn{3}{|c|}{$\begin{array}{l}\text { IQR, interquartile range; PAMG-1, placental alpha macroglobulin-1; PPROM, preterm prelabor rupture } \\
\text { of membranes. } \\
\text { a Percentages given are related to available data per characteristic and may differ from total number } \\
\text { of patients; } \\
\text { b Percentages are given as part of known educational level; } \\
\text { c Outcome characteristic with more than } 5 \% \text { missing data; ethnic origin: data available from } 178 \text { cases } \\
\text { ( } 91 \%) \text {; education: data available from } 104 \text { cases ( } 53 \%) \text {; maternal smoking: data available from } 184 \text { ca- } \\
\text { ses ( } 94 \%) \text {; body mass index at booking: data available from } 161 \text { cases ( } 84 \%) \text {; body mass index at start } \\
\text { study available from } 84 \text { cases ( } 44 \%) \text {; antenatal administration of corticosteroids: data available from } \\
173 \text { cases ( } 89 \%) \text {; maternal temperature at inclusion: data available from } 172 \text { cases }(90 \%) ; \\
\text { d Sum of tests exceeds } 100 \% \text { because more than } 1 \text { test could be applied on the same patient; percen- } \\
\text { tages are given as part of applied tests. Data on positive history were available from } 190 \text { of } 195 \text { cases } \\
\text { ( } 97 \%) \text {. Ferning was done in } 112 \text { cases, pH test was done in } 52 \text { cases, PAMG-1 test was done in } 72 \\
\text { cases, and ultrasound was done in } 146 \text { cases; } \\
\text { e In one woman, the term at rupture of membranes was unknown. }\end{array}$} \\
\hline
\end{tabular}

Table 7.1 | Baseline characteristics

current PPROMEXIL-2 trial, using Review Manager Software version 5.1.131

\section{RESULTS}

From December 2009 until January 2011, a total of 241 women were asked to participate in the trial, of which 198 women (82\%) gave informed consent. Of these women, 3 had to be excluded because they had been randomized at a gestational age longer than $36^{+6}$ weeks. The remaining 195 women were eligible for analysis. A total of 100 women were randomized to induction of labor (loL group) and 95 to expectant management (EM 
group). Figure 7.1 outlines the study profile. Baseline characteristics are shown in table 7.1. The median gestational age at randomization was 251 days. Thirty three women (17\%) had PPROM prior to 34 weeks' GA. Table 7.2 shows data on pregnancy outcome and mode of delivery. Women in the loL group delivered on average 3.5 days earlier (95\% $\mathrm{Cl}, 1.8-5.2$ days) than women in the EM group. Women in the EM group stayed on average 4.4 days longer in the hospital ( $95 \% \mathrm{Cl}, 2.2-6.7$ days).

The mode of delivery was not statistically significant different. There were fewer cesarean sections in the loL group (13 [13\%] vs 22 [22\%]; RR, 0.58; 95\% Cl, 0.31-1.08; $\mathrm{P}=0.081$ ). This difference was partly because of the higher number of planned cesarean sections in the EM-group (3 vs 8), which was known at baseline and could not be due to randomization.

Antibiotics during admission and during labor were administered equally.

There were no differences in the rates of epidural and/or spinal analgesia.

\section{NEONATAL SEPSIS}

Neonatal sepsis was seen in 3 neonates (3.0\%) in the loL group versus four $(4.1 \%)$ in the EM group (RR, 0.74; 95\% Cl, 0.17-3.2) (Table 7.3). One neonate in the loL group who had a proven sepsis died 48 hours postpartum because of the complications of a severe asphyxia and anemia. During labor, fetal blood sampling was performed because of a suboptimal cardiotocography. This procedure resulted in heavy blood loss, after which an emergency cesarean section was performed. An asphyctic male neonate (arterial pH 6.98 $\mathrm{mmol} / \mathrm{L}$ and Apgar score 0/0) was born and was transferred to an NICU-center in which multi-organ failure occurred with a Sarnat stage 3 asphyxia and positive blood cultures for group B-Streptococcus. The child died 48 hours postpartum. This case was reported to the Medical Ethical Committee of the Maastricht University Medical Center, and it was extensively discussed by our panel of neonatologists (A.L.M.M, R.M.J.M.) as well as by an independent gynecologist. Neonatal death was considered to be related to the severe asphyxia and anemia and not to neonatal sepsis. Induction of labor was not considered to be the cause of this severe adverse event.

\section{OTHER NEONATAL OUTCOMES}

Table 7.3 shows all neonatal outcomes. Neonates born in the loL group stayed 7.4 days in the hospital compared with 6.9 days (MD, $0.52 ; 95 \% \mathrm{Cl}$, -0.5 to 2.3 days) after EM. Neonates in the loL group were equally admitted to the NICU (7 [7.0\%] cases vs 8 [8.2\%] in the EM group; RR, 0.86; $95 \% \mathrm{Cl}, 0.32-2.3$ ). Newborns admitted to the NICU in the IoL group stayed a shorter time than those in the EM group (mean 2.0 vs 7.0 days; MD, -5.0; $95 \% \mathrm{Cl},-9.0$ to -1.0$)$. Respiratory distress syndrome was seen in 6 newborns in the loL 
group (6.0\%) versus 5 in the EM group (5.1\%) (RR, 1.2; 95\% Cl, 0.37-3.7). Hypoglycemia (8 [8.1\%] vs 8 [8.2\%]; RR, 0.99; 95\% Cl, $0.39-2.5$ ) and hyperbilirubinemia (20 [20\%] vs 21 [21\%]; RR, 0.95; 95\% Cl, 0.55-1.6) were seen equally in both groups. For other neonatal outcome measures, there were also no significant differences between both groups.

\section{MATERNAL OUTCOMES}

Table 7.4 shows all maternal outcomes. Clinical chorioamnionitis was not seen in the loL group and in 4 women in the EM group (4.3\%) ( $P=0.038)$. The incidence of histological chorioamnionitis was 12 (18\%) versus 18 (31\%), respectively (RR, 0.64; 95\% Cl, 0.33-1.2).

\section{META-ANALYSIS}

In total 1428 neonates could be analyzed from 9 studies for neonatal sepsis, 1090 neonates (6 studies) for culture-proven sepsis, 1428 neonates (9 studies) for RDS, and 1417 women ( 9 studies) for cesarean section rate. As shown in figure 7.2, the risk ratio of all outcome were not statistically different.

\section{COMMENT}

In this PPROMEXIL-2 trial, 195 women with PPROM between 34 and 37 weeks were included and analyzed. We found that induction of labor did not reduce the incidence of neonatal sepsis, nor did it influence the rates of cesarean section and RDS. Because all cases with possible signs for neonatal sepsis were adjudicated by a panel of neonatologists, we believe that we did not miss any case of neonatal sepsis, nor did we overestimate the incidence of neonatal sepsis. Induction of labor did reduce the risk of clinical chorioamnionitis, but we did not find a significant difference in histological chorioamnionitis. Nevertheless, incidences of chorioamnionitis were in the same magnitude as in the PPROMEXIL trial. It has been suggested in the previous studies that chorioamnionitis is related to cerebral palsy. ${ }^{132-135}$ However, we believe that this association cannot be extrapolated to our population because these studies were reporting on very preterm infants. Because the incidence of cerebral palsy in the near term population is very low, we do not believe that this association justifies induction of labor in women with late preterm PROM.

In contrast to the PPROMEXIL trial, ${ }^{89}$ we found no difference in the incidence of hypoglycemia and hyperbilirubinemia between both groups.

As shown in the meta-analysis based on more than 1400 neonates, expectant management seems to be a safe strategy with respect to neonatal sepsis, RDS, and cesarean section rates. 


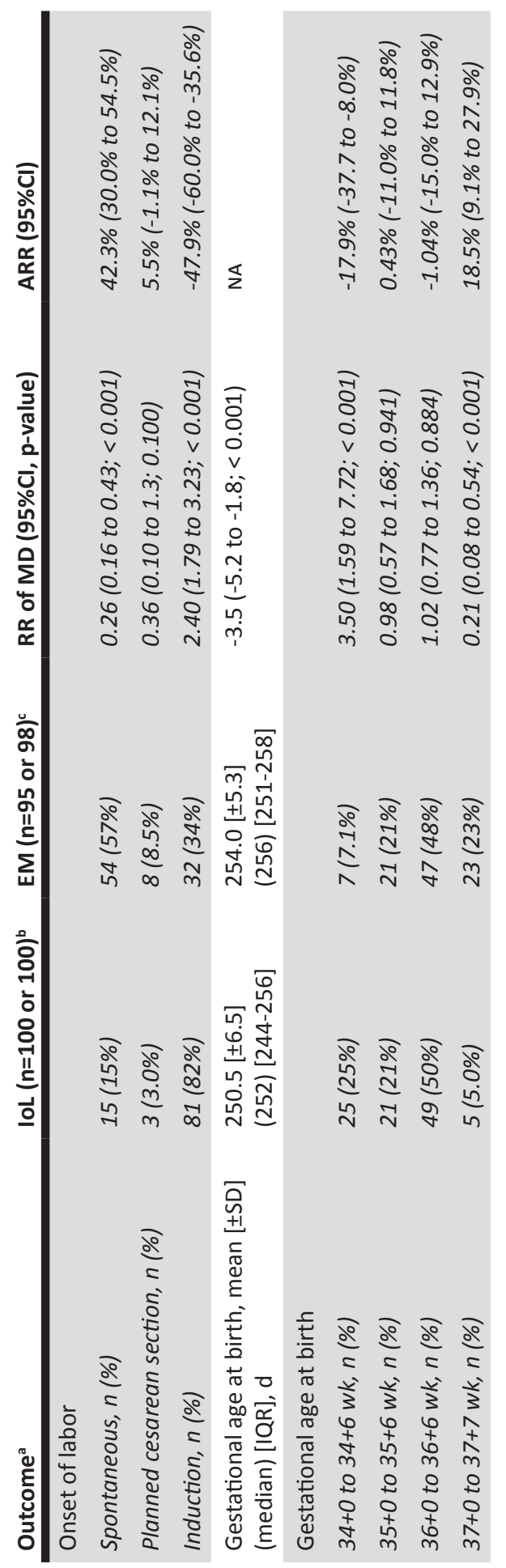




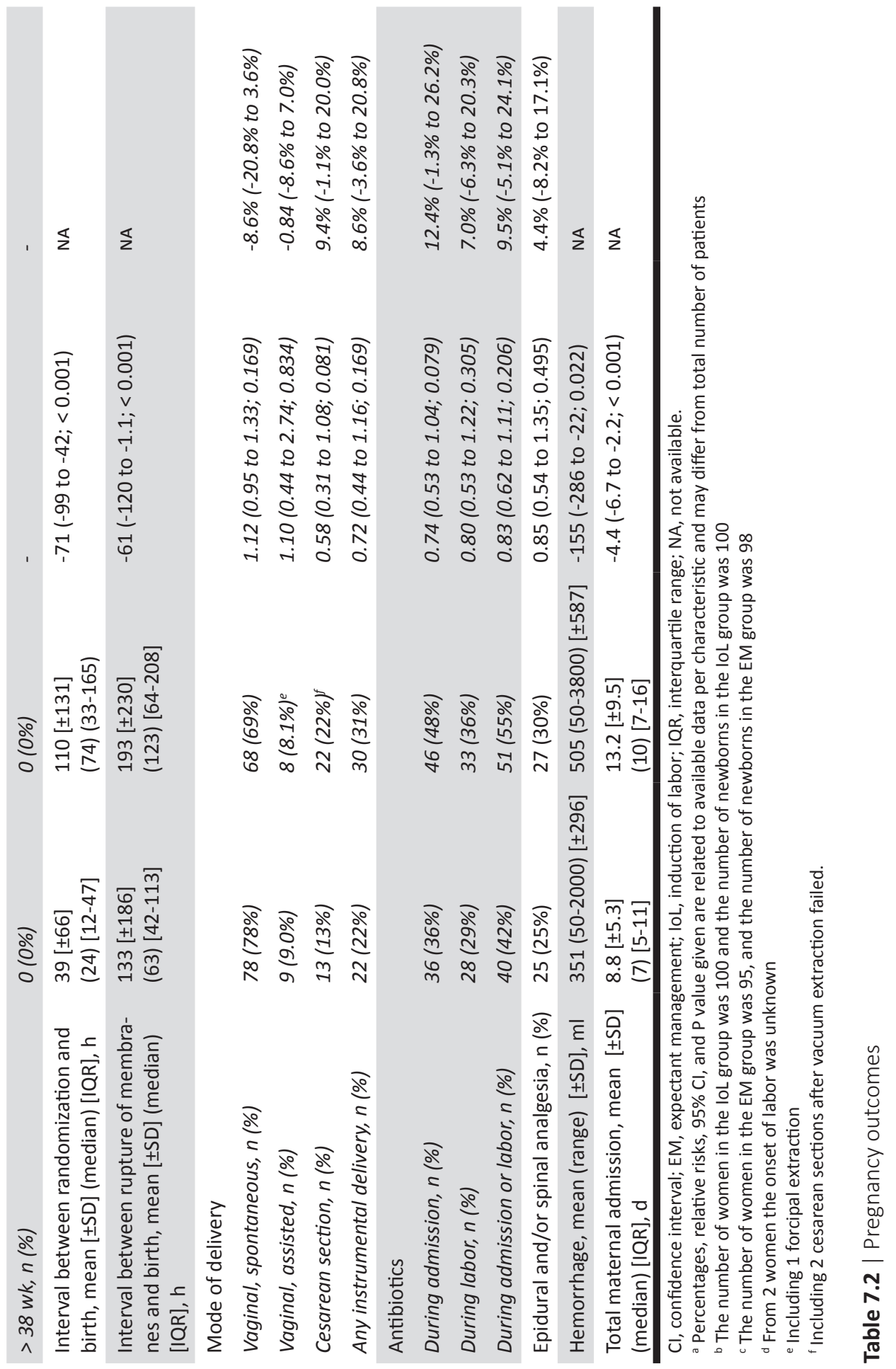




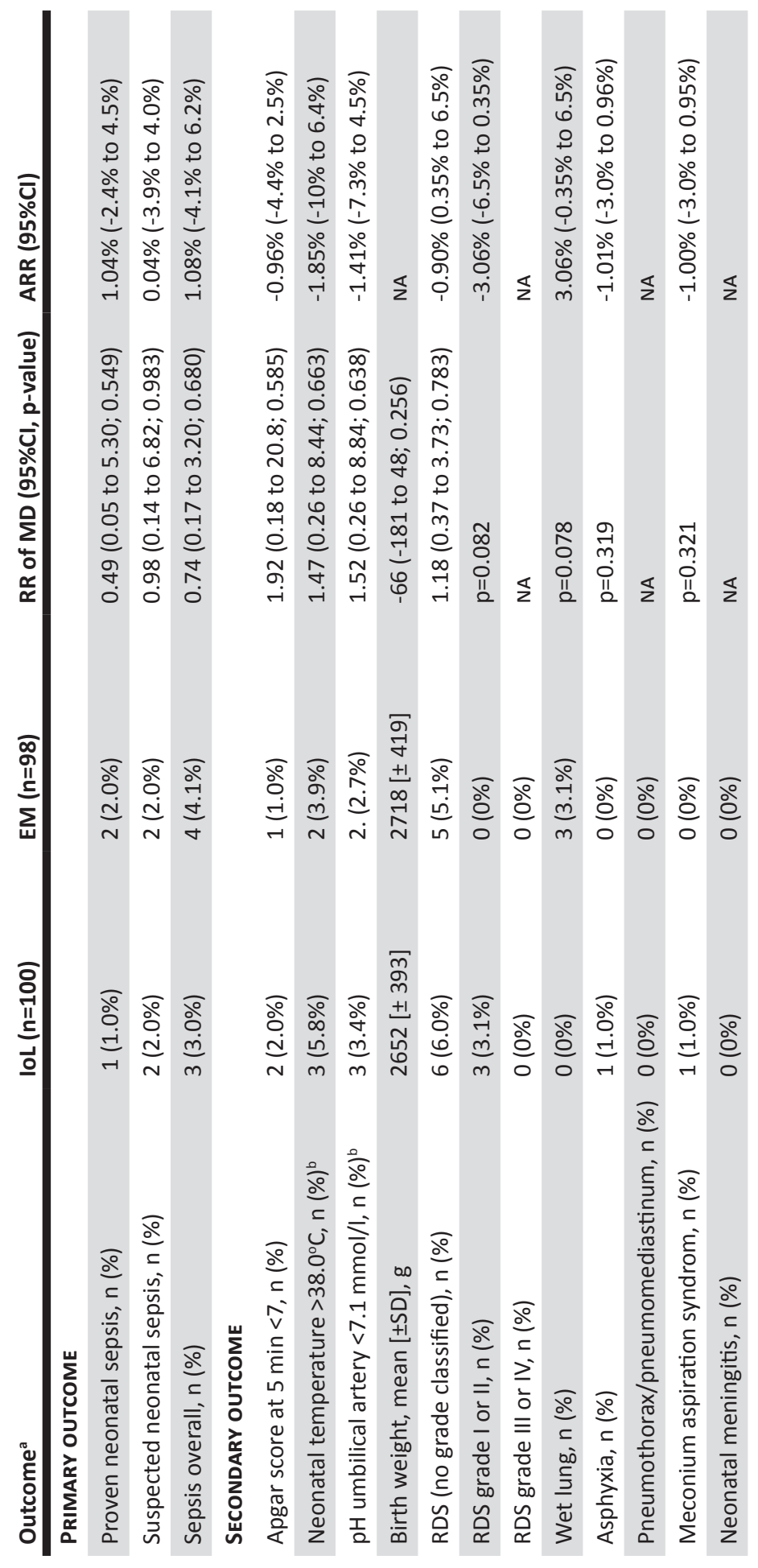




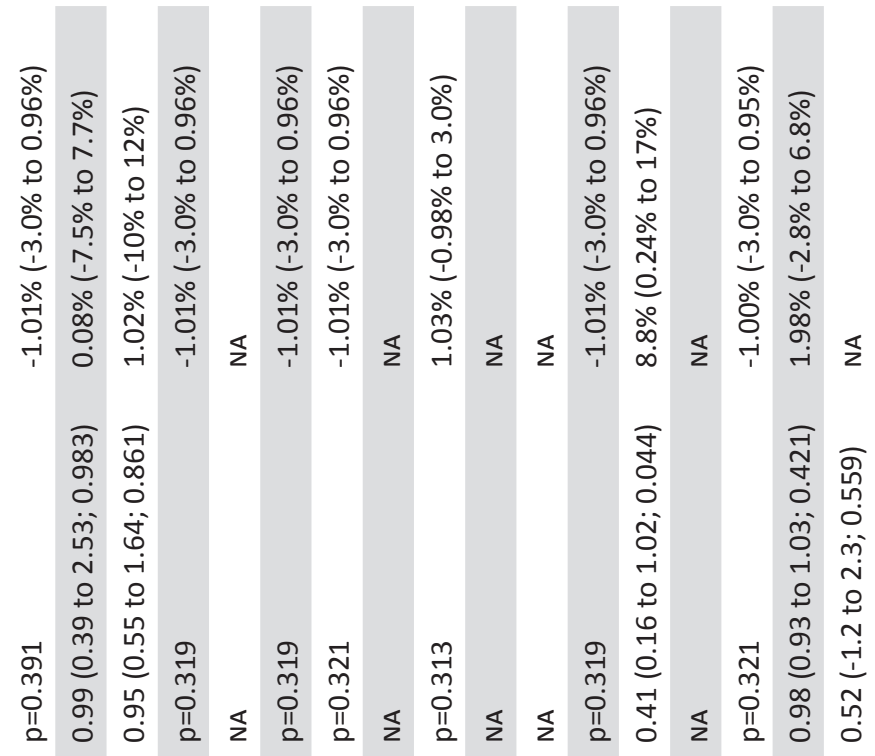

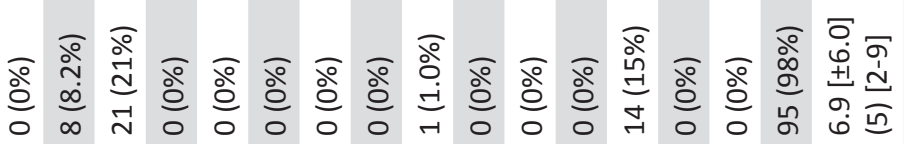

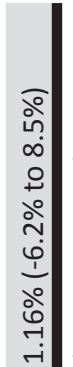

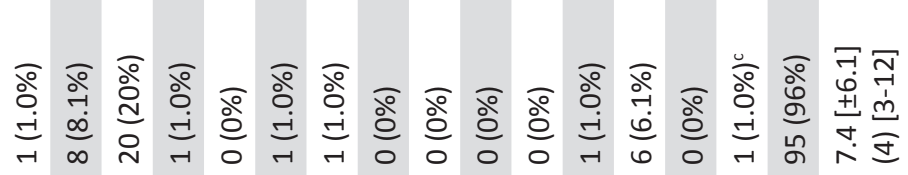




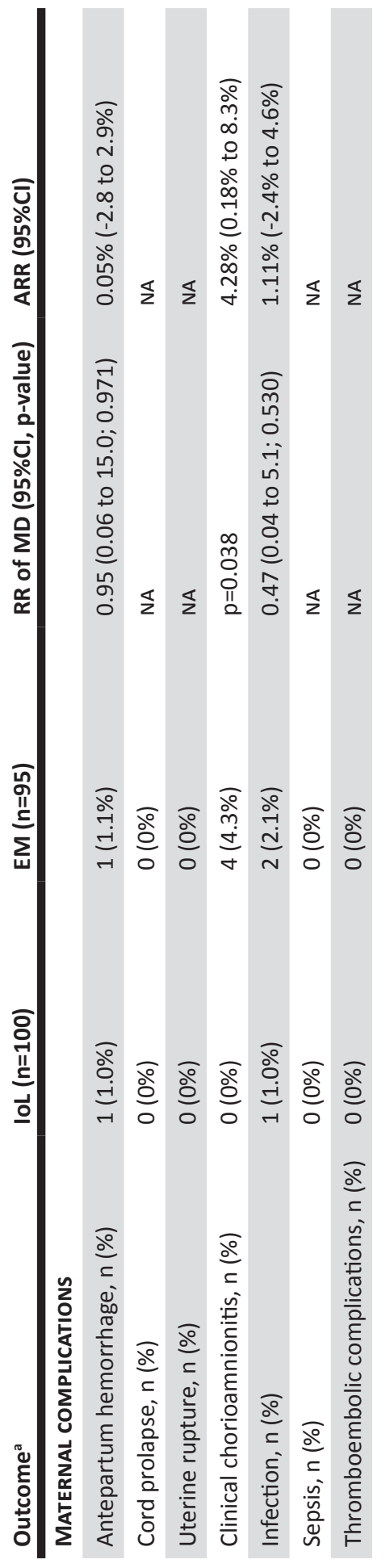



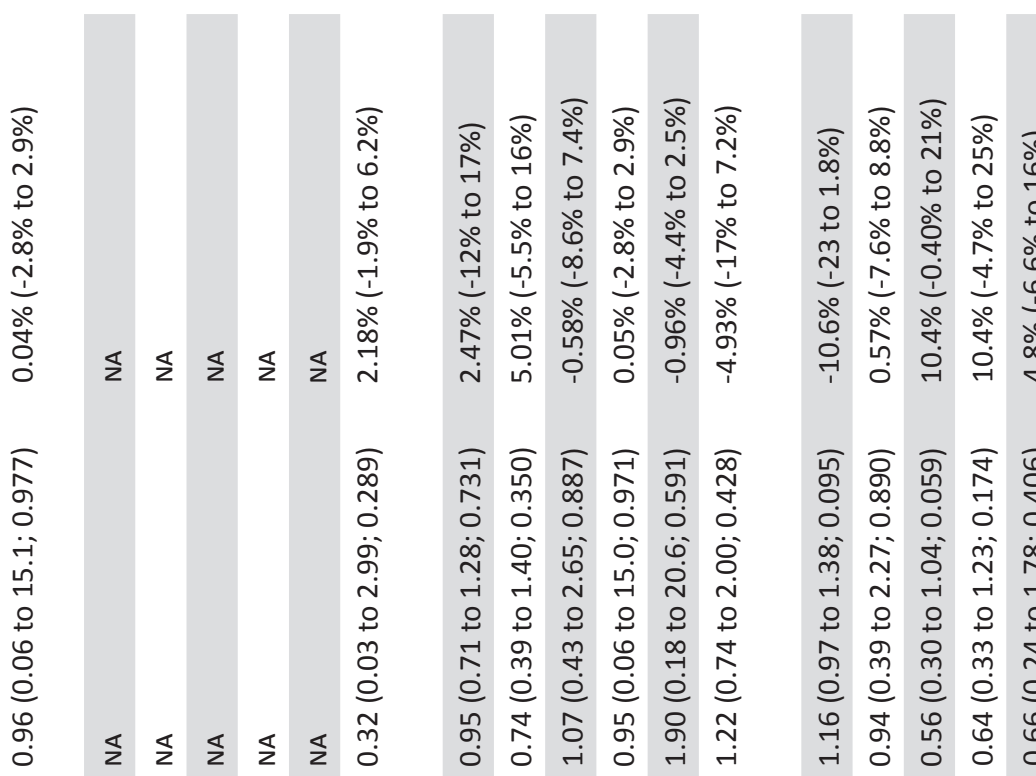

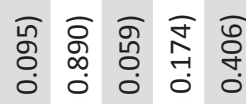
$\dot{\infty} \ddot{\sim} \dot{\sim} \ddot{\sim} \dot{\sim}$

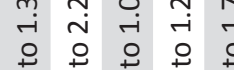
ศิ ก) ำ m 응 응 元
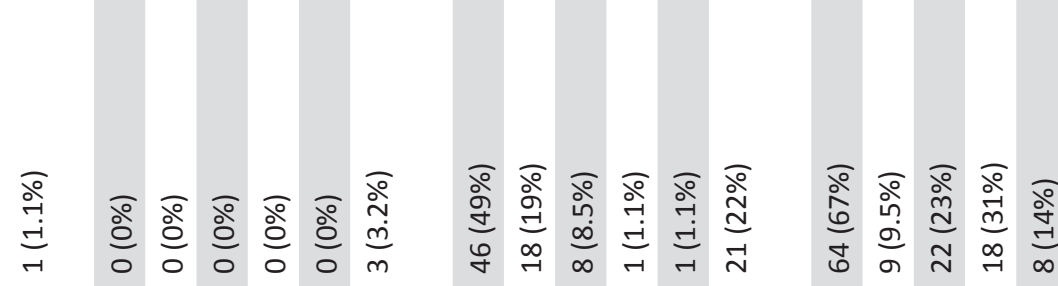

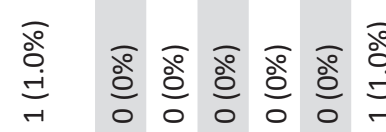
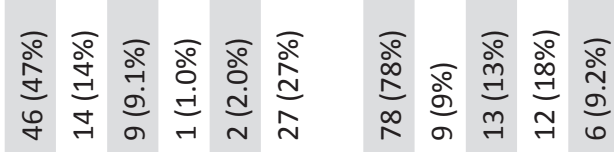

in $\frac{\pi}{\pi}$ 选

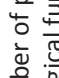
है 음 吾 웅 든

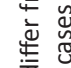
तั है $\frac{\varepsilon}{0}$

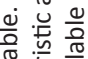

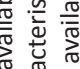
읃 空 党 这高:

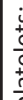
过 $\frac{\pi}{2} \frac{0}{0}$ 그 $\frac{\bar{\pi}}{\mathrm{T}}$

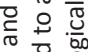
i 엉 으 范

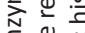
님 竞

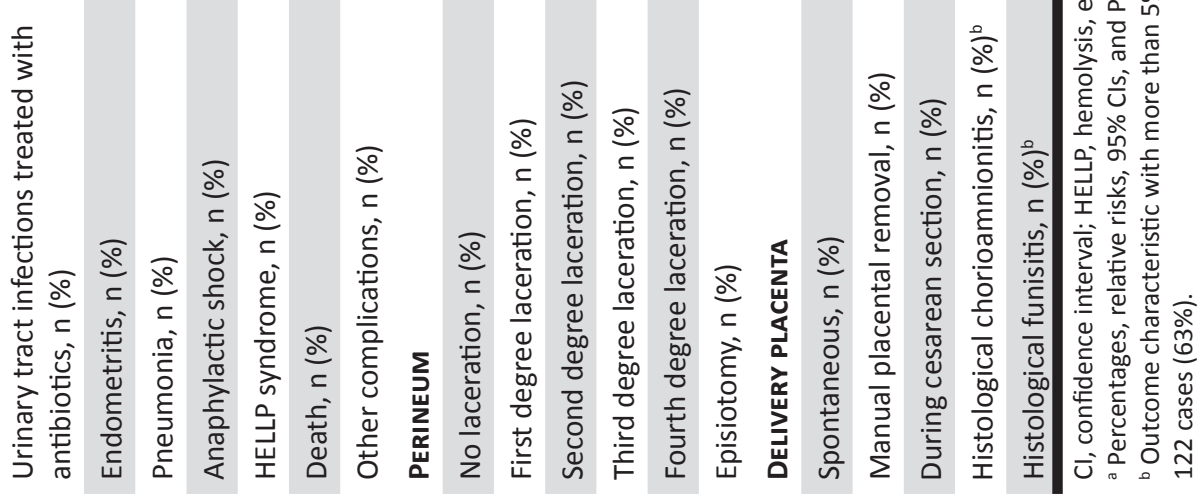

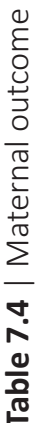




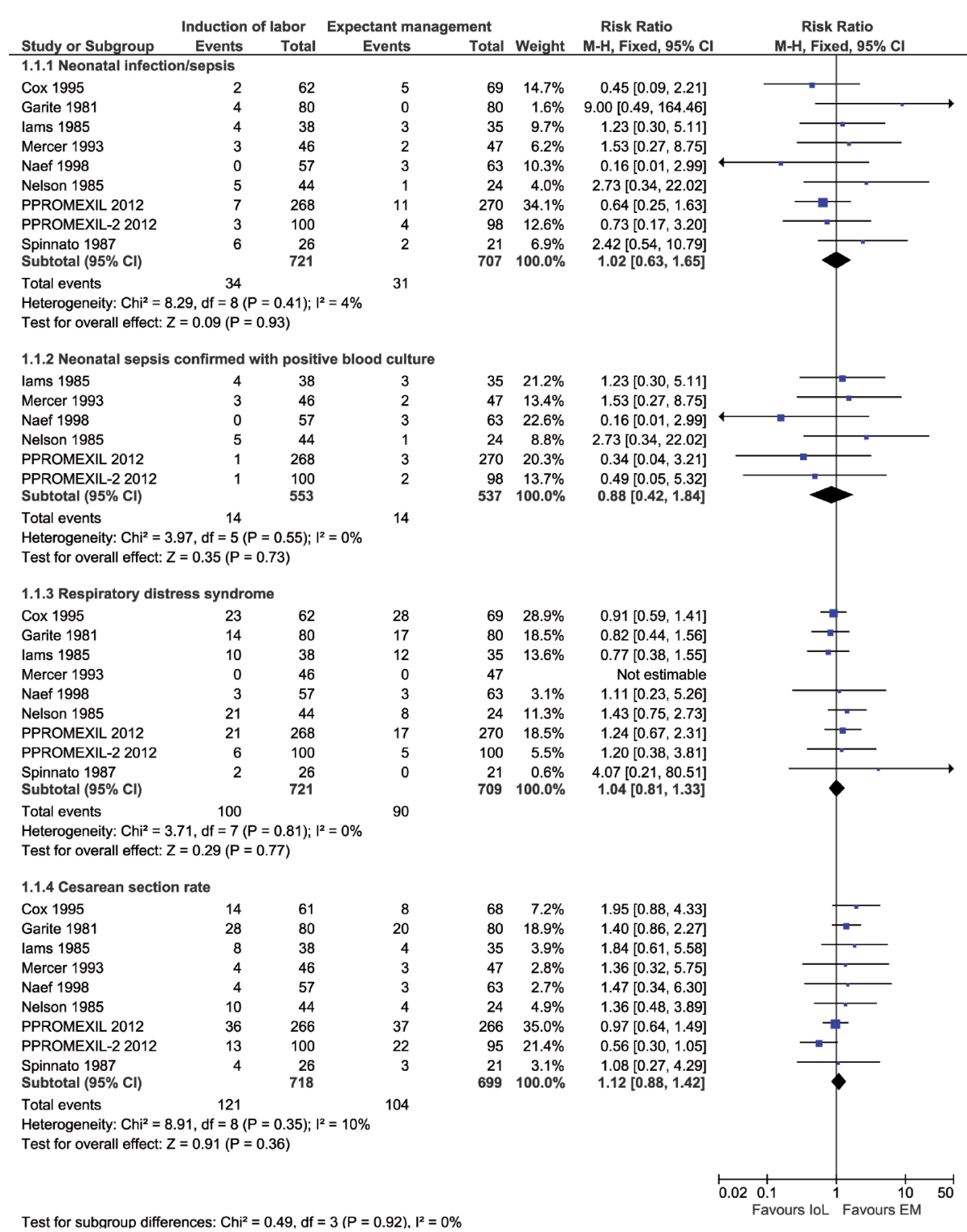

Figure 7.2 | Meta analysis

This trial has its limitations. As mentioned in the introductory text, the design of the study was approved and registered after we finished the PPROMEXIL trial and should therefore be considered as a separate trial. Because of the remaining equipoise at that moment and major funding problems of the ongoing PPROMT trial, ${ }^{128}$ this smaller additional trial was executed to improve the number of inclusion to perform and individual patient data 
meta-analysis (IPD-MA) with data of PPROMT and both PPROMEXIL trials. Recruitment of an additional 200 women within a 12 month period seemed feasible. However, near the closing of the recruitment of the patients for the PPROMEXIL-2 trial, the investigators of the PPROMT trial gained extra funding to complete their estimated inclusions (1812 women). The results as presented in the current trial should be interpreted with some caution because of the fact that no proper power calculation was done.

As in the PPROMEXIL trial ${ }^{89}$ during which we observed lower-than-expected sepsis rates ( $2.6 \%$ in the IoL group vs $4.1 \%$ in the EM group), the incidences of sepsis in the PPROMEXIL-2 trial were low (3.0\% vs 4.1\%, respectively). The liberal use of antibiotic therapy before or during labor (overall $47 \%$ received antibiotics) might have contributed to a lower incidence compared with the other trials in which antibiotics were not administered prophylactically. ${ }^{20-26}$ Improvements in the health care system over the last decades may have contributed to a reduction of the incidence of neonatal sepsis. Expectant management prolonged gestation with 4 days, and this rather small difference, which was in line with the PPROMEXIL trial, might partly be due to the fact that the median gestational age at rupture of membranes was $35^{+4}$ weeks and the median gestational age at randomization was $35^{+6}$ weeks. The overrepresentation of women with gestational age longer than 35 weeks can be caused by the fact that women between 34 and 35 weeks of gestation more often refused to participate (mean gestational age at PPROM in the nonrandomized group was $34^{+6}$ weeks). Furthermore, the hesitation of clinicians to induce labor before 35 weeks of gestation, which was not recommended in the Dutch guideline prior to the start of the PPROMEXIL trial, ${ }^{5}$ may also have influenced this outcome. If we combine the results of both PPROMEXIL trials for neonatal sepsis, we find a relative risk of 0.66 (95\% $\mathrm{Cl}, 0.30-1.5)$, and the absolute risk reduction is $1.4 \%(95 \% \mathrm{Cl},-4.0 \%$ to $1.3 \%)$. The number needed to treat with the current combined result of the PPROMEXIL trials is 71 for 1 case of neonatal sepsis. Even if a larger trial like the current ongoing PPROMT trial ${ }^{128}$ or a metaanalysis with independent patient data (IPD-MA) of the current PPROMEXIL trials with the PPROMT trial will find a significant difference, its clinical relevance might be debated. In view of our recently completed PPROMEXIL and PPROMEXIL-2 studies and in view of the ongoing Australian initiated PPROMT study, one could question whether we could plan the generation of evidence more efficiently from a global perspective. Although we are in close contact with the PPROMT investigators, prospective trial registration at the moment that trials are planned would have been helpful. One could have collaborative execution of the trials under the umbrella of a prospective individual patient data metaanalysis, leaving the decision when to stop studies in such a collaborative to a Data Safety Monitoring Board overseeing all the trials. Until such scenarios have become reality, we believe that planning similar trials in different countries with a post-hoc meta-analysis of 
data is the best alternative.

In conclusion, this current trial expanded the amount of evidence on the management of near-term PPROM with an additional 195 women. Still, the incidence of neonatal sepsis is low after these pregnancies, and this rate is not reduced by induction of labor. Induction of labor does not increase the risk of any other adverse neonatal or maternal outcome. To this date, the PPROMEXIL trials and updated meta-analysis provide in our opinion enough evidence to prefer expectant management in women with near-term PPROM. 



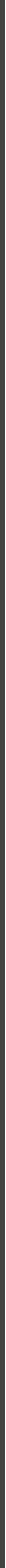




\section{CHAPTER}

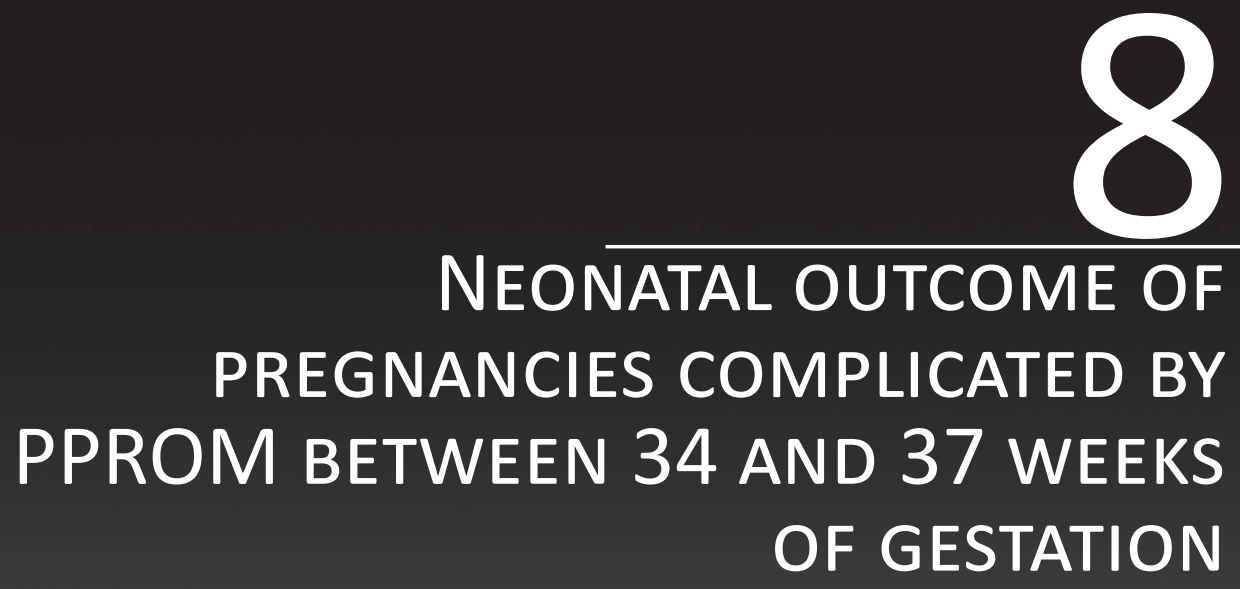

David P van der Ham, Anita CJ Ravelli, Jelle M Schaaf, Christine Willekes, Antonius LM Mulder, Johannes J van Beek, Ben Willem J Mol, Jan G Nijhuis

Submitted 


\section{ABStraCt}

\section{Objective}

The optimal management of late preterm prelabor rupture of membranes (PPROM) is still uncertain. We therefore retrospectively studied neonatal outcome in late preterm PROM (34 to 37 weeks).

\section{Methods}

Data were obtained from the national Perinatal Registry Foundation of the Netherlands from 1999 to 2006. We included all singleton pregnancies complicated by PPROM from $34^{+0}$ until $37^{+0}$ weeks gestational age with an interval between rupture of membranes (ROM) and birth of at least 24 hours. Spontaneous preterm deliveries (34-37 weeks) served as controls. Pregnancies with congenital anomalies, PE/HELLP and/or growth restriction were excluded. The outcome of pregnancies with and without PPROM were compared with respect to neonatal outcome and mode of delivery and stillbirth.

\section{RESULTS}

In total 2,670 women had PPROM and 22,180 had spontaneous delivery without PPROM. Overall $3.2 \%$ of the neonates in the PPROM group versus $2.6 \%$ in controls suffered neonatal sepsis (RR $1.2595 \% \mathrm{Cl} 0.99$ to 1.6 ). The risks of hypoglycemia, hyperbilirubinemia, oxygen therapy, respiratory distress and airway infections were all significantly decreased in the PPROM group. The risk of stillbirth was significantly lower in PROM compared to controls ( 0.1 versus $0.3 \%$ ). Women with PPROM had more often a vaginal instrumental delivery (15\% versus 9\%; RR $1.6(95 \% \mathrm{Cl} 1.5-1.8)$, but less often a Cesarian section ( $7 \%$ versus $10 \%$; RR 0.77 $95 \% \mathrm{Cl} 0.67$ to 0.88$)$.

\section{CONCLUSION}

Prolongation of pregnancy in women with late preterm PROM reduces the risks of prematurity without increasing the risk of stillbirth. Expectant management should therefore be the treatment of choice. 


\section{INTRODUCTION}

Preterm premature rupture of the fetal membranes (PPROM) is associated with an increased incidence of chorioamnionitis and prematurity. It complicates 1 to $5 \%$ of preterm pregnancies, accounting for $30-40 \%$ of all preterm deliveries, ${ }^{30-32}$ and is associated with an increase in fetal and maternal morbidity and mortality. ${ }^{1,2,9,19}$ It is a dilemma for the perinatal team as awaiting spontaneous labor may increase the risk of infectious diseases and stillbirth, whereas induction of labor leads to preterm birth and thus increased neonatal morbidity.

International guidelines have different opinions on the treatment of near term PPROM $^{15,16,147}$ and two recent survey's and a Cochrane review confirm the global lack of consensus and the lack of evidence to guide clinical practice on this issue. ${ }^{17-19}$ In the recent PPROMEXIL-trials, the largest RCTs on the management of PPROM late preterm so far, we concluded that induction of labor did not result in a substantial improvement of the outcome of pregnancy. ${ }^{89,148}$ We are aware of another ongoing randomized controlled trials comparing induction of labor versus expectant management in women with PPROM between 34 and 37 weeks gestational age. ${ }^{149}$

In view of the continuous uncertainty on the optimal management in women with late PPROM, we performed a large retrospective study, using the Dutch nationwide perinatal database (PRN). The purpose of this retrospective study was to analyze neonatal outcome in a larger scale than any RCT can possibly be able to, due to funding limitations, in patients with PPROM between 34 and 37 weeks of gestational age (GA) over a period from 1999-2006 using the national PRN database. ${ }^{150}$

\section{Materials AND MethodS}

\section{DATA SELECTION}

Data were obtained from the national Perinatal Register Foundation of The Netherlands from 1999 up and including 2006. ${ }^{150}$ Since 1999 the PRN combines all delivery record (National Delivery Record - LVR), of both midwives (LVR-1) and obstetricians (LVR-2) with all neonatal admission records from the pediatrician/neonatologist (National Neonatal Register, LNR) into a national perinatal register (PRN). Méray and Tromp et al. have described the technical approach and subsequent validation of the probabilistic linkage of these three population bases registries. ${ }^{151,152}$ Since 1999 the Dutch Perinatal Registry Foundation combines all delivery and neonatal records into a perinatal register (PRN). ${ }^{150}$ The LVR-1 and LVR-2 register have a 96\% national coverage of all births whereas the neonatal register has a $68 \%$ coverage of admissions after birth. This difference is due to the 
fact that registration of perinatal records is mandatory whereas registration of neonatal records is not mandatory for non 3-level centers.

For the present study we selected all pregnancies complicated by late preterm PROM (study group). PROM was defined as an interval of more than 24 hours between rupture of membranes and labor. Women with PPROM between $34^{+0}$ and $36^{+6}$ weeks GA were included in the study. Women who delivered between $34^{+0}$ and $36^{+6}$ weeks GA with a spontaneous onset of labor within 24 hours after PROM served as controls (control group). The date of PPROM had therefore to be known in all women, and women in whom this information was not available were excluded from the analysis

Because of the conservative treatment strategy in The Netherlands the majority of women with PPROM was not induced before 37 weeks of gestation. In a survey among 210 obstetricians and residents (82.4\%), performed before the results of our PPROMEXIL trials were known treated patients expectantly, 11.4\% induced labor and 6.2\% did alter their treatment between patients (unpublished data). This rather conservative treatment strategy makes the Dutch population very suitable for a retrospective analysis of the risks of expectant management in PPROM.

For both groups exclusion criteria were twin pregnancy, HELLP syndrome preeclampsia or pregnancy induced hypertension defined as a diastolic blood pressure $>90 \mathrm{mmHg}$ with or without albuminuria, birth weight below $2.3^{\text {rd }}$ weight percentile or birth weight below $10^{\text {th }}$ weight percentile and suspicion of intrauterine growth restriction, congenital malformations, stillbirth. In the control group additional exclusion criteria were induction of labor and elective cesarean section. Furthermore premature home and outpatients deliveries (LVR-1) were excluded. Patients who delivered in hospitals that did not participated in the LNR registry or for less than 2 years were also excluded.

\section{Outcome}

Maternal baseline characteristics recorded were maternal age at delivery, parity, ethnicity, GA at ROM and GA at delivery. Outcome variables recorded were birth weight, 5 minutes Apgar score $<7$ and mode of delivery.

The primary neonatal outcome was neonatal sepsis defined as a diagnosis of sepsis by the neonatologist in the first admission of the child after birth in the LNR registry. Other poor neonatal outcomes were defined according to a similar study by Langenveld et al. on neonatal outcome of pregnancies complicated by hypertensive disorder between 34 and 37 weeks ${ }^{153}$ and included admission to the neonatal intensive care unit (NICU), neonatal meningitis, metabolic and gastrointestinal morbidity subdivided in hypoglycemia (excluding hypoglycemia due to gestational diabetes and defined as glucose serum plasma level $<2.5 \mathrm{mmol} / \mathrm{l}$ ), hyperbilirubinemia (indirect hyperbilirubinemia defined as above 
the phototherapy level, direct hyperbilirubinemia defined as $>10 \%$ of the total serum bilirubin) and any stage of necrotizing enterocolitis (NEC). ${ }^{154}$ Respiratory problems were subdivided in the need for oxygen therapy more than 24 hours, any grade of infant respiratory distress syndrome (RDS: base on radiographic thorax findings according to Giedeon grade I-IV), respiratory problems other than RDS and bronchopulmonary dysplasia (BPD; defined as more than 28 days oxygen therapy or oxygen therapy after 36 weeks gestational age). Neurologic morbidity was subdivided into intracranial hemorrhage including intraventricular hemorrhage (IVH, defined according to Papile grade I-IV), cerebral ischemia subdivided in any grade of periventricular leucomalacia (PVL, grade I-IV), or cerebral ischemia other than PVL, any stage of hypoxic ischemic encephalopathy (HIE, Sarnat stage 1 till 3) and convulsions. ${ }^{123,155,156}$ and neonatal death. Neonatal death is defined as death after birth within the first week of life. For this study only data of the first admission of the child after birth were used.

\section{STILLBIRTH}

One of the major concerns due to prolonged rupture of membranes in PPROM is the risk of intrauterine and intrapartum death. In the register this is defined as a combined outcome stillbirth. Because the low incidence of intrauterine and intrapartum death, prospective randomized controlled trials have until now not been able to identify this risk. The LNR dataset provides the possibility to estimate these risk. In contrast to the other neonatal outcome this outcome data can be obtained from LVR (delivery) registries only, which covers $96 \%$ of all deliveries in the Netherlands. This more than doubles the size of the database. Because of the importance of this outcome we decided to make a separate selection for stillbirth. We assumed that at a gestational age of 34 weeks all babies were alive. If a stillbirth had occurred before 34 weeks, the women had most likely delivered within a few days after the diagnosis stillbirth. We selected data from the PRN registry, women who delivered before 34 weeks as well as twin pregnancy, any congenital malformation, births under supervision of a midwife only, pre-eclampsia or pregnancy induced hypertension and HELLP syndrome and severe growth restriction $(p<2.3)$ were excluded. We subdivided the women in two groups (ROM $\leq 24$ hours; controls and ROM > 24 hours; PROM). For both groups a Kaplan-Meier survival curve was constructed between 34 and 37 weeks of gestational age. A subgroup analysis was done for small for gestational age (birth weight <p10) and normal birth weight.

\section{STATISTICAL ANALYSIS}

Data were analyzed according to gestational age at PPROM as well as interval between PPROM and delivery. In the first analysis controls who delivered at a certain gestational 
age were compared to women with PPROM at that certain gestational age. In the second analysis we analyzed the outcome in respect of latency by subdividing the interval of PPROM in five subgroup (<24 hours (controls), 24-29 hours, 30-71 hours, 72-119 hours and $>120$ hours of PPROM).

A subgroup analysis was performed for neonatal outcome in relation to the gestational age groups per week (34, 35 and 36 weeks of GA, respectively). For the primary outcome neonatal sepsis a subgroup analysis was not only performed according to gestational age but also within the separate groups and in relation to the interval of PPROM.

Data are presented as N (\%) and mean ( \pm SD). We use Chi-Square to test categorical data and Students T-test or one-way ANOVA (in case of $>2$ variables) for continuous variables. The probability of $\mathrm{p}<0.05$ was considered statistically significant. Data are expressed as odds ratio's (OR) or relative risk (RR) and 95\% confidence interval $(\mathrm{Cl})$. In a multivariable analysis we assessed whether duration of PPROM had impact on neonatal outcome, outcomes like sepsis, with adjusted for parity, maternal age and ethnicity in the total group and stratified for week of gestation. The adjusted outcome are presented as adjusted odds ratio's. All analysis were performed using SAS software (Version 9.2, SAS Institute, Carry NC).

\section{RESULTS}

From January 1st 1999 until December 31st 2006 a total of 1,464,533 women delivered in the Netherlands. After applying the exclusion criteria, as is shown in figure 8.1, we could select 2,670 pregnancies which were complicated by PPROM between $34^{+0}$ and $36^{+6}$ weeks GA. A total of 22,180 women served as controls, as they spontaneously delivered between $34^{+0}$ and $36^{+6}$ weeks GA within 24 hours after PROM. Figure 8.1 shows the selection process of the 24,850 records.

\section{BASELINE CHARACTERISTICS AND MODE OF DELIVERY}

Baseline characteristics and mode of delivery are outlined in table 8.1. Overall the PPROM group were more often nulliparous (70\% versus 59\%, $p=<0.0001)$. In total 2,070 women (78\%) in the PPROM group delivered spontaneously versus 17,947 (81\%) in the control group (RR 0.96; 95\% Cl 0.94 to 0.98), 395 (15\%) had a vaginal operative delivery in the PPROM group versus 2,016 $(9.1 \%$ ) in controls (RR $1.63 ; 95 \% \mathrm{Cl} 1.47$ to 1.80 ) and 205 (6.9\%) had a cesarean section (PPROM group) versus 2,217 (10\%, controls; RR 0.77; $95 \% \mathrm{Cl} 0.67$ to 0.88 ). Birth weight was higher in the PPROM group (2825 versus 2676 gram, $\mathrm{p}=<0.0001)$. NICU admission was seen more often in the control group than in de PPROM group (3.0\% versus $1.4 \%$, RR 0.45 [95\% Cl 0.32 to 0.63$]$ ). 


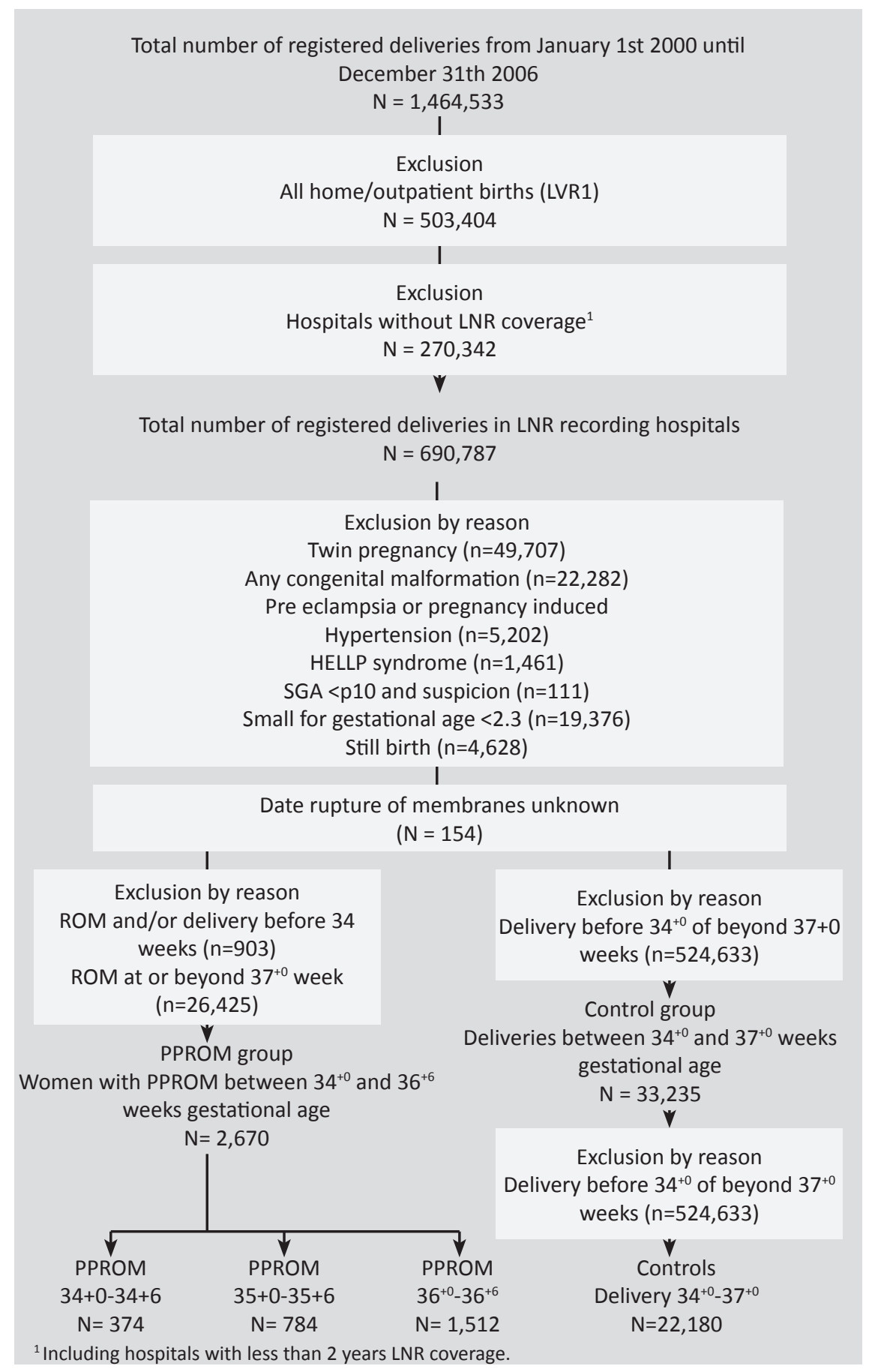

Figure $\mathbf{8 . 1}$ | Selection process 


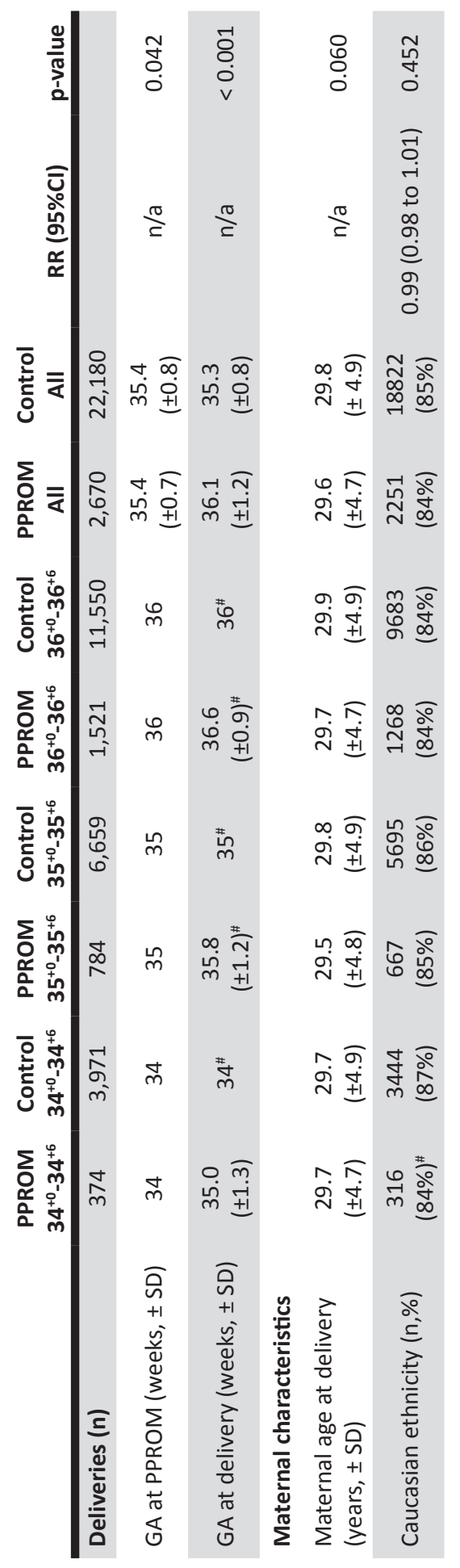



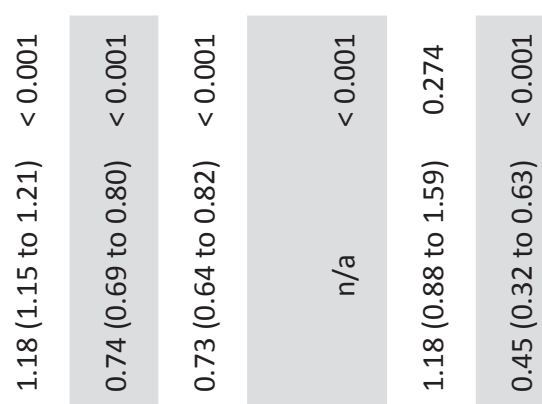

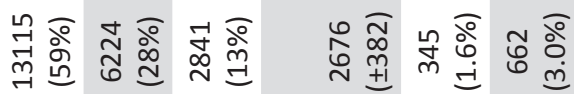
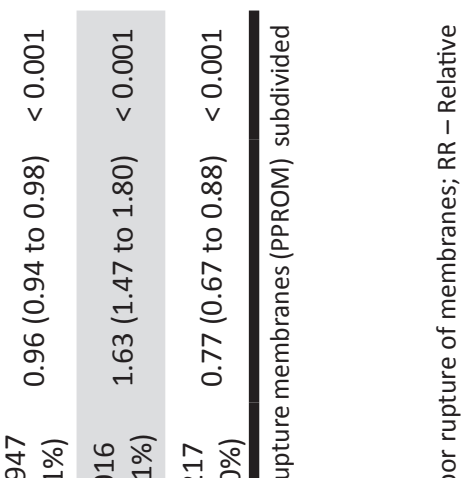

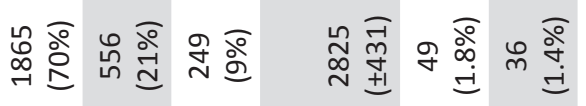

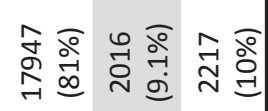

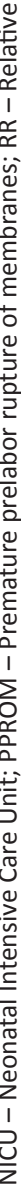

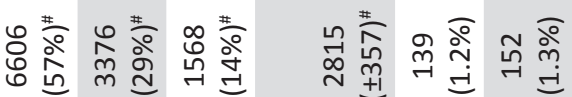

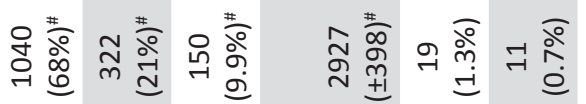

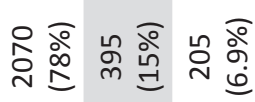

๙

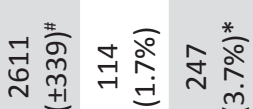

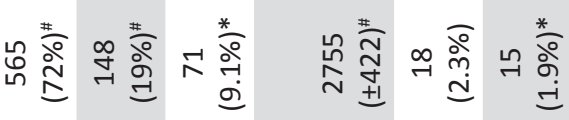

రั

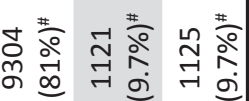

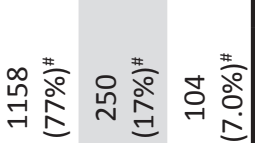

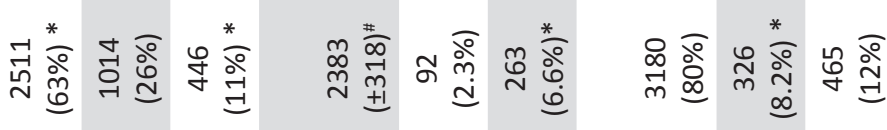

○

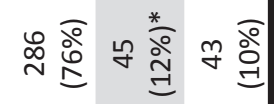

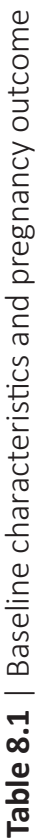




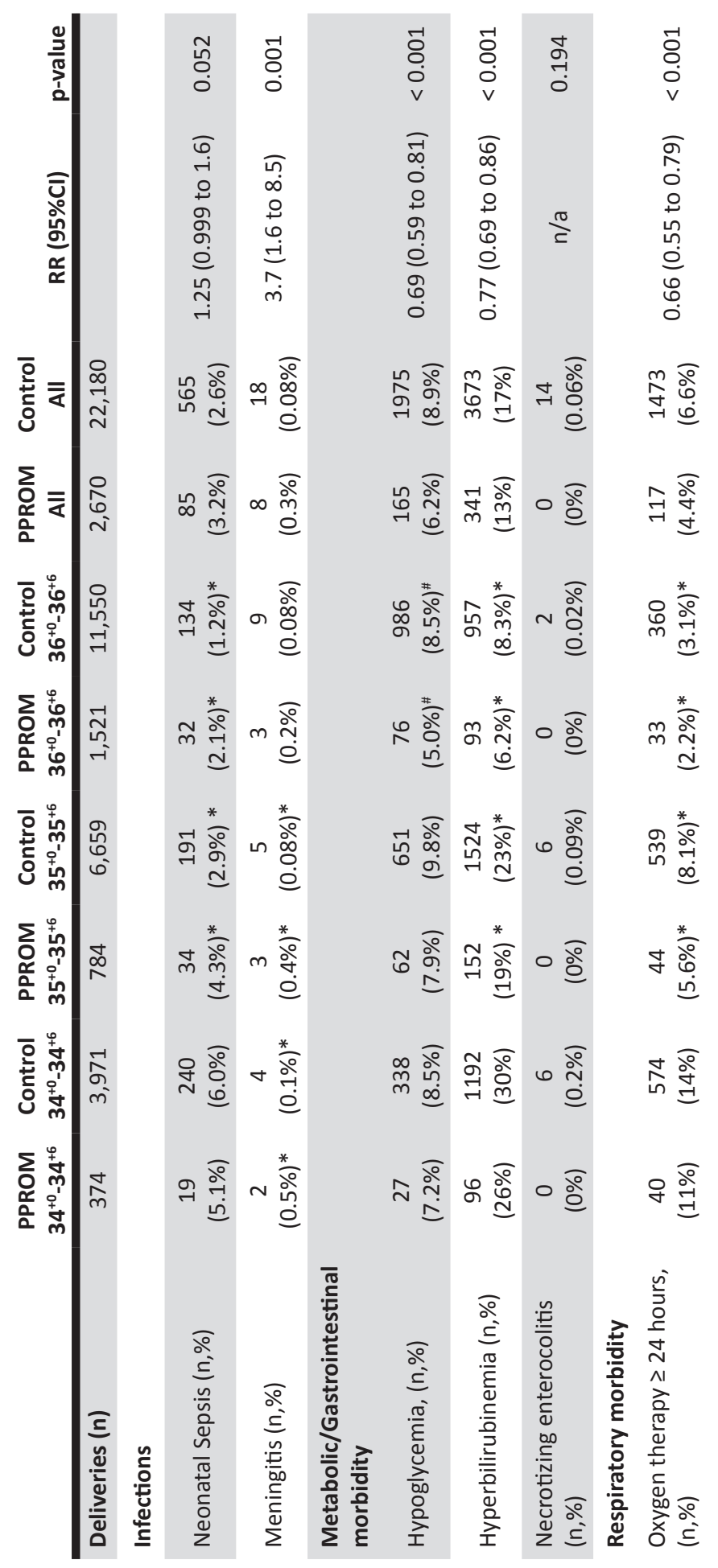




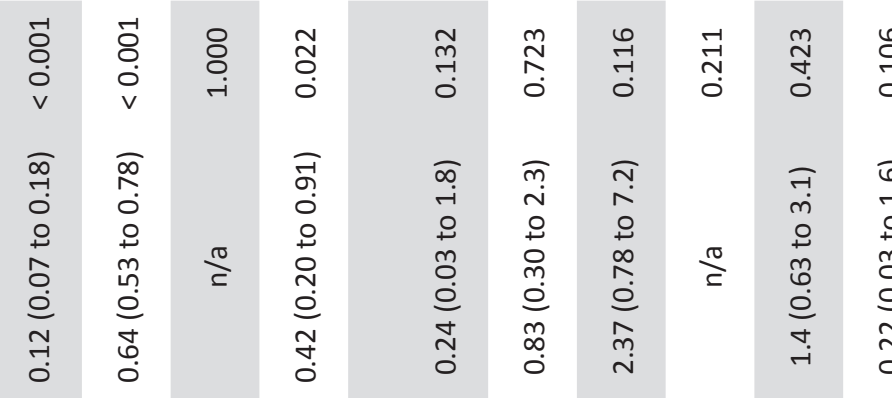

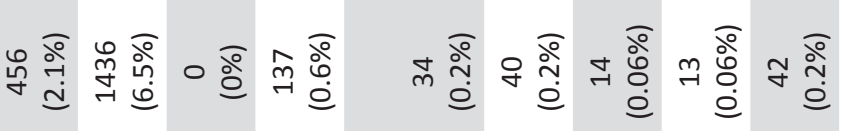

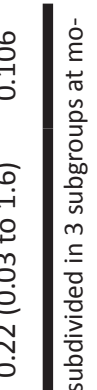

范

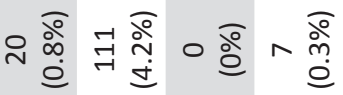

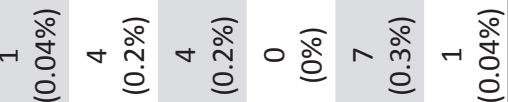

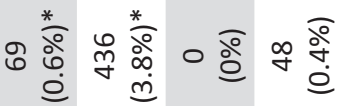

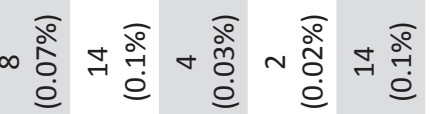

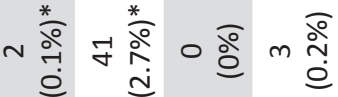

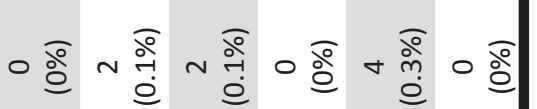

索

$\stackrel{\frac{9}{3}}{\frac{0}{0}}$

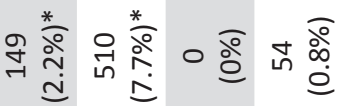

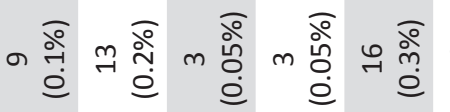

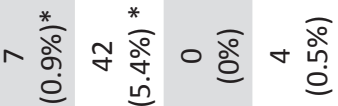

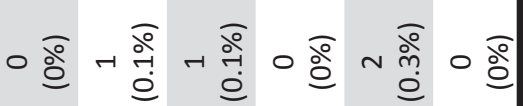

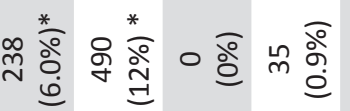

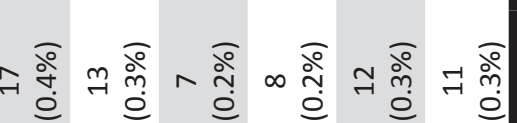

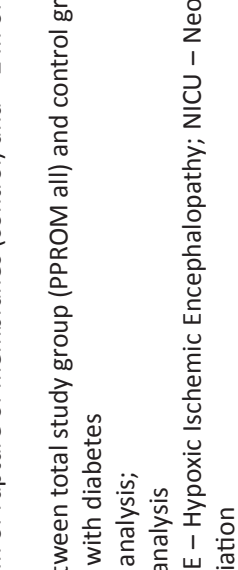

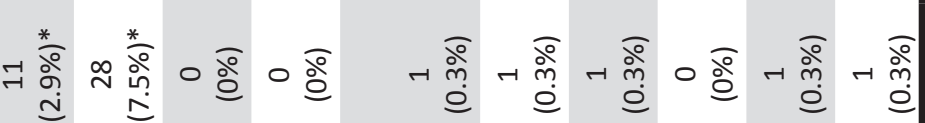

V

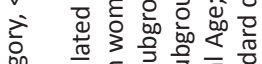

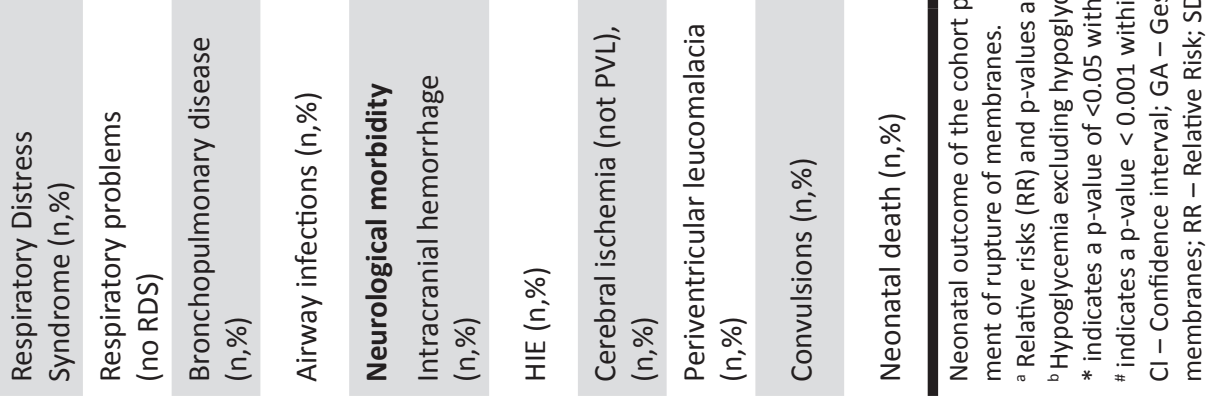




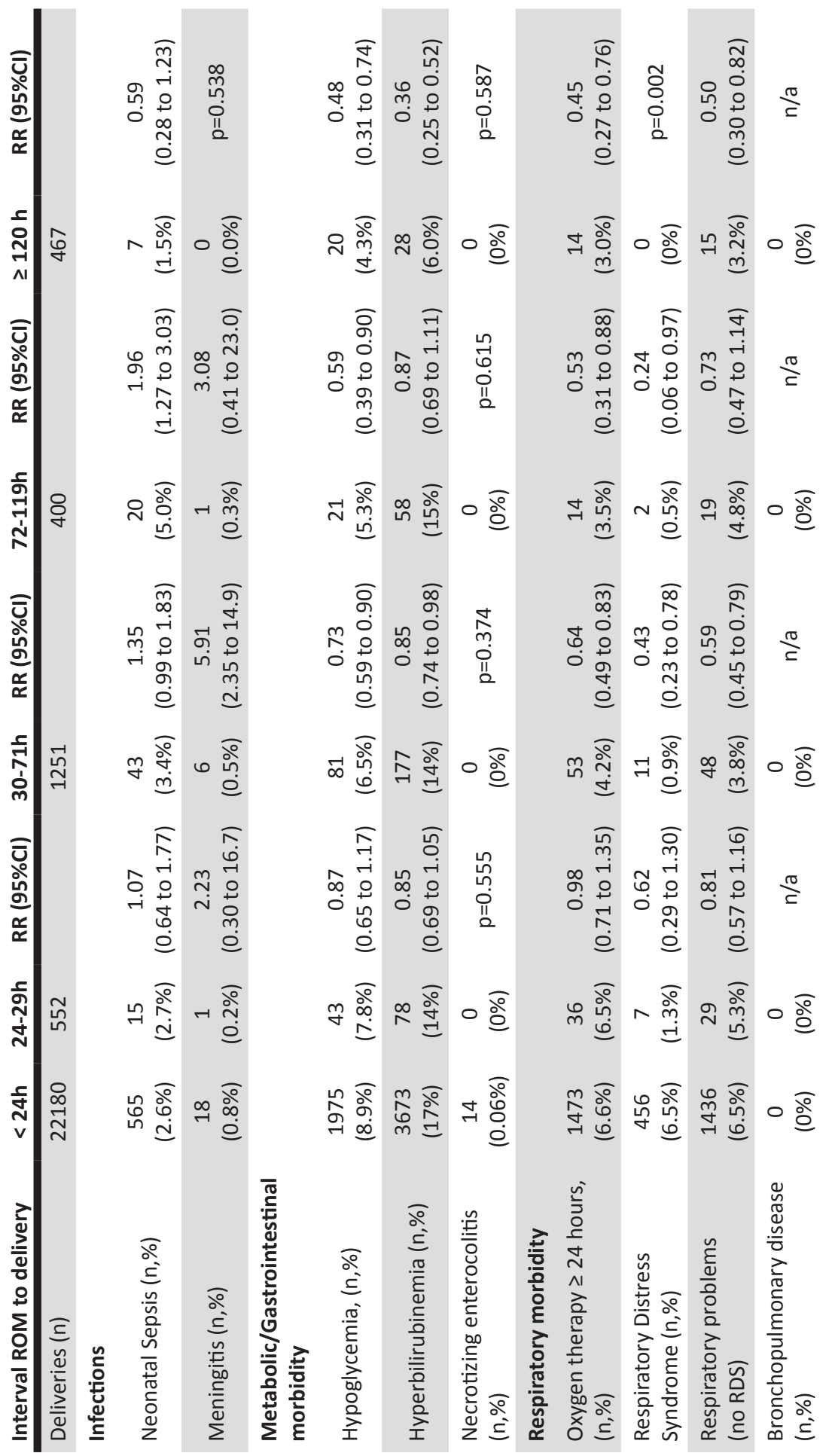




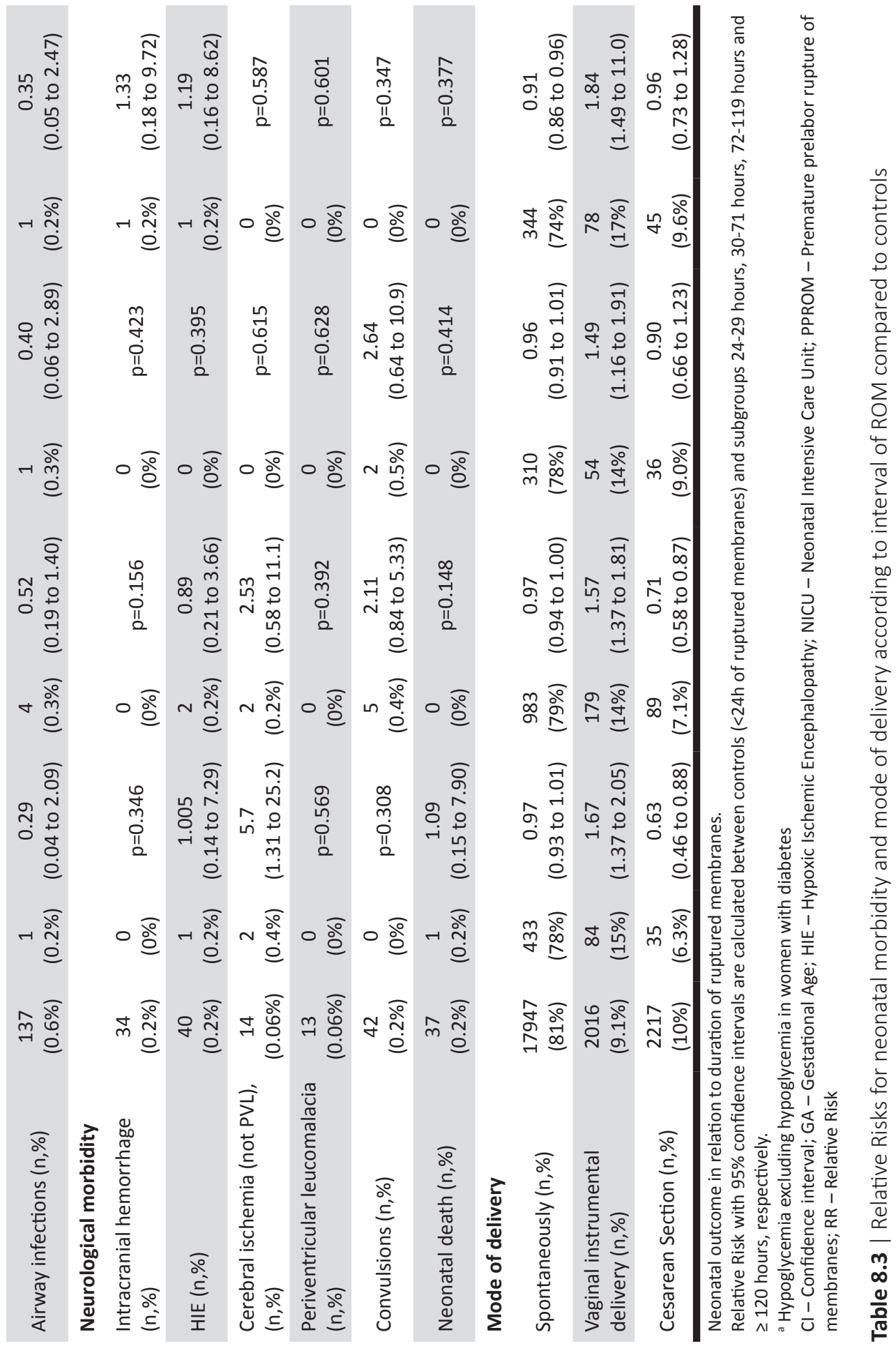




\section{NEONATAL SEPSIS}

Overall 85 (3.2\%) neonates suffered from a neonatal sepsis in the PPROM group versus 565 (2.6\%) in the control group (RR $1.25 ; 95 \% \mathrm{Cl} 0.99$ to 1.56 ). The incidence of sepsis was $5.1 \%$ versus $6.0 \%$ at 34 weeks, $4.3 \%$ versus $2.9 \%$ at 35 weeks, and $2.1 \%$ versus $1.2 \%$ at 36 weeks for women with PPROM and women without PPROM respectively. The adjusted odds of PROM on sepsis was 1.28 (95\% Cl 1.02 to 1.61) (table 2). At 35 weeks of gestation a birth (adjusted OR: 1.61 [95\% Cl 1.08 to 2.35]) and 36 weeks (adjusted OR 1.88 [95\%Cl 1.27 to 2.78$]$ ) the risk of a neonatal sepsis was increased in the PPROM group compared

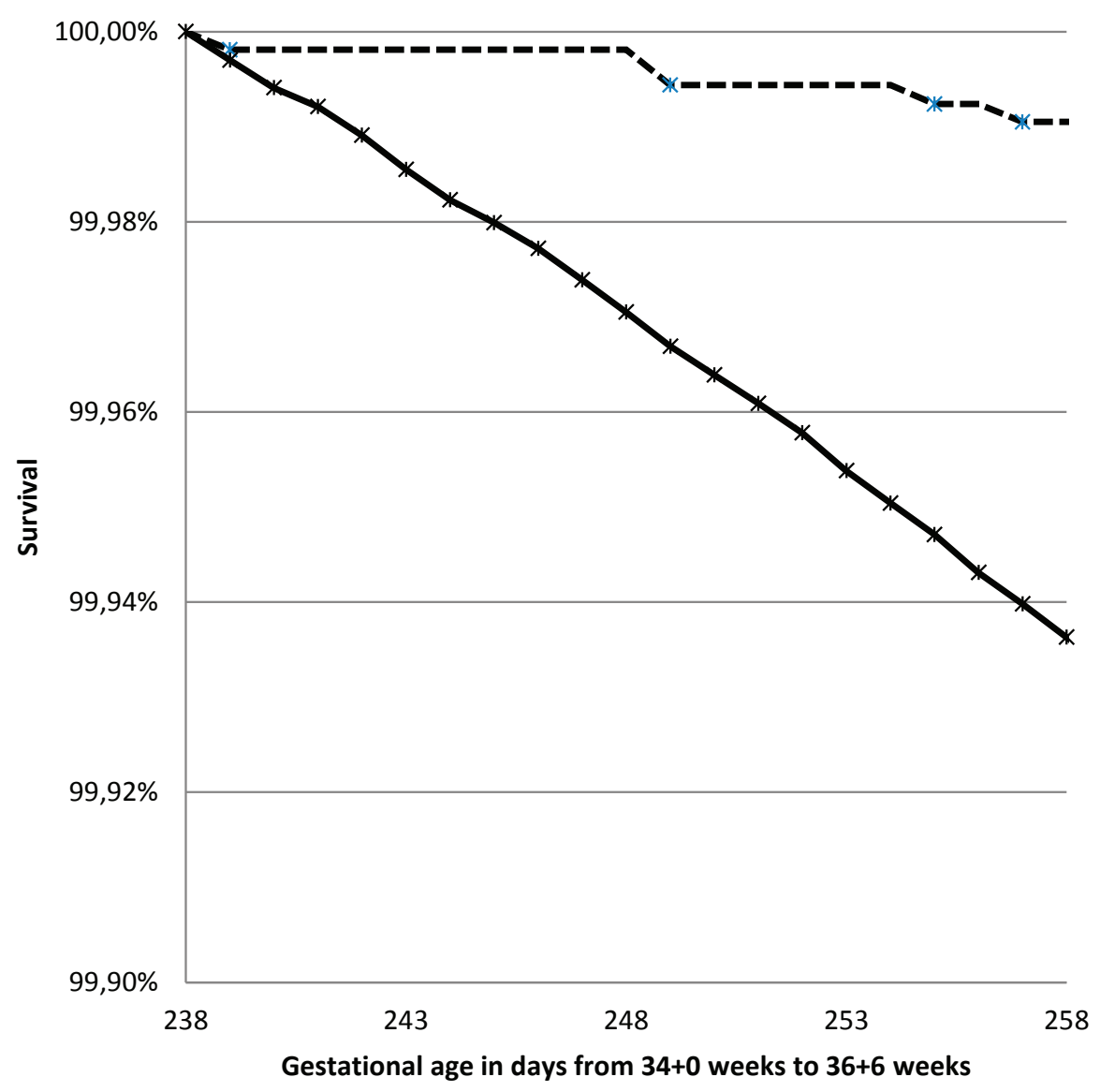

- - PPROM alive $\longrightarrow$ No PPROM alive

Log-Rank Chi-Square 45.5240; Df 1; p-value Chi-Square $<0.0001$

Wilcoxon Chi-Square 39.3837; Df 1; p-value Chi-Square $<0.0001$

-2Log(LR) Chi-Square 72.4446; Df 1; p-value Chi-Square $<0.0001$

Figure 8.2 | Kaplan Meier Curve of overall survival between $34^{+0}$ and $36^{+6}$ weeks gestational age between PROM > 24h before birth and no-PROM 
to controls.

\section{NEONATAL MORBIDITY}

Compared to controls the risk of hypoglycemia (6.2\% versus $8.9 \%)$, hyperbilirubinemia (13\% versus $17 \%), \geq 24$ h oxygen therapy ( $4.4 \%$ versus $6.6 \%)$, respiratory distress syn-

\begin{tabular}{|c|c|c|c|c|}
\hline Term & $\begin{array}{l}\text { PROM } \\
N=53914\end{array}$ & $\begin{array}{l}\text { No PROM } \\
N=1261661\end{array}$ & $\operatorname{RR}(95 \% \mathrm{Cl})$ & $p$-value \\
\hline $\begin{array}{l}\text { 34+0 - 34+6 wks. } \\
\text { n/nTotal, (\%) }\end{array}$ & $\begin{array}{l}1 / 494 \\
(0.2 \%)\end{array}$ & $\begin{array}{c}253 / 8,904 \\
(2,8 \%)\end{array}$ & $\begin{array}{c}0.07 \\
\text { (0.01 to } 0.51)\end{array}$ & 0.0004 \\
\hline $\begin{array}{l}35+0-35+6 \text { wks, } \\
\text { n/nTotal, (\%) }\end{array}$ & $\begin{array}{l}2 / 837 \\
(0.2 \%)\end{array}$ & $\begin{array}{c}275 / 14,490 \\
(1,9 \%)\end{array}$ & $\begin{array}{c}0.13 \\
(0.03 \text { to } 0.51)\end{array}$ & 0.0005 \\
\hline $\begin{array}{l}36+0-36+6 \text { wks, } \\
n / n \text { Total, }(\%)\end{array}$ & $\begin{array}{l}2 / 1890 \\
(0.1 \%)\end{array}$ & $\begin{array}{c}308 / 27,710 \\
(1.1 \%)\end{array}$ & $\begin{array}{c}0.10 \\
(0.02 \text { to } 0.38)\end{array}$ & $<0.0001$ \\
\hline $\begin{array}{l}\text { 37+0 }-37+6 \text { wks, } \\
\text { n/nTotal, (\%) }\end{array}$ & $\begin{array}{c}0 / 4663 \\
(0 \%)\end{array}$ & $\begin{array}{c}392 / 67,810 \\
(0.6 \%)\end{array}$ & $\mathrm{n} / \mathrm{a}$ & $<0.0001$ \\
\hline $\begin{array}{l}38+0-38+6 \text { wks, } \\
n / n \text { Total, }(\%)\end{array}$ & $\begin{array}{c}12 / 8,345 \\
(0.1 \%)\end{array}$ & $\begin{array}{c}464 / 179,606 \\
(0.3 \%)\end{array}$ & $\begin{array}{c}0.56 \\
\text { (0.31 to } 0.99)\end{array}$ & 0.042 \\
\hline $\begin{array}{l}\text { 39+0 - 39+6 wks, } \\
\text { n/nTotal, (\%) }\end{array}$ & $\begin{array}{c}12 / 13,126 \\
(0.09 \%)\end{array}$ & $\begin{array}{c}449 / 296,072 \\
(0.2 \%)\end{array}$ & $\begin{array}{c}0.60 \\
(0.34 \text { to } 1.1)\end{array}$ & 0.080 \\
\hline $\begin{array}{l}\text { 40+0 - 40+6 wks, } \\
\text { n/nTotal, (\%) }\end{array}$ & $\begin{array}{c}9 / 14,746 \\
(0.06 \%)\end{array}$ & $\begin{array}{c}477 / 356,583 \\
(0.1 \%)\end{array}$ & $\begin{array}{c}0.46 \\
(0.24 \text { to } 0.88)\end{array}$ & 0.020 \\
\hline $\begin{array}{l}\text { 41+0-41+6 wks, } \\
\text { n/nTotal, (\%) }\end{array}$ & $\begin{array}{c}12 / 8,652 \\
(0.1 \%)\end{array}$ & $\begin{array}{c}387 / 235,907 \\
(0.2 \%)\end{array}$ & $\begin{array}{c}0.85 \\
\text { (0.48 to } 1.50)\end{array}$ & 0.570 \\
\hline $\begin{array}{l}\text { 42+0-42+6 wks, } \\
\text { n/nTotal, }(\%)\end{array}$ & $\begin{array}{c}5 / 1,038 \\
(0,5 \%)\end{array}$ & $\begin{array}{c}106 / 70,823 \\
(0,2 \%)\end{array}$ & $\begin{array}{c}3.21 \\
(1.2 \text { to } 7.8)\end{array}$ & 0.007 \\
\hline $\begin{array}{l}\text { 42+00 - } 42+6 \text { wks, } \\
\text { n/nTotal, (\%) }\end{array}$ & $\begin{array}{l}0 / 73 \\
(0 \%)\end{array}$ & $\begin{array}{c}2 / 1,479 \\
(0,1 \%)\end{array}$ & $\mathrm{n} / \mathrm{a}$ & 0.750 \\
\hline Overall Total & $\begin{array}{c}55 / 53,8914 \\
(0.1 \%)\end{array}$ & $\begin{array}{c}3,113 / 1,121,661 \\
(0.3 \%)\end{array}$ & $\begin{array}{c}0.41 \\
(0.32 \text { to } 0.54)\end{array}$ & $<0.0001$ \\
\hline Birth weight $>\mathrm{p} 10^{\mathrm{a}}$ & $\begin{array}{c}43 / 51,139 \\
(0.08 \%)\end{array}$ & $\begin{array}{c}2581 / 1,199,237 \\
(0.2 \%)\end{array}$ & $\begin{array}{c}0.40 \\
(0.29 \text { to } 0.57)\end{array}$ & $<0.0001$ \\
\hline Birth weight 2.3-p10 & $\begin{array}{c}12 / 2,775 \\
(0.43 \%)\end{array}$ & $\begin{array}{c}532 / 62,424 \\
(0.85 \%)\end{array}$ & $\begin{array}{c}0.51 \\
(0.29 \text { to } 0.90)\end{array}$ & 0.017 \\
\hline
\end{tabular}

Incidence of stillbirth for all ongoing pregnancies beyond 34 weeks of gestational age per week of gestational age at delivery.

${ }^{a}$ Excluding all small for gestational age infants, defined as a birth weight $<\mathrm{p} 2.3$.

PROM was defined as $>24 \mathrm{~h}$ of ruptured membranes at moment of delivery.

$\mathrm{Cl}$ - Confidence interval; PROM - Prelabor rupture of membranes; RR - relative risk; wks - weeks gestational age at birth

Table 8.4 | Incidence of stillbirth with and without PPROM beyond 34 weeks of gestational age 
drome $(0.8 \%$ versus $2.1 \%)$, respiratory problems (no RDS, $4.2 \%$ versus $6.5 \%$ ), airway infections $(0.3 \%$ versus $0.6 \%)$ was significantly decreased in the PPROM group, whereas the risk for meningitis $(0.3 \%$ versus $0.08 \%)$ was increased. No difference was found for the other neonatal outcomes (Table 8.2).

\section{NEONATAL MORTALITY}

Fourteen $(0.1 \%)$ neonates died in the first week after birth in the control group versus none in the PPROM group ( $p=0.176)$.

\section{LATENCY}

Table 8.3 shows the neonatal outcome and mode of delivery according to the interval between rupture of membranes and birth irrespectively of gestational age at the time of ruptured of membranes. Latency itself had no additional effect on the mode of delivery. Latency did not increase the incidence of neonatal sepsis until 72-119 hours of PPROM. In this period the incidence of neonatal sepsis is significantly increased (RR 1.96 [95\% Cl 1.27 to 3.03]) whereas in women with more than 120 hours PPROM the incidence of neonatal sepsis tends to decrease (RR 0.59 [95\% Cl 0.29 to 1.23]) (Table 8.3) Latency decreased the risk of hypoglycemia (8,9\% (controls), 7.8\% (ROM 24-29h), 6.5\% (30-71h), 5.3\% (72-119h), $4.3 \%(\geq 120 \mathrm{~h})$ ). For RDS a same pattern was seen (2.1\% (controls), $1.3 \%$ (24-29h); $0.9 \%$ (30-71h), 0.5\% (72-119h), 0\% ( $\geq 120 \mathrm{~h})$ ) (Table 8.3).

\section{STILLBIRTH}

As lined out in the methods section we made an additional selection for the risk of stillbirth for women with PROM ( $>24$ hours of ruptured membranes) versus controls ( $<24 \mathrm{~h}$ of ruptured membranes), from 34 weeks gestational onwards. From January $1^{\text {st }} 2000$ until December $31^{\text {st }} 2006,1,464,533$ deliveries were recorded in the LVR-1 and LVR-2 registries. In total 148,958 deliveries had to be excluded due to multiple pregnancies ( $n=57,773$ ), congenital anomalies $(n=33,525)$, pre-eclampsia or HELLP syndrome $(n=8,968)$, severe growth restriction $<p 2.3(n=29,014)$, date of ROM undetermined $(n=112)$, delivered before $34^{+0}$ weeks $G A(n=19,122)$ and 43 weeks or more $(n=320)$. Leaving 1,315,575 women available for analysis. Of these women 53,914 (4.1\%) had ROM for $>24$ hours and $1,261.661$ had $\mathrm{ROM}<24$ hours.

From 34 weeks onwards there were 3,168 stillbirths, this was significantly decreased $(55 / 53,914 ; 0,10 \%)$ in the ROM group compared to the $(3,113 / 1,261,661 ; 0,25 \%)$ in the non ROM group (RR 0.41 [95\% Cl: 0.32 to 0.54 ]). Figure 8.2 represents the Kaplan-Meier survival curve between women with PPROM and women without PPROM between $34^{+0}$ weeks GA (238 days) and $36^{+6}$ weeks GA (258 days). Between $34^{+0}$ and $36^{+6}$ weeks GA 
$3,211(6,0 \%)$ women in the PROM group delivered and 5 stillbirth occurred (cumulative stillbirth rate at $36^{+6}$ weeks $0,009 \%$ ) during this period. In the control group 51,104 (4.1\%) delivered and 836 stillbirth occurred (cumulative stillbirth rate at $36^{+6}$ weeks 0,066\%). Table 8.4 shows the risk of stillbirth per week of gestational age at moment of delivery. Overall the adjusted odds ratio of ROM for $>24$ hours for antepartum death or stillbirth was OR 0.38 (95\% Cl 0.29-0.50), compared to ROM for <24 hours.

\section{SUBGROUP ANALYSIS}

Subgroup analysis for all outcome were performed according to gestational age and latency, subdivided in 34, 35 and 36 weeks (Appendix Table A.1-A.3), as well as a subgroup analysis for latency in which a short interval of PPROM (24 until 29 hours) was compared to longer interval of PPROM. (Appendix Table A.4)

Overall we did not find abnormal patterns. Lack of significance in the subgroups was mainly due to the small numbers. Neonatal morbidity decreased in both groups (PPROM and controls) at increasing gestational age.

With respect to latency, the incidence of neonatal sepsis was increased at 35 and 36 weeks of gestational age compared to controls ( $<24$ hours of PPROM). This difference seems to be related to the gestational age period rather than to the interval of ruptured membranes (See Appendix Table A.5-A.7).

Compared to the shortest interval of PPROM subgroup (24-29 hours), latency did not increase the incidence of neonatal sepsis within the PPROM group.

\section{DISCUSSION}

In this large retrospective cohort we analyzed the neonatal morbidity on preterm women with premature rupture of membranes between $34^{+0}$ and $36^{+6}$ weeks GA compared to spontaneous deliveries in the same period. The overall incidence of neonatal sepsis in women with PPROM was not different compared to controls and prolonged rupture of membranes was not related to an increased stillbirth rate.

The strength of this current study is its size. Over a recent period (1999-2006) we were able to identify 2,670 cases with late preterm PPROM. Data are derived form a reliable and validated population-based data system. It includes almost all deliveries in The Netherlands and is therefore a good reflection of the clinical practice. We decided not to include the year 2007 because of the fact that at that moment the PPROMEXIL trial ${ }^{89}$ was running in 60 out of 98 (61\%) hospitals across The Netherlands. Randomization between induction of labor versus expectant management might have influenced the rather conservative treatment strategy until then. Until 2006, the preferred management in The 
Netherlands in case of PPROM was expectant management.

To our knowledge this is the largest cohort of patients with late preterm PPROM and out weight by far recent large $\mathrm{RCT}^{\prime} \mathrm{s}^{89,148}$ giving us the possibility to analyze neonatal outcome with very low incidences as well as neonatal mortality.

The study has also its limitations. The accuracy of the data depends on the preciseness of the registration of obstetricians and pediatricians. The size of the national database minimizes this influence. Since the LNR-registry is not mandatory in general hospitals (LNR-registry is mandatory only in tertiary hospitals) we had to exclude $37 \%$ of the cohort

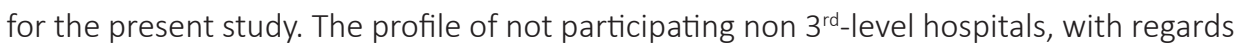
to size, distribution and financial support, does not differ from participating general hospitals and we therefore assume that these missing data has no influence on the incidence of neonatal outcome.

The LVR registry did not record PPROM as a sole item. We therefore had to calculate the interval of ruptured membranes. We arbitrarily decided to assign women with more than 24 hours interval between rupture of membranes and delivery as the PPROM group and those with less than 24 hours as controls. Therefore women with PPROM for several hours might have been assigned to the control group, whereas a prolonged delivery without rupture of membranes before onset of labor might have been assigned to the PPROM group. However we expect that this might have been only a small number of women and therefore did not influence the outcome of this study.

We decided to use spontaneous deliveries between 34 and 37 weeks as controls. Although we excluded patients with hypertensive disorders, intrauterine growth restriction, congenital malformations and induction of labor or a planned cesarean section it can be debated whether this is a normal control group. There may be another unknown reason why these women delivered prematurely which could have contributed on the outcome of these neonates. Our findings are in line with two recent RCT's from The Netherlands $s^{89,148}$ and confirm the findings of a recent Cochrane review. ${ }^{19}$

The incidence of neonatal sepsis was comparable to the incidence found in the PPROMEXIL trials. ${ }^{89,148}$ Interestingly the incidence of neonatal sepsis was significantly higher in the 35 and 36 week subgroup compared to controls. However, within the PPROM group itself, latency did not seem to increase the incidence in these subgroups. It may be possible that PPROM itself is a result of an increased risk for an infection rather than prolonged ruptured membranes.

There is an increased risk for neonatal sepsis when membranes are ruptured between 72 and 119 hours before delivery, however, a sharp decline in incidence is noticed after 120 hours. We feel that, although significant, the increased risk of neonatal sepsis between 72 and 119 hours should be interpreted with some caution. Especially because of the 
fact that, apart from meningitis, all other neonatal outcome showed either an decreased incidence or did not differ when the interval of PPROM prolonged and we did not find any neonatal death in the PPROM group versus 14 in the control group. These findings are in line with the PPROMEXIL trials. ${ }^{89,148}$

Our study demonstrates that prolonged rupture of membranes does not increase the risk of stillbirth, as we even found a lower stillbirth rate in this category. The latter phenomenon might be explained by the fact that, although treated expectantly, women were monitored more precisely from the moment of ruptured membranes than women with an on-going pregnancy without ruptured membranes.

In contrast with prospective trials, we were not able to report the incidence of chorioamnionitis because this item is not registered in the PRN database. It is, however, known that the incidence of chorioamnionitis is significantly higher in women with a longer interval between ROM and delivery. ${ }^{20-24,89,148}$ Although a relationship between chorioamnionitis and adverse neonatal outcome has been shown, this was only done in a very premature population, and we doubt if these findings can be applied to the near-term PPROM population. ${ }^{135}$

We are not able to combine the PRN data with long term follow-up data of these children. However, it has been reported that there is a strong, dose-dependent relationship between gestational age at delivery and need for special education. ${ }^{143}$

Taking all the evidence into account, this large retrospective cohort study in women with late preterm PPROM, supports the growing evidence of recent trials and meta-analysis on this issue that prolongation of pregnancy by managing a women expectantly will not compromise pregnancy outcome nor will it lead to an increased risk of neonatal sepsis. Expectant management reduces the risks of prematurity other than neonatal sepsis, without an increased risk for stillbirth or neonatal death. We therefore conclude that women with late preterm rupture of membranes, without any signs of infection or fetal distress, can treated expectantly under strict monitoring. 


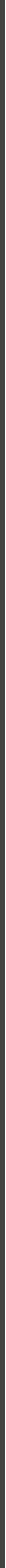




\section{CHAPTER}

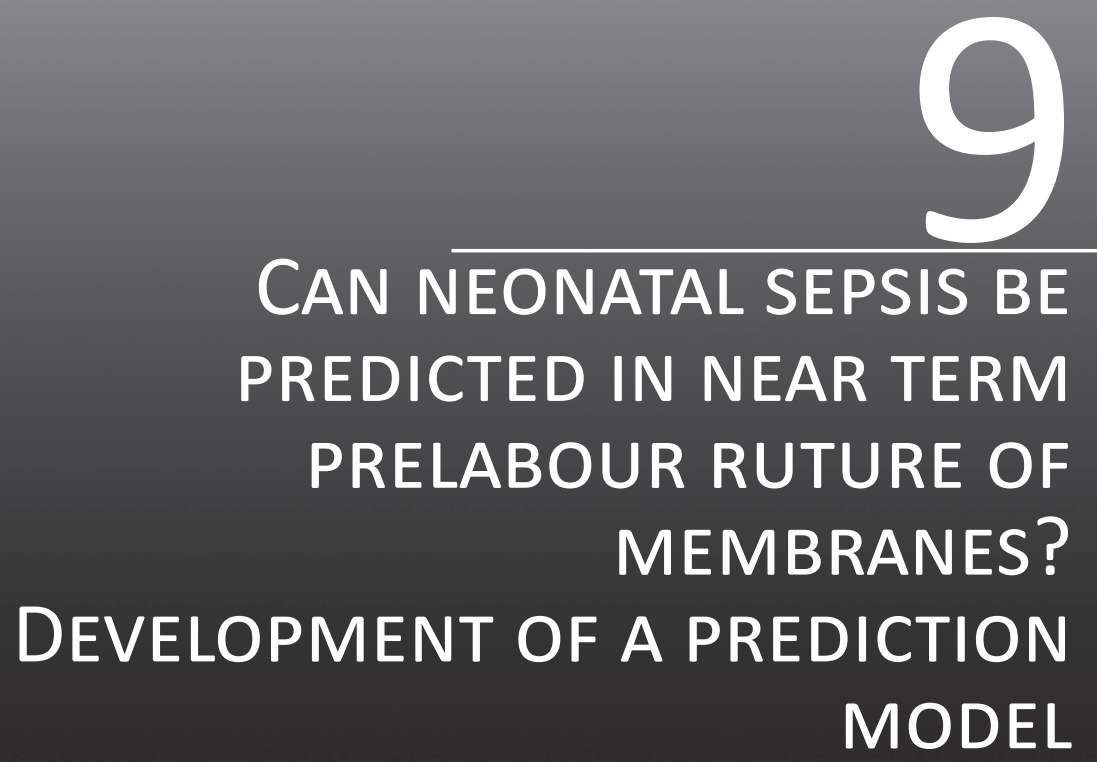

David P van der Ham, Sander van Kuijk, Brent C Opmeer, Christine Willekes, J (Hans) J van Beek, Antonius LM Mulder, Aren van Loon, Martiët Groenewout,

Gerald Mantel, Kitty WM Bloemenkamp, Martina Porath, Anneke Kwee, Bettina Akerboom, Dimitri NM Papatsonis, Godfried CH Metz, Jan G Nijhuis,

Ben WJ Mol

Submitted 


\section{ABSTRACT}

\section{OBJECTIVE}

Women with preterm prelabour rupture of membranes (PPROM) have an increased risk that their child will develop neonatal sepsis. We evaluated whether neonatal sepsis can be predicted from ante partum parameters in women with PPROM.

\section{DESIGN}

We used multivariable logistic regression to develop a prediction model.

\section{POPULATION}

Data from two PPROMEXIL trials, in which women with PPROM (> 24 hours) between 34 and 37 weeks of gestational age who had been randomised for immediate delivery or expectant management was used.

\section{METHODS}

We evaluated 13 potential antepartum predictors for neonatal sepsis. Missing data were imputed. Discriminative ability of the model was expressed as the area under the receiver operating characteristic (ROC) curve and a calibration with both a calibration plot and the Hosmer and Lemeshow goodness-of-fit test. Overall performance of the prediction model was quantified as the scaled Brier score. The model was internally validated by bootstrapping.

\section{RESULTS}

We studied 970 women of which 33 (3.4\%) neonates suffered neonatal sepsis. Maternal age (OR 1.09 per year), maternal C-reactive protein level (OR 1.01 per $\mathrm{mmol} / \mathrm{l}$ ) maternal temperature (OR 1.80 per ${ }^{\circ} \mathrm{Celcius}$ ) and positive Group B streptococcus culture (OR 2.20) were associated with an increased risk of neonatal sepsis. The model had an area under the ROC-curve of 0.71 (95\% confidence interval 0.61 to 0.82 ). The goodness-to-fit ( $p=0.32$ ) and adjusted Brier score $30 \%$ verified both good calibration and accuracy of the model. 


\section{CONCLUSIONS}

In women with PPROM near term, the risk of neonatal sepsis can more precisely be estimated from antepartum parameters. 


\section{INTRODUCTION}

Preterm prelabour rupture of the membranes (PPROM) is an important clinical problem. It complicates 1 to $5 \%$ of all pregnancies, and precedes $30-40 \%$ of all preterm deliveries. ${ }^{30-32}$ PPROM is associated with increased fetal and maternal morbidity and mortality. ${ }^{1,2,9}$ The risk of infection, resulting in chorioamnionitis and neonatal sepsis, is considered to be the largest threat in patients with PPROM near term. When there is no need to administer antenatal corticosteroids for lung maturity, international guidelines recommend induction of labour from 34 weeks onwards..$^{15,16}$

However, two consecutive large multicentre, open-label randomised controlled trials in The Netherlands showed that induction of labour ( $\mathrm{OL}$ ) did not reduce neonatal sepsis compared to expectant management (EM) (combined Relative Risk (RR) 0.66, 95\% Confidence Interval (CI) 0.30 to 1.5$),{ }^{89,148}$ with an average neonatal sepsis of $2.7 \%$ in the loLgroup and $3.8 \%$ in the EM-group.

Nevertheless, because of the potentially life threatening risk of neonatal sepsis, identification of neonates at increased risk for this complication is crucial. Identification of a high risk subgroup in women with PPROM between 34 and 37 weeks could be helpful in the management of these women. Inducing labour and close monitoring of neonates at risk may reduce the incidence of neonatal sepsis, whereas expectant management and less frequent monitoring in low risk patients will potentially lead to a reduction of neonatal complications due to prematurity and a reduction in costs.

In the present study, we assessed the capacity of ante partum clinical characteristics to predict neonatal sepsis in order to develop a prediction model for the prediction of neonatal sepsis, based on predictors that are easily available in routine practice.

\section{METHODS}

\section{PATIENT POPULATION}

For this present study we used the data from the PPROMEXIL trials; PPROMEXIL (ISRCTN29313500) ${ }^{89}$ and PPROMEXIL-2-trial (ISRCTN05689407). ${ }^{148}$ The PPROMEXIL trials were multicentre, open-label, randomised controlled trials in The Netherlands in which all eight academic and 52 nonacademic hospitals participated. In short, women with a singleton or twin pregnancy were eligible for the PPROMEXIL trial when they were not in labour 24 hours after PPROM between 34 and 37 weeks of gestational age. Figure 1 outlines the study design and selection of patients.

PPROM had to be occurred after $26^{+0}$ weeks. Women with a monochorionic multiple pregnancy, an abnormal (non-reassuring) cardiotocogram, meconium stained amniotic 


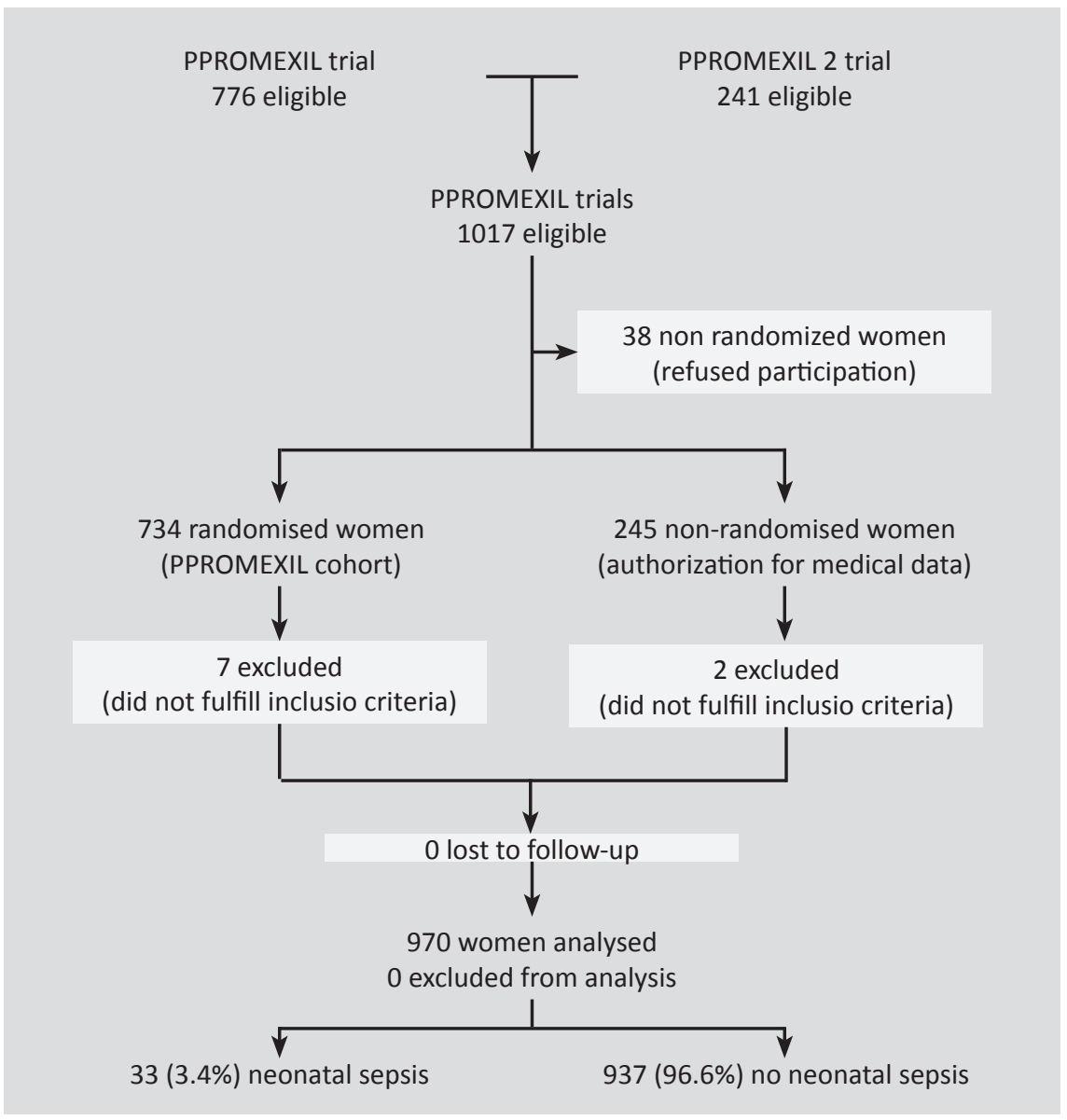

Figure 9.1 | Patient selection process

fluid, major fetal anomalies, signs of intrauterine infections HELLP syndrome or severe pre-eclampsia were not included.

Women who had consented with participation in the trial were randomly allocated to either induction of labour (loL-group) or expectant management (EM-group). Patients who had not given informed consent but who provided authorisation for using their medical data, were treated according to local protocol (non-randomisation group), and had either immediate delivery or expectant monitoring. In the present study we combined data from randomised women and women who refused randomisation in one cohort.

Immediate delivery was mostly due to induction of labour, but could also occur after planned Caesarean section, for example for breech delivery. Expectant management consisted of at least daily maternal temperature monitoring and twice weekly blood sampling 
for signs of infection. If a woman reached $37^{+0}$ weeks of gestational age labour was induced. For a more detailed description of the interventions we refer to the trial protocol that has been published before. ${ }^{27}$

The outcome of interest was neonatal sepsis. This was defined as: (1) positive blood culture taken at birth (not staphylococcus epidermidis) or (2) 2 or more symptoms of infection within 72 hours (apnea, temperature instability, lethargy, feeding intolerance, respiratory distress, haemodynamic instability) plus one of three items: (a) positive blood culture (culture proven sepsis); (b) CRP > 20 (suspicion sepsis); (c) positive surface culture of a known virulent pathogen (suspicion sepsis). ${ }^{89,148}$ Each case of suspected sepsis was judged by an independent panel of paediatricians (A. L. M. M. and R. M.) who, unaware of the allocation of randomisation, adjudicated between neonatal sepsis (proven or suspected sepsis) or no sepsis.

\section{CANDIDATE PREDICTORS}

There are guidelines about the number of potential predictors that can be validly included in the prediction model. Often, a rule of 10 events per predictor variable is proposed. ${ }^{157}$ However, since this is the first study to assess the predictive value of a multivariable model on the probability of neonatal sepsis, with many more potential predictors, we allowed five events per predictor variable to be included in the final model, dictating a maximum of six predictors $\left(\mathrm{N}_{\text {cases }}=33\right.$ ). We assumed the following variables as candidate predictors: maternal age, parity (nulli parity versus multiple parity), ethnicity, maternal smoking, maternal body mass index (BMI) at start of pregnancy, gestational age (GA) at PPROM, antenatal administration of steroids, on admission maternal C-reactive protein (CRP) level, maternal white blood cell count, maternal temperature, positive vaginal culture (any pathogenic specimen, i.e. coagulase-negative Staphylococcus or Escheria coli ), positive group B streptococcus (GBS) culture and ante partum administration of antibiotics. Antibiotics were given according to local protocol at admission, before signs or symptoms of chorioamnionitis.

\section{MOdEL DEVELOPMENT}

Data were incomplete for some predictor variables. The omission of patients from the analysis who have one or more predictor values missing could lead to a loss of precision and subsequently a reduction in statistical power. ${ }^{158-160}$ More seriously, using only complete cases could potentially bias results. We assumed these data were missing at random (MAR), meaning that the probability of values being missed is related to the observed values of other variables in the model, including the outcome variable. ${ }^{161}$ Therefore, we imputed the dataset using regression imputation. 
All potential predictors were introduced in a multivariable logistic regression model, and were step-by-step excluded using the Wald test. We used a liberal p-value of 0.20 to keep a predictor in the model, according to current guidelines for developing prediction models. ${ }^{157}$ Ultimately, only a maximum of 6 predictors could be included in the model, so if there were more than 6 significant predictors, the six predictors with the lowest $p$-value would be retained in the model.

There were 14 twin pregnancies in the entire study group, of which 3 of the 28 neonates developed a sepsis, in one case a first born child and in one case both children. Adding both twins to the analysis would introduce high correlation between these pairs, introducing much more complexity to the prediction model. Therefore, in case of a twin pregnancy, we only included the firstborn in the analysis.

\section{INTERNAL VALIDATION}

In deriving our prediction model, we have chosen to include more potential predictors than advised, increasing the risk of severe over-fitting of our model (i.e. overestimation of the coefficients resulting in too extreme predictions for future patients). To account for this, the model was internally validated using bootstrapping techniques, and applied shrinkage to the original prediction model, a method which has been extensively been described by Steyerberg et al. ${ }^{160}$

\section{MODEL PERFORMANCE}

Discriminative ability of the model (after shrinkage) was determined by calculating the area under the receiver operating characteristic (ROC) curve, which is a measure of the models' ability to distinguish between cases with and without neonatal sepsis. This measure can range from 0.5 (no discrimination) to 1.0 (perfect discrimination). ${ }^{162}$ We assessed the calibration of the model with a calibration plot. In a perfect calibration plot the observed probabilities equal the predicted probabilities. In such a scenario, all points would be situated on the line that describes $X=Y$, a 45 -degree line originating from $(0,0)$. To quantify the calibration, we performed a Hosmer and Lemeshow test for goodness-of-fit, where a low $p$-value $(P<0.05)$ indicates evidence of poor calibration and lack of fit. Furthermore, we visually inspected the calibration plot. For overall performance, we computed the Brier score, which is a measure of the accuracy of the predictions. Because the maximum Brier score for a given set of patients depends on the incidence of the outcome, we scaled the Brier score by its maximum possible score for the incidence of neonatal sepsis in our population, so that it ranges from 0 to $100 \%$, and can be interpreted as Pearson's R2 statistic. ${ }^{163}$

We used SPSS for Windows (version 19.0; SPSS Inc., Chicago, Illinois) for the imputation 


\begin{tabular}{|c|c|c|c|}
\hline Characteristics $^{\mathrm{a}}$ & $\begin{array}{c}\text { Women with a } \\
\text { newborn with } \\
\text { neonatal sepsis } \\
(n=33)\end{array}$ & $\begin{array}{l}\text { Women with a } \\
\text { newborn without } \\
\text { neonatal sepsis } \\
(n=937)\end{array}$ & p-value \\
\hline Maternal age (range) $[ \pm S D], y$ & $\begin{array}{c}33.2 \\
(22.9-46.7)[ \pm 6.0]\end{array}$ & $\begin{array}{c}30.2 \\
(18.1-45.5)[ \pm 5.2]\end{array}$ & 0.001 \\
\hline Number of nulliparous, $n$ (\%) & $17(52 \%)$ & 415 (44\%) & 0.415 \\
\hline Twin pregnancy, n (\%) & $2(6.1 \%)$ & $12(1 / 3 \%)$ & 0.024 \\
\hline \multicolumn{4}{|l|}{ Aimed manegement at PPROM } \\
\hline Immediate delivery, $\mathrm{n}(\%)$ & $11(2.9 \%)$ & $369(97 \%)$ & \\
\hline Expectant management, $\mathrm{n}(\%)$ & $22(3.7 \%)$ & $568(96 \%)$ & \\
\hline Ethnic origin, white, $n$ (\%) & 25 (83\%) & 715 (83\%) & 0.913 \\
\hline Maternal smoking, $n$ (\%) & $4(12 \%)$ & $187(20 \%)$ & 0.275 \\
\hline $\begin{array}{l}\text { Higher educational level (higher pro- } \\
\text { fessional school or university), } n(\%)\end{array}$ & $8(38 \%)$ & $195(37 \%)$ & 0.883 \\
\hline $\begin{array}{l}\text { Body mass index (range) [ } \pm \mathrm{SD}] . \mathrm{kg} / \\
\mathrm{m}^{2}\end{array}$ & $\begin{array}{c}25.1 \\
(17.9-43.4)[ \pm 6.0]\end{array}$ & $\begin{array}{c}24.8 \\
(15.8-53.3)[ \pm 5.4]\end{array}$ & 0.799 \\
\hline \multicolumn{4}{|l|}{ Gestational age at PPROM } \\
\hline < 34 weels, $\mathrm{n}(\%)$ & $7(21 \%)$ & $168(18 \%)$ & \multirow{4}{*}{0.895} \\
\hline $34+0$ to $34+6$ weeks, $n$ (\%) & $3(9.1 \%)$ & $141(15 \%)$ & \\
\hline $35+0$ to $35+6$ weeks, $n$ (\%) & $10(30 \%)$ & $268(31 \%)$ & \\
\hline $36+0$ to $36+6$ weeks, $n$ (\%) & $13(39 \%)$ & $338(36 \%)$ & \\
\hline $\begin{array}{l}\text { Gestational age PPROM, mean [ } \pm S D] \\
\text { (median) [IQR], d }\end{array}$ & $\begin{array}{c}244.0[ \pm 14](247) \\
{[241-253]}\end{array}$ & $\begin{array}{c}244.8[ \pm 11](248) \\
{[241-253]}\end{array}$ & 0.693 \\
\hline $\begin{array}{l}\text { Gestational age at delivery, mean } \\
{[ \pm \mathrm{SD}] \text { (median) [IQR], } d}\end{array}$ & $\begin{array}{c}251.1[ \pm 7.1](254) \\
{[246-256]}\end{array}$ & $\begin{array}{c}252.5[ \pm 7.9](254) \\
{[249-258]}\end{array}$ & 0.317 \\
\hline Maternal temperature $\left({ }^{\circ} \mathrm{C}\right),[ \pm S D]$ & $37.5[ \pm 1.0]$ & $37.0[ \pm 0.6]$ & $<0.0001$ \\
\hline Maternal CRP $(\mathrm{mmol} / \mathrm{I}),[ \pm S D]$ & $36.2[ \pm 62]$ & $15.7[ \pm 28]$ & 0.001 \\
\hline Maternal WBC $\left(x 10^{9} / I\right),[ \pm S D]$ & $16.0[ \pm 6.6]$ & $13.2[ \pm 5.2]$ & 0.010 \\
\hline $\begin{array}{l}\text { Positive vaginal culture (any speci- } \\
\text { men), } n(\%)\end{array}$ & $13(40 \%)$ & 205 (22\%) & 0.018 \\
\hline Positive vaginal culture (GBS), $n$ (\%) & $11(33 \%)$ & $143(15 \%)$ & 0.005 \\
\hline $\begin{array}{l}\text { Antenatal corticosteroids adminis- } \\
\text { tration, } n(\%)\end{array}$ & $8(28 \%)$ & $165(18 \%)$ & 0.229 \\
\hline $\begin{array}{l}\text { Antepartum antibiotic treatment, } \\
n(\%)\end{array}$ & $16(48 \%)$ & 364 (39\%) & 0.265 \\
\hline
\end{tabular}

Characteristics indicated in italics are potential predictor characteristics

a Percents given are related to available data per characteristic before imputation and may differ from total number of patiens. CRP - C-reactive protein; GBS - group B streptococcus; IQR - inter quartile range; SD - standard deviation; WBC - white blood count

Table 9.1 | Baseline characteristics and potential predictors 
of missing values. All statistical modelling steps, including internal validation were performed using R, a language and environment for statistical computing (version 2.12.2; http://www.r-project.org/).

\section{RESULTS}

\section{PATIENT POPULATION}

A total of 970 women were identified as eligible for the present study, of whom 727 had been randomised and 243 had not been randomised, but had been managed according to the policy of their preference. From these 970 women, 590 were initially managed expectantly while in 380 the aim had been immediate delivery. Baseline characteristics and distribution of potential predictors between women with and women without a neonate suffering from neonatal sepsis before imputation are given in table 9.1. Of all neonates 33 (3.4\%) suffered a neonatal sepsis.

\section{MODEL DEVELOPMENT AND PERFORMANCE}

Table 9.2 shows both the original prediction model for neonatal sepsis as well as the validated model after the shrinkage. The bootstrap validation yielded a shrinkage factor of 0.89 , and this was uniformly applied to all regression coefficients. Maternal age, C-

\begin{tabular}{lccccc} 
& & original model & & \multicolumn{2}{c}{$\begin{array}{c}\text { model after } \\
\text { internal validation }\end{array}$} \\
Variable & $\begin{array}{c}\text { regression } \\
\text { coefficient }\end{array}$ & $\begin{array}{c}\text { odds ratio } \\
(95 \% \mathrm{Cl})\end{array}$ & p-value & $\begin{array}{c}\text { regression } \\
\text { coefficient }^{\mathrm{a}}\end{array}$ & odds ratio \\
\hline Intercept & -31.17 & - & & -28.06 & \\
\hline $\mathrm{CRP}(\mathrm{mmol} / \mathrm{l})$ & 0.007 & $1.01(1.00-1.01)$ & 0.07 & 0.006 & 1.01 \\
\hline Age (years) & 0.094 & $1.10(1.03-1.17)$ & 0.01 & 0.083 & 1.09 \\
\hline $\begin{array}{l}\text { GBS culture } \\
\text { (pos/neg) }\end{array}$ & 0.886 & $2.42(1.12-5.25)$ & 0.02 & 0.788 & 2.20 \\
\hline Temperature $\left({ }^{\circ} \mathrm{C}\right)$ & 0.658 & $1.93(1.17-3.19)$ & 0.01 & 0.586 & 1.80 \\
\hline
\end{tabular}

To calculate the absolute risk for neonatal sepsis:

$$
\begin{gathered}
P_{\text {sepsis }}=1 /(1+\exp (-(-28.06+0.006 * \mathrm{CRP}+0.083 * \text { Age }+0.788 * \\
\text { GBS culture }+0.586 * \text { Temp }))) * 100 \%
\end{gathered}
$$

\footnotetext{
${ }^{a}$ Regression coefficients after adjustment for overfitting by shrinkage (shrinkage factor $=0.83$ ), the intercept was re-estimated

$\mathrm{Cl}$ - Confidence interval; CRP - C-reactive protein; GBS - group B streptococcus
}

Table 9.2 | Prediction model for the estimation of the individual risk for neonatal sepsis 
reactive protein level and maternal temperature at admission and positive vaginal culture for Group B streptococcus were included in the final model.

After internal validation the odds ratios were 1.09 per year for maternal age, 1.01 per $\mathrm{mmol} / \mathrm{l}$ for maternal C-reactive protein level, 1.80 per ${ }^{\circ} \mathrm{Celcius}$ for maternal temperature and 2.20 for a positive Group B streptococcus culture.

The individual risk for neonatal sepsis can be estimated with the formula provided in table 9.2. Figure 9.2 shows the ROC curve of the prediction model for neonatal sepsis in women with PPROM. The area under the curve was 0.71 (95\% confidence interval $(\mathrm{Cl})=$ $0.61-0.82$ ) indicating good discriminative ability.

Figure 9.3 shows the calibration plot of the prediction model. It shows that for all risk

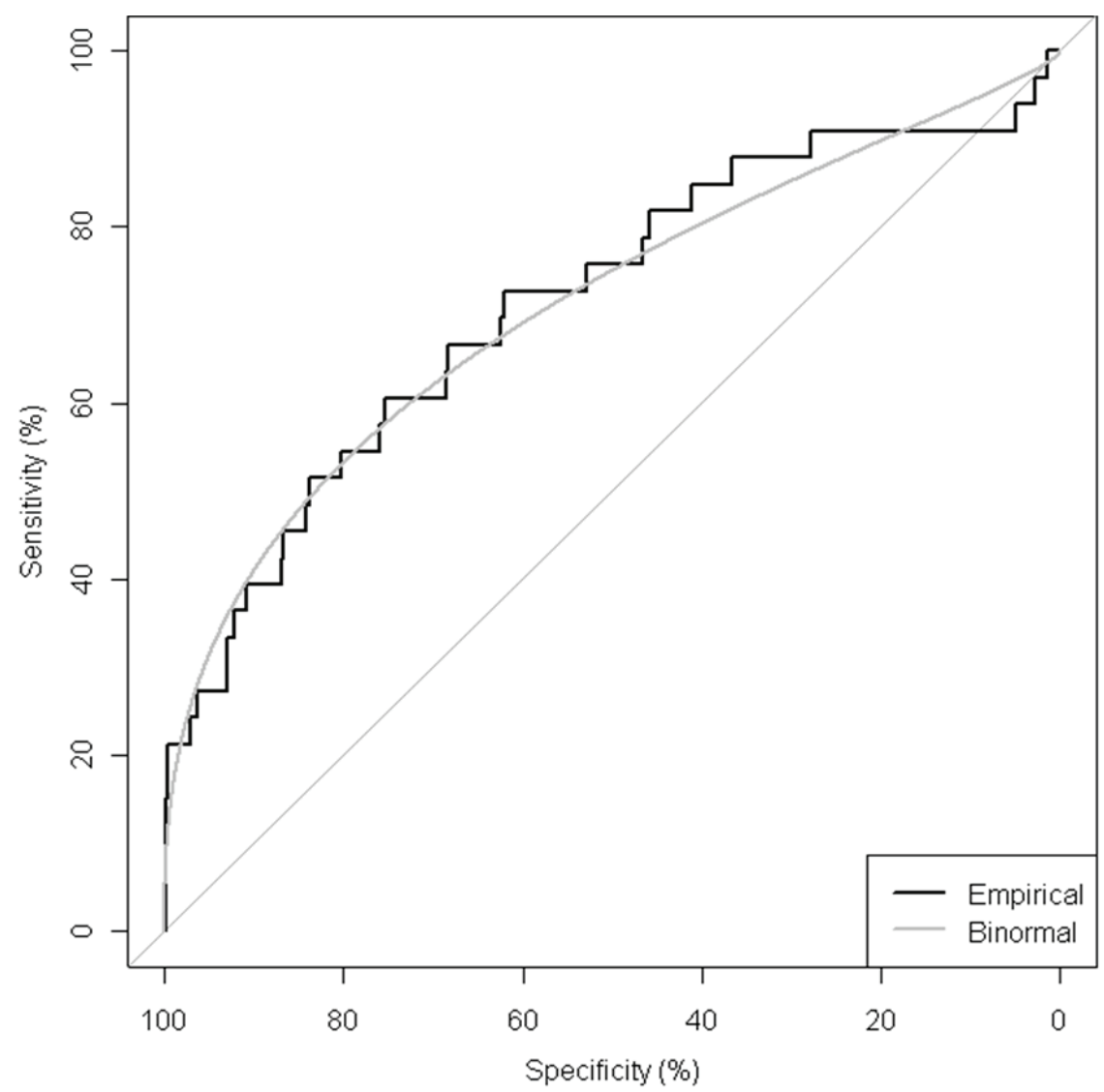

The area under the curve is $71.4 \%$ (95\% confidence interval $(\mathrm{Cl})=61.0-81.8)$. The black line shows the empirical curve, the grey line a smoothed curve based on the binormal distribution

Figure 9.2 | ROC curve of the prediction model after internal validation step 
deciles the average predicted risk is approximately the same as the observed frequency, indicating very good calibration. It does show, however, that most predicted risks are relatively low, because of the low prevalence of sepsis in our sample. Predicted risks of neonatal sepsis of over $20 \%$ are rare. Furthermore, the Hosmer and Lemeshow goodness-of-fit test yielded a P-value of 0.32 , verifying that the model is well calibrated. Overall accuracy was high, quantified by a scaled Brier score of $29.5 \%$.

\section{DISCUSSION}

In the present study we evaluated potential antenatal predictors for neonatal sepsis in women with PPROM near term. This study demonstrates that we can select predictor variables for neonatal sepsis in these women. Despite the fact that the overall incidence of neonatal sepsis in the PPROMEXIL trials was low, ${ }^{89,148}$ we were able to develop a predic-

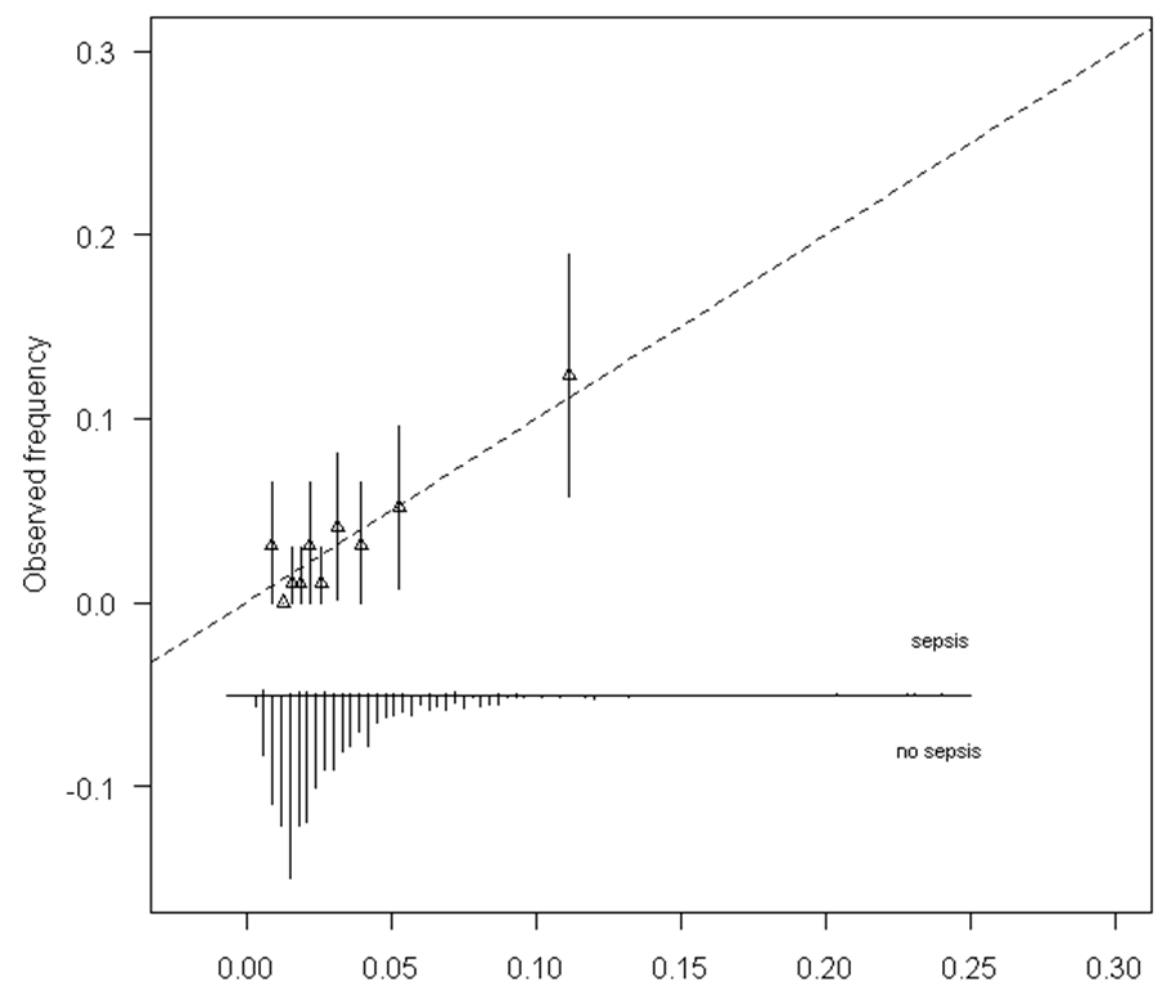

Predicted probability

Calibration plot showing observed frequency versus predicted probability for deciles of predicted risk.

Figure 9.3 | Calibration plot of the model 
tion model based on four predictors, i.e. maternal age, maternal C-reactive protein level, maternal temperature and a positive Group B streptococcus culture. The model had a good discriminative ability as well as a good calibration and overall fit of the model. Still the most predicted risks for neonatal sepsis were low and a predicted risk of more than $20 \%$ was rare. However, based on this model, one can individualise a woman's risk. Based on this individualised risk a decision can be made whether or not to monitor the woman more closely and/or induce labour.

Our study has several limitations. First, we imputed missing variables because omission of patients with missing variables could lead to a loss in statistical power. ${ }^{158-160}$ Although there are no guidelines about the maximum percentage of missing data which can be imputed, we decided to leave out erythrocyte sedimentation rate (ESR) and maternal education level because we considered the proportion of missing data of these variables as too high (33\% and $44 \%$, respectively). Since there is always an uncertainty introduced by imputing missing values, regression estimates for these variables could be unstable, and the probability of biased estimates is higher than would be the case if (many) more values would be observed. By doing this we missed out the possible predictive capacity of these variables.

Conversely, maternal CRP level and maternal temperature, which were both included in the model, where amongst the 13 potential predictors the two with the largest proportion missing data (26\% and 29\%, respectively). Table 9.1, however, shows that before imputation the distribution of these predictors were already significantly different between newborns with and without neonatal sepsis. To estimate the effect of our imputation we performed a sensitivity analysis for the complete non-imputed sample $(n=564)$. This analysis identified the same predictors with a similar effect size.

Second, we had only five events per predictor while 10 events per predictor variable is recommended. ${ }^{157}$ This might have influenced the validity of the model. We considered 13 variables as potential predictors, and by including only three in the prediction model $\left(N_{\text {cases }}=33\right.$, ten events per variable, maximum of three variables in model) would, in our opinion, have missed the predictive capacity of the other variables.

Contrary to common practice, we made no use of univariable preselection of predictor variables. Often, if a p-value of a potential predictor in univariable analysis is less than a certain predefined value, that predictor is considered for multivariable analysis. This is a common error made in model building, because one may wrongly reject predictors that are of importance in multivariable analysis. ${ }^{164}$ We chose to consider all potential predictors for multivariable analysis, given enough observed values, and use backward stepwise elimination to choose the most important predictors in these data.

Third, developing a prediction model as a secondary analysis from data of a RCT might to 
some extent limit the generalizability of the results to the whole population. Therefore external validation is needed to confirm or refute our findings.

From the four identified factors, maternal C-reactive protein level, maternal temperature and positive Group B streptococcus culture seem to have a pathophysiological explanation, whereas the increased risk of increasing maternal age is more difficult to explain. $A$ systematic review on the accuracy of CRP in women with PPROM by Van de Laar et al. could not identify any paper that had studied the accuracy of CRP in predicting neonatal sepsis. ${ }^{165}$ A more recent retrospective cohort study in 299 women with PPROM between $26^{+0}$ and $41^{+5}$ weeks showed a low predictive capacity of CRP in the prediction of neonatal sepsis (area under the ROC curve of 0.61). ${ }^{166}$ Our findings are, however, in contrast with this latter publication. This can possibly be explained by the fact that we incorporated the CRP level in the prediction model and our median gestational age at PPROM was lower $\left(35^{+3}\right.$ weeks versus $37^{+3}$ weeks). A positive vaginal culture for either Group-B streptococcus (GBS) or any species were both predictors for neonatal sepsis. Because the predictive value of both characteristics were strongly correlated, we decided to include GBS positive culture in the model, because GBS is one of the most common micro-organism causing neonatal sepsis. ${ }^{167}$ A recent Canadian study, however, showed a significant reduction in GBS as a cause for early onset sepsis over time and a trend towards an increase in other microorganisms Unfortunately, we were not able to extract data for specific microorganisms other than GBS from the database. We could therefore not use for example coagulase-negative Staphylococcus or Escheria coli as an individual bacteria for the prediction of neonatal sepsis.

In both the PPROMEXIL trials, and in the meta-analysis performed after that studies, we observed no benefit from immediate delivery in the prevention of neonatal sepsis. In view of the prognosticators that we identified, one can hypothesize that immediate delivery, maybe after antibiotic prophylaxis, might be useful in women with a high risk profile. As our studies is hypothesis generating, this should be assessed in future studies, of which the on-going Australian led PROMT study is the most important one. ${ }^{128}$

Indeed, external validation is needed to confirm the model's ability in other populations or settings to identify women with an increased risk in whom labour is advised to be induced and women with a low risk in whom the risks of complication due to prematurity outweighs the risk of neonatal sepsis and were expectant management is the best treatment strategy.

Furthermore we have planned to investigate interaction between biomarkers and the treatment allocation in the PPROMEXIL trials ${ }^{89,148}$ in the near future. 


\section{CONCLUSION}

We developed a prediction model in women with near term PPROM for the risk of neonatal sepsis, which has a good discriminative ability as well as a good calibration and overall validity. After external validation, this prediction model might be used to decide in which women labour should be induced. 



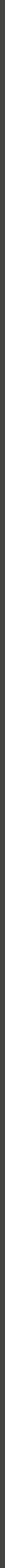




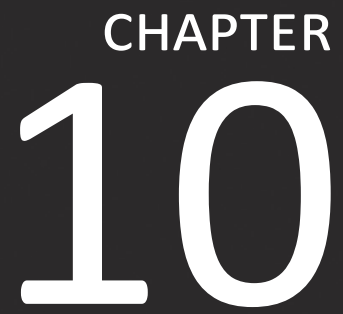

\section{ECONOMIC ANALYSIS COMPARING INDUCTION OF LABOUR AND EXPECTANT MANAGEMENT IN} WOMEN WITH PRETERM PRELABOUR RUPTURE OF

\section{MEMBRANES BETWEEN 34 AND 37 WEEKS (PPROMEXIL TRIAL)}

Sylvia MC Vijgen, David P van der Ham, Brent C Opmeer, Denise Bijlenga, Johannes J van Beek, Kitty WM Bloemenkamp, Jan B Derks, Mariët Groenewout,

Michael M Kars, Simone Kuppens, Gerald Mantel, Jan FM Molkenboer,

Antonius LM Mulder, Jan G Nijhuis, Paula JM Pernet, Martina Porath, Mallory Woiski, Martin JN Weinans, Wim J van Wijngaarden, Hajo IJ Wildschut, Bertina MC Akerboom, Marko Sikkema, Ben WJ Mol, Christine Willekes for the PPROMEXIL study group.

Submitted 


\section{ABSTRACT}

\section{Objective}

The choice for induction of labour or expectant management in women with preterm prelabour rupture of membranes (PPROM) is an important clinical problem. In the current study we compared costs associated with these strategies.

\section{STUDY DESIGN}

We performed an economic analysis alongside a randomised trial in which women with PPROM near term who were not in labour 24 hours after PPROM, had been randomly allocated to either induction of labour or expectant management, with neonatal sepsis as primary outcome. The economic analysis was done from a health care provider perspective, using a bottom-up approach to estimate resource utilisation, valued with unit-costs reflecting actual costs. All costs are presented in 2009 Euros.

\section{RESULTS}

Induction of labour did not significantly reduce the probability of neonatal sepsis (2.6\% versus $4.1 \%$, relative risk 0.64 (95\% confidence interval $[\mathrm{Cl}] 0.25$ to 1.6$)$ ). Mean costs per woman were $€ 8,094$ for induction and $€ 7,340$ for expectant management (difference $€ 754 ; 95 \% \mathrm{Cl}-335$ to 1,802). This difference predominantly originated in the postpartum period, where the mean costs were $€ 5,669$ for induction versus $€ 4,801$ for expectant management. Delivery costs were higher in women allocated to induction than in women allocated to expectant management ( $€ 1,777$ versus $€ 1,153$ per woman). Ante partum costs in the expectant management group were higher because of longer ante partum maternal stays in hospital.

\section{CONCLUSION}

In women with pregnancies complicated by PPROM near term, induction of labour does not reduce neonatal sepsis, but increases medical costs. 


\section{INTRODUCTION}

The estimated incidence of preterm prelabour rupture of the membranes (PPROM) between 34 and 37 weeks of gestation is 1.5\%, which corresponds to about 3,000 pregnancies per year in the Netherlands. PPROM is an important clinical problem, and a dilemma for both patient and gynaecologist: while awaiting spontaneous labour may lead to an increase in infectious disease and stillbirth for both mother and child, induction of labour may lead to preterm birth with an increase in neonatal morbidity (e.g., respiratory distress syndrome (RDS)) and a possible rise in the number of instrumental deliveries. ${ }^{117}$ Guidelines concerning this clinical dilemma are not straightforward. The ACOG guideline recommends induction of labour if PPROM occurs at or beyond 34 weeks of gestation. ${ }^{15}$ The British RCOG guideline states that delivery should be considered at 34 weeks of gestation. ${ }^{16}$ The Dutch NVOG guideline advised, until the results of our trial were known, expected management until 35 gestational weeks (if there are no maternal or fetal indications for immediate delivery), while labour may be induced from 35 weeks onwards, and induction of labour is advocated beyond 37 weeks of gestational age. ${ }^{5}$

In view of this lack of consensus on the management of women with PPROM between 34 and 37 weeks, we recently performed a randomised clinical trial on the subject, named the Preterm Prelabour Rupture Of the Membranes Expectant management or Induction of Labour study (PPROMEXIL, number ISRCTN29313500). ${ }^{27}$ In this study, we found that in pregnancies complicated by PPROM between 34 and 37 weeks of gestation, induction of labour did not reduce the incidence of neonatal sepsis (RR $0.64,[95 \% \mathrm{Cl} 0.25$ to 1.6]), but increased the risk of hypoglycaemia (RR 2.2 [95\% Cl 1.4 to 3.5]) and hyperbilirubinaemia (RR 1.5 [95\%Cl 1.1 to 1.9]). ${ }^{89}$

At present, information on costs and cost-effectiveness of induction of labour and expectant management in women with PPROM between 34 and 37 weeks of gestation is lacking. Because no significant difference was found on primary outcome in the trial, our study solely reports on costs and not on cost-effectiveness. An economic analysis was performed alongside the PPROMEXIL trial, comparing the costs generated by induction of labour (IoL) with the costs of expectant management (EM) in pregnancies complicated by PPROM.

\section{METHODS}

\section{TRIAL DESIGN}

Full details of the PPROMEXIL trial were reported previously. ${ }^{27}$ In short, the study was a multicentre, parallel, open labelled randomised controlled trial in the Netherlands, in 
which 60 out of 90 hospitals (eight academic and 52 nonacademic) in The Netherlands participated. Women with a singleton or twin pregnancy presenting with preterm prelabour rupture of membranes (PPROM) between $34^{+0}$ and $36^{+6}$ weeks of gestation who had not delivered within 24 hours after rupture of membranes were allocated to either loL ( $n=266)$ or $\operatorname{EM~(n=266).~}$

Induction of labour or elective caesarean section was planned within 24 hours after randomisation. The method of induction was according to local policy. Women randomised for expectant management were treated according to local policy. This was either in an outpatient or inpatient setting. If a patient in this group reached $37^{+0}$ weeks of gestation age, induction of labour was performed according to local policy.

The primary outcome measure used in the clinical trial was proven or suspected neonatal sepsis, defined as a positive blood or liquor culture or a combination of clinical symptoms (apnoea, temperature instability, lethargy, feeding intolerance, respiratory distress, haemodynamic instability, positive surface culture or rise in C-reactive protein $>20 \mathrm{mg} / \mathrm{l}$ ) in combination with antibiotic treatment during admission. ${ }^{121-123}$

\section{ECONOMIC EVALUATION}

As mentioned before we performed a cost analysis to compare costs of both strategies. We used a health care provider perspective, in which direct medical costs are included. ${ }^{169}$ We differentiated between three phases of the clinical process in which costs were generated: ante partum costs (between randomisation and onset of labour), delivery costs, and postpartum costs (between childbirth and hospital discharge). No discounting was applied because all costs occurred within one year.

Costs are calculated as the product of resource use and unit costs. Resource use during the study period was documented in Case Report Forms (CRF). The following resource items were collected: maternal and neonatal admissions, method of delivery, type of induction, outpatient visits, medication, maternal laboratory tests, neonatal monitoring. Maternal admissions were differentiated into three levels of care (intensive, medium, or ward). Neonatal admissions were divided into four levels of care (intensive, high, medium, or ward). Ward admissions of newborns were not calculated as additional costs as these were assumed to be already incorporated in costs of maternal ward admissions. Use of the labour room was calculated as hours between admission to labour room and birth plus one hour extra for extended recovery care. As induction of labour takes place inside the labour room, we expected that use of the labour room would be higher in the induction group due to time needed for induction. In case a caesarean section was performed, use of the operating room (in hours) was estimated as well.

Unit cost estimates were based on several sources: top-down calculations provided by 
the financial departments in one participating academic and one participating general hospital (for maternal and neonatal admissions to ward, medical care, obstetric high care, (N)ICU and neonatal monitoring), bottom-up calculation (one hour use of the labour room and operating theatre), Dutch standardized prices (visits to primary health care providers and outpatient visits) ${ }^{170}$ and market prices (medication). ${ }^{171}$ In table 10.1, unit costs together with valuation methods and sources are presented. All unit costs were expressed in 2009 Euros using the consumer pricing index. ${ }^{172}$ Top-down unit costs associated with vaginal delivery did not take into account differences in use of the labour room. Therefore we also estimated unit costs associated with one hour of labour room

\begin{tabular}{llcl} 
Medical costs & Unit & $\begin{array}{l}\text { Unit cost } \\
\text { (euro) }\end{array}$ & Valuation method (source) \\
\hline $\begin{array}{l}\text { Admission mother* } \\
\text { Hospital stay - ward }\end{array}$ & day & 359 & top-down calculation \\
\hline Hospital stay - medium care & day & 546 & top-down calculation \\
\hline Hospital-stay - intensive care & day & 1742 & top-down calculation \\
\hline $\begin{array}{l}\text { Admission child* } \\
\text { Hospital stay - medium care }\end{array}$ & day & 546 & top-down calculation \\
\hline Hospital stay - high care & day & 1462 & top-down calculation \\
\hline Hospital-stay - NICU & day & 1514 & top-down calculation
\end{tabular}

\begin{tabular}{|llll|}
\hline Specialist care & hour & 72 & Dutch costing guidelines \\
\hline Outpatient visit* & visit & 85 & top-down calculation \\
\hline Midwive & hour & 35 & Dutch costing guidelines \\
\hline General practitioner & visit & 22 & Dutch costing guidelines \\
\hline Paramedical & visit & 25 & Dutch costing guidelines \\
\hline Home care & hour & 33 & Dutch costing guidelines \\
\hline
\end{tabular}

\begin{tabular}{llcl}
\hline Induction methods $^{\#}$ & gift & 16 & pharmacotherapeutic website \\
\hline Medication $^{\#}$ & dose per day & 7 & pharmacotherapeutic website \\
\hline Analgesics during labour & procedure & 167 & top-down calculation \\
\hline Neonatal monitoring & procedure & 39 & top-down calculation \\
\hline Operation room* & hour & 145 & bottom-up calculation \\
\hline Labour room* & hour & 84 & bottom-up calculation \\
\hline Laboratory test & procedure & 2 & tariff \\
\hline
\end{tabular}

* the mean of the unit cost for an academic hospital and for a general hospital is presented \# the mean of several methods/medications is presented

Table 10.1 | Cost-analyses: units of resource use, unit costs and valuation 
use, and incorporated this differentiation in the analyses. In case a caesarean section was performed, hours in the operating room were estimated as well.

\section{ANALYSES}

Group differences in volumes of resource use were tested by using the nonparametric Mann-Whitney test, because these volumes are never normally distributed. Resource use per patient was multiplied by unit costs, and total costs per patient were estimated. Mean and median total costs per woman were estimated, and differences in total costs between study groups were tested using the nonparametric Mann-Whitney test. The 95\% confidence intervals around the differences in mean (sub)total costs were determined by bootstrapping. A subgroup analysis of gestational age at randomisation was performed, dividing three subgroups $A D 34^{+0}$ to $34^{+6}$ weeks, $A D 35^{+0}$ to $35^{+6}$ weeks and $A D 36^{+0}$ to $36^{+6}$ weeks.

Statistical, economic and simulation analyses were performed using SPSS software (version 16.0) and Microsoft Excel.

\section{RESULTS}

Between May $1^{\text {st }} 2007$ and September $9^{\text {th }} 2009$ a total of 776 women were asked to participate in the PPROMEXIL trial, of which 536 women (69\%) gave informed consent. Four had to be excluded after randomisation, due to violation of protocol. We allocated 266 women to IoL and 266 to EM. Of the remaining 240 women, 13 (5.4\%) did not give informed consent because they preferred induction of labour, 194 (81\%) women preferred expectant management and 33 (13.8\%) women did not want to participate in this study at all. ${ }^{89}$ For 528 women, sufficient data were available to be included in the economic analysis. Average volumes of resource utilisation, total costs as well as average costs per woman for each study group are presented in table 10.2.

In the ante partum phase, women in the EM group were found to have longer hospital stays for maternal ward care (IoL: 2.5 days versus EM: 5.0 days, $\mathrm{p}<0.05$ ) and more scheduled outpatient visits (IoL: 1.8 visits versus EM: 4.9 visits, $\mathrm{p}<0.05$ ). During delivery, differences were seen in mean number of hours in the labour room for spontaneous deliveries (IoL: 14.8 hours versus EM: 6.9 hours, $p<0.05$ ) and in mean numbers of hours in the labour room and operation theatre for caesarean sections (IoL: 27.6 hours versus EM: 18.3 hours, $p<0.05)$. Furthermore, in the loL-group more children were admitted to medium care (64\% versus 54\%) for a longer time period (IoL: 10.2 days versus EM: 8.9 days, $\mathrm{p}<0.05)$.

A summary of the mean and median total costs per woman is provided in table 10.3. In 
the ante partum period total costs per woman appeared to be higher in the EM-group, mainly due to longer duration of maternal admissions (difference: -€738;95\% Cl: -€967 to $-€ 535)$. On the other hand in the loL-group women appeared to generate more costs during delivery than women in the expectant group (difference: $€ 624 ; 95 \% \mathrm{Cl}$ : $€ 261$ to $€ 1,006)$, due to a longer duration in the labour room because of induction of labour. Until

\begin{tabular}{|c|c|c|c|c|c|}
\hline & \multicolumn{2}{|c|}{$\begin{array}{l}\text { Induction of labour } \\
(\mathrm{N}=266)\end{array}$} & \multicolumn{2}{|c|}{$\begin{array}{l}\text { Expectant manage- } \\
\text { ment }(\mathrm{N}=262)\end{array}$} & \multirow[b]{2}{*}{$\begin{array}{l}\text { Difference in } \\
\text { mean cost }\end{array}$} \\
\hline & Mean & Median (IQR) & Mean & $\begin{array}{l}\text { Median } \\
\text { (IQR) }\end{array}$ & \\
\hline $\begin{array}{l}\text { Maternal admission } \\
\text { and home-care }\end{array}$ & 632 & $\begin{array}{c}435 \\
(161-720)\end{array}$ & 1,327 & $\begin{array}{c}739 \\
(345-1,561)\end{array}$ & -695 \\
\hline Outpatient visits & 14 & $\begin{array}{c}0 \\
(0-0)\end{array}$ & 56 & $0(0-0)$ & -42 \\
\hline Laboratory tests & 2 & $\begin{array}{c}2 \\
(0-2)\end{array}$ & 3 & $\begin{array}{c}2 \\
(0-2)\end{array}$ & -1 \\
\hline Total antepartum & 648 & $\begin{array}{c}448 \\
(174-721)\end{array}$ & 1,386 & $\begin{array}{c}756 \\
(412-1,602)\end{array}$ & -738 \\
\hline $\begin{array}{l}\text { Admission because of } \\
\text { labour }\end{array}$ & 257 & $\begin{array}{c}298 \\
(0-325)\end{array}$ & 270 & $\begin{array}{c}298 \\
(0-325)\end{array}$ & -13 \\
\hline Induction material & 45 & $\begin{array}{c}0 \\
(0-94)\end{array}$ & 13 & $\begin{array}{c}0 \\
(0-0)\end{array}$ & 32 \\
\hline $\begin{array}{l}\text { Medication during } \\
\text { labour }\end{array}$ & 88 & $\begin{array}{c}32 \\
(0-167)\end{array}$ & 69 & $\begin{array}{c}32 \\
(0-167)\end{array}$ & 19 \\
\hline Mode of delivery & 1,387 & $\begin{array}{c}747 \\
(415-1,245)\end{array}$ & 801 & $\begin{array}{c}415 \\
(168-814)\end{array}$ & 586 \\
\hline Total delivery & 1,777 & $\begin{array}{c}1,063 \\
(630-1,751)\end{array}$ & 1,153 & $\begin{array}{c}750 \\
(458-1,227)\end{array}$ & 624 \\
\hline $\begin{array}{l}\text { Maternal admission } \\
\text { and home-care }\end{array}$ & 1,259 & $\begin{array}{c}1,192 \\
(596-1,680)\end{array}$ & 1,243 & $\begin{array}{c}1,192 \\
(840-1,680)\end{array}$ & 16 \\
\hline Neonatal admission & 4,399 & $\begin{array}{c}2,287 \\
(0-5,362)\end{array}$ & 3,547 & $\begin{array}{c}1,300 \\
(0-4,225)\end{array}$ & 852 \\
\hline $\begin{array}{l}\text { Neonatal monitoring+ } \\
\text { lab tests }\end{array}$ & 11 & $\begin{array}{c}2 \\
(2-4)\end{array}$ & 11 & $\begin{array}{c}2 \\
(0-4)\end{array}$ & 0 \\
\hline Total postpartum & 5,669 & $\begin{array}{c}3,783 \\
(1,490-6,869)\end{array}$ & 4,801 & $\begin{array}{c}2,494 \\
(894-5,684)\end{array}$ & 868 \\
\hline Total cost $(95 \% \mathrm{Cl})^{\mathrm{b}}$ & 8,094 & $(1,083-25,932)$ & 7,340 & $(670-30,874)$ & $\begin{array}{c}754 \\
\text { (-335 to } 1,802)\end{array}$ \\
\hline
\end{tabular}

a Induction minus expectant management

${ }^{b}$ non-parametric confidence interval based on 1000 bootstrap replications

Table 10.3 | Comparison of costs between induction of labour and expectant management 


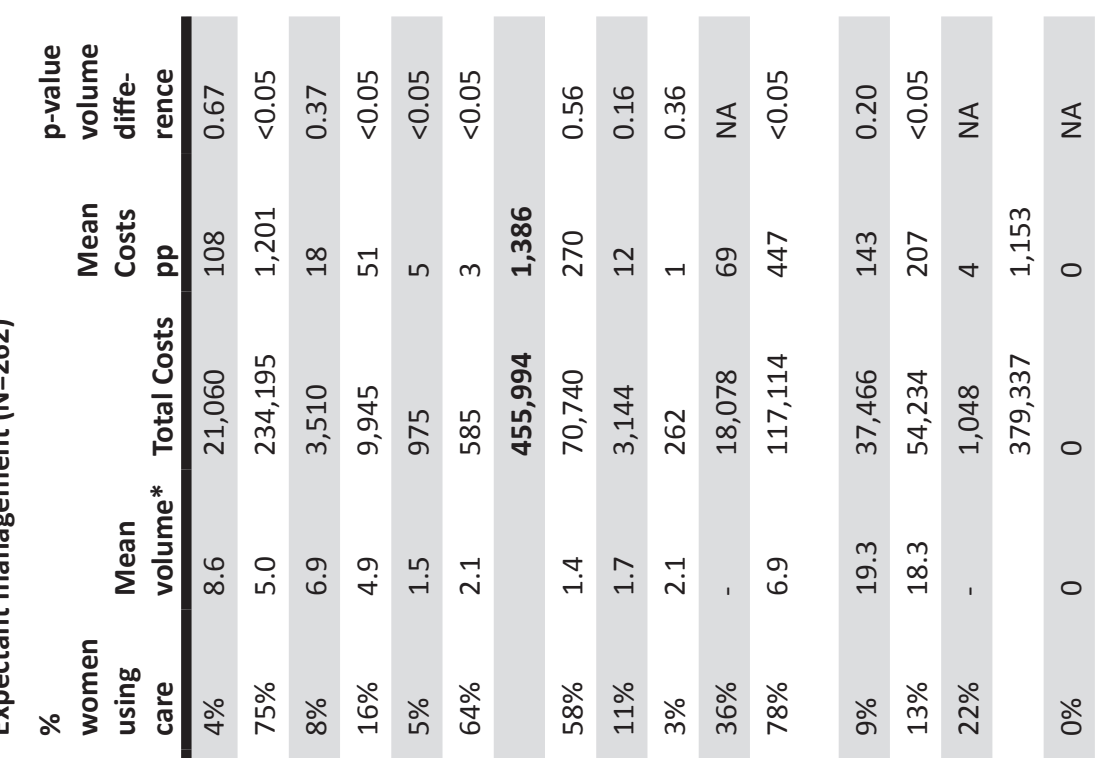

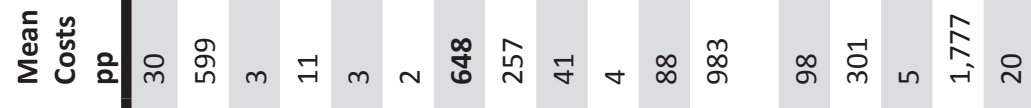

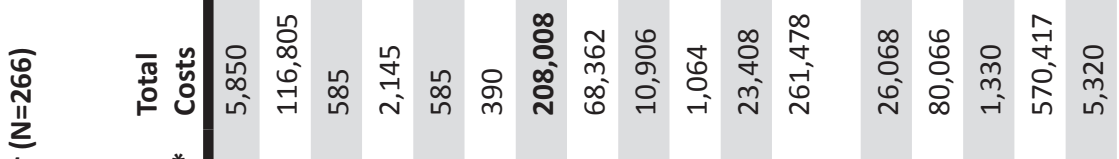
ํㅗㅇ

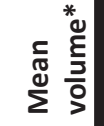

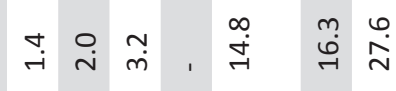

ํㅜㄴ

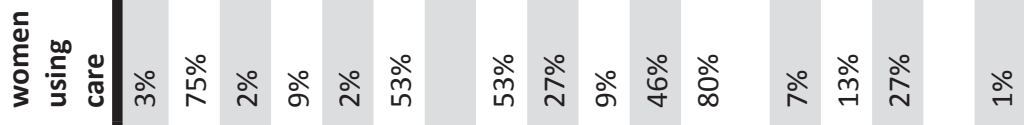

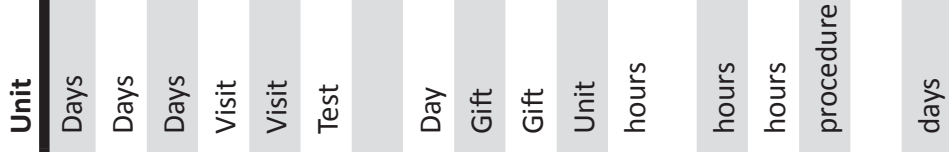

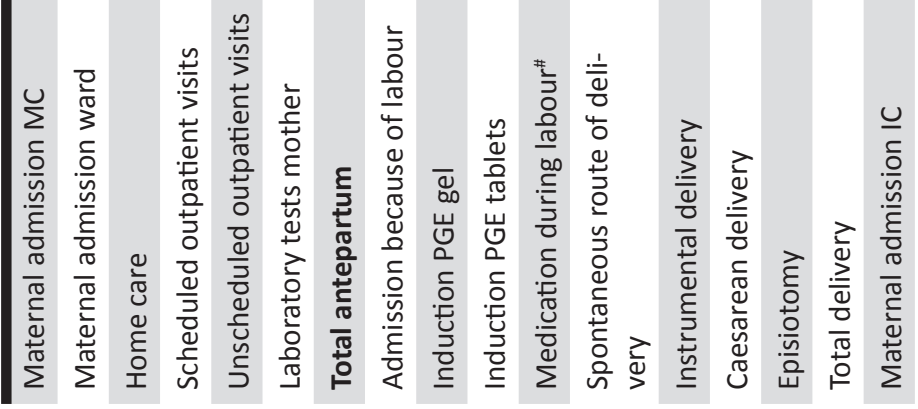




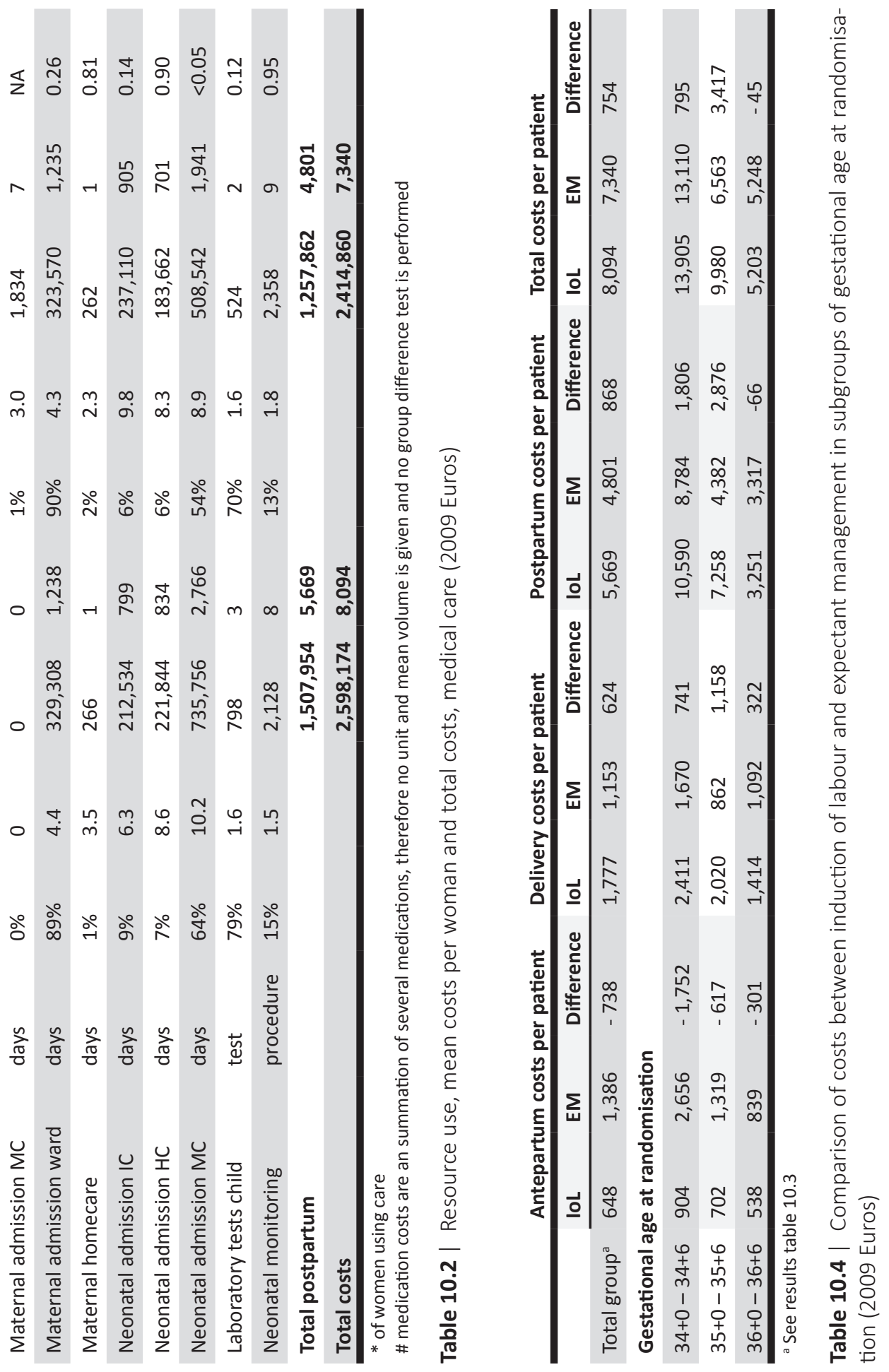


6 weeks postpartum women in the loL-group again generated higher costs than those in the EM-group (difference: $€ 868 ; 95 \% \mathrm{Cl}$ : -€41 to €1,929), mainly because of more and longer duration of neonatal medium care admissions. Overall, mean costs per patient were $€ 8,094$ (95\% Cl: €1,083 to €25,932) for loL and €7,340 (95\% Cl: €627 to €30,874) in the EM-group (difference $€ 754$ : 95\%Cl: - $€ 335$ to $€ 1,802$ ).

The results of the subgroup analysis dividing the patients in three groups according to gestational age at randomisation are presented in table 10.4. As can be seen from this table the differences in total costs per woman are almost zero for women between 36 and 37 weeks of pregnancy (-€45). On the other hand loL generates much more costs in women with gestational age between 35 and 36 weeks $(€ 3,417)$. Although patients with gestational age between 34 and 35 weeks generate more mean costs per person, the difference between IoL and EM is comparable with the total group difference ( $€ 795$ versus $€ 754)$.

\section{DisCUSSION}

In this study, we report that in women who's pregnancy is complicated by PPROM between 34 and 37 weeks of gestational age, EM generated less costs than loL, although the difference was not statistically significant (€ 754: 95\% Cl: - $€ 335$ to $€ 1,802$ ). The difference was mainly resulting from more and longer neonatal admissions after loL, which was either due to the increased risk of hypoglycaemia (RR 2.2 [95\% Cl 1.4 to 3.5]) and hyperbilirubinaemia (RR 1.5 [95\% Cl 1.1 to 1.9]) or the effects of prematurity in general in the loL-group. In the trial no differences were found in maternal morbidity between groups. As can be seen from our results, postpartum maternal admission costs were comparable as well.

This economic analysis was performed from a health care instead of the societal perspective. ${ }^{169,170}$ This was motivated as we did not anticipate any large differences in patient or productivity loss costs. In a previous economic study comparing induction of labour with expectant monitoring in women with pregnancy induced hypertension (PIH) or preeclampsia (PE) beyond 36 weeks of gestation, we found no impact of non-medical costs on the final outcome. ${ }^{173}$

A limitation of our study might be the short time horizon we used, from ante partum hospital admission until post partum hospital discharge, a strategy that was based on the available trial based data to estimate both clinical outcomes and costs. For future research, extrapolation of our data to a long term time horizon using a decision model is recommended. Observed cost differences were apparently not statistically significant when we used the boot strap technique. However, as the most important cost drivers 
behind these differences, such as neonatal medium care admission was statistically significant, thus providing a reliable fundament under the reported cost difference.

Subgroup analysis of different gestational ages at randomisation showed no costs difference between IoL and EM for women with gestational age between 36 and 37 weeks. The costs difference for women with gestational age between 35 and 36 weeks appeared to be much higher. This indicates that loL does not generate more costs than EM for women with PPROM beyond 36 weeks of pregnancy.

Overall the large multicentre PPROMEXIL trial demonstrated that women with preterm prelabour rupture of membranes between 34 and 37 weeks of gestational age can be treated expectantly without an increased risk of neonatal sepsis, less neonatal morbidity due to hyperbilirubinaemia or hypoglycaemia and equal maternal morbidity ${ }^{89}$ and maybe even at lower costs. Due to a lower than expected incidence of neonatal sepsis more research is needed to support both clinical and economic findings. In a recent updated meta-analysis, we could report on 1,090 neonates for the endpoint neonatal sepsis, 1,428 neonates for the endpoint RDS, and 1,417 women for the endpoint Caesarean Section. The relative risks of IoL as compared to EM were 0.85 ( $95 \% \mathrm{Cl} 0.48$ to 1.5) for neonatal sepsis, $1.04(95 \% \mathrm{Cl} 0.88$ to 1.3$)$ for RDS and 1.1 (95\% Cl 0.88 to 1.4$)$ for Caesarean section. ${ }^{148}$ These data confirm the assumption of this economic analysis that both policies of IoL and EM result in similar neonatal and maternal outcomes. Currently, the Australian lead global PROMT trial is recruiting over 1,800 women for exactly the same dilemma. ${ }^{128}$ This larger trial will also include an economic analysis alongside the trial. Apart from more accurate estimates on the rates of neonatal sepsis and other neonatal outcomes, that will be provided by PROMT, the pressing issue in loL of women with ruptured membranes near term is the risk of long term neonatal damage in case of preterm delivery. A recent meta-analysis from our group on this issue, ${ }^{174}$ as well as large cohort study from Liverpoo ${ }^{175}$ indicated a considerable increased risk of pathology in case of late preterm delivery as compared to term delivery. On the other hand, after PPROM, the fetal risk of umbilical cord compression of prolapse might be increased, resulting in asphyxia or even still birth. As these issues have not been addressed with adequate data, definite conclusions cannot be drawn.

In summary, we found in the PPROMEXIL trial that induction of labour did not reduce the risk of neonatal sepsis, whereas costs associated with this strategy are probably higher. While data on stillbirth risk after PPROM and long term outcome after late preterm delivery have to be awaited, it is clear that children born just before term do worse in childhood than those born at 37 weeks. In view of these data, and until additional data are available, we recommend expectant monitoring until 37 weeks in women with PPROM near term. 


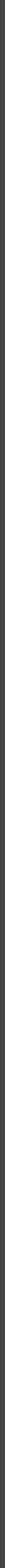




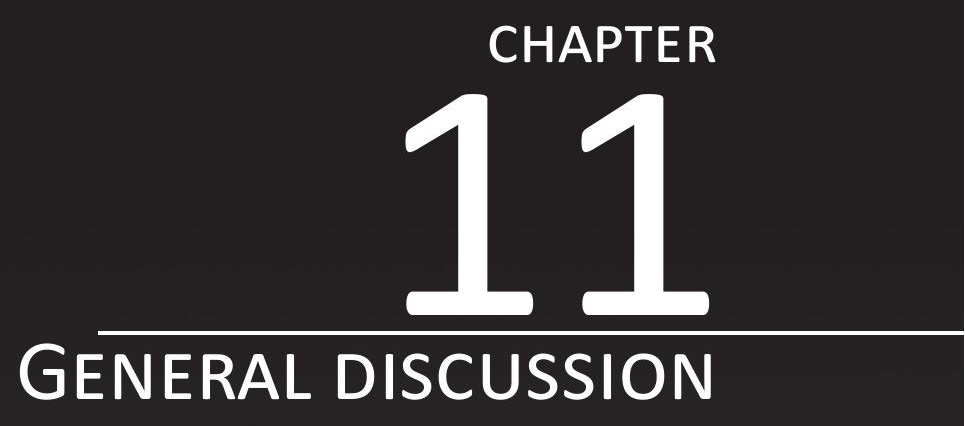




\section{INTRODUCTION}

Prelabour rupture of membranes (PROM) is a frequently encountered obstetrical problem. It complicates approximately $10 \%$ of all pregnancies and is associated with chorioamnionitis and perinatal and maternal morbidity. ${ }^{1,2}$ Preterm PROM complicates $1-5 \%$ of all pregnancies and accounts for $30-40 \%$ of all preterm deliveries. ${ }^{30-32}$ PPROM is associated with several clinical problems such as chorioamnionitis, ascending infection, neonatal infection or sepsis, prematurity, abruption placentae, cord prolapse and possibly increased risk of stillbirth. 2,7,8 In The Netherlands, the pregnancy of approximately 3,000 women is annually complicated by PPROM.

Current recommendations on the treatment of late preterm rupture of membranes around the world are "consensus" based, as there is a lack of high quality evidence. Different opinions have led to different recommendations.,15,16 The American ACOG guideline recommends induction of labour if PPROM occurs at or beyond $34^{+0}$ weeks of gestation. ${ }^{15}$ The British RCOG guideline states that delivery should be considered at $34^{+0}$ weeks of gestation and recommends that women with PPROM who are managed expectantly beyond 34 weeks of gestation should be counselled about the increased risk of chorioamnionitis and the presumed decreased risk of neonatal respiratory problems, admission for neonatal intensive care and caesarean section. ${ }^{16}$ Canadian and Australian surveys notice the lack of consensus on management in women with PPROM between $34^{+0}$ and $36^{+0}$ weeks. ${ }^{17,18}$ The Dutch guideline advises expectant management until $35^{+0}$ gestational weeks, recommends to discuss induction of labour with the patient from $35^{+0}$ weeks onwards, whereas induction of labour is advocated beyond $37^{+0}$ weeks gestational age. ${ }^{5}$

The aim of this thesis was to improve knowledge on the management for women with late preterm prelabour rupture of membranes. We conducted a multicentre randomised controlled clinical trial under the acronym PPROMEXIL (Induction of Labour versus Expectant Management in women with Preterm Prelabour Rupture of Membranes between 34 and 37 weeks). The results of the PPROMEXIL trials are presented in Chapter 6 and Chapter 7. These studies have provided an evidence-base for the management of women with PPROM near term.

In women whose pregnancy is complicated by late preterm PROM, induction of labour does not substantially improve the outcome for either the mother or her child compared to expectant management. These findings were confirmed by data from the Dutch Perinatal Register (PRN), which we presented in Chapter 8. Until the results of a larger similar trial (PROMT trial) are known,, ${ }^{128}$ we recommend expectant management in women with late preterm PROM, except for women who have a positive culture for GBS, as we showed in a prediction model in Chapter 9. 


\section{DIAGNOSTIC TEST FOR RUPTURE OF MEMBRANES}

Chapter 2 and Chapter 3 describe the use of diagnostic test for rupture of the fetal membranes. Only a few studies focused on membrane rupture in equivocal cases. ${ }^{45,58,70} \mathrm{An}$ updated review (Chapter 3) did not yield new insights in these specific cases. The question remains whether or not there is a need for (new) diagnostic tests, especially in relation to the results of the PPROMEXIL trials.

From a clinical point of view there seems to be a need for gynaecologists to be supported in the diagnosis of ruptured membranes. ${ }^{81}$ Furthermore little is known of the clinical outcome in women with equivocal ruptured membranes. Similar to the way fetal fibronectin is used to predict preterm labour, i.e. to rule out preterm delivery within 7 days, ${ }^{176-179}$ diagnostic tests for rupture of membranes might possibly be helpful especially in case there is doubt to differentiate between watchful waiting (positive test) or normal pregnancy controls (negative test). However, randomised controlled trial are needed to evaluate if diagnostic tests like PAMG-1 and SICAM1 have these potentials. If so, one might even consider the use of these test in a first line (midwife) setting to decide whether or not to refer a patient to the hospital, which eventually can lead to reduce costs of outpatient hospital visits and probably unnecessary hospital admissions.

\section{WATCHFUL WAITING IN NEAR TERM PPROM}

As shown in Chapter 6 and Chapter 7 from the results of the PPROMEXIL trials and confirmed in Chapter 8 using data from the Perinatal Registration Netherlands watchful waiting seems to be the best policy in late preterm PROM. Although the PPROMEXIL trials doubled the number of patients on which evidence on the management of women with late preterm PROM is made, the study itself was still underpowered. In retrospect, anticipating a risk reduction of 66\% (a difference in neonatal sepsis rate of $7.5 \%$ versus $2.5 \%$ ) might have been too optimistic. However, several previous studies $20,22,24$ showed neonatal sepsis incidences up to $9.5 \%$ with EM, and we did observe an incidence near $2.5 \%$ in the loL group. Although optimistic, we feel that our hypothesized risk reduction was not unrealistic.

Furthermore, for the first PPROMEXIL trial presented in Chapter 6 we used a one-sided test for the power calculation. We considered it not plausible that IoL in women with PROM near term would increase the proportion of cases of neonatal sepsis. In retrospect, considering the results of the meta-analysis, one might question this choice of a onesided test, as several studies in the meta-analysis show an increased risk for sepsis in the

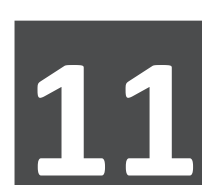


IoL group. A two-sided test would have required a sample size unfeasible in our setting, in view of the limitations set by our funding body.

Although we recommend expectant management in women with PROM near term with a negative GBS culture, this does not imply that women can be followed in a regular control schedule. In Chapter 8 we observed that "watchful waiting" under strict monitoring in women with PROM ( $>24 \mathrm{~h}$ ) reduces risk of stillbirth compared to "normal" on-going pregnancies. This means that we monitor pregnancies complicated by PROM probably more strictly. Since monitoring at home was done in only a small proportion of included women, we were not able to study the effect and possible risks of home care. Monitoring at home could reduce cost and may be preferred by women, however new studies are needed to proof safety of home care in women with near term PPROM.

\section{FUNDING LARGE CLINICAL TRIALS}

In retrospect, the lack of power was a limitation of the PPROMEXIL trial. The main reason for this lack of power was a lack of funding. The funding program allowed a period of 3 years to complete the trial. Together with the budget limitation, this limited the maximal achievable sample size to 500. If the system of funding per trial stays as it is, this will in our opinion remain one of the main limitations of future trials. Lack of funding is, however, a global problem, which was also faced by the investigators of a similar Australian Preterm Prelabour Rupture of Membranes Close to Term Trial (PROMT). ${ }^{128}$ This trial, however, is allowed more time and will recruit over just more than a decade, which is virtually impossible in the Dutch context.

Before we were certain that the PROMT trial was to be continued to its targeted sample size of 1,800, one of the main reasons to continue inclusion of the PPROMEXIL trial within a new study (Chapter 7), was to generate as much evidence on the subject as we could. This was done without any additional funding and was only possible because of the fact that the PPROMEXIL trial was one of the Dutch Consortium studies.

We believe that from global perspective generation of evidence could be planned more effectively and efficiently. Close contact between principal investigators and their funders around the world at the moment that similar trials are registered would be helpful. One could have collaborative execution of the trials under the umbrella of a prospective individual patient data meta-analysis, leaving the decision when to stop studies in such a collaborative to a Data Safety Monitoring Board overseeing all the trials. A first step to reach this aim was made in 2010 by the founding of the Global Obstetrics Network (GONet). (www.globalobstetricsnetwork.org). 
GONet was formed to link different types of (national) networks and to provide a forum for international interaction and collaboration among groups that perform clinical trials and observational studies in maternal fetal medicine and obstetrics. ${ }^{180}$ Partners of the global

\section{THE GOALS OF GONET:}

To facilitate an interface of knowledge and collaboration through the development of a database of on-going and planned studies

To define obstetrical trial/study terms/definitions and create a registry of definitions

To provide suggested tables/minimal data to be collected for different clinical problems for obstetric trials

To coordinate study protocols to facilitate and initiate prospective meta-analysis of studies and retrospective IPD meta-analysis

To identify opportunities to participate in and support current research

To identify novel research opportunities for the GONET group develop pathways for protocols to be submitted/ discussed at GONet

To determine if it is feasible to facilitate an international collaboration of funding bodies to support trials

To discuss areas of critical importance in obstetrics and identify trials/studies that need to be performed

To enhance international education and training in the design and performance of studies and clinical trials

obstetric network are the Global Alliance on the Prevention of Prematurity and Stillbirth (GAPPS), World Health Organisation (WHO) and Core Outcome Measures in Effectiveness Trials (COMET). GAPPS leads a collaborative, global effort to increase awareness and accelerate innovative research and interventions that will improve maternal, new-born and 
child health outcomes (www.gapps.org). WHO is the directing and coordinating authority for health within the United Nations system (www.who.int/en/). The COMET (Core Outcome Measures in Effectiveness Trials) Initiative brings together people interested in the development and application of agreed standardised sets of outcomes, known as 'core outcome sets'. (www.comet-initiative.org). It can be expected that GONet will lead to better studies, less duplication and more efficient use of funding. ${ }^{180}$

\section{INDIVIDUALISING RISK ASSESSMENT}

In our PPROMEXIL studies, 96\% of the 240 non-randomised women declined participation because they had the feeling that expectant management would be better for them as an individual. Individualising risk assessment is the next step in the treatment of women with late preterm PPROM. Identification of a high-risk subgroup in women with PPROM between 34 and 37 weeks could be helpful in the management of these women. C-reactive protein is an acute phase protein which had been shown to have a positive correlation with clinical and/or histological chorioamnionitis. ${ }^{92-96}$ The use of CRP as a predictor for early diagnosis of chorioamnionitis was systematically reviewed and found to be of limited use. ${ }^{97}$ In Chapter 4 we demonstrated that there is also insufficient evidence to use CRP as an predictor for neonatal sepsis .Further trials with larger populations should be conducted to determine the usefulness of CRP. This was partly done in the secondary analysis of the PPROMEXIL trial in Chapter 9, in which there seemed to be a use for CRP in the prediction of neonatal sepsis.

In Chapter 9 we showed that maternal age, maternal C-reactive protein level, maternal temperature and a positive group B streptococcus culture could be used in a prediction model to identify an increased risk of neonatal sepsis, nevertheless this prediction model has to be externally validated, before it can be used in clinical practice.

Another approach to individualize risk assessment would be to focus on the bacteria that commonly causes neonatal sepsis, namely the group B streptococcus (GBS). ${ }^{181}$ The primary pathway of GBS infection is vertical transmission of maternal genitourinary or gastrointestinal GBS colonization, which generally occurs after rupture of membranes or onset of labour. ${ }^{182-184}$ Since the time between rupture of membranes and delivery is a known risk factor for neonatal GBS sepsis ${ }^{185}$ and women who undergo expectant monitoring strategy have a longer time to delivery compared to women in whom labour is induced immediately. It could therefore be hypothesized that fetuses of women with GBS colonization might be at higher risk of neonatal sepsis when treated expectantly and therefore, they may benefit more from labour induction. In a secondary, yet unpublished, post hoc analysis of the pooled data of the PPROMEXIL trials, it seems that in women who had GBS colo- 
nization, the risk of neonatal sepsis was high after expectant management (15.2\%) and induction of labour could significantly reduce this risk (1.7\%). These data were outside the scope of the current thesis, but will be reported in upcoming publications.

These findings may even more improve the management of women with near term PPROM. After a quick GBS screenings test and based on antepartum clinical parameters the decision can be made between induction of labour, watchful waiting in an inpatient setting and watchful waiting in an outpatient setting.

\section{NEXT STEPS IN MANAGEMENT OF PPROM}

With the completion of the Australian PROMT trial within the next year, ${ }^{128}$ the amount of evidence will probably be solid enough to guide clinical practice in women with near term PROM. As we have discussed in Chapter 6 and Chapter 7, we have planned to execute an individual patient data (IPD) meta-analysis with the Australian PROMT group. This IPDmeta-analysis will hopefully also give us the possibility to study subgroups, validate prediction models and individualise risk assessments for adverse neonatal outcome.

The real burden in premature prelabour rupture of membranes lies in the midtrimester PPROM population. Although only $0.6 \%$ of pregnancies is complicated by PPROM before 27 weeks, ${ }^{186}$ midtrimester PPROM is associated with severe neonatal complications and fetal death. ${ }^{187}$ Future research should be more focussed on this high risk population.

Within the Dutch consortium for women's health and reproductivity studies we are currently running the PPROMEXIL III trial (Trial register: NTR 3492), ${ }^{188}$ In this trial woman whose pregnancy is complicated by rupture of membranes between 16 and 24 weeks of gestational are randomised between expectant management and (repeated) amnioninfusion. The primary outcome of this study is perinatal mortality.

Besides the high risk of perinatal mortality and morbidity, risks of recurrent preterm labour seems to be higher. However, little is known on the real incidence of this risk. Data from the Dutch Perinatal Registry shows that in women with a history of PPROM, the absolute risk of recurrent PPROM is low (0.7\%).In women with a history of PPROM the risk of a preterm delivery in the subsequent pregnancy is nevertheless 4.8 fold increased, compared to women with a history of a term pregnancy. In women with early PPROM $(<32$ weeks) the risk of a preterm delivery in the subsequent pregnancy is strongly increased (15 fold). ${ }^{189}$ Still the vast majority of women with a history of midtrimester PPROM will deliver at term. ${ }^{189}$ These findings together with the outcome of the PPROMEXIL III trial will contribute to a better counselling of women about the risk of the present and possible subsequent pregnancy. 


\section{DUTCH CONSORTIUM}

This thesis is one of many as a resulting of the efforts of the Dutch Consortium for women's health and reproductivity studies (www.studies-obsgyn.nl). The PPROMEXIL trial was the fourth randomised controlled trial which started within the Dutch Consortium. Since the start of the Dutch Consortium in 2005 our collaboration has addressed important clinical questions within the structure of a multicentre randomised controlled trial. Meanwhile this has led to several high impact peer reviewed publications. ${ }^{144,145,190-197}$ At present (February 2013) 32 trials in obstetrics, 12 trials in reproductive medicine, 13 trials in (uro)gynaecology and 1 trial in gynaecological oncology have been completed, while 13 obstetric, 13 reproductive medicine, 3 fetal medicine, 5 gynaecological, 10 urogynaecological and 3 gynaecological oncology studies are still on-going with another 9 new trials planned to start within the next year.

These new studies will contribute to an improvement of the body of knowledge supporting interventions that are thought to benefit women's and children's health.

Due to the fact that the PPROMEXIL trial was one of the first large multicentre trials with 60 participating hospitals across The Netherlands, we faced several (logistic) problems. One of the major problems was the time consuming Medical Ethical Committee (MEC) procedure that had to be executed on every single hospital. Before start of the trial we had a MEC approval from the Maastricht University Medical Centre (05-240). Participating hospitals had to have an additional local MEC approval. The local MEC approval took up to several months per hospital, which resulted in a consecutive start of the local hospitals. The same problem was described by Van Santvoort et al in a prospective evaluation of their multicentre PANTER trial. ${ }^{198}$ In the 18 participating hospitals in the PANTER trial it took between 35 and 361 days to obtain MEC approval. Overall took it 2 years to obtain all medical approval.

This obliged time consuming legal procedure in achieving medical ethical was recognised by the Central Committee on Research Involving Human Subjects (CCMO). The 27 medical ethical committees together with the CCMO, as an overarching committee, are responsible for the judgment of all medical research proposals in the Netherlands. Due to these time-consuming procedures the CCMO has set up a new procedure. The aim of this new procedure is to simplify the MEC approval for multicentre trials.

In short this procedure means that since March 2012, study protocols only need to be approved by the MEC allied to the centre of the principal investigator. After approval by the MEC, local investigators sign a Research Declaration Form which is then send back to the MEC of the principal investigator's centre. The MEC gives approval for participation of the local hospital. The final step is a formal consent by the Board of the local hospital. 
Although not yet all hospitals adjust to these new rules, this alteration in the MEC regulation have tremendously accelerate the local approval procedure. Another issue was the magnitude of the case report file (CRF). Initially we had made the CRF too large. For example we aimed to register all moments of blood sampling. This made the workload high and we felt that the risk of missing essential information was significant. We therefore decided in an early stage of the trial to reduce the size of the CRF without losing essential information. Lessons learned from our and other (over) ambitious CRFs (e.g. HYPITAT, DIGITAT trial) has led to a more compact, less time-consuming CRF in latter studies.

At present the Dutch Consortium has become an important initiative in Obstetrical and Gynaecological research in The Netherlands and is one of the key players around the world. Structural funding and incorporation of the consortium as part of the Dutch Society of Obstetrics and Gynaecology (NVOG) are important next steps. Where participation of as many hospitals as possible throughout The Netherlands was the first goal, that has more or less been achieved, the future goal should be a further professionalising of the consortium. With structural funding it seems feasible to setup a scientific board, with clinical researchers, methodologists, epidemiologists and biostatisticians which is supported by a full-time research office. This together with a permanent data safety monitoring board will improve the quality of future trials.

Another step which can and maybe must be made within the next year(s) is at the source of our data, the local hospitals. Due to budget limitations it is impossible to have research nurses available on all sites. Data collection and data processing is done within the capacity of the current infrastructure and budget. This however, results in incomplete datasets, which may result in imputing data in order to develop a prediction model, as we did in Chapter 9, but more seriously might lead to incorrect results. In order to improve the quality of data (collection) "Good Clinical Practice" (GCP)-certified research nurses need be available on all research sites and preferable at all time. The budget of the Dutch Consortium will be insufficient to realise this. However, as the implementation of the HYPITAT trial (induction of labour versus expectant monitoring in women with gestational hypertension or pre-eclampsia at term) clearly shows: hospitals participating in the trials have better outcome during and after the trial, compared to non-participating hospitals. ${ }^{199}$ From that perspective it is also in the importance of hospital Boards and health care insurance companies to prioritise participation in and/or funding of (clinical) research, not only from scientific point of view but also to improve quality of (female) healthcare in general. When all the above can be achieved the quality of Dutch consortium trials will reach a level in which participating in these trials will be a privilege and a quality assessment tool for all Dutch Hospitals.

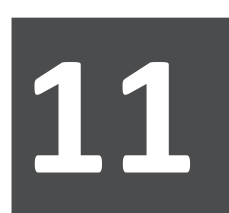




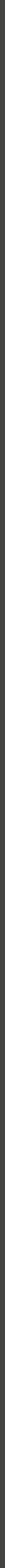




\section{SUMMARY}

\section{SAMENVATTING}




\section{SUMMARY}

Premature prelabour rupture of membranes (PPROM) occurs in approximately 3\% of all pregnancies, in $1.5 \%$ before 34 weeks of gestational age and in approximately $1.5 \%$ between 34 and 37 weeks. This means that in The Netherlands approximately 3,000 women are annually admitted to the hospital because of PPROM between 34 and 37 weeks. PPROM is associated with several clinical problems such as chorioamnionitis, ascending infections, neonatal sepsis, prematurity, abruption placentae, cord prolapse and possible risk of stillbirth.

International guidelines have different opinions on the treatment of near term prelabour rupture of membranes. Due to the lack of solid evidence treatment recommendations are based on small studies and expert opinions.

The lack of consensus and evidence on the management of prelabour rupture of membranes in late preterm women was the onset of this thesis and the Induction of Labour versus EXpectant management in women with Preterm Prelabour Rupture of Membranes between 34 and 37 weeks (PPROMEXIL) trial.

At the start of the PPROMEXIL trial we performed a prior-to-belief questionnaire among gynaecologists and residents (Chapter 1). The knowhow and beliefs on 236 (20\%) responders varied widely. Eighty two per cent of responders indicated that women were treated expectantly prior to the PPROMEXIL trial. The median estimated risk for neonatal sepsis in women with near term PROM when treated expectantly, was 5\% with a very wide range ( $0 \%$ to $70 \%$ ). This wide range of estimated outcomes was seen for all outcomes. This finding is concerning, because if assumptions of professionals are not in concordance with the real risks, the gut feeling of the professional might influence their policy more than solid evidence.

Within the Dutch Consortium an implementation study is planned to investigate the implementation of the results of several trials in daily practice.

In Chapter 2 we systematically reviewed diagnostic methods for rupture of membranes. In the vast majority of the cases a diagnosis of ruptured membranes can be made on a patient's history and visualisation of amniotic fluid loss per vaginam or with speculum examination. However, in $10 \%$ of the cases the diagnosis is unclear. In our opinion there is no need for a diagnostic test in women with clear rupture of membranes, however in women were the diagnosis is equivocal a diagnostic test is needed. We therefore systematically reviewed the literature and limited our target population to women with doubtful rupture of membranes. Without language restrictions and with a broad search strategy 
we identified over 3800 studies. After screening title and/or abstract we obtained 146 full manuscripts. We excluded 133 studies due to multiple reasons, of which inadequate test description ( $n=49$ ) and an inappropriate study population (only unequivocal rupture of membranes or amniotic fluid obtained after artificial rupture of membranes; $n=33$ ) were the most common reasons.

An expert panel scored the remaining 13 studies. Only three studies passed our predefined criteria according to STARD and QUADAS criteria. These three studies diagnosed rupture of membranes with three different methods, i.e. $\mathrm{pH}$-measurement, insulin-like growth factor binding protein-1 and alpha-fetoprotein. Due to the small studies ( 8 to 83 patients per study) and different methods we could not identify or recommend a particular test for the diagnosis of ruptured membranes.

We updated the findings of Chapter $\mathbf{2}$ in an invited review to evaluate diagnostic accuracy studies for rupture of fetal membranes as is presented in Chapter 3. A new search yielded seven new studies. Overall sample sizes of these recent studies were small and most authors used different "silver standard" reference tests. The focus of recent diagnostic studies has been on two bedside test strips: insulin-like growth factor binding protein-1 (IGFBP-1) and placental $\alpha$-microglobulin-1 (PAMG-1). Compared to nitrazine or ferning test alone IGFBP-1 and PAMG-1 are more accurate. However, compared to the conventional testing (history, ferning, nitrazine test, speculum examination and ultrasound) these tests were not better. Although none of the studies used the golden standard (amniocentesis with infusion of a dye) as a reference test, the claimed sensitivity and specificity should be interpreted with caution. However, the use of a bedside test improved the confidence of clinicians about their diagnosis. In-vitro PAMG-1 is shown to be superior to IGFBP-1 when amniotic fluid is diluted with physiological saline solution. New biomarkers such as soluble intercellular adhesion molecule-1 (sICAM-1) and Axl receptor tyrosine kinase (AxI), which are highly present in amniotic fluid and nearly absent in cervical-vaginal fluid, seem promising. However, as long as diagnostic test are not tested against the golden standard, the results of new studies will always be issue of debate.

Early detection of an intra-uterine infection and prevention of neonatal sepsis will be helpful in the management of women with PPROM. In Chapter 4 we evaluated the capacity of C-reactive protein (CRP) to predict chorioamnionitis and/or neonatal sepsis. We systematically reviewed the literature and identified 200 relevant studies, five of which met the inclusion criteria. These studies reported on 381 patients, four studies reported on CRP as a predictor for histological chorioamnionitis, and four studies reported on CRP as a predictor for clinical chorioamnionitis. We were not able to identify a study in which 
CRP was used as a predictor for neonatal sepsis. CRP was moderately predictive for the identification of histological chorioamnionitis. Studies on clinical chorioamnionitis were too heterogenous to pool data. The single use of CRP to identify women and neonates at risk is not supported with current literature. However, as shown in Chapter 9, CRP has a predictive capacity in a prediction model to identify increased risk for neonatal sepsis.

Chapter 5 provides a detailed description of the study protocol. The PPROMEXIL trial was a multicentre randomised controlled open label trial, which was performed in 60 hospitals across The Netherlands. Women aged $>18$ years with preterm prelabour rupture of the fetal membranes between $34^{+0}$ and $37^{+0}$ weeks' gestation who had not delivered within 24 hours after rupture of membranes, were eligible for the trial.

If rupture of membranes occurred after $26^{+0}$ weeks and women had not delivered at $34^{+0}$ weeks they were also eligible for participation. Monochorionic multiple pregnancies, abnormal (non-reassuring) cardiotocogram, meconium stained amniotic fluid, signs of intrauterine infection, major fetal abnormalities, labour, HELLP syndrome or severe preeclampsia were exclusion criteria.

Women were randomised 1:1 for induction of labour ( $\mathrm{OL}$ ) or expectant management (EM). If women were randomised for induction of labour this was planned as soon as feasible but within 24 hours after randomisation, the same was done in women who were scheduled for a Caesarean Section (e.g. in case of a breech presentation). If a woman was assigned to expectant management, she was treated according to local protocol in either an inpatient or outpatient setting until $37^{+0}$ weeks, or whenever there was a need to terminate pregnancy on maternal or fetal indication.

Research nurses who counselled eligible women and collected data on all research sites staffed the trial. Alongside the PPROMEXIL trial an economic analysis (Chapter 10) and a Quality of Life questionnaire was performed (results not presented in this thesis) The primary outcome of the PPROMEXIL trial was the incidence of neonatal sepsis. Neonatal sepsis was defined as follows: (1) blood culture taken at birth found positive for bacteria or (2) two or more symptoms of infection within $72 \mathrm{~h}$ after birth, plus one of the following: (a) positive blood culture (culture proven sepsis); (b) C-reactive protein $>20$ mmol// (suspicion of sepsis); (c) positive surface cultures of a known virulent pathogen (suspicion of sepsis). When a local investigator classified a case as sepsis, or when criteria for sepsis were entered in the database, an independent panel of paediatricians judged the case to make a final diagnosis. Neonatal and maternal complications as well as mode of delivery and duration of hospital admission were registered as secondary outcome measurements.

A total of 520 women were needed to prove a reduction of neonatal sepsis of $7.5 \%$ when 
treated expectantly to $2.5 \%$ after induction of labour. We considered it clinically not plausible that loL would lead to an increased incidence and we therefore performed a power analysis on a one-sided test.

From January $1^{\text {st }} 2007$ until September $9^{\text {th }} 2009$ a total of 776 patients were eligible to participate in the PPROMEXIL trial. A total of 536 patients consented for participation and could be included in the trial. Four patients were excluded after randomisation. In Chapter 6 the results of the included 266 women (268 neonates) allocated to loL and 266 women (270 neonates) allocated to EM are presented. There was no difference in baseline characteristics. Mean maternal age at inclusion was 29.5 years, 56\% were nulliparous, $79 \%$ where of white ethnical origin and $24 \%$ smoked during pregnancy. EM prolonged pregnancy with 3.3 days. Neonatal sepsis occurred in seven (2.6\%) new-borns after loL and in 11 (4.1\%) new-borns when women were treated expectantly (relative risk [RR] $0.64,95 \%$ confidence interval [CI] 0.25 to 1.6). RDS was seen in 21 (7.8\%, loL) versus 17 neonates (6.3\%, EM) (RR 1.3; 95\% Cl 0.67 to 2.3). Hypoglycaemia (RR 2.2; $95 \% \mathrm{Cl} 1.4$ to 3.4) and hyperbilirubinemia (RR $1.5 ; 95 \% \mathrm{Cl} 1.1$ to 1.9 ) were seen significantly more often in the IoL group. Caesarean section was performed in 36 (13\%, IoL) versus 37 (14\%, EM) women (RR 0.98; $95 \% \mathrm{Cl} 0.64$ to 1.50). The risk for chorioamnionitis was reduced in the IoL group. No serious adverse events were reported.

Alongside the PPROMEXIL trial we updated a Cochrane meta-analysis on management of PPROM. Apart from the PPROMEXIL trial no additional trials could be included. Risk ratios were 1.06, 0.94, 0,87 and 0,83 for neonatal sepsis, culture proven neonatal sepsis, RDS and Caesarean Section rates, respectively. None of these risk ratios was statistically different.

Non-randomised women differed in baseline characteristics with respect to level of education (more educated), smoking (fewer smoked), maternal age (older), management (preferred EM) and gestation age at PPROM (earlier PPROM). Primary and secondary outcome were, however, comparable.

The incidence of neonatal sepsis was, however, lower than expected and therefore the PPROMEXIL study was underpowered. The liberal use of antibiotic therapy (overall $41 \%$ of all women received antibiotics during admission or labour) might have contributed to this lower incidence of neonatal sepsis. Although the PPROMEXIL study is the largest study published on this subject, the sample size was insufficient to demonstrate small differences on the primary outcome neonatal sepsis. Chorioamniontis was seen more often in the EM-group. Although relationships between chorioamnionitis and adverse neonatal outcome have been demonstrated in very premature neonates, we doubt whether these findings can be extrapolated to our population. In contrast, other secondary neonatal outcomes were not different between both treatment strategies or were seen more often 
in the IoL group (hypoglycaemia and hyperbilirubinemia). Taking into account that it has been shown that in preterm delivered infants, gestational age at delivery has a strong dose-dependent relationship with special educational need and that the updated metaanalysis did not favour loL, we conclude that in pregnancies complicated by PPROM between 34 and 37 weeks of gestation loL did not improve pregnancy outcome, compared to expectant management.

Because of the fact that the PPROMEXIL study was underpowered, and in view of uncertainty of the continuation of the other large on-going trial on the subject, the Australian PROMT trial, which was dependent on funding, we decided to set up a new trial called the PPROMEXIL-2 trial, aiming to randomise an additional 200 women. The results of this virtually similar trial as the PPROMEXIL trial are presented in Chapter 7.

Between December 2009 and January 2011241 women were eligible for the study. Fortythree women refused randomisation and after randomisation 3 women had to be excluded because it became apparent that they did not fulfil inclusion criteria. In total, we randomised 100 women to $\mathrm{IoL}$ and 95 to $\mathrm{EM}$.

Neonatal sepsis was seen in three (3.0\%) neonates in the loL-group versus 4 (4.1\%) neonates in the EM-group (RR $0.74,95 \% \mathrm{Cl} 0.17$ to 3.2). Women in the loL group delivered on average 3.5 days earlier. There were fewer Caesarean Sections in the loL group. This difference, although not significant, was partly because of a higher number of planned Caesarean Sections in the EM-group. Secondary outcomes were similar between the PPROMEXIL and the PPROMEXIL-2 trial in respect to the majority of maternal and neonatal outcomes. In contrast to the PPROMEXIL trial (Chapter 6) we found no difference in the incidence of hypoglycaemia and hyperbilirubinemia.

Including both PPROMEXIL trials in the existing Cochrane meta-analysis with respect to neonatal sepsis, RDS and Caesarean Section, no difference could be found between loL and EM based on more than 1400 neonates/women.

The incidence of neonatal sepsis in pregnancies complicated by near term PROM is low. Based on both PPROMEXIL trials and the updated meta-analysis we conclude that in our opinion there is enough evidence to prefer expectant management in these women.

Due to funding limitation the effect on adverse neonatal outcome such as stillbirth and neonatal death that have a (very) low incidence will probably never be found in a randomised controlled trial. We therefore performed a large retrospective study, using the Dutch nationwide perinatal database (PRN). Results from this study are presented in

\section{Chapter 8.}


Between 1999 and 2006 we included all singleton pregnancies complicated by PPROM between $34^{+0}$ and $37^{+0}$ weeks gestational age. The interval between rupture of membranes and birth had to be at least 24 hours. Spontaneous preterm deliveries between $34^{+0}$ and $37^{+0}$ weeks served as controls. Pregnancies with congenital anomalies, PE/HELLP syndrome and/or growth restriction were excluded.

The PRN combines delivery records (LVR) and neonatal records (LNR), but the latter is not mandatory and therefore coverage of the LNR is only $68 \%$. Because stillbirth occurs during pregnancy and is registered in the LVR record only, which covers $96 \%$ of all deliveries, we made a separate analysis for stillbirth.

In total 2,679 women within the PRN registry had PPROM between $34^{+0}$ and $37^{+0}$ weeks. 22,180 spontaneous deliveries without PPROM served as controls. Overall, 85 (3.2\%) neonates had a neonatal sepsis in the PPROM group versus $565(2.6 \%)$ in the control group (RR $1.25 ; 95 \% \mathrm{Cl} 0.99$ to 1.6$)$. The risk of meningitis was low $(0.3 \%)$ but significantly increased in the PPROM group ( $R R$ 3.7; $95 \% \mathrm{Cl} 1.6$ to 8.5). The risks of hypoglycaemia (RR 0.69), hyperbilirubinaemia (RR 0.77), oxygen therapy (RR 0.66), respiratory distress (RR 0.12 ) and airway infections (RR 0.42) were all significantly decreased in the PPROM group. Within the PROM group latency had no additional effect on the mode of delivery. In women with an interval between rupture of membranes between 72 and 119 hours and delivery, an increase in neonatal sepsis rate was seen compared to controls. Latency however decreases the incidences of hypoglycaemia and RDS. The cumulative stillbirth rate between $34^{+0}$ and $36^{+6}$ weeks in the PPROM group was significantly lower $(0,009 \%)$ than the stillbirth rate in controls $(0,066 \%)$ for the same period.

This retrospective database study has limitations with respect to coverage and accuracy; however, the size of this nationwide database minimizes this influence. Results of this study are in line with the PPROMEXIL trial. Although incidences of neonatal sepsis seem to be higher in the PROM group, other adverse neonatal outcome including stillbirth were significantly decreased when the interval between rupture of membranes and delivery increased. Therefore expectant management should be the treatment of choice in uncomplicated pregnancies with PROM near term.

Identification of women with a high risk for neonatal sepsis however might change expectant management into active management as neonatal sepsis is still a potentially life threatening risk. In Chapter $\mathbf{9}$ we describe the development of a prediction model for neonatal sepsis based on antepartum parameters. For the development of this prediction model we used data from both PPROMEXIL trials. Data from randomised as well as nonrandomised women who consented for follow-up registration were used for the model. In total data from 970 women could be analysed. In 33 neonates (3.4\%) a neonatal sepsis occurred. We evaluated 13 potential antepartum predictors for neonatal sepsis. Miss- 
ing data were imputed. We used multivariable logistic regression to develop a prediction model. Because this was the first model on this subject we allowed 5 events per predictor to be included in the model, dictating that a maximum of 6 predictors could be included in the model. A p-value of $<0.20$ was used to keep the predictor in the model. Bootstrapping internally validated the model. Discriminative ability of the model was expressed as the area under the receiver operating curve and a calibration was performed with a calibration plot as well as a Hosmer and Lemshow goodness-to-fit test. Overall performance of the model was quantified as the scaled Brier score.

The following antepartum characteristics were considered as candidate predictors: maternal age, parity, ethnicity, maternal smoking, maternal body mass index at start pregnancy, gestational age at PROM, antenatal administration of steroid, CRP level on admission, maternal with blood cell count, maternal temperature, positive vaginal culture (any pathogen and Group B streptococcus specific) and ante partum administration of antibiotics. From these antepartum parameters maternal age (OR 1.09 per year), maternal CRP (OR 1.01 per mmol/I), maternal temperature (OR 1.80 per oCelcius) and positive Group B streptococcus culture (OR 2.20) were associated with an increased risk of neonatal sepsis. The model had an area under the ROC-curve of 0.71 ( $95 \% \mathrm{Cl} 0.61$ to 0.82 ). The goodnessto-fit ( $p=0.32$ ) and adjusted Brier score $30 \%$ verified both good calibration and accuracy of the model.

We demonstrated that, based on ante partum parameters, it is possible to predict risk of neonatal sepsis more precisely.

In Chapter 10 we present the results of an economic analysis, which was performed alongside the PPROMEXIL trial. This economic analysis was done from a health care provider perspective, using a bottom-up approach to estimate resource utilisation, valued with unit costs reflecting actual costs. All direct medical costs were included. Costs were differentiated between antepartum cost, delivery costs and post partum costs and included maternal and neonatal admission, method of delivery, type of induction, outpatient visits, medication, laboratory testing, neonatal monitoring.

The mean costs per women were $€ 8,094$ for induction and $€ 7,340$ for expectant management (difference $€ 754 ; 95 \% \mathrm{Cl}-335$ to 1,802). The difference originates predominantly in the post partum period. Delivery costs were higher in women allocated to induction of labour. Antepartum costs were higher in expectant management. In conclusion, induction of labour seems not to reduce medical costs nor does it improve neonatal outcome.

This thesis has at least opened the global discussion about the management of late preterm prelabour rupture of membranes. With the completion of the Australian PROMT 
trial within the next year we will be able to execute an individual patient data (IPD) meta-analysis. This IPD meta-analysis will quite likely provide enough evidence to guide new international clinical guidelines.

Furthermore, the IPD meta-analysis will give the possibility to study subgroups and develop and validate prediction models in such way that we can individualise risk assessments for adverse neonatal (and maternal) outcome. 


\section{SAMENVATTING}

Ongeveer 3\% van alle zwangerschappen worden gecompliceerd door het prematuur breken van de vliezen voor het begin van de baring (PPROM). In 1.5\% van alle zwangerschappen gebeurt dit voor de $34^{\text {ste }}$ zwangerschapsweek en in $1.5 \%$ tussen de $34^{\text {ste }}$ en $37^{\text {ste }}$ zwangerschapsweek. Jaarlijks worden er in Nederland ongeveer 3.000 zwangerschappen gecompliceerd door PPROM. PPROM is geassocieerd met diverse complicaties zoals chorioamnionitis, opstijgende infecties, neonatale sepsis, prematuriteit, abruptio placentae, navelstreng prolaps en een mogelijk verhoogd risico op antepartum en intrapartum sterfte.

Internationale richtlijnen adviseren verschillend ten aanzien van de behandeling van patiënten met PPROM. Door gebrek aan voldoende goed wetenschappelijk onderzoek zijn adviezen gebaseerd op kleine series en zogenaamde expert opinions.

Het gebrek aan goed wetenschappelijk bewijs en een valide onderbouwing van de huidige adviezen rondom het beleid bij patiënten met PPROM was de aanleiding voor dit proefschrift en de Induction of Labour versus EXpectant management in women with Preterm Prelabour Rupture of Membranes between 34 and 37 weeks (PPROMEXIL) studie.

Bij aanvang van de PPROMEXIL studie hebben we een prior-to-belief vragenlijst afgenomen onder gynaecologen en arts-assistenten gynaecologie (Hoofdstuk 1). De kennis en verwachtingen onder de 236 (20\%) respondenten varieerde sterk. Tweeëntachtig procent van de respondenten gaf aan dat er voor het begin van de PPROMEXIL studie een afwachtend beleid werd gevoerd bij vrouwen met PPROM. Gevraagd naar het geschatte risico op een neonatale sepsis bij een afwachtend beleid was het antwoord gemiddeld $5 \%$, echter de ingeschatte risico's varieerden sterk (0\% tot 70\%). Een vergelijkbare brede spreiding tussen geschatte risico's werd gezien bij alle gevraagde uitkomsten. Deze bevinding is verontrustend, immers als het geschatte risico van een zorgverlener niet overeenkomt met het werkelijke risico dan reist de vraag in hoeverre het onderbuikgevoel van de zorgverlener (mede) bepalend is voor het voorgestelde beleid. Dit maakt implementatie studies naar de effectuering van studieresultaten in de dagelijkse praktijk noodzakelijk. Deze implementatie studies worden inmiddels verricht binnen het Dutch Consortium for Women's Healt and Reproductivity Studies (www.studies-obsgyn.nl), waarvan de PPROMEXIL studie één van de studies is.

De diagnose van gebroken vliezen kan bij de meerderheid (90\%) van de patiënten worden gemaakt op basis van anamnese en het feit dat er bij lichamelijk onderzoek duidelijk vochtverlies per vaginam is of dat dit wordt gezien bij speculum onderzoek. In Hoofdstuk 
2 hebben wij een systematische review verricht voor die groep vrouwen waarbij de diagnose gebroken vliezen onduidelijk blijft. Voor deze groep patiënten is een betrouwbare diagnostische test wenselijk. Met als doelpopulatie vrouwen met dubieus gebroken vliezen werd een systematische review uitgevoerd. Een brede zoekstrategie zonder taalrestricties leverde initieel meer dan 3800 titels van studies op. Na het screenen van titel en samenvatting op relevantie, bleven 146 studies over waarvan het volledige manuscript werd opgevraagd. In totaal werden 133 studies om verschillende redenen geëxcludeerd. Hierbij waren een onduidelijke beschrijving van de gebruikte test $(n=49)$ en een niet overeenkomende doelgroep (alleen patiënten met zeker gebroken vliezen of vruchtwater verkregen na een vruchtwaterpunctie; $n=33$ ) de meest voorkomende redenen.

De resterende 13 studies werden door een panel van experts gescoord. Uiteindelijk bleken drie studies te voldoen aan de vooraf gedefinieerde inclusie criteria. De gebruikte diagnostische test in de drie studies was verschillend $\mathrm{pH}$ meting, insulin-like growth factor binding protein-1 en alpha-fetoprotein). De verschillende methodes en de kleine studie omvang ( 8 tot 83 patiënten per studie) maakten het onmogelijk om een advies te kunnen geven over een diagnostische test bij vrouwen met dubieus gebroken vliezen.

Naar aanleiding van de resultaten in Hoofdstuk 2 werd op invitatie een aanvullend review geschreven waar de meest recente literatuur werd gereviewd. De resultaten van dit review staan beschreven in Hoofdstuk 3. Dezelfde zoekstrategie als gebruikt in Hoofdstuk 2 leverde zeven nieuwe studies op. Over het algemeen was het aantal patiënten per studie gering en gebruikten de onderzoekers verschillende "zilveren standaarden" als referentie test. De focus van de meeste studies was gericht op twee verschillende testen: insulinlike growth factor binding protein-1 (IGFBP-1) en placental $\alpha$-microglobulin-1 (PAMG-1). De accuratesse van IGFBP-1 en van PAMG-1 zijn in vergelijking met het verrichten van alleen een $\mathrm{pH}$-test of een varentest beter. Echter in vergelijking met de conventionele test methode (een combinatie van anamnese, varentest, $\mathrm{pH}$-test, speculumonderzoek en echografische onderzoek) zijn deze diagnostische testen niet beter. Ondanks het feit dat geen enkele studie de gouden standaard (amnioninfusie met een kleurstof) gebruikte en de geclaimde sensitiviteit en specificiteit van de testen met enige voorzichtigheid moeten worden geïnterpreteerd, blijkt dat het gebruik van deze nieuwe testen het vertrouwen van de behandelaar in het stellen van de juiste diagnose versterkt. In-vitro is PAMG-1 beter dan IGFBP-1 voor de detectie van vruchtwater wanneer vruchtwater wordt verdund met fysiologisch zout. Nieuwe biomarkers (zoals soluble intercellular adhesion molecule-1 [SICAM-1] en Axl receptor tyrosine kinase [AxI]) met een hoge concentratie in vruchtwater en een vrijwel afwezige concentratie in cervico-vaginale afscheiding lijken veelbelovend. Echter zo lang nieuwe diagnostische testen niet worden gevalideerd tegen 
de gouden standaard zal de betrouwbaarheid en toepasbaarheid van deze nieuwe testen altijd onderwerp van discussie blijven.

Bij de behandeling van vrouwen met PPROM zou een vroege opsporing van een intrauteriene infectie en van neonatale sepsis zeer welkom en behulpzaam zijn. In Hoofdstuk 4 hebben we geëvalueerd of C-reactive protein (CRP), een acuut fase eiwit, geschikt is om intra-uteriene infecties en/of neontale sepsis te kunnen voorspellen. We hebben de literatuur systematisch gereviewd, dit leverde 200 relevante studies op. Hiervan voldeden vijf aan de inclusie criteria. De studies includeerden gezamenlijk 381 patiënten. Vier studies evalueerden de toepasbaarheid van CRP als een voorspeller voor een histologische chorioamnionitis en vier studies evalueerden de toepasbaarheid van CRP als een voorspeller voor klinische chorioamnionitis. Geen enkele studie evalueerde de toepasbaarheid van CRP als een voorspeller voor neonatale sepsis. CRP bleek een matige voorspeller voor de identificatie van histologische chorioamnionitis te zijn. De studies die de toepasbaarheid van CRP als voorspeller van klinische chorioamnionitis onderzochten waren te heterogeen om de data te poolen. Het gebruik van alleen een CRP bepaling voor het identificeren van vrouwen met een verhoogd risico op het ontwikkelen van een chorioamnionitis of een neonatale sepsis kan op basis van de huidige literatuur niet worden ondersteund. Echter, zoals in Hoofdstuk $\mathbf{9}$ beschreven, is CRP wel een voorspellende parameter in het predictie model voor het identificeren van een verhoogd risico op neonatale sepsis.

Hoofdstuk 5 geeft een gedetailleerde beschrijving van het studieprotocol van de PPROMEXIL studie. De PPROMEXIL studie is een multicenter, gerandomiseerde, open label studie. De studie werd uitgevoerd in 60 Nederlandse ziekenhuizen. Vrouwen $>18$ jaar met gebroken vliezen gedurende ten minste 24 uur en met een zwangerschapsduur tussen de $34^{+0}$ en $37^{+0}$ weken konden worden geïncludeerd in de studie.

Indien de vliezen na $26^{+0}$ weken zwangerschapsduur waren gebroken en deze vrouwen bij $34^{+0}$ weken nog niet bevallen waren, konden zij eveneens deelnemen aan de studie. Monochoriale meerlingen, afwijkende cardiotocografie (CTG) registratie, meconium houdend vruchtwater, tekenen voor intra-uteriene infectie, ernstige congenitale afwijkingen, in partu zijn, HELLP syndroom of ernstige pre-eclampsie waren exclusie criteria.

Vrouwen werden gerandomiseerd 1:1 tussen inleiding van de baring ( $\mathrm{loL}$ ) en afwachtend beleid (EM). Indien een vrouw werd gerandomiseerd voor loL dan werd dit zo snel als mogelijk gepland na randomisatie. Begin van de inleiding van de baring moest plaatsvinden maximaal 24 uur na randomisatie. Hetzelfde gold voor vrouwen waarbij een sectio gepland was (b.v. bij een stuitligging). Indien een vrouw werd gerandomiseerd voor EM dan werd zij behandeld volgens lokaal protocol tot $37^{+0}$ weken zwangerschapsduur. Be- 
geleiding kon plaatsvinden door een klinische opname of door thuismonitoring. Indien er een maternale of foetale noodzaak was de zwangerschap voor de $37^{+0}$ weken te termineren dan werd de vrouw eerder ingeleid.

Counseling en dataverzameling van de studie vond lokaal plaats door researchmedewerkers. Parallel aan de PPROMEXIL studie werd een economische analyse (Hoofdstuk 10) en een kwaliteit-van-leven vragenlijst uitgevoerd (resultaten van de kwaliteit van leven studie zijn niet weergegeven in dit proefschrift).

De primaire uitkomst van de PPROMEXIL studie is de incidentie neonatale sepsis. Neonatale sepsis is gedefinieerd als: (1) een positieve bacteriële bloedkweek afgenomen bij geboorte, of (2) twee of meer symptomen van een infectie binnen 72 uur na geboorte plus een van de volgende items: (a) positieve bloedkweek (kweek positieve sepsis); (b) CRP > $20 \mathrm{mmol} /$ I (verdenking sepsis); (c) positieve oppervlakte kweek van een bekende virulente pathogeen (verdenking sepsis). Indien de lokale onderzoeker een neonaat classificeerde als sepsis of indien tenminste één van de criteria voor sepsis was ingevoerd in de database werd de casus beoordeeld door een onafhankelijk panel van kinderartsen voor een definitieve diagnose. Neonatale en maternale complicaties, manier van bevalling en duur van de ziekenhuisopname werden geregistreerd als secundaire uitkomsten. Om een afname van de incidentie sepsis van 7,5\% bij een afwachtend beleid naar 2,5\% bij een actief (inleidend) beleid aan te tonen waren 520 vrouwen nodig. Omdat wij het niet plausibel achtten dat het inleiden van de baring zou leiden tot een verhoogd risico op sepsis werd de power-analyse eenzijdig uitgevoerd.

Tussen 1 januari 2007 en 9 september 2009 werden 776 vrouwen gevraagd voor deelname aan de PPROMEXIL studie. In totaal gaven 536 vrouwen toestemming voor deelname en konden worden geïncludeerd in de PPROMEXIL studie. Na randomisatie moesten vier patiënten worden geëxludeerd. De resultaten van de 266 vrouwen (268 neonaten) die werden gerandomiseerd voor loL en de 266 vrouwen (270 neonaten) die werden gerandomiseerd voor EM staan beschreven in Hoofdstuk 6. Er was geen verschil in baseline karakteristieken. De gemiddelde maternale leeftijd bij inclusie bedroeg 29,5 jaar, 56\% van de vrouwen was nullipara, $79 \%$ hadden een blanke etniciteit en $24 \%$ rookten gedurende de zwangerschap. Een afwachtend beleid verlengde de zwangerschap met gemiddeld 3.3 dagen. Bij zeven kinderen (2.6\%) werd na het inleiden van de baring een neonatale sepsis gediagnostiseerd ten opzichte van elf kinderen (4.1\%) na afwachtend beleid (relatieve risico [RR] 0.64, 95\% betrouwbaarheidsinterval [CI] 0.25 tot 1.6$)$. Respiratory distress syndroom (RDS) werd gezien bij 21 (7.8\% loL-groep) versus 17 (6.3\%, EM-groep) neonaten (RR 1.3; 95\% Cl 0.67 tot 2.3). Hypoglykemie (RR 2.2; 95\% Cl 1.4 tot 3.4) en hyperbilirubinemie (RR 1.5; 95\% Cl 1.1 tot 1.9) werden significant vaker gezien bij neonaten na het 
inleiden van de baring. Zesendertig (13\%) vrouwen kregen een sectio in de loL-groep versus 37 (14\%) in de EM-groep (RR 0.98; 95\% Cl 0.64 tot 1.5). Het risico op chorioamnionitis was verhoogd in de EM-groep. Er deden zich gedurende de studie geen ernstige complicaties voor.

De resultaten van de PPROMEXIL studie werden opgenomen in een geüpdatete Cochrane meta-analyse. Behalve de PPROMEXIL studie waren er in de tussenliggende periode geen nieuwe studies. De risk ratio's van de geüpdatete meta-analyse waren 1.06, 0,94, 0,87 en 0,83 voor respectievelijk klinische neonatale sepsis, kweek bevestigde neonatale sepsis, RDS en het sectio percentage. Deze resultaten waren geen van allen statistisch verschillend.

De niet gerandomiseerde vrouwen die toestemming gaven voor het vervolgen van hun data verschilden van de gerandomiseerde vrouwen in relatie tot het opleidingsniveau (hoger opgeleid), roken (minder), maternale leeftijd (ouder), beleid (wens tot een afwachtend beleid) en zwangerschapsduur ten tijde van het breken van de vliezen (prematuurder). Primaire en secundaire uitkomsten van de niet gerandomiseerde vrouwen waren desalniettemin vergelijkbaar.

De incidentie neonatale sepsis was lager dan verwacht, derhalve was de PPROMEXIL studie onvoldoende gepowerd. Het laagdrempelig gebruik van antibiotica (41\% van de zwangere vrouwen kreeg antibiotica toegediend tijdens opname en/of de bevalling) kan hebben bijgedragen tot een lager dan verwachte incidentie neonatale sepsis. Ondanks het feit dat de PPROMEXIL studie de grootste gepubliceerde studie naar de behandeling van vrouwen met PPROM is, was de omvang van de onderzoeksgroep te klein om een (klein) verschil in de primaire uitkomst (neonatale sepsis) te kunnen aantonen. Wel werd chorioamnionitis vaker gezien wanneer een afwachtend beleid werd gevoerd. In de literatuur is er een relatie is aangetoond tussen chorioamnionitis en slechte neonatale uitkomsten. Deze relatie werd echter gevonden bij zeer premature neonaten en derhalve twijfelen wij of deze uitkomsten kunnen worden toegepast in onze populatie. In tegenstelling tot chorioamnionitis werden geen verschillen gevonden bij overige secundaire uitkomsten of was de incidentie van secundaire uitkomsten hoger (hypoglykemie en hyperbilirubinemie) bij de patiënten in de loL-groep. Dit samen met een eerder gevonden lineaire relatie tussen termijn van bevalling en noodzaak voor speciaal onderwijs (meer speciaal onderwijs bij kortere zwangerschapsduur bij bevalling) en de resultaten van de geüpdatete meta-analyse leidt tot de conclusie dat vrouwen waarbij de zwangerschap wordt gecompliceerd door PPROM tussen 34 en 37 weken het inleiden van de baring de uitkomst van de zwangerschap niet verbetert in vergelijking met een afwachtend beleid.

Door het gebrek aan power van de initiële PPROMEXIL studie en het mogelijk voortijdig 
stoppen van een vergelijkbare Australische studie door gebrek aan financiering, besloten we om een additionele tweede studie te starten onder de naam PPROMEXIL-2. In deze PPROMEXIL-2 studie werden 200 extra patiënten gerandomiseerd. De resultaten van de PPROMEXIL-2 studie waren overeenkomstig met die van de PPROMEXIL studie en worden beschreven in Hoofdstuk 7.

Tussen december 2009 en januari 2011 werden 241 vrouwen gevraagd voor deelname aan de studie. Drieënveertig gaven geen toestemming en drie vrouwen werden na randomisatie geëxcludeerd omdat ze retrospectief niet aan de inclusiecriteria voldeden. In totaal werden 100 vrouwen gerandomiseerd voor loL en 95 voor EM.

Neonatale sepsis werd gezien bij drie (3.0\%) neonaten in de loL-groep versus vier (4.1\%) in de EM-groep (RR $0.74,95 \% \mathrm{Cl} 0.17$ tot 3.2). Vrouwen in de loL-groep bevielen gemiddeld 3.5 dagen eerder. Het sectio percentage was lager in de loL-groep, echter dit verschil was niet statistisch significant en was grotendeels te verklaren door het hoge aantal geplande sectio's in de EM-groep. De secundaire uitkomsten van de PPROMEXIL-2 studie waren vergelijkbaar met die van de PPROMEXIL studie. In tegenstelling tot de PPROMEXIL studie (Hoofdstuk 6) vonden wij geen verschil in de incidentie hypoglykemie en hyperbilirubinemie.

Wanneer we de resultaten van de PPROMEXIL studies opnemen in de Cochrane metaanalyse kunnen we geen verschil vinden met betrekking tot neonatale sepsis, RDS en percentage sectio's in een totale groep van meer dan 1400 vrouwen/neonaten.

Samenvattend kan worden gesteld dat de incidentie van neonatale sepsis laag is. Gebaseerd op de resultaten van de PPROMEXIL studies en de geüpdatete Cochrane meta-analyse is onze conclusie dat er voldoende bewijs is om vrouwen met PPROM tussen de 34 en 37 weken zwangerschapsduur expectatief te behandelen.

Ernstige neonatale uitkomsten zoals intra-uteriene vruchtdood (IUVD) en neonatale sterfte hebben een (zeer) lage incidentie en zullen vrijwel nooit worden gevonden in randomised controlled trials. Om een uitspraak hierover te kunnen doen hebben wij een retrospectieve studie verricht gebruikmakend van de landelijke perinatale registratie (PRN). De resultaten van deze studie staan beschreven in Hoofdstuk 8.

Voor deze studie werden over de periode 1999 tot en met 2006 alle eenlingzwangerschappen met meer dan 24 uur gebroken vliezen en een zwangerschapsduur van $34^{+0}$ tot $37^{+0}$ weken geïncludeerd. Vrouwen die spontaan bevielen tussen $34^{+0}$ en $37^{+0}$ weken zonder langdurig gebroken vliezen dienden als controle patiënten. Exclusiecriteria waren congenitale afwijkingen, pre-eclampsie/HELLP syndroom en/of intra-uteriene groeivertraging.

De PRN combineert de bevallingsgegevens (LVR) met de neonatale registratie (LNR). Deze 
laatste is een niet verplichte registratie waardoor de LNR een dekking van slechts 68\% heeft. Omdat een IUVD in de LVR wordt geregistreerd, welke een registratiegraad van 95\% heeft, hebben we een separate analyse verricht voor deze uitkomst.

In totaal konden 2.679 vrouwen worden geïdentificeerd met PPROM tussen $34^{+0}$ en $37^{+0}$ weken. 22.180 vrouwen bevielen spontaan zonder PPROM en dienden als controle. De incidentie neonatale sepsis was 3.2\% (85 neonaten) in de PPROM groep versus 2.6\% (565 neonaten) in de controle groep (RR $1.2595 \% \mathrm{Cl} 0.99$ tot 1.6 ). Het risico van meningitis was laag $(0.3 \%)$ in de PPROM groep maar significant hoger dan in de controle groep (RR 3.7, $95 \% \mathrm{Cl} 1.6$ tot 8.5 ). De risico's van hypoglykemie (RR 0.69), hyperbilirubinemie (RR 0.77), noodzaak tot beademing (RR 0.66), RDS (RR 0.12) en luchtweginfecties (RR 0.42) waren allen significant verlaagd in de PPROM groep.

De duur van gebroken vliezen binnen de PPROM groep had geen effect op de manier van bevallen. In vergelijking met de controle groep had de groep vrouwen waarbij de vliezen tussen 72 en 119 uur gebroken waren een verhoogd risico op het krijgen van een neonatale sepsis. Toename van de duur van gebroken vliezen leidde echter ook tot een afname van het risico op hypoglykemie en RDS. De cumulatieve incidentie van een IUVD tussen $34^{+0}$ en $36^{+6}$ weken zwangerschapsduur was in de PPROM groep significant lager $(0.009 \%)$ dan in de controle groep $(0.066 \%)$.

Hoewel de retrospectieve opzet van deze studie haar beperkingen heeft met name in relatie tot de dekking en compleetheid van de data vermindert de grootte van de studie deze beperkingen. De resultaten van deze retrospectieve studie komen overeen met de resultaten van die in de PPROMEXIL studies. Hoewel de incidentie van neonatale sepsis in de PPROM groep hoger lijkt dan in de controle groep nemen de risico's op andere uitkomsten waaronder IUVD af naarmate het interval tussen het breken van de vliezen en de geboorte toeneemt. Ook deze studie ondersteunde het advies dat in verder ongecompliceerde zwangerschappen die worden gecompliceerd door PPROM tussen $34^{+0}$ en $37^{+0}$ weken zwangerschapsduur een afwachtend beleid de voorkeur heeft boven het inleiden van de baring.

De identificatie van vrouwen met een verhoogd risico op het krijgen van een kind met een neonatale sepsis kan het afwachtende beleid bij deze vrouwen veranderen in een actief beleid (inleiding van de baring) omdat een neonatale sepsis nog steeds een potentieel levensbedreigende aandoening is voor de neonaat. In Hoofdstuk $\mathbf{9}$ beschrijven we de ontwikkeling van een predictiemodel voor het identificeren van neonatale sepsis gebaseerd op antepartum parameters. Voor de ontwikkeling van dit predictiemodel hebben we gebruik gemaakt van de data uit de PPROMEXIL studies. Zowel de data van de gerandomiseerde als de niet gerandomiseerde patiënten werden hiervoor gebruikt. In totaal 
konden zodoende de data van 970 vrouwen worden geanalyseerd. Bij 33 (3.4\%) van de neonaten werd een neonatale sepsis gediagnostiseerd. Dertien potentieel voorspellende antepartum parameters werden geëvalueerd in dit model. Omdat dit het eerste predictiemodel was voor het identificeren van neonatale sepsis bij vrouwen met PPROM werden 5 events per predictor geaccepteerd. Derhalve kon een maximum van 6 parameters worden opgenomen in het predictiemodel. Om een parameter in het model te laten werd een afkapwaarde van $p<0.20$ gekozen. De validatie van het model werd gedaan door middel van bootstrapping. Het discriminatieve vermogen van het model werd uitgedrukt als de area under the receiver operating curve en het model werd gekalibreerd met een kalibratie-plot en een Hosmer en Lemshow goodness-to-fit test. De bruikbaarheid van het model werd gekwantificeerd door middel van een scaled Brier score.

Maternale leeftijd, pariteit, etniciteit, roken, maternale body mass index bij het begin van de zwangerschap, zwangerschapsduur ten tijde van het breken van de vliezen, toediening van corticosteroïden, CRP waarde bij opname, leukocyten bepaling, maternale temperatuur, positieve vaginakweek (ieder pathogeen en groep B streptococcus specifiek) en toediening van antibiotica voor de baring werden geanalyseerd als mogelijk voorspellende parameters. Hiervan bleken maternale leeftijd (OR 1.09 per jaar), maternaal CRP waarde (OR 1.01 per mmol/I), maternale temperatuur (OR 1.80 per ${ }^{\circ} \mathrm{Celcius}$ ) en positieve groep B streptococcus kweek (OR 2.20) geassocieerd met een verhoogd risico op het krijgen van een kind met een neonatale infectie. Het model had een area under the ROC-curve van 0.71 (95\% Cl 0.61 tot 0.82 ). De goodness-to-fit ( $p=0.32$ ) en adjusted Brier score (30\%) toonden een goede kalibratie en accuratesse van het model.

Concluderend is het dus mogelijk om neonatale sepsis op basis van antepartum parameters beter te kunnen voorspellen. Echter een betere voorspelling dan een maximaal risico van $20 \%$ is niet te maken.

In Hoofdstuk 10 worden de resultaten van de economische analyse gepresenteerd welke gelijktijdig met de PPROMEXIL studie werd uitgevoerd. Deze economische analyse is verricht vanuit het oogpunt van de behandelaar. Hierbij is gebruikt gemaakt van een zogenaamde bottom-up benadering om een schatting te maken van zorgkosten. Alle directe medische kosten werden meegenomen. De kosten werden onderverdeeld in antepartum kosten, durante partum kosten en post partum kosten. De kosten bevatten maternale en neonatale opname kosten, kosten voor de bevalling, methode van inleiding, polikliniekbezoeken, medicatie, laboratorium onderzoeken en neonatale monitoringskosten.

De gemiddelde kosten per vrouw waren $€ 8.094$ voor patiënten in de loL-groep en $€ 7.340$ voor patiënten in de EM-groep (verschil $€ 754,95 \% \mathrm{Cl} €-355$ tot $€ 1.802$ ). Het verschil wordt voornamelijk veroorzaakt door de post partum kosten. Kosten rondom de 
bevalling waren hoger in de loL-groep. Antepartum kosten waren hoger in de EM-groep. Concluderend kan worden gesteld dat inleiden van de baring de zorgkosten niet verminderd noch verbetert het de neonatale uitkomsten.

Dit proefschrift heeft de wereldwijde discussie over de behandeling van patiënten met PPROM heropend. Zodra de inclusie van de nog lopende Australische PROMT studie gereed is, zullen we in staat zijn een individuele patiënten data (IPD) meta-analyse uit te voeren. Deze IPD meta-analyse zal zeer waarschijnlijk voldoende wetenschappelijk bewijs leveren om internationale richtlijnen verder aan te passen.

Daarnaast geeft de IPD meta-analyse ons de mogelijkheid om subgroepen beter te anaIyseren en predictiemodellen verder extern te valideren zodat we een verdere stap kunnen maken in het individualiseren van het risico op (ernstige) neonatale en maternale uitkomsten. 



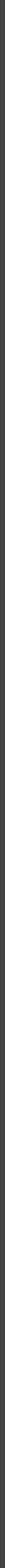




\section{APPENDIX LIST OF CO-AUTHORS AND COLLABORATORS ACKNOWLEDGEMENT REFERENCE LIST PUBLICATIONS \& PRESENTATIONS}




\begin{tabular}{|c|c|c|c|c|}
\hline & $\begin{array}{l}\text { PPROM } \\
\text { (>24h) }\end{array}$ & Control & $\begin{array}{l}\text { Relative risk } \\
(95 \% \mathrm{Cl})\end{array}$ & p-value \\
\hline Patients (n) & 374 & 3971 & & \\
\hline \multicolumn{5}{|l|}{ INFECTIONS } \\
\hline Neonatal Sepsis, $\mathrm{n}(\%)$ & $19(5.1 \%)$ & $240(6.0 \%)$ & 0.84 (0.53 to 1.33$)$ & 0.452 \\
\hline Meningitis, $n$ (\%) & $2(0.5 \%)$ & $4(0.1 \%)$ & 5.3 (0.98 to 28.9 ) & 0.031 \\
\hline \multicolumn{5}{|c|}{ METABOLIC/GASTROINTESTINAL MORBIDITY } \\
\hline Hypoglycemia, n (\%) ${ }^{\text {a }}$ & $27(7.2 \%)$ & $338(8.5 \%)$ & 0.85 (0.58 to 1.24$)$ & 0.389 \\
\hline Hyperbilirubinemia, n (\%) & $96(26 \%)$ & $1192(30 \%)$ & 0.86 (0.72 to 1.02$)$ & 0.078 \\
\hline Necrotizing enterocolitis, $\mathrm{n}(\%)$ & $0(0 \%)$ & $6(0.2 \%)$ & $\mathrm{n} / \mathrm{a}$ & 0.452 \\
\hline \multicolumn{5}{|l|}{ RESPIRATORY MORBIDITY } \\
\hline $\begin{array}{l}\text { Oxygen therapy } \geq 24 \text { hours, } \\
n(\%)\end{array}$ & $40(11 \%)$ & $574(14 \%)$ & $\begin{array}{lrl}0.74 & (0.55 & \text { to } \\
1.001) & \end{array}$ & 0.046 \\
\hline $\begin{array}{l}\text { Respiratory Distress Syndrome, } \\
\mathrm{n}(\%)\end{array}$ & $11(2.9 \%)$ & $238(6.0 \%)$ & 0.49 (0.27 to 0.89$)$ & 0.015 \\
\hline $\begin{array}{l}\text { Respiratory problems (no RDS), } \\
n(\%)\end{array}$ & $28(7.5 \%)$ & $490(12 \%)$ & 0.61 (0.42 to 0.87 ) & 0.006 \\
\hline $\begin{array}{l}\text { Bronchopulmonary Disease, } \\
\mathrm{n}(\%)\end{array}$ & $0(0 \%)$ & $0(0 \%)$ & $\mathrm{n} / \mathrm{a}$ & $\mathrm{n} / \mathrm{a}$ \\
\hline Airway infections, n (\%) & $0(0 \%)$ & $35(0.9 \%)$ & $\mathrm{n} / \mathrm{a}$ & 0.068 \\
\hline \multicolumn{5}{|l|}{ NEUROLOGIC MORBIDITY } \\
\hline Intracranial Hemorrhage, n (\%) & $1(0.3 \%)$ & $17(0.4 \%)$ & 0.62 (0.08 to 4.68$)$ & 0.644 \\
\hline $\begin{array}{l}\text { Hypoxic Ischemic Encephalopa- } \\
\text { thy, n (\%) }\end{array}$ & $1(0.3 \%)$ & $13(0.3 \%)$ & 0.82 (0.11 to 6.23 ) & 0.845 \\
\hline $\begin{array}{l}\text { Cerebral Ischemia (not PVL), } \\
\mathrm{n}(\%)\end{array}$ & $1(0.3 \%)$ & $7(0.2 \%)$ & 1.52 (0.19 to 12.3 ) & 0.694 \\
\hline $\begin{array}{l}\text { Periventricular Leucomalacia, } \\
\mathrm{n}(\%)\end{array}$ & $0(0 \%)$ & $8(0.2 \%)$ & $\mathrm{n} / \mathrm{a}$ & 0.385 \\
\hline Convulsions, n (\%) & $1(0.3 \%)$ & $12(0.3 \%)$ & 0.88 (0.11 to 6.79$)$ & 0.906 \\
\hline Neonatal death $(n, \%)$ & $1(0.3 \%)$ & $11(0.3 \%)$ & 0.97 (0.13 to 7.46$)$ & 0.973 \\
\hline \multicolumn{5}{|l|}{ MODE OF DELIVERY } \\
\hline Spontaneously, n (\%) & $286(76 \%)$ & $3180(80 \%)$ & 0.96 (0.90 to 1.01$)$ & 0.097 \\
\hline $\begin{array}{l}\text { Vaginal instrumental delivery, } \\
\mathrm{n}(\%)\end{array}$ & $45(12 \%)$ & $326(8.2 \%)$ & 1.47 (1.09 to 1.97$)$ & 0.011 \\
\hline Cesarean Section, n (\%) & $43(10 \%)$ & $465(12 \%)$ & $0.98(0.73$ to 1.32$)$ & 0.903 \\
\hline
\end{tabular}

(PP)ROM - (Premature Prelabor) Rupture of membranes; PVL - Periventricular Leucomalacia

a Hypoglycaemia in neonates of non-diabetic mothers

Table A.1 | Relative risk for neonatal morbidity and mode of delivery gestational age at rupture of membrane subgroup $34^{+0}$ to $34^{+6}$ weeks 


\begin{tabular}{|c|c|c|c|c|}
\hline & $\begin{array}{l}\text { PPROM } \\
\text { (>24h) }\end{array}$ & Control & $\begin{array}{l}\text { Relative risk } \\
(95 \% \mathrm{Cl})\end{array}$ & p-value \\
\hline Patients (n) & 784 & 6659 & & \\
\hline \multicolumn{5}{|l|}{ INFECTIONS } \\
\hline Neonatal Sepsis, $n(\%)$ & $34(4.3 \%)$ & $191(2.9 \%)$ & 1.51 (1.06 to 2.16$)$ & 0.023 \\
\hline Meningitis, $n$ (\%) & $3(0.4 \%)$ & $5(0.08 \%)$ & $5.10(1.22$ to 21.3$)$ & 0.013 \\
\hline \multicolumn{5}{|c|}{ METABOLIC/GASTROINTESTINAL MORBIDITY } \\
\hline Hypoglycemia, n (\%) a & $62(7.9 \%)$ & $651(9.8 \%)$ & 0.81 (0.63 to 1.04$)$ & 0.093 \\
\hline Hyperbilirubinemia, n (\%) & $152(19 \%)$ & $1524(23 \%)$ & $0.85(0.73$ to 0.98$)$ & 0.027 \\
\hline Necrotizing enterocolitis, $\mathrm{n}(\%)$ & $0(0 \%)$ & $6(0.09 \%)$ & $\mathrm{n} / \mathrm{a}$ & 0.400 \\
\hline \multicolumn{5}{|l|}{ RESPIRATORY MORBIDITY } \\
\hline $\begin{array}{l}\text { Oxygen therapy } \geq 24 \text { hours, } \\
n(\%)\end{array}$ & $44(5.6 \%)$ & $539(8.1 \%)$ & 0.69 (0.51 to 0.93 ) & 0.014 \\
\hline $\begin{array}{l}\text { Respiratory Distress Syndrome, } \\
\mathrm{n}(\%)\end{array}$ & $7(0.9 \%)$ & $149(2.2 \%)$ & 0.40 (0.19 to 0.85$)$ & 0.013 \\
\hline $\begin{array}{l}\text { Respiratory problems (no RDS), } \\
\mathrm{n}(\%)\end{array}$ & $42(5.4 \%)$ & $510(7.7 \%)$ & 0.70 (0.51 to 0.95$)$ & 0.020 \\
\hline $\begin{array}{l}\text { Bronchopulmonary Disease, } \\
\mathrm{n}(\%)\end{array}$ & $0(0 \%)$ & $0(0 \%)$ & $\mathrm{n} / \mathrm{a}$ & $\mathrm{n} / \mathrm{a}$ \\
\hline Airway infections, n (\%) & $4(0.5 \%)$ & $54(0.8 \%)$ & 0.63 (0.23 to 1.73 ) & 0.365 \\
\hline \multicolumn{5}{|l|}{ NEUROLOGIC MORBIDITY } \\
\hline Intracranial Hemorrhage, $\mathrm{n}(\%)$ & $0(0 \%)$ & $9(0.1 \%)$ & $\mathrm{n} / \mathrm{a}$ & 0.303 \\
\hline $\begin{array}{l}\text { Hypoxic Ischemic Encephalopa- } \\
\text { thy, n (\%) }\end{array}$ & $1(0.1 \%)$ & $13(0.2 \%)$ & 0.65 (0.09 to 4.99 ) & 0.679 \\
\hline $\begin{array}{l}\text { Cerebral Ischemia (not PVL), } \\
\mathrm{n}(\%)\end{array}$ & $1(0.1 \%)$ & $3(0.05 \%)$ & 2.83 (0.29 to 27.2 ) & 0.346 \\
\hline $\begin{array}{l}\text { Periventricular Leucomalacia, } \\
\mathrm{n}(\%)\end{array}$ & $0(0 \%)$ & $3(0.05 \%)$ & $\mathrm{n} / \mathrm{a}$ & 0.552 \\
\hline Convulsions, n (\%) & $2(0.3 \%)$ & $16(0.3 \%)$ & $1.06(0.24$ to 4.61$)$ & 0.936 \\
\hline Neonatal death $(n, \%)$ & $0(0 \%)$ & $12(0.2 \%)$ & $\mathrm{n} / \mathrm{a}$ & 0.234 \\
\hline \multicolumn{5}{|l|}{ MODE OF DELIVERY } \\
\hline Spontaneously, n (\%) & $626(80 \%)$ & $5463(82 \%)$ & 0.97 (0.94 to 1.01$)$ & 0.132 \\
\hline $\begin{array}{l}\text { Vaginal instrumental delivery, } \\
\mathrm{n}(\%)\end{array}$ & $100(13 \%)$ & $569(8.5 \%)$ & 1.49 (1.22 to 1.82$)$ & $<0.0001$ \\
\hline Cesarean Section, n (\%) & $58(7.4 \%)$ & $627(9.4 \%)$ & $0.79(0.61$ to 1.02$)$ & 0.065 \\
\hline
\end{tabular}

(PP)ROM - (Premature Prelabor) Rupture of membranes; PVL - Periventricular Leucomalacia

${ }^{a}$ Hypoglycaemia in neonates of non-diabetic mothers

Table A.2 | Relative risk for neonatal morbidity and mode of delivery gestational age at rupture of membrane subgroup $35^{+0}$ to $35^{+6}$ weeks 


\begin{tabular}{|c|c|c|c|c|}
\hline & $\begin{array}{l}\text { PPROM } \\
\text { (>24h) }\end{array}$ & Control & $\begin{array}{l}\text { Relative risk } \\
\text { (95\%Cl) }\end{array}$ & p-value \\
\hline Patients ( $\mathrm{n}$ ) & 1512 & 11550 & & \\
\hline \multicolumn{5}{|l|}{ INFECTIONS } \\
\hline Neonatal Sepsis, n (\%) & $32(2.1 \%)$ & $134(1.2 \%)$ & 1.82 (1.25 to 2.67$)$ & 0.002 \\
\hline Meningitis, $\mathrm{n}(\%)$ & $3(0.2 \%)$ & $9(0.08 \%)$ & 2.56 (0.69 to 9.46$)$ & 0.146 \\
\hline \multicolumn{5}{|c|}{ METABOLIC/GASTROINTESTINAL MORBIDITY } \\
\hline Hypoglycemia, n (\%) a & $76(5.0 \%)$ & $986(8.5 \%)$ & 0.59 (0.47 to 0.74$)$ & $<0.001$ \\
\hline Hyperbilirubinemia, n (\%) & $93(6.2 \%)$ & $957(8.3 \%)$ & 0.74 (0.60 to 0.91$)$ & 0.004 \\
\hline Necrotizing enterocolitis, $n(\%)$ & $0(0 \%)$ & $2(0.02 \%)$ & $\mathrm{n} / \mathrm{a}$ & 0.609 \\
\hline \multicolumn{5}{|l|}{ RESPIRATORY MORBIDITY } \\
\hline $\begin{array}{l}\text { Oxygen therapy } \geq 24 \text { hours, } \\
\text { n (\%) }\end{array}$ & $33(2.2 \%)$ & $360(3.1 \%)$ & $\begin{array}{l}0.70(0.49 \text { to } \\
0.996)\end{array}$ & 0.046 \\
\hline $\begin{array}{l}\text { Respiratory Distress Syndrome, } \\
\mathrm{n}(\%)\end{array}$ & $2(0.1 \%)$ & $69(0.6 \%)$ & 0.22 (0.05 to 0.90$)$ & 0.021 \\
\hline $\begin{array}{l}\text { Respiratory problems (no RDS), } \\
\mathrm{n}(\%)\end{array}$ & $41(2.7 \%)$ & $436(3.8 \%)$ & 0.72 (0.52 to 0.98$)$ & 0.038 \\
\hline $\begin{array}{l}\text { Bronchopulmonary Disease, } \\
\text { n (\%) }\end{array}$ & $0(0 \%)$ & $0(0 \%)$ & $\mathrm{n} / \mathrm{a}$ & $\mathrm{n} / \mathrm{a}$ \\
\hline Airway infections, n (\%) & $3(0.2 \%)$ & $48(0.4 \%)$ & 0.48 (0.15 to 1.53$)$ & 0.203 \\
\hline \multicolumn{5}{|l|}{ NEUROLOGIC MORBIDITY } \\
\hline Intracranial Hemorrhage, $\mathrm{n}(\%)$ & $0(0 \%)$ & $8(0.07 \%)$ & $n / a$ & 0.306 \\
\hline $\begin{array}{l}\text { Hypoxic Ischemic Encephalopa- } \\
\text { thy, } \mathrm{n}(\%)\end{array}$ & $2(0.1 \%)$ & $14(0.1 \%)$ & 1.09 (0.25 to 4.80$)$ & 0.908 \\
\hline $\begin{array}{l}\text { Cerebral Ischemia (not PVL), } \\
\mathrm{n}(\%)\end{array}$ & $2(0.1 \%)$ & $4(0.03 \%)$ & $3.8(0.70$ to 20.9$)$ & 0.096 \\
\hline $\begin{array}{l}\text { Periventricular Leucomalacia, } \\
\mathrm{n}(\%)\end{array}$ & $0(0 \%)$ & $2(0.02 \%)$ & $\mathrm{n} / \mathrm{a}$ & 0.601 \\
\hline Convulsions, n (\%) & $4(0.3 \%)$ & $14(0.1 \%)$ & 2.18 (0.72 to 6.62$)$ & 0.158 \\
\hline Neonatal death $(n, \%)$ & $0(0 \%)$ & $14(0.1 \%)$ & $n / a$ & 0.176 \\
\hline \multicolumn{5}{|l|}{ MODE OF DELIVERY } \\
\hline Spontaneously, n (\%) & $\begin{array}{l}1158 \\
(77 \%)\end{array}$ & $\begin{array}{l}9304 \\
(81 \%)\end{array}$ & 0.95 (0.92 to 0.98$)$ & $<0.0001$ \\
\hline $\begin{array}{l}\text { Vaginal instrumental delivery, } \\
\mathrm{n}(\%)\end{array}$ & $\begin{array}{c}250 \\
(17 \%)\end{array}$ & $\begin{array}{c}1121 \\
(9.7 \%)\end{array}$ & 1.70 (1.50 to 1.93$)$ & $<0.0001$ \\
\hline Cesarean Section, n (\%) & $\begin{array}{c}104 \\
(7.0 \%)\end{array}$ & $\begin{array}{c}1125 \\
(9.7 \%)\end{array}$ & 0.71 (0.58 to 0.86$)$ & $<0.0001$ \\
\hline
\end{tabular}

(PP)ROM - (Premature Prelabor) Rupture of membranes; PVL - Periventricular Leucomalacia

${ }^{\text {a }}$ Hypoglycaemia in neonates of non-diabetic mothers

Table A.3 | Relative risk for neonatal morbidity and mode of delivery gestational age at rupture of membrane subgroup $36^{+0}$ to $36^{+6}$ weeks 


\begin{tabular}{|c|c|c|c|c|}
\hline & No Sepsis & $\begin{array}{l}\text { Neonatal } \\
\text { Sepsis }\end{array}$ & OR $(95 \% \mathrm{Cl})^{\mathrm{a}}$ & OR $(95 \% \mathrm{Cl})^{b}$ \\
\hline Control (<24h of ROM, n,\%) & $3,731(94 \%)$ & $240(6.0 \%)$ & 1.0 & - \\
\hline \multicolumn{5}{|l|}{ STUDY POPULATION } \\
\hline ROM interval 24-30h $(n, \%)$ & $66(97 \%)$ & $2(2.9 \%)$ & $\begin{array}{c}0.471 \\
\text { (0.12 to } 1.96)\end{array}$ & 1.0 \\
\hline ROM interval 31-72h (n,\%) & $118(92 \%)$ & $10(7.8 \%)$ & $\begin{array}{c}1.317 \\
\text { (0.68 to } 2.55)\end{array}$ & $\begin{array}{c}2.797 \\
(0.60 \text { to } 13.2)\end{array}$ \\
\hline ROM interval $>72 h(n, \%)$ & $171(96 \%)$ & 7 (3.9\%) & $\begin{array}{c}0.636 \\
(0.30 \text { to } 1.37)\end{array}$ & $\begin{array}{c}1.351 \\
\text { (0.27 to } 6.67)\end{array}$ \\
\hline
\end{tabular}

a Odd ratio's are given between control and interval subgroups of the study population

b Odd ratio's are given between a rupture of membrane interval between 24 and 30 hours and the other interval groups of the study population

Abbreviations: $\mathrm{Cl}$ - Confidence interval; OR - Odds ratio; (PP)ROM - (Premature prelabor) rupture of membranes

Table A.5 | Neonatal sepsis related to interval between rupture of membranes and delivery (34 weeks)

\begin{tabular}{|c|c|c|c|c|}
\hline & No Sepsis & $\begin{array}{l}\text { Neonatal } \\
\text { Sepsis }\end{array}$ & OR $(95 \% \mathrm{Cl})^{a}$ & OR $(95 \% \mathrm{Cl})^{b}$ \\
\hline Control ( $<24 \mathrm{~h}$ of ROM, n,\%) & $6,468(97 \%)$ & $191(2.9 \%)$ & 1.0 & - \\
\hline \multicolumn{5}{|l|}{ STUDY POPULATION } \\
\hline ROM interval 24-30h $(n, \%)$ & $136(96 \%)$ & $6(4.2 \%)$ & $\begin{array}{c}1.494 \\
\text { (0.65 to } 3.44)\end{array}$ & 1.0 \\
\hline ROM interval 31-72h $(n, \%)$ & $319(96 \%)$ & $15(4.5 \%)$ & $\begin{array}{c}1.592 \\
\text { (0.93 to } 2.73)\end{array}$ & $\begin{array}{c}1.066 \\
\text { (0.41 to } 2.81)\end{array}$ \\
\hline ROM interval $>72 h(n, \%)$ & 295 (96\%) & $13(4.2 \%)$ & $\begin{array}{c}1.492 \\
\text { (0.84 to } 2.65)\end{array}$ & $\begin{array}{c}0.999 \\
\text { (0.37 to } 2.68)\end{array}$ \\
\hline
\end{tabular}

a Odd ratio's are given between control and interval subgroups of the study population

b Odd ratio's are given between a rupture of membrane interval between 24 and 30 hours and the other interval groups of the study population

Abbreviations: $\mathrm{Cl}$ - Confidence interval; OR - Odds ratio; (PP)ROM - (Premature prelabor) rupture of membranes

Table A.6 | Neonatal sepsis related to interval between rupture of membranes and delivery (35 weeks) 


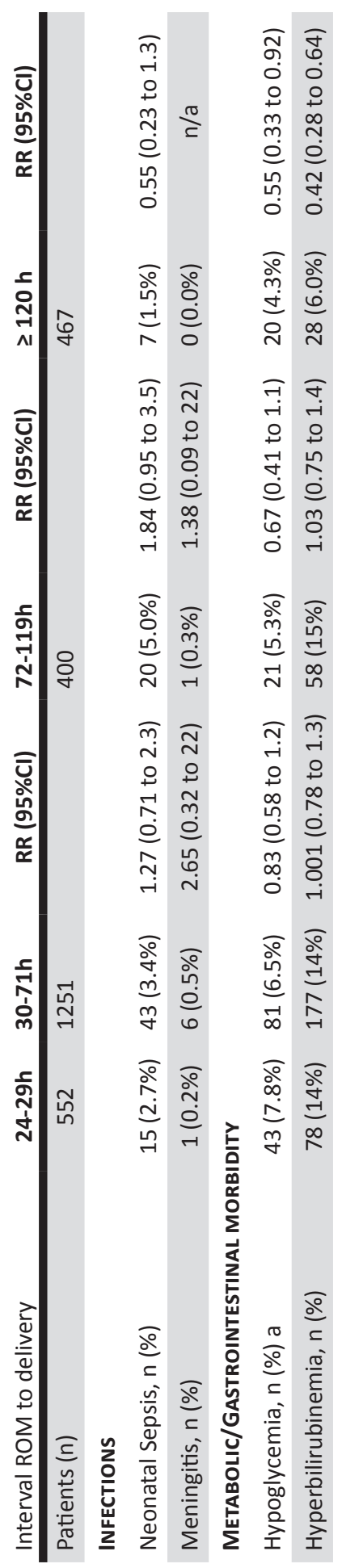




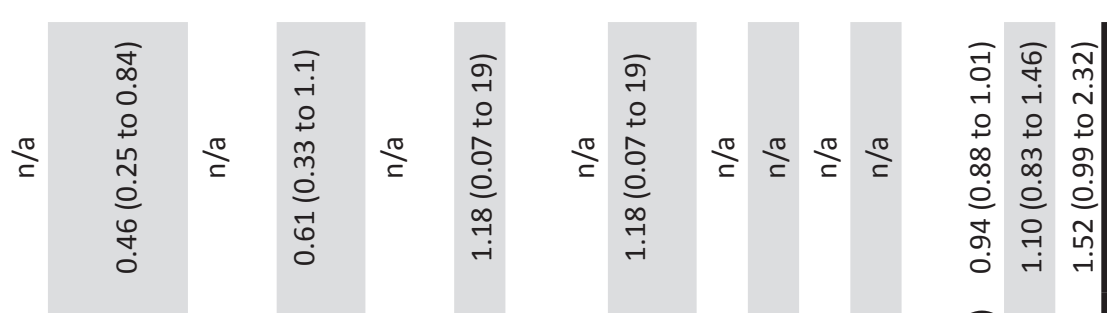

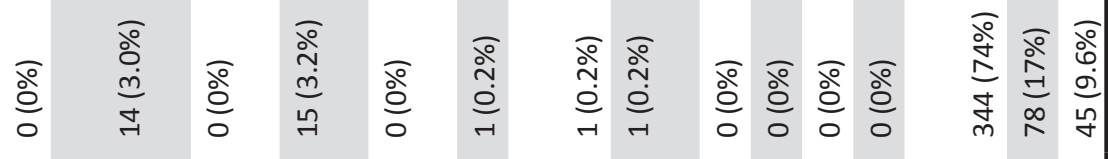

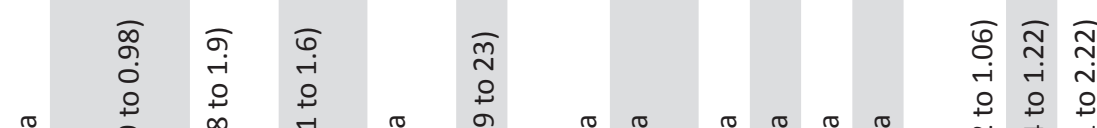

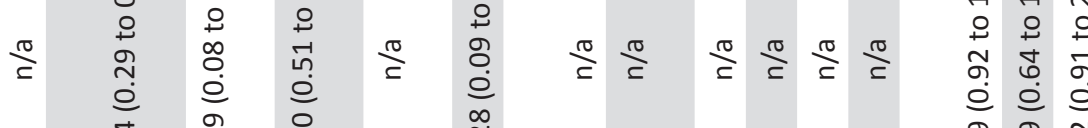

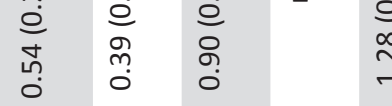

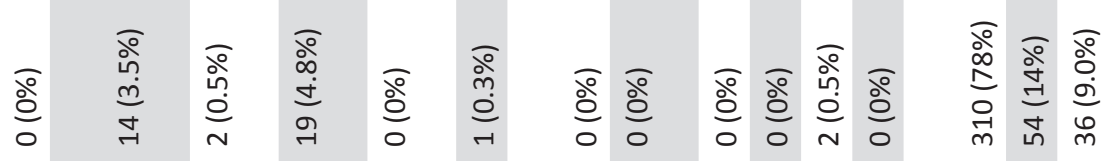

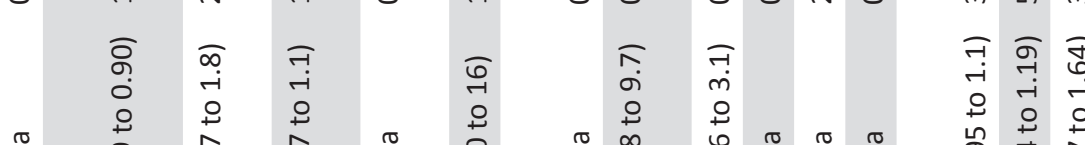

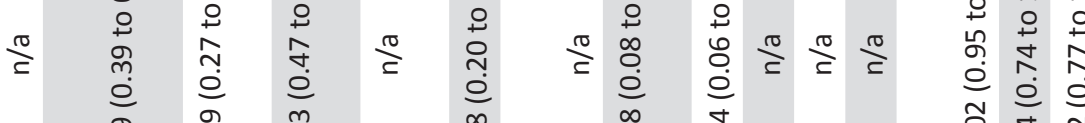

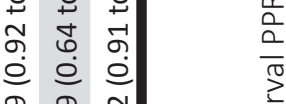

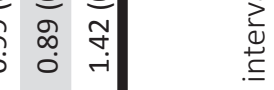

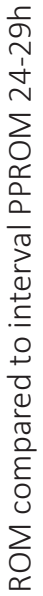

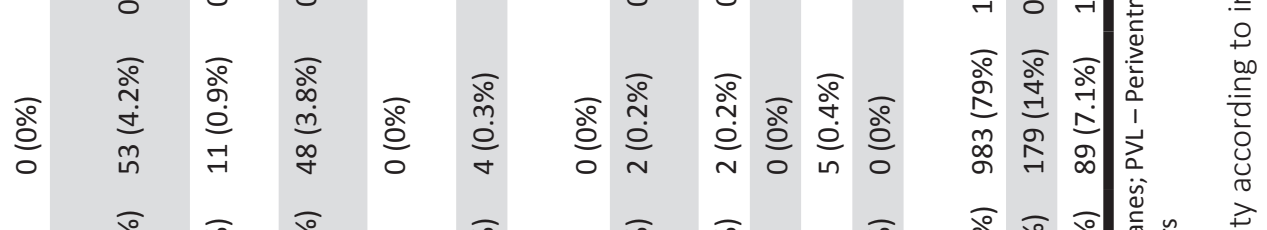

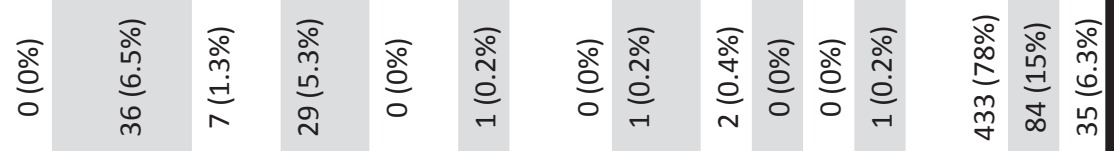

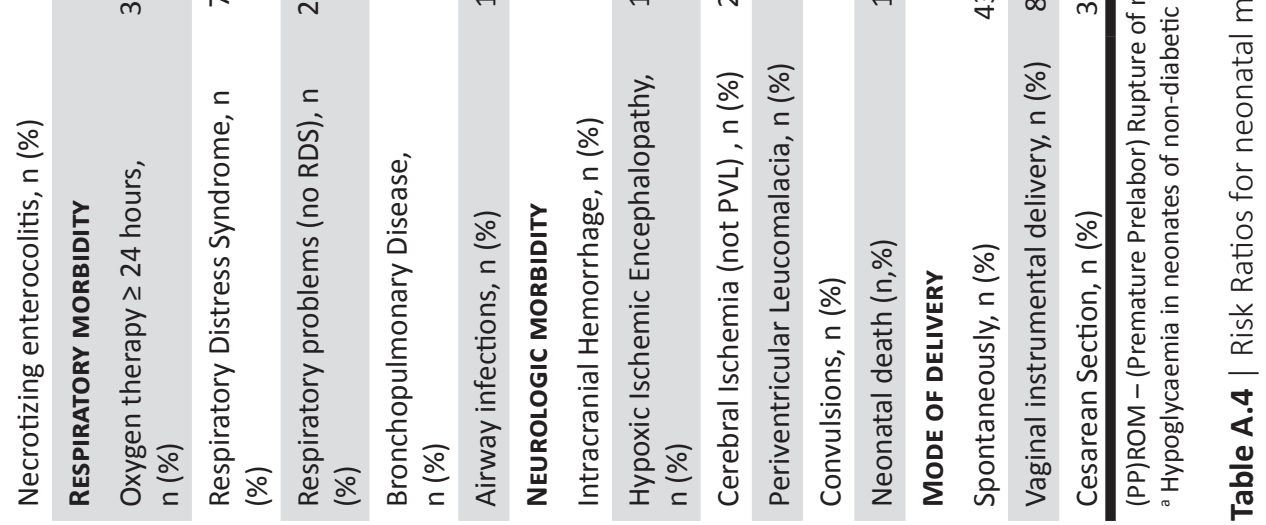




\begin{tabular}{|c|c|c|c|c|}
\hline & No Sepsis & $\begin{array}{c}\text { Neonatal } \\
\text { Sepsis }\end{array}$ & OR $(95 \% \mathrm{Cl})^{a}$ & OR $(95 \% \mathrm{Cl})^{b}$ \\
\hline Control (<24h of ROM, n,\%) & 11,416 (99\%) & $134(1.1 \%)$ & 1.0 & - \\
\hline \multicolumn{5}{|l|}{ STUDY POPULATION } \\
\hline ROM interval 24-30h $(n, \%)$ & 335 (98\%) & $7(2.1 \%)$ & $\begin{array}{c}1.780 \\
\text { (0.83 to } 3.84)\end{array}$ & 1.0 \\
\hline ROM interval 31-72h $(n, \%)$ & 771 (98\%) & $18(2.3 \%)$ & $\begin{array}{c}1.989 \\
(1.21 \text { to } 3.27)\end{array}$ & $\begin{array}{c}1.117 \\
\text { (0.46 to } 2.70)\end{array}$ \\
\hline ROM interval $>72 h(n, \%)$ & 374 (98\%) & $7(1.8 \%)$ & $\begin{array}{c}1.595 \\
\text { (0.74 to } 3.43)\end{array}$ & $\begin{array}{c}0.896 \\
\text { (0.31 to } 2.58)\end{array}$ \\
\hline
\end{tabular}

a Odd ratio's are given between control and interval subgroups of the study population

' Odd ratio's are given between a rupture of membrane interval between 24 and 30 hours and the other interval groups of the study population

Abbreviations: $\mathrm{Cl}$ - Confidence interval; OR - Odds ratio; (PP)ROM - (Premature prelabor) rupture of membranes

Table A.7 | Neonatal sepsis related to interval between rupture of membranes and delivery (36 weeks) 


\section{LIST OF CO-AUTHORS}

Names and current affiliations of co-authors

Bettina MC Akerboom, MD, Dept. Obstetrics and Gynaecology, Albert Schweitzer Hospital, Dordrecht, the Netherlands

Birgit Arabin, MD PhD, Dept. Obstetrics and Gynaecology, Universitätsklinikum, Marburg, Germany

J (Hans) J van Beek, MD PhD, Dept. Obstetrics and Gynaecology, VieCuri Medical Centre, Venlo, the Netherlands

Denise Bijlenga, MD PhD, PsyQ, The Hague, the Netherlands

Kitty WM Bloemenkamp, MD PhD, Dept. Obstetrics and Gynaecology, Leiden University Medical Centre, Leiden, the Netherlands

Jan B Derks, MD PhD, Dept. Obstetrics and Gynaecology, University Medical Centre, Utrecht, the Netherlands

J (Hans) J Duvekot, MD PhD, Erasmus University Medical Centre, Rotterdam, the Netherlands

Maureen TM Franssen, MD PhD, Dept. Obstetrics and Gynaecology, University Medical Centre Groningen, Groningen, the Netherlands

Mariette Groenewout, MD PhD, Dept. Obstetrics and Gynaecology, University Medical Centre Groningen, Groningen, the Netherlands

Christianne JM de Groot, MD PhD, Dept. Obstetrics and Gynaecology, VU Medical Centre, Amsterdam, the Netherlands

Monique C Haak, MD PhD, Dept. Obstetrics and Gynaecology, Leiden University Hospital, Leiden, the Netherlands

Jantien L van der Heyden, MD, Dept. Obstetrics and Gynaecology, Maastricht University Medical Centre, Maastricht, the Netherlands

Michael M Kars, MD, Dept. Obstetrics and Gynaecology, St Antonieus Hospital, Nieuwegein, the Netherlands

Sander van Kuijk, MSc, Dept. Epidemiology, Maastricht University Medical Centre, Maastricht, the Netherlands

Simone Kuppens, MD PhD, Dept. Obstetrics and Gynaecology, Catharina Hospital, Eindhoven, the Netherlands

Anneke Kwee, MD PhD, Dept. Obstetrics and Gynaecology, University Medical Centre, Utrecht, the Netherlands

Rafli van de Laar, MD, Dept. Obstetrics and Gynaecology, St. Radboud University Medical Centre, Nijmegen, the Netherlands

Jan W de Leeuw, MD PhD, Dept. Obstetrics and Gynaecology, Ikazia Hospital, Rotterdam, the Netherlands

Aren J van Loon, MD PhD, Dept. Obstetrics and Gynaecology, Martini Hospital, Groningen, the Netherlands

Gerald D Mantel, MD, Dept. Obstetrics and Gynaecology, Isala Klinieken, Zwolle, the Netherlands

Marjo JGJ van Melick, MD, Dept. Obstetrics and Gynaecology, Maastricht University Medical Centre, Maastricht, the Netherlands

Godfried CH Metz, MD, Dept. Obstetrics and Gynaecology, Ikazia Hospital, Rotterdam, the Netherlands 
Ben Willem J Mol, MD PhD, Dept. Obstetrics and Gynaecology, Academic Medical Centre, Amsterdam, the Netherlands

Jan FM Molkenboer, MD PhD, Dept. Obstetrics and Gynaecology, Spaarne Hospital, Hoofddorp, the Netherlands

Rob Moonen, MD PhD, Dept. Paediatrics, Atrium Medical Centre, Heerlen, the Netherlands

A (Twan) LM Mulder, MD, PhD, Dept. Paediatrics, Maastricht University Medical Centre, Maastricht, the Netherlands

Jan G Nijhuis, MD PhD, Dept. Obstetrics and Gynaecology, Maastricht University Medical Centre, Maastricht, the Netherlands

S Guid Oei, MD PhD, Dept. Obstetrics and Gynaecology, Maxima Medical Centre, Veldhoven, the Netherlands

Brent C Opmeer, MSc PhD, Dept. Clinical Epidemiology, Biostatistics and Bioinformatics, Academic Medical Centre, Amsterdam, the Netherlands

Mariëlle G van Pampus, MD PhD, Dept. Obstetrics and Gynaecology, Onze Lieve Vrouwen Gasthuis, Amsterdam, the Netherlands

Dimitri NM Papatsonis, MD PhD, Dept. Obstetrics and Gynaecology, Amphia Hospital, Breda, the Netherlands

Paula JM Pernet, MD, Dept. Obstetrics and Gynaecology, Kennemer Gasthuis, Haarlem, the Netherlands

Martina M Porath, MD PhD, Dept. Obstetrics and Gynaecology, Maxima Medical Centre, Veldhoven, the Netherlands

Anita CJ Ravelli, MSc PhD, Dept. Medical Informatics, Academic Medical Centre, Amsterdam, the Netherlands

Jelle M Schaaf, MD PhD, Dept. Obstetrics and Gynaecology, Academic Medical Centre, Amsterdam, the Netherlands

H (Liesbeth) CJ Scheepers, MD PhD, Dept. Obstetrics and Gynaecology, Maastricht University Medical Centre, Maastricht, the Netherlands

Marko Sikkema, MD PhD, Dept. Obstetrics and Gynaecology, Twenteborg Hospital, Almelo, the Netherlands

Luc Smits, MSc PhD, Dept. Epidemiology, Maastricht University Medical Centre, Maastricht, the Netherlands

A (Stijn) SP van Teeffelen, MD, Dept. Obstetrics and Gynaecology, Maastricht University Medical Centre, Maasticht, the Netherlands

Sylvia MC Vijgen, MSc, Dept. Obstetrics and Gynaecology, Academic Medical Centre, Amsterdam, the Netherlands

Martin JN Weinans, MD PhD, Dept. Obstetrics and Gynaecology, Gelderse Vallei Hospital, Ede, the Nehtherlands

Carl P Weiner, MD PhD, Dept. Obstetrics and Gynaecology, University of Kansas Medical Centre, Kansas City, Kansas, United States of America

Wim J van Wijngaarden, MD PhD,Dept. Obstetrics and Gynaecology, Bronovo Hospital, the Hague, the Netherlands

Hajo IJ Wildschut, MD PhD, Dept. Obstetrics and Gynaecology, Westfriesgasthuis, Hoorn, the Netherlands

Christine Willekes, MD PhD, Dept. Obstetrics and Gynaecology, Maastricht University 
Medical Centre, Maastricht, the Netherlands

Mallory D Woiski, MD, Dept. Obstetrics and Gynaecology, St. Raboud University Medical Centre, Nijmegen, the Netherlands

\section{PPROMEXIL COLLABORATORS}

List of local researchers (not co-authors) in participating hospitals during the PPROMEXIL trial

R. Aardenburg, Orbis Medical Centre, Sittard; E.S.A. van den Akker, Onze Lieve Vrouwen Gasthuis, Amsterdam; E. van Beek, Mesos Medical Centre, Utrecht; R.E. Bernardus, Tergooi Hospitals, Blaricum/Hilversum; H.A. Bremer, Reinier de Graaf Gasthuis, Delft; K. de Boer, Rijnstate Hospital, Arnhem; J. T. J. Brons, Medical Spectrum Twente, Enschede; J.M. Burggraaff, Scheper Hospital, Emmen; M. Buimer, Westfries Gasthuis, Hoorn, F. Delemarre, Elkerliek Hospital, Helmond; B. Dijkman, BovenIJ Hospital, Amsterdam; J.P.R. Doornbos, Zaans Medical Centre, Zaandam; I.M.A. van Dooren, Sint Jans Gasthuis, Weert; A. Drogtrop, TweeSteden Hospital, Tilburg; J. Friederich, Gemini Hospital, Den Helder; N. van Gemund, Sint Franciscus Gasthuis, Rotterdam; A. van Ginkel, Hospital Zevenaar, Zevenaar; I.M. de Graaf, Spaarne Hospital, Hoofddorp; M.J.C.P. Hanssen, Bethesda Hospital, Hoogeveen; M.Heres, Lucas Andreas Hospital, Amsterdam; A.J.M. Huisjes, Gelre Hospital, Apeldoorn; M. van Huizen, Haga/Leyenburg Hospital, The Hague; G. Kleiverda, Flevo Hospital, Almere; R. Kokm Bernhoven Hospital, Veghel/Oss; A. Koops, Wilhelmina Hospital, Assen; C.A. van Meir, Groene Hart Hospital, Gouda, C.M. van Oirschot, Sint Elisabeth Hospital, Tilburg; D. Perquin, Medical Centre Leeuwarden, Leeuwarden; J.E. van de Riet, Antonius Hospital, Sneek; R.J.P. Rijnders, Jeroen Bosch Hospital, Den Bosch; F. Roumen, Atrium Medical Centre, Heerlen; P. van der Salm, Meander Medical Centre, Amersfoort; N.W.E. Schuitemaker, Diakonessen Hospital, Utrecht; R. Stigter, Deventer Hospital, Deventer; J. Wilpshaar, Nij Smellinghe, Drachten; A.C. de Wit, Maas Hospital, Boxmeer

\section{DUTCH RESEARCH CONSORTIUM}

Cornelieke vd Beek, Cynthia Crul, Zelda van Dijk, Maya Kruijt, Tessa Wiegerinck

\section{RESEARCH NURSES}

List of research nurses who are working for of have been working for the Dutch Consotrium from start of the PPROMEXIL trial until the end of 2011

Jannet Bakker (Cluster Amsterdam), Birgit van der Goes (Cluster Amsterdam), Sita Kleiterp (Cluster Amsterdam), Sabine Logtenberg (Cluster Amsterdam), Ageeth Rosman (Cluster Amsterdam), Viki Verfaille (Cluster Amsterdam), Corinne van de Griendt (Cluster Brabant), Lidewijde Jongmans (Cluster Brabant), Marieke Linders (Cluster Brabant), Kristel Slobben (Cluster Brabant), Corine Verhoeven (Cluster Brabant), Brenda Quak (Cluster Brabant), Ineke Hamming (Cluster Groningen), José Keurentjes (Cluster Groningen), Lida UIkeman (Cluster Groningen), Marianne van der Hel (Cluster Leiden), Clara Kolster (Cluster Leiden), Marjolein Verhart (Cluster Leiden), Inge Boot (Cluster Limburg), Monique Braken-Florax (Cluster Limburg), Katinka Burgers (Cluster Limburg), Kim Notten (Cluster Limburg), Vivian Ramaekers (Cluster Limburg), Brenda Spauwen (Cluster Limburg), Nathalie 
Teeuwen (Cluster Limburg), Jolanda Willems-Robberts (Cluster Limburg), Wilma Meijer (Cluster Nijmegen), Hanneke van de Rijt (Cluster Nijmegen), Gerard Zijderveld (Cluster Nijmegen), Joyce Cantineau (Cluster Rotterdam), Wilma Keller (Cluster Rotterdam), Nanny Konings (Cluster Rotterdam), Nelly le Noble (Cluster Rotterdam), Joke van Rhee (Cluster Rotterdam), Titia Winter Cluster Rotterdam), David Borman (Cluster Utrecht), Coby van Dam-Bouwens (Cluster Utrecht), Kathy van den Berg-Swart (Cluster Utrecht), Maartje de Reus (Cluster Utrecht), Ina Smallegange (Cluster Utrecht), Michelle Westerhuis (Cluster Utrecht), Sylvia Arendsen (Cluster Twente), José Krabbe (Cluster Twente), Edwin Lubbers (Cluster Twente), Maria Marang (Cluster Twente), Marleen Nahuis (Cluster Twente), Ingrid Volker (Cluster Twente), Diana Lutjes (Cluster Zwolle)

\section{ACKNOWLEDGMENT}

I would like to thank Julia Spaan, Marta Jozwiak, Drora Bijlenga Goldstein, Martina Porath, Violetta Huijs-Spiewanek, L. Tania Caballero Vega, for their help in aquiring manuscripts and translating manuscripts for chapter 2 and chapter 4 


\section{REFERENCELIST}

1. Furman B, Shoham-Vardi I, Bashiri A, Erez O, Mazor M. Clinical significance and outcome of preterm prelabor rupture of membranes: population-based study. Eur J Obstet Gynecol Reprod Biol 2000;92:209-16.

2. Mercer BM. Preterm premature rupture of the membranes. Obstet Gynecol 2003;101:178-93.

3. Hannah ME, Ohlsson A, Farine D, et al. Induction of labor compared with expectant management for prelabor rupture of the membranes at term. TERMPROM Study Group. N Engl J Med 1996;334:1005-10.

4. Ottervanger HP, Keirse MJ, Smit W, Holm JP. Controlled comparison of induction versus expectant care for prelabor rupture of the membranes at term. $J$ Perinat Med 1996;24:237-42.

5. Nederlandse Vereniging voor Obsterie en Gynaecologie. [Breken van de vliezen voor het begin van de baring]. Available at: http://nvogdocumenten.nl/index.php?pagina/ richtlijn/item/pagina.php\&richtlijn_id564.

6. Arias F, Tomich P. Etiology and outcome of low birth weight and preterm infants. Obstetrics and Gynecology 1982;60:277-81.

7. Gonen R, Hannah ME, Milligan JE. Does prolonged preterm premature rupture of the membranes predispose to abruptio placentae? Obstetrics and Gynecology 1989;74:347-50.

8. Major CA, de Veciana M, Lewis DF, Morgan MA. Preterm premature rupture of membranes and abruptio placentae: is there an association between these pregnancy complications? Am J Obstet Gynecol 1995;172:672-6.

9. Goldenberg RL, Nelson KG, Davis RO, Koski J. Delay in delivery: influence of gestational age and the duration of delay on perinatal outcome. Obstet Gynecol 1984;64:4804.

10. Friedman ML, McElin TW. Diagnosis of ruptured fetal membranes. Clinical study and review of the literature. Am J Obstet Gynecol 1969;104:544-50.

11. de Haan HH, Offermans PM, Smits F, Schouten HJ, Peeters LL. Value of the fern test to confirm or reject the diagnosis of ruptured membranes is modest in nonlaboring women presenting with nonspecific vaginal fluid loss. Am J Perinatol 1994;11:46-50.

12. Erdemoglu E, Mungan T. Significance of detecting insulin-like growth factor binding protein-1 in cervicovaginal secretions: comparison with nitrazine test and amniotic fluid volume assessment. Acta Obstet Gynecol Scand 2004;83:622-6.

13. Gorodeski IG, Haimovitz L, Bahari CM. Reevaluation of the $\mathrm{pH}$, ferning and nile blue sulphate staining methods in pregnant women with premature rupture of the fetal membranes. J Perinat Med 1982;10:286-92.

14. Royal College of Obstetricians and Gynaecologists. Gynaecological Examinations: Guidelines for Specialist Practice. London: RCOG; 2002.

15. American College of Obstetricians and Gynecology. ACOG practice bulletin no. 80: premature rupture of membranes. Clinical management guidelines for obstetriciangynecologists. Obstet Gynecol 2007;109:1007-19.

16. Royal College of Obstetricians and Gynaecologists. Preterm prelabour rupture of membranes. Guideline no. 44; 2006. Available at: http://www.rcog.org.uk/files/ rcog-corp/uploaded-files/GT44PretermPrelabourRupture2006.pfd.

17. Buchanan S, Crowther C, Morris J. Preterm prelabour rupture of the membranes: a survey of current practice. Aust N Z J Obstet Gynaecol 2004;44:400-3.

18. Smith G, Rafuse C, Anand N, et al. Prevalence, management, and outcomes of preterm prelabour rupture of the membranes of women in Canada. J Obstet Gynaecol Can 2005;27:547-53.

19. Buchanan SL, Crowther CA, Levett KM, Middleton P, Morris J. Planned early birth versus expectant management for women with preterm prelabour rupture of membranes prior to 37 weeks' gestation for improving pregnancy outcome. Cochrane Database Syst Rev 2010:CD004735. 
20. Cox SM, Leveno KJ. Intentional delivery versus expectant management with preterm ruptured membranes at 30-34 weeks' gestation. Obstet Gynecol 1995;86:875-9.

21. Garite TJ, Freeman RK, Linzey EM, Braly PS, Dorchester WL. Prospective randomized study of corticosteroids in the management of premature rupture of the membranes and the premature gestation. Am J Obstet Gynecol 1981;141:508-15.

22. lams JD, Talbert ML, Barrows H, Sachs L. Management of preterm prematurely ruptured membranes: a prospective randomized comparison of observation versus use of steroids and timed delivery. Am J Obstet Gynecol 1985;151:32-8.

23. Mercer BM, Crocker LG, Boe NM, Sibai BM. Induction versus expectant management in premature rupture of the membranes with mature amniotic fluid at 32 to 36 weeks: a randomized trial. Am J Obstet Gynecol 1993;169:775-82.

24. Naef RW, 3rd, Allbert JR, Ross EL, Weber BM, Martin RW, Morrison JC. Premature rupture of membranes at 34 to 37 weeks' gestation: aggressive versus conservative management. Am J Obstet Gynecol 1998;178:126-30.

25. Nelson LH, Meis PJ, Hatjis CG, Ernest JM, Dillard R, Schey HM. Premature rupture of membranes: a prospective, randomized evaluation of steroids, latent phase, and expectant management. Obstet Gynecol 1985;66:55-8.

26. Spinnato JA, Shaver DC, Bray EM, Lipshitz J. Preterm premature rupture of the membranes with fetal pulmonary maturity present: a prospective study. Obstet Gynecol 1987;69:196-201.

27. van der Ham DP, Nijhuis JG, Mol BW, et al. Induction of labour versus expectant management in women with preterm prelabour rupture of membranes between 34 and 37 weeks (the PPROMEXIL-trial). BMC Pregnancy Childbirth 2007;7:11.

28. Gunn GC, Mishell DR, Jr., Morton DG. Premature rupture of the fetal membranes. A review. Am J Obstet Gynecol 1970;106:469-83.

29. Mead PB. Management of the patient with premature rupture of the membranes. Clin Perinatol 1980; 7:243-55.

30. Cox SM, Williams ML, Leveno KJ. The natural history of preterm ruptured membranes: what to expect of expectant management. Obstet Gynecol 1988;71:558-62.

31. Gibbs RS, Blanco JD. Premature rupture of the membranes. Obstet Gynecol 1982;60:671-9.

32. Mercer BM, Goldenberg RL, Meis PJ, et al. The Preterm Prediction Study: prediction of preterm premature rupture of membranes through clinical findings and ancillary testing. The National Institute of Child Health and Human Development MaternalFetal Medicine Units Network. Am J Obstet Gynecol 2000;183:738-45.

33. Baptisti A. Chemical test for the determination of ruptured membranes. Am J Obstet Gynecol 1938;87:688-90.

34. Ni CY, Jia WX, Yi WM, Feng LH, Yu LZ. Practicability of using vaginal fluid markers in detecting premature rupture of membranes. Ann Clin Biochem 2003;40:542-5.

35. Rochelson BL, Richardson DA, Macri JN. Rapid assay - possible application in the diagnosis of premature rupture of the membranes. Obstet and Gynecol 1983;62:4148.

36. Anai T, Tanaka Y, Hirota Y, Miyakawa I. Vaginal fluid hCG levels for detecting premature rupture of membranes. Obstet Gynecol 1997;89:261-4.

37. Kim YH, Park YW, Kwon HS, Kwon JY, Kim BJ. Vaginal fluid beta-human chorionic gonadotropin level in the diagnosis of premature rupture of membranes. Acta Obstet Gynecol Scand 2005;84:802-5.

38. Gurbuz A, Karateke A, Kabaca C. Vaginal fluid creatinine in premature rupture of membranes. Int J Gynaecol Obstet 2004;85:270-1.

39. Kafali $\mathrm{H}, \mathrm{Oksuzler} \mathrm{C}$. Vaginal fluid urea and creatinine in diagnosis of premature rupture of membranes. Archives of Gynecology and Obstetrics 2007;275:157-60.

40. Elmfors B, Tryding N, Tufvesson G. The diagnosis of ruptured fetal membranes by measurement of the diamine oxidase (DAO) activity in vaginal fluid. J Obstet Gynaecol Br Commonw 1974;81:361-2.

41. Gaucherand P, Salle B, Sergeant P, et al. Comparative study of three vaginal markers 
of the premature rupture of membranes. Insulin like growth factor binding protein 1 diamine-oxidase pH. Acta Obstet Gynecol Scand 1997;76:536-40.

42. Eriksen NL, Parisi VM, Daoust S, Flamm B, Garite TJ, Cox SM. Fetal fibronectin: a method for detecting the presence of amniotic fluid. Obstet Gynecol 1992;80:451-4.

43. Hellemans $P$, Verdonk $P$, Baekelandt $M$, Joostens $M$, Francx M, Gerris J. Preliminary results with the use of the ROM-check immunoassay in the early detection of rupture of the amniotic membranes. Eur J Obstet Gynecol Reprod Biol 1992;43:173-9.

44. Gorodeski IG, Paz M, Insler V, Fishel J. Diagnosis of rupture of the fetal membranes by glucose and fructose measurements. Obstet Gynecol 1979;53:611-6.

45. Jeurgens-Borst AJ, Bekkers RL, Sporken JM, van den Berg PP. Use of insulin like growth factor binding protein-1 in the diagnosis of ruptured fetal membranes. Eur J Obstet Gynecol Reprod Biol 2002;102:11-4.

46. Kubota T, Takeuchi $\mathrm{H}$. Evaluation of insulin-like growth factor binding protein-1 as a diagnostic tool for rupture of the membranes. J Obstet Gynaecol Res 1998;24:411-7.

47. Wiberg-Itzel E, Cnattingius S, Nordstrom L. Lactate determination in vaginal fluids: a new method in the diagnosis of prelabour rupture of membranes. Bjog 2005;112:754-8.

48. Wiberg-Itzel E, Pettersson H, Cnattingius S, Nordstrom L. Prediction of time to spontaneous onset of labour with lactate concentration in vaginal fluid in women with suspected preterm prelabour rupture of the membranes. Bjog 2009;116:62-6.

49. Gariepy G, Vauclair R. Study of ruptured fetal membranes by Nile blue sulfate staining. Acta Cytol 1969;13:154-7.

50. Bornstein J, Geva A, Solt I, et al. Nonintrusive diagnosis of premature ruptured amniotic membranes using a novel polymer. Am J Perinatol 2006;23:351-4.

51. Bornstein J, Ohel G, Sorokin Y, et al. Effectiveness of a novel home-based testing device for the detection of rupture of membranes. Am J Perinatol 2009;26:45-50.

52. Meyer BA, Gonik B, Creasy RK. Evaluation of phenazopyridine hydrochloride as a tool in the diagnosis of premature rupture of the membranes. Am J Perinatol 1991;8:2979.

53. Cousins LM, Smok DP, Lovett SM, Poeltler DM. AmniSure placental alpha microglobulin-1 rapid immunoassay versus standard diagnostic methods for detection of rupture of membranes. Am J Perinatol 2005;22:317-20.

54. Lee SE, Park JS, Norwitz ER, Kim KW, Park HS, Jun JK. Measurement of placental alpha-microglobulin-1 in cervicovaginal discharge to diagnose rupture of membranes. Obstet Gynecol 2007;109:634-40.

55. Buyukbayrak EE, Turan C, Unal O, Dansuk R, Cengizoglu B. Diagnostic power of the vaginal washing-fluid prolactin assay as an alternative method for the diagnosis of premature rupture of membranes. J Matern Fetal Neonatal Med 2004;15:120-5.

56. Koninckx PR, Trappeniers $H$, Van Assche FA. Prolactin concentration in vaginal fluid: a new method for diagnosing ruptured membranes. Br J Obstet Gynaecol 1981;88:607-10.

57. Cowett RM, Hakanson DO, Kocon RW, Oh W. Untoward neonatal effect of intraamniotic administration of methylene blue. Obstet Gynecol 1976;48:74S-5S.

58. Fujimoto S, Kishida T, Sagawa T, et al. Clinical usefulness of the dye-injection method for diagnosing premature rupture of the membranes in equivocal cases. J Obstet Gynaecol 1995;21:215-20.

59. McEnerney JK, McEnerney LN. Unfavorable neonatal outcome after intraamniotic injection of methylene blue. Obstet Gynecol 1983;61:35S-7S.

60. Smith RP. A technic for the detection of rupture of the membranes. A review and preliminary report. Obstet Gynecol 1976;48:172-6.

61. Bossuyt PM, Reitsma JB, Bruns DE, et al. Towards complete and accurate reporting of studies of diagnostic accuracy: the STARD initiative. Bmj 2003;326:41-4.

62. Deville WL, Buntinx F, Bouter $L M$, et al. Conducting systematic reviews of diagnostic studies: didactic guidelines. BMC Med Res Methodol 2002;2:9.

63. Rutjes AW, Reitsma JB, Coomarasamy A, Khan KS, Bossuyt PM. Evaluation of diagnos- 
tic tests when there is no gold standard. A review of methods. Health Technol Assess 2007;11:iii, ix-51.

64. Whiting P, Rutjes AW, Reitsma JB, Bossuyt PM, Kleijnen J. The development of QUADAS: a tool for the quality assessment of studies of diagnostic accuracy included in systematic reviews. BMC Med Res Methodol 2003;3:25.

65. Averette HE, Hammond DO, Cerha HT, Neri AS, Ferguson JH. Endocrine colpocytology and cytodiagnosis of ruptured fetal membranes. Jama 1967;202:640-4.

66. Bank CM, Offermans JP, Gijzen AH, Smits F, van Dieijen-Visser MP, Brombacher PJ. Diamine oxidase activity in amniotic fluid for diagnosis of ruptured membranes. Eur J Clin Chem Clin Biochem 1991;29:743-8.

67. Beckmann MW, Wiegratz I, Dereser MM, Baier P, Born HJ. [Diagnosis of rupture of fetal membranes: comparison of vaginal detection of fetal fibronectin and intra-amnion injection of indigo carmine]. Geburtshilfe Frauenheilkd 1993;53:86-91.

68. Mulhair L, Carter J, Poston L, Seed P, Briley A. Prospective cohort study investigating the reliability of the AmnioSense(trademark) method for detection of spontaneous rupture of membranes. Bjog 2009;116:313-8.

69. Watanabe T, Minakami H, Itoi H, Sato I, Sakata Y, Tamada T. Evaluation of latex agglutination test for alpha-fetoprotein in diagnosing rupture of fetal membranes. Gynecol Obstet Invest 1995;39:15-8.

70. Yamada H, Kishida T, Negishi H, et al. Comparison of an improved AFP kit with the intra-amniotic PSP dye-injection method in equivocal cases of preterm premature rupture of the fetal membranes. J Obstet Gynaecol Res 1997;23:307-11.

71. Ladfors L, Mattsson LA, Eriksson M, Fall O. Is a speculum examination sufficient for excluding the diagnosis of ruptured fetal membranes? Acta Obstet Gynecol Scand 1997;76:739-42.

72. van der Ham DP, van Melick MJ, Smits L, et al. Methods for the diagnosis of rupture of the fetal membranes in equivocal cases: a systematic review. Eur J Obstet Gynecol Reprod Biol 2011;157:123-7.

73. Simhan HN, Canavan TP. Preterm premature rupture of membranes: diagnosis, evaluation and management strategies. Bjog 2005;112 Suppl 1:32-7.

74. Silva E, Martinez J. Diagnosing ROM: a comparison of the gold standard, indigo carmine amnioinfusion, to the rapid immunoassay, the AmniSure ROM test. J Perinat Med 2009;37:956.

75. Di Renzo GC, Roura LC, Facchinetti F, et al. Guidelines for the management of spontaneous preterm labor: identification of spontaneous preterm labor, diagnosis of preterm premature rupture of membranes, and preventive tools for preterm birth. J Matern Fetal Neonal 2011;24:659-67.

76. Riboni F, Vitulo A, Plebani M, Dell'avanzo M, Battagliarin G, Paternoster D. Combination of biochemical markers in predicting pre-term delivery. Archives of Gynecology and Obstetrics 2012;285:61-6.

77. Rutanen EM, Karkkainen TH, Lehtovirta J, Uotila JT, Hinkula MK, Hartikainen AL. Evaluation of a rapid strip test for insulin-like growth factor binding protein-1 in the diagnosis of ruptured fetal membranes. Clin Chim Acta 1996;253:91-101.

78. Abdelazim IA, Makhlouf HH. Placental alpha microglobulin-1 (AmniSure((R)) test) for detection of premature rupture of fetal membranes. Archives of Gynecology and Obstetrics 2012;285:985-9.

79. Albayrak M, Ozdemir I, Koc O, Ankarali H, Ozen O. Comparison of the diagnostic efficacy of the two rapid bedside immunoassays and combined clinical conventional diagnosis in prelabour rupture of membranes. Eur J Obstet Gynecol Reprod Biol 2011;158:179-82.

80. Birkenmaier A, Ries JJ, Kuhle J, Burki N, Lapaire O, Hosli I. Placental alpha-microglobulin-1 to detect uncertain rupture of membranes in a European cohort of pregnancies. Archives of Gynecology and Obstetrics 2011.

81. Neil PR, Wallace EM. Is Amnisure(R) useful in the management of women with prelabour rupture of the membranes? Aust N Z J Obstet Gynaecol 2010;50:534-8. 
82. Pollet-Villard M, Cartier R, Gaucherand P, Doret M. Detection of placental alpha microglobulin-1 versus insulin-like growth factor-binding protein-1 in amniotic fluid at term: a comparative study. Am J Perinatol 2011;28:489-94.

83. Tagore $\mathrm{S}, \mathrm{Kwek} \mathrm{K}$. Comparative analysis of insulin-like growth factor binding protein-1 (IGFBP-1), placental alpha-microglobulin-1 (PAMG-1) and nitrazine test to diagnose premature rupture of membranes in pregnancy. J Perinat Med 2010;38:609-12.

84. Wang T, Zhou R, Zhang L, et al. Proteins in leaked amniotic fluid as biomarkers diagnostic for prelabor rupture of membranes. Proteomics Clin Appl 2011;5:415-21.

85. Zanjani MS, Haghighi L. Vaginal fluid creatinine for the detection of premature rupture of membranes.J Obstet Gynaecol Research 2012;38:505-8.

86. Lee SM, Lee J, Seong HS, et al. The clinical significance of a positive Amnisure test in women with term labor with intact membranes. J Matern Fetal Neona 2009;22:30510.

87. Cooper S, Lange I, Wood S, Tang S, Miller L, Ross S. Diagnostic accuracy of rapid phIGFBP-I assay for predicting preterm labor in symptomatic patients. $J$ Perinatol 2012;32:460-5.

88. Lee J, Lee SM, Oh KJ, Park CW, Jun JK, Yoon BH. Fragmented forms of insulin-like growth factor binding protein-1 in amniotic fluid of patients with preterm labor and intact membranes. Reprod Sci 2011;18:842-9.

89. van der Ham DP, Vijgen SM, Nijhuis JG, et al. Induction of labor versus expectant management in women with preterm prelabor rupture of membranes between 34 and 37 weeks: a randomized controlled trial. PLoS Medicine 2012;9:e1001208.

90. Goldenberg RL. The management of preterm labor. Obstet Gynecol 2002;100:102037.

91. Merenstein GB, Weisman LE. Premature rupture of the membranes: neonatal consequences. Semin Perinatol 1996;20:375-80.

92. Dodds WG, lams JD. Maternal C-reactive protein and preterm labor. J Reprod Med 1987;32:527-30.

93. Hawrylyshyn P, Bernstein P, Milligan JE, Soldin S, Pollard A, Papsin FR. Premature rupture of membranes: the role of C-reactive protein in the prediction of chorioamnionitis. Am J Obstet Gynecol 1983;147:240-6.

94. Ibarra Chavarria V, Sanhueza Smith P, Mota Gonzalez M, del Rey Pineda G, Karchmer $\mathrm{S}$. [C-reactive protein as early marker of chorioamnionitis in premature rupture of membranes]. Ginecol Obstet Mex 1989;57:203-8.

95. Romem Y, Artal R. C-reactive protein as a predictor for chorioamnionitis in cases of premature rupture of the membranes. Am J Obstet Gynecol 1984;150:546-50.

96. Evans MI, Hajj SN, Devoe LD, Angerman NS, Moawad AH. C-reactive protein as a predictor of infectious morbidity with premature rupture of membranes. Am J Obstet Gynecol 1980;138:648-52.

97. Trochez-Martinez RD, Smith P, Lamont RF. Use of C-reactive protein as a predictor of chorioamnionitis in preterm prelabour rupture of membranes: a systematic review. Bjog 2007;114:796-801.

98. Sereepapong W, Limpongsanurak S, Triratanachat S, Wannakrairot P, Charuruks N, Krailadsiri P. The role of maternal serum C-reactive protein and white blood cell count in the prediction of chorioamnionitis in women with premature rupture of membranes. J Med Assoc Thai 2001;84 Suppl 1:S360-6.

99. Kurki T, Teramo K, Ylikorkala O, Paavonen J. C-reactive protein in preterm premature rupture of the membranes. Arch Gynecol Obstet 1990;247:31-7.

100. Yoon BH, Jun JK, Park KH, Syn HC, Gomez R, Romero R. Serum C-reactive protein, white blood cell count, and amniotic fluid white blood cell count in women with preterm premature rupture of membranes. Obstet Gynecol 1996;88:1034-40.

101. Bankowska EM, Leibschang J, Pawlowska A. [Usefulness of determination of granulocyte elastase plasma level, c-reactive protein and white blood cell count in prediction in intrauterine infection in pregnant women after PROM]. Ginekol Pol 2003; 74:1037-43. 
102. Nowak M, Oszukowski P, Szpakowski M, Malinowski A, Maciolek-Blewniewska G. [Intrauterine infections. I. The role of C-reactive protein, white blood cell count and erythrocyte sedimentation rate in pregnant women in the detection of intrauterine infection after preliminary rupture of membranes]. Ginekol Pol 1998;69:615-22.

103. Lieman JM, Brumfield CG, Carlo W, Ramsey PS. Preterm premature rupture of membranes: is there an optimal gestational age for delivery? Obstet Gynecol 2005;105:127.

104. Deeks JJ. Systematic reviews in health care: Systematic reviews of evaluations of diagnostic and screening tests. Bmj 2001;323:157-62.

105. Reitsma JB, Glas AS, Rutjes AW, Scholten RJ, Bossuyt PM, Zwinderman AH. Bivariate analysis of sensitivity and specificity produces informative summary measures in diagnostic reviews. J Clin Epidemiol 2005;58:982-90.

106. van Houwelingen HC, Arends LR, Stijnen T. Advanced methods in meta-analysis: multivariate approach and meta-regression. Stat Med 2002;21:589-624.

107. Van Houwelingen HC, Zwinderman KH, Stijnen T. A bivariate approach to meta-analysis. Stat Med 1993;12:2273-84.

108. He JP. [C-reactive protein and acute-phase proteins in monitoring infection caused by premature rupture of membranes]. Zhonghua Fu Chan Ke Za Zhi 1990;25:98101, 25.

109. Tiufekchieva E. [C-reactive protein as an index of amniotic infection in premature rupture of the amniotic sac]. Akush Ginekol (Sofiia) 1985;24:35-41.

110. Gonik B, Weiner C, James D, Steer P, eds. High risk pregnancy management options. 3rd ed. Philadelphia: W.B. Saunders Company; 2005.

111. Ismail MA, Zinaman MJ, Lowensohn RI, Moawad AH. The significance of C-reactive protein levels in women with premature rupture of membranes. Am J Obstet Gynecol 1985;151:541-4.

112. Berardi JC, Hutin S, Godard J, Madinier V, Delanete A, Berardi-Grassias L. [The value of $C$-reactive protein in the detection of chorioamnionitis in cases of premature rupture of membranes]. Rev Fr Gynecol Obstet 1991;86:229-32.

113. Fisk NM, Fysh J, Child AG, Gatenby PA, Jeffery H, Bradfield AH. Is C-reactive protein really useful in preterm premature rupture of the membranes? Bjog 1987;94:115964.

114. Farb HF, Arnesen M, Geistler P, Knox GE. C-reactive protein with premature rupture of membranes and premature labor. Obstet Gynecol 1983;62:49-51.

115. Ramsey PS, Lieman JM, Brumfield CG, Carlo W. Chorioamnionitis increases neonatal morbidity in pregnancies complicated by preterm premature rupture of membranes. Am J Obstet Gynecol 2005;192:1162-6.

116. Williams $\mathrm{H}$, Jeffery $\mathrm{H}$. The incidence of histological chorioamnionitis in IVF/GIFT preterm births. Aust N Z J Obstet Gynaecol 1994;34:480-3.

117. Lewis DF, Futayyeh S, Towers CV, Asrat T, Edwards MS, Brooks GG. Preterm delivery from 34 to 37 weeks of gestation: is respiratory distress syndrome a problem? Am J Obstet Gynecol 1996;174:525-8.

118. Neerhof MG, Cravello C, Haney El, Silver RK. Timing of labor induction after premature rupture of membranes between 32 and 36 weeks' gestation. Am J Obstet Gynecol 1999;180:349-52.

119. Garite TJ. Management of premature rupture of membranes. Clinics in perinatology 2001;28:837-47.

120. Hartling L, Chari R, Friesen C, Vandermeer B, Lacaze-Masmonteil T. A systematic review of intentional delivery in women with preterm prelabor rupture of membranes. J Matern Fetal Neonatal Med 2006;19:177-87.

121. Goldstein B, Giroir B, Randolph A. International pediatric sepsis consensus conference: definitions for sepsis and organ dysfunction in pediatrics. Pediatr Crit Care Med 2005;6:2-8.

122. Neu J. Necrotizing enterocolitis: the search for a unifying pathogenic theory leading to prevention. Pediatr Clin North Am 1996;43:409-32. 
123. Sarnat HB, Sarnat MS. Neonatal encephalopathy following fetal distress. A clinical and electroencephalographic study. Arch Neurol 1976;33:696-705.

124. Hays W. Statistics. New York: Holt, Rinehart and Winston; 1998.

125. Preterm Premature Rupture Of Membranes between 34 and 37 weeks: EXpecatant management versus Induction of Labour. http://www.controlled-trials.com/mrct/ trial/257003/PPROMEXIL

126. Clinical trial: Safety and Efficacy Study of Intentional Delivery in Women With Preterm and Prelabour Rupture of the Membranes. http://clinicaltrials.gov/ct/show/NC T00259519; ;sessionid=511720C5DF99149F8A22D293DBDF071E ?order=1.

127. A randomised controlled trial of immediate delivery versus expectant care in women with ruptured membranes close to term. http://www.controlled-trials.com/mrct/ trial/228805/PPROMT.

128. Morris JM, Roberts CL, Crowther CA, Buchanan SL, Henderson-Smart DJ, Salkeld G. Protocol for the immediate delivery versus expectant care of women with preterm prelabour rupture of the membranes close to term (PPROMT) Trial [ISRCTN44485060]. BMC Pregnancy Childbirth 2006;6:9.

129. ZonMw-Projectenpoort - Induction of labour versus expectant management in women with preterm premature rupture of membranes between 34 and 37 weeks. http://zonmw.collexis.net/projectsummary.asp?foreignid=94507212

130. Buchanan DD, F JM. A role for intravenous lidocaine in severe cancer-related neuropathic pain at the end-of-life. Support Care Cancer 2010;18:899-901.

131. Nederlandse Vereniging voor Obstetrie en Gynaecologie [Induction of labor]. http:// novog-documenten.nl/index.php?pagina=/richtlijn/item/pagina.php\&richtlijn_ id=689.

132. Review Manager (RevMan) In. 5.1 ed. Copenhagen: The Nordic Cochrane Centre, The Cochrane Collaboration; 2011.

133. Alexander JM, McIntire DM, Leveno KJ. Chorioamnionitis and the prognosis for term infants. Obstet Gynecol 1999;94:274-8.

134. Lau J, Magee F, Qiu Z, Hoube J, Von Dadelszen P, Lee SK. Chorioamnionitis with a fetal inflammatory response is associated with higher neonatal mortality, morbidity, and resource use than chorioamnionitis displaying a maternal inflammatory response only. Am J Obstet Gynecol 2005;193:708-13.

135. Leviton A, Allred EN, Kuban KC, et al. Microbiologic and histologic characteristics of the extremely preterm infant's placenta predict white matter damage and later cerebral palsy. the ELGAN study. Pediatr Res 2010;67:95-101.

136. O'Shea TM, Allred EN, Dammann O, et al. The ELGAN study of the brain and related disorders in extremely low gestational age newborns. Early Hum Dev 2009;85:71925.

137. Kayem G, Bernier-Dupreelle A, Goffinet F, Cabrol D, Haddad B. Active versus expectant management for preterm prelabor rupture of membranes at 34-36 weeks of completed gestation: comparison of maternal and neonatal outcomes. Acta Obstet Gynecol Scand 2010;89:776-81.

138. Hansen AK, Wisborg K, Uldbjerg N, Henriksen TB. Risk of respiratory morbidity in term infants delivered by elective caesarean section: cohort study. Bmj 2008;336:857.

139. Burns CM, Rutherford MA, Boardman JP, Cowan FM. Patterns of cerebral injury and neurodevelopmental outcomes after symptomatic neonatal hypoglycemia. Pediatrics 2008;122:65-74.

140. Lucas A, Morley R, Cole TJ. Adverse neurodevelopmental outcome of moderate neonatal hypoglycaemia. Bmj 1988;297:1304-8.

141. Shapiro SM. Bilirubin toxicity in the developing nervous system. Pediatr Neurol 2003;29:410-21.

142. Newman TB, Liljestrand P, Jeremy RJ, et al. Outcomes among newborns with total serum bilirubin levels of $25 \mathrm{mg}$ per deciliter or more. N Engl J Med 2006;354:1889900. 
143. MacKay DF, Smith GC, Dobbie R, Pell JP. Gestational age at delivery and special educational need: retrospective cohort study of 407,503 schoolchildren. PLoS Medicine 2010;7:e1000289.

144. Boers KE, Vijgen SM, Bijlenga D, et al. Induction versus expectant monitoring for intrauterine growth restriction at term: randomised equivalence trial (DIGITAT). Bmj 2010;341:c7087.

145. Koopmans CM, Bijlenga D, Groen H, et al. Induction of labour versus expectant monitoring for gestational hypertension or mild pre-eclampsia after 36 weeks' gestation (HYPITAT): a multicentre, open-label randomised controlled trial. Lancet 2009;374:979-88.

146. Mateus J, Fox K, Jain S, Latta R, Cohen J. Preterm premature rupture of membranes: clinical outcomes of late-preterm infants. Clin Pediatr (Phila) 2010;49:60-5.

147. Nederlandse Vereniging voor Obstetrie en Gynaecologie [Rupture of membranes before onset of labor]. http://nvog-documenten.nl/index.php?pagina=/richtlijn/pagina.php\&fSelectTG_62=75\&fSelectedSub=62\&fSelectedParent=75.)

148. van der Ham DP, van der Heyden JL, Opmeer BC, et al. Management of late-preterm premature rupture of membranes: the PPROMEXIL-2 trial. Am J Obstet Gynecol 2012. 276.e1-276.e10.

149. Morris J, Crowther C. A randomised controlled trial of immediate delivery versus expectant care in women with ruptured membranes close to term. ISRCTN 44485060. In: Australian National Health and Medical Research Council Royal North Shore Hospital, Australia, 2005.

150. The Netherlands Perinatal Registry. PRN-foundation. http://www.perinatreg.nl/ home_english.

151. Meray N, Reitsma JB, Ravelli AC, Bonsel GJ. Probabilistic record linkage is a valid and transparent tool to combine databases without a patient identification number. $J$ Clin Epidemiol 2007;60:883-91.

152. Tromp M, Ravelli AC, Meray N, Reitsma JB, Bonsel GJ. An efficient validation method of probabilistic record linkage including readmissions and twins. Methods Inf Med 2008;47:356-63.

153. Langenveld J, Ravelli AC, van Kaam AH, et al. Neonatal outcome of pregnancies complicated by hypertensive disorders between 34 and 37 weeks of gestation: a 7 year retrospective analysis of a national registry. Am J Obstet Gynecol. 2011 Dec;205(6):540.e1-7

154. Bell MJ, Ternberg JL, Feigin RD, et al. Neonatal necrotizing enterocolitis. Therapeutic decisions based upon clinical staging. Ann Surg 1978;187:1-7.

155. Papile LA, Burstein J, Burstein R, Koffler H. Incidence and evolution of subependymal and intraventricular hemorrhage: a study of infants with birth weights less than 1,500 gm. J Pediatr 1978;92:529-34.

156. de Vries LS, Eken P, Dubowitz LM. The spectrum of leukomalacia using cranial ultrasound. Behav Brain Res 1992;49:1-6.

157. Harrell FJ. Regression Modeling Strategies. New York: Springer; 2001.

158. Allison P. Missing Data. lowa: Sage; 2001.

159. Donders AR, van der Heijden GJ, Stijnen T, Moons KG. Review: a gentle introduction to imputation of missing values. J Clin Epidemiol 2006;59:1087-91.

160. Steyerberg EW. Clinical Prediction Models. New York: Springer; 2009.

161. Rubin D. Inference and missing data. Biometrika 1976;63:581-92.

162. Hanley JA, McNeil BJ. A method of comparing the areas under receiver operating characteristic curves derived from the same cases. Radiology 1983;148:839-43.

163. Steyerberg EW, Vickers AJ, Cook NR, et al. Assessing the performance of prediction models: a framework for traditional and novel measures. Epidemiology 2010;21:12838.

164. Sun GW, Shook TL, Kay GL. Inappropriate use of bivariable analysis to screen risk factors for use in multivariable analysis. J Clin Epidemiol 1996;49:907-16.

165. van de Laar R, van der Ham DP, Oei SG, Willekes C, Weiner CP, Mol BW. Accuracy 
of C-reactive protein determination in predicting chorioamnionitis and neonatal infection in pregnant women with premature rupture of membranes: a systematic review. Eur J Obstet Gynecol Reprod Biol 2009;147:124-9.

166. van der Heyden JL, van Teeffelen SS, Coolen AC, et al. Is it useful to measure C-reactive protein and leukocytes in patients with prelabor rupture of membranes? Am J Perinatol 2010;27:543-7.

167. Weston EJ, Pondo T, Lewis MM, et al. The burden of invasive early-onset neonatal sepsis in the United States, 2005-2008. Pediatr Infect Dis J 2011;30:937-41.

168. Sgro M, Shah PS, Campbell D, Tenuta A, Shivananda S, Lee SK. Early-onset neonatal sepsis: rate and organism pattern between 2003 and 2008. J Perinatol 2011;31:7948.

169. Drummond MF, Sculpher MJ, Torrance GW, O'Brien BJ, Stoddart GL. Methods for the Economic Evaluation of Health Care Programmes. Oxford: Oxford University Press; 2005.

170. Oostenbrink JB. Standardisation of costs: the Dutch Manual for Costing in Economic Evaluations. Pharmacoeconomics 2009;20:443-54.

171. Van der Kuy A. Pharmacotherapeutic Compass 2000/2001. In: Board] CvzCHI, ed. Amstelveen; 2000.

172. Statline Consumer Pricing Index. 2009. (Accessed at www.statline.nl.)

173. Vijgen SM, Koopmans CM, Opmeer BC, et al. An economic analysis of induction of labour and expectant monitoring in women with gestational hypertension or preeclampsia at term (HYPITAT trial). Bjog 2010;117:1577-85.

174. Teune MJ, Bakhuizen S, Gyamfi Bannerman C, et al. A systematic review of severe morbidity in infants born late preterm. Am J Obstet Gynecol 2011;205:374 e1-9.

175. Boyle EM, Poulsen G, Field DJ, et al. Effects of gestational age at birth on health outcomes at 3 and 5 years of age: population based cohort study. Bmj 2012;344:e896.

176. Vis JY, Wilms FF, Oudijk MA, et al. Why were the results of randomized trials on the clinical utility of fetal fibronectin negative? A systematic review of their study designs. Am J Perinatol 2011;28:145-50.

177. Sanchez-Ramos L, Delke I, Zamora J, Kaunitz AM. Fetal fibronectin as a short-term predictor of preterm birth in symptomatic patients: a meta-analysis. Obstet Gynecol 2009;114:631-40.

178. Honest H, Bachmann LM, Gupta JK, Kleijnen J, Khan KS. Accuracy of cervicovaginal fetal fibronectin test in predicting risk of spontaneous preterm birth: systematic review. Bmj 2002;325:301.

179. Wilms FF, van Stralen G, Porath MM, et al. [Predicating imminent preterm labour based on a determination of foetal fibronectin in a vaginal smear]. Ned Tijdschr Geneeskd 2009;153:B398.

180. Mol BW, Ruifrok AE. Global Alignment, Coordination and Collaboration in Perinatal Research: The Global Obstetrics Network (GONet) Initiative. Am J Perinatol 2012.

181. Edwards MS, Baker CJ. Bacterial infections in the neonate. In: Long SS, Pickering LK, Prober CG, eds. Principles and Practice of Pediatric Infectious Disease. 4th ed. Philadelphia: Elsevier Saunders; 2012.

182. Baker CJ, Barrett FF. Transmission of group B streptococci among parturient women and their neonates. J Pediatr 1973;83:919-25.

183. Schrag SJ, Zywicki S, Farley MM, et al. Group B streptococcal disease in the era of intrapartum antibiotic prophylaxis. New Eng J Med 2000;342:15-20.

184. Schrag S, Gorwitz R, Fultz-Butts K, Schuchat A. Prevention of perinatal group B streptococcal disease. Revised guidelines from CDC. MMWR Recomm Rep 2002;51:1-22.

185. Herbst A, Kallen K. Time between membrane rupture and delivery and septicemia in term neonates. Obstet Gynecol 2007;110:612-8.

186. Schucker JL, Mercer BM. Midtrimester premature rupture of the membranes. Seminars in perinatology 1996;20:389-400.

187. Ernest JM. Neonatal consequences of preterm PROM. Clinical obstetrics and gynecology 1998;41:827-31. 
188. Assessment of Amnioninfusion for improving perinatal outcomes after midtrimester preterm prelabour rupture of membranes. Nederlands Trial Register, 2012. http:// www.trialregister.nl/trialreg/admin/rctview.asp?TC=3492.

189. Van der Ham DP, Mol BWJ, Schaaf JM, Kazemier BM, Ravelli AC. Pregnancy outcome of subsequent pregnancy after a history of preterm birth with and without PPROM. In: 33rd Annual Meeting of the Society of Maternal Fetal Medicine. San Francisco; 2013

190. Lim AC, Schuit E, Bloemenkamp K, et al. 17alpha-hydroxyprogesterone caproate for the prevention of adverse neonatal outcome in multiple pregnancies: a randomized controlled trial. Obstet and Gynecol 2011;118:513-20.

191. Roos C, Spaanderman ME, Schuit E, et al. Effect of maintenance tocolysis with nifedipine in threatened preterm labor on perinatal outcomes: a randomized controlled trial. Jama 2013;309:41-7.

192. Bakker JJ, Verhoeven CJ, Janssen PF, et al. Outcomes after internal versus external tocodynamometry for monitoring labor. N Eng J Med 2010;362:306-13.

193. Jozwiak M, Oude Rengerink K, Benthem M, et al. Foley catheter versus vaginal prostaglandin E2 gel for induction of labour at term (PROBAAT trial): an open-label, randomised controlled trial. Lancet 2011;378:2095-103.

194. Westerhuis ME, Visser GH, Moons KG, Zuithoff N, Mol BW, Kwee A. Cardiotocography plus ST analysis of fetal electrocardiogram compared with cardiotocography only for intrapartum monitoring: a randomized controlled trial. Obstet and Gynecol 2011;117:406-7.

195. Kaandorp SP, Goddijn M, van der Post JA, et al. Aspirin plus heparin or aspirin alone in women with recurrent miscarriage. N Eng J Med 2010;362:1586-96.

196. Custers IM, Flierman PA, Maas P, et al. Immobilisation versus immediate mobilisation after intrauterine insemination: randomised controlled trial. Bmj 2009;339:b4080.

197. Mourits MJ, Bijen CB, Arts HJ, et al. Safety of laparoscopy versus laparotomy in earlystage endometrial cancer: a randomised trial. Lancet Oncol 2010;11:763-71.

198. van Santvoort HC, Besselink MG, Gooszen HG. [Obtaining medical ethical approval for a multicentre, randomised study: prospective evaluation of a ponderous process]. Ned Tijdschr Geneeskd 2008;152:2077-83.

199. van der Tuuk K, Koopmans CM, Groen H, Mol BW, van Pampus MG. Impact of the HYPITAT trial on doctors' behaviour and prevalence of eclampsia in the Netherlands. Bjog 2011;118:1658-60.

\section{REFERENCE LIST IN ALPHABETICAL ORDER}

Abdelazim, I. A. and H. H. Makhlouf (2012). "Placental alpha microglobulin-1 (AmniSure((R)) test) for detection of premature rupture of fetal membranes." Archives of Gynecology and Obstetrics 285(4): 985-989.

ACOG (2007). "ACOG Practice Bulletin No. 80: premature rupture of membranes. Clinical management guidelines for obstetrician-gynecologists." Obstet Gynecol 109(4): 1007-1019.

Albayrak, M., I. Ozdemir, et al. (2011). "Comparison of the diagnostic efficacy of the two rapid bedside immunoassays and combined clinical conventional diagnosis in prelabour rupture of membranes." Eur J Obst Gynecol Reprod Biol 158(2): 179-182.

Alexander, J. M., D. M. Mclntire, et al. (1999). "Chorioamnionitis and the prognosis for term infants." Obstet Gynecol 94(2): 274-278.

Allison, P. (2001). Missing Data. Iowa, Sage.

Anai, T., Y. Tanaka, et al. (1997). "Vaginal fluid hCG levels for detecting premature rupture of membranes." Obstet Gynecol 89(2): 261-264.

Arias, F. and P. Tomich (1982). "Etiology and outcome of low birth weight and preterm infants." Obstet and Gynecol 60(3): 277-281.

Averette, H. E., D. O. Hammond, et al. (1967). “Endocrine colpocytology and cytodiagno- 
sis of ruptured fetal membranes." Jama 202(7): 640-644.

Baker, C. J. and F. F. Barrett (1973). "Transmission of group B streptococci among parturient women and their neonates." J Pediatrics 83(6): 919-925.

Bakker, J. J., C. J. Verhoeven, et al. (2010). "Outcomes after internal versus external tocodynamometry for monitoring labor." N Eng J Med 362(4): 306-313.

Bank, C. M., J. P. Offermans, et al. (1991). "Diamine oxidase activity in amniotic fluid for diagnosis of ruptured membranes." Eur J Clin Chem Clin Biochem 29(11): 743-748.

Bankowska, E. M., J. Leibschang, et al. (2003). "[Usefulness of determination of granulocyte elastase plasma level, c-reactive protein and white blood cell count in prediction in intrauterine infection in pregnant women after PROM]." Ginekol Pol 74(10): 1037-1043.

Baptisti, A. (1938). "Chemical test for the determination of ruptured membranes." Am J Obstet Gynecol 87: 688-690.

Beckmann, M. W., I. Wiegratz, et al. (1993). "[Diagnosis of rupture of fetal membranes: comparison of vaginal detection of fetal fibronectin and intra-amnion injection of indigo carmine]." Geburtshilfe Frauenheilkd 53(2): 86-91.

Bell, M. J., J. L. Ternberg, et al. (1978). "Neonatal necrotizing enterocolitis. Therapeutic decisions based upon clinical staging." Ann Surgery 187(1): 1-7.

Berardi, J. C., S. Hutin, et al. (1991). "[The value of C-reactive protein in the detection of chorioamnionitis in cases of premature rupture of membranes]." Rev Fr Gynecol Obstet 86(3): 229-232.

Birkenmaier, A., J. J. Ries, et al. (2011). "Placental alpha-microglobulin-1 to detect uncertain rupture of membranes in a European cohort of pregnancies." Archives of Gynecology and Obstetrics.

Boers, K. E., S. M. Vijgen, et al. (2010). "Induction versus expectant monitoring for intrauterine growth restriction at term: randomised equivalence trial (DIGITAT)." Bmj 341: c7087.

Bornstein, J., A. Geva, et al. (2006). "Nonintrusive diagnosis of premature ruptured amniotic membranes using a novel polymer." Am J Perinatol 23(6): 351-354.

Bornstein, J., G. Ohel, et al. (2009). "Effectiveness of a novel home-based testing device for the detection of rupture of membranes." Am J Perinatol 26(1): 45-50.

Bossuyt, P. M., J. B. Reitsma, et al. (2003). "Towards complete and accurate reporting of studies of diagnostic accuracy: the STARD initiative." Bmj 326(7379): 41-44.

Boyle, E. M., G. Poulsen, et al. (2012). "Effects of gestational age at birth on health outcomes at 3 and 5 years of age: population based cohort study." Bmj 344: e896.

Buchanan, S., C. Crowther, et al. (2004). "Preterm prelabour rupture of the membranes: a survey of current practice." Aust N Z J Obstet Gynaecol 44(5): 400-403.

Buchanan, S. L., C. A. Crowther, et al. (2010). "Planned early birth versus expectant management for women with preterm prelabour rupture of membranes prior to 37 weeks' gestation for improving pregnancy outcome." Cochrane database of systematic reviews(3): CD004735.

Burns, C. M., M. A. Rutherford, et al. (2008). "Patterns of cerebral injury and neurodevelopmental outcomes after symptomatic neonatal hypoglycemia." Pediatrics 122(1): 65-74.

Buyukbayrak, E. E., C. Turan, et al. (2004). "Diagnostic power of the vaginal washing-fluid prolactin assay as an alternative method for the diagnosis of premature rupture of membranes." J Matern Fetal Neonatal Med 15(2): 120-125.

Cooper, S., I. Lange, et al. (2012). "Diagnostic accuracy of rapid phIGFBP-I assay for predicting preterm labor in symptomatic patients." J Perinatol 32(6): 460-465.

Cousins, L. M., D. P. Smok, et al. (2005). "AmniSure placental alpha microglobulin-1 rapid immunoassay versus standard diagnostic methods for detection of rupture of membranes." Am J Perinatol 22(6): 317-320.

Cowett, R. M., D. O. Hakanson, et al. (1976). "Untoward neonatal effect of intraamniotic administration of methylene blue." Obstet Gynecol 48(1 Suppl): 74S-75S.

Cox, S. M. and K. J. Leveno (1995). "Intentional delivery versus expectant management 
with preterm ruptured membranes at 30-34 weeks' gestation." Obstet Gynecol 86(6): 875-879.

Cox, S. M., M. L. Williams, et al. (1988). "The natural history of preterm ruptured membranes: what to expect of expectant management." Obstet Gynecol 71(4): 558-562.

Custers, I. M., P. A. Flierman, et al. (2009). "Immobilisation versus immediate mobilisation after intrauterine insemination: randomised controlled trial." Bmj 339: b4080.

de Haan, H. H., P. M. Offermans, et al. (1994). "Value of the fern test to confirm or reject the diagnosis of ruptured membranes is modest in nonlaboring women presenting with nonspecific vaginal fluid loss." Am J Perinatol 11(1): 46-50.

de Vries, L. S., P. Eken, et al. (1992). "The spectrum of leukomalacia using cranial ultrasound." Behavioural brain research 49(1): 1-6.

Deeks, J. J. (2001). "Systematic reviews in health care: Systematic reviews of evaluations of diagnostic and screening tests." Bmj 323(7305): 157-162.

Deville, W. L., F. Buntinx, et al. (2002). "Conducting systematic reviews of diagnostic studies: didactic guidelines." BMC Med Res Methodol 2: 9.

Di Renzo, G. C., L. C. Roura, et al. (2011). "Guidelines for the management of spontaneous preterm labor: identification of spontaneous preterm labor, diagnosis of preterm premature rupture of membranes, and preventive tools for preterm birth." The journal of maternal-fetal \& neonatal medicine : J Matern Fetal Neonal 2011;24:659-67.

Dodds, W. G. and J. D. lams (1987). "Maternal C-reactive protein and preterm labor." J Reprod Med 32(7): 527-530.

Donders, A. R., G. J. van der Heijden, et al. (2006). "Review: a gentle introduction to imputation of missing values." J Clin Epidemiol 59(10): 1087-1091.

Drummond, M. F., M. J. Sculpher, et al. (2005). Methods for the Economic Evaluation of Health Care Programmes. Oxford, Oxford University Press.

Edwards, M. S. and C. J. Baker (2012). Bacterial infections in the neonate. Principles and Practice of Pediatric Infectious Disease. S. S. Long, L. K. Pickering and C. G. Prober. Philadelphia, Elsevier Saunders.

Elmfors, B., N. Tryding, et al. (1974). "The diagnosis of ruptured fetal membranes by measurement of the diamine oxidase (DAO) activity in vaginal fluid." J Obstet Gynaecol Br Commonw 81(5): 361-362.

Erdemoglu, E. and T. Mungan (2004). "Significance of detecting insulin-like growth factor binding protein-1 in cervicovaginal secretions: comparison with nitrazine test and amniotic fluid volume assessment." Acta Obstet Gynecol Scand 83(7): 622-626.

Eriksen, N. L., V. M. Parisi, et al. (1992). "Fetal fibronectin: a method for detecting the presence of amniotic fluid." Obstet Gynecol 80(3 Pt 1): 451-454.

Ernest, J. M. (1998). "Neonatal consequences of preterm PROM." Clinical obstetrics and gynecology 41(4): 827-831.

Evans, M. I., S. N. Hajj, et al. (1980). "C-reactive protein as a predictor of infectious morbidity with premature rupture of membranes." Am J Obstet Gynecol 138(6): 648652.

Farb, H. F., M. Arnesen, et al. (1983). "C-reactive protein with premature rupture of membranes and premature labor." Obstet Gynecol 62(1): 49-51.

Fisk, N. M., J. Fysh, et al. (1987). "Is C-reactive protein really useful in preterm premature rupture of the membranes?" Bjog 94(12): 1159-1164.

Friedman, M. L. and T. W. McElin (1969). "Diagnosis of ruptured fetal membranes. Clinical study and review of the literature." Am J Obstet Gynecol 104(4): 544-550.

Fujimoto, S., T. Kishida, et al. (1995). "Clinical usefulness of the dye-injection method for diagnosing premature rupture of the membranes in equivocal cases." J Obstet Gynaecol 21(3): 215-220.

Furman, B., I. Shoham-Vardi, et al. (2000). "Clinical significance and outcome of preterm prelabor rupture of membranes: population-based study." Eur J Obstet Gynecol Reprod Biol 92(2): 209-216.

Gariepy, G. and R. Vauclair (1969). "Study of ruptured fetal membranes by Nile blue sulfate staining." Acta Cytol 13(3): 154-157. 
Garite, T. J. (2001). "Management of premature rupture of membranes." Clinics in perinatology 28(4): 837-847.

Garite, T. J., R. K. Freeman, et al. (1981). "Prospective randomized study of corticosteroids in the management of premature rupture of the membranes and the premature gestation." Am J Obstet Gynecol 141(5): 508-515.

Gaucherand, P., B. Salle, et al. (1997). "Comparative study of three vaginal markers of the premature rupture of membranes. Insulin like growth factor binding protein 1 diamine-oxidase pH." Acta Obstet Gynecol Scand 76(6): 536-540.

Gibbs, R. S. and J. D. Blanco (1982). "Premature rupture of the membranes." Obstet Gynecol 60(6): 671-679.

Goldenberg, R. L. (2002). "The management of preterm labor." Obstet Gynecol 100(5 Pt 1): $1020-1037$.

Goldenberg, R. L., K. G. Nelson, et al. (1984). "Delay in delivery: influence of gestational age and the duration of delay on perinatal outcome." Obstet Gynecol 64(4): 480484.

Goldstein, B., B. Giroir, et al. (2005). "International pediatric sepsis consensus conference: definitions for sepsis and organ dysfunction in pediatrics." Pediatr Crit Care Med 6(1): 2-8.

Gonen, R., M. E. Hannah, et al. (1989). "Does prolonged preterm premature rupture of the membranes predispose to abruptio placentae?" Obstet Gynecol 74(3 Pt 1): 347-350.

Gonik, B., C. Weiner, et al., Eds. (2005). High risk pregnancy management options. Philadelphia, W.B. Saunders Company.

Gorodeski, I. G., L. Haimovitz, et al. (1982). "Reevaluation of the pH, ferning and nile blue sulphate staining methods in pregnant women with premature rupture of the fetal membranes." J Perinat Med 10(6): 286-292.

Gorodeski, I. G., M. Paz, et al. (1979). "Diagnosis of rupture of the fetal membranes by glucose and fructose measurements." Obstet Gynecol 53(5): 611-616.

Gunn, G. C., D. R. Mishell, Jr., et al. (1970). "Premature rupture of the fetal membranes. A review." Am J Obstet Gynecol 106(3): 469-483.

Gurbuz, A., A. Karateke, et al. (2004). "Vaginal fluid creatinine in premature rupture of membranes." Int J Gynaecol Obstet 85(3): 270-271.

Hanley, J. A. and B. J. McNeil (1983). "A method of comparing the areas under receiver operating characteristic curves derived from the same cases." Radiology 148(3): 839-843.

Hannah, M. E., A. Ohlsson, et al. (1996). "Induction of labor compared with expectant management for prelabor rupture of the membranes at term. TERMPROM Study Group." N Engl J Med 334(16): 1005-1010.

Hansen, A. K., K. Wisborg, et al. (2008). "Risk of respiratory morbidity in term infants delivered by elective caesarean section: cohort study." Bmj 336(7635): 85-87.

Harrell, F. J. (2001). Regression Modeling Strategies. New York, Springer.

Hartling, L., R. Chari, et al. (2006). "A systematic review of intentional delivery in women with preterm prelabor rupture of membranes." J Matern Fetal Neonatal Med 19(3): 177-187.

Hawrylyshyn, P., P. Bernstein, et al. (1983). "Premature rupture of membranes: the role of C-reactive protein in the prediction of chorioamnionitis." Am J Obstet Gynecol 147(3): 240-246.

Hays, W. (1998). Statistics. New York, Holt, Rinehart and Winston.

He, J. P. (1990). "[C-reactive protein and acute-phase proteins in monitoring infection caused by premature rupture of membranes]." Zhonghua Fu Chan Ke Za Zhi 25(2): 98-101, 125.

Hellemans, P., P. Verdonk, et al. (1992). "Preliminary results with the use of the ROMcheck immunoassay in the early detection of rupture of the amniotic membranes." Eur J Obstet Gynecol Reprod Biol 43(3): 173-179.

Herbst, A. and K. Kallen (2007). "Time between membrane rupture and delivery and 
septicemia in term neonates." Obstet Gynecol 110(3): 612-618.

Honest, H., L. M. Bachmann, et al. (2002). "Accuracy of cervicovaginal fetal fibronectin test in predicting risk of spontaneous preterm birth: systematic review." Bmj 325(7359): 301.

lams, J. D., M. L. Talbert, et al. (1985). "Management of preterm prematurely ruptured membranes: a prospective randomized comparison of observation versus use of steroids and timed delivery." Am J Obstet Gynecol 151(1): 32-38.

Ibarra Chavarria, V., P. Sanhueza Smith, et al. (1989). "[C-reactive protein as early marker of chorioamnionitis in premature rupture of membranes]." Ginecol Obstet Mex 57: 203-208.

Ismail, M. A., M. J. Zinaman, et al. (1985). "The significance of C-reactive protein levels in women with premature rupture of membranes." Am J Obstet Gynecol 151(4): 541-544.

Jeurgens-Borst, A. J., R. L. Bekkers, et al. (2002). "Use of insulin like growth factor binding protein-1 in the diagnosis of ruptured fetal membranes." Eur J Obstet Gynecol Reprod Biol 102(1): 11-14.

Jozwiak, M., K. Oude Rengerink, et al. (2011). "Foley catheter versus vaginal prostaglandin E2 gel for induction of labour at term (PROBAAT trial): an open-label, randomised controlled trial." Lancet 378(9809): 2095-2103.

Kaandorp, S. P., M. Goddijn, et al. (2010). "Aspirin plus heparin or aspirin alone in women with recurrent miscarriage." New Eng J Med 362(17): 1586-1596.

Kafali, H. and C. Oksuzler (2007). "Vaginal fluid urea and creatinine in diagnosis of premature rupture of membranes." Archives of Gynecology and Obstetrics 275(3): 157160.

Kayem, G., A. Bernier-Dupreelle, et al. (2010). "Active versus expectant management for preterm prelabor rupture of membranes at 34-36 weeks of completed gestation: comparison of maternal and neonatal outcomes." Acta Obstet Gynecol Scand 89(6): 776-781.

Kim, Y. H., Y. W. Park, et al. (2005). "Vaginal fluid beta-human chorionic gonadotropin level in the diagnosis of premature rupture of membranes." Acta Obstet Gynecol Scand 84(8): 802-805.

Koninckx, P. R., H. Trappeniers, et al. (1981). "Prolactin concentration in vaginal fluid: a new method for diagnosing ruptured membranes." Bjog 88(6): 607-610.

Koopmans, C. M., D. Bijlenga, et al. (2009). "Induction of labour versus expectant monitoring for gestational hypertension or mild pre-eclampsia after 36 weeks' gestation (HYPITAT): a multicentre, open-label randomised controlled trial." Lancet 374(9694): 979-988.

Kubota, T. and H. Takeuchi (1998). "Evaluation of insulin-like growth factor binding protein-1 as a diagnostic tool for rupture of the membranes." J Obstet Gynaecol Res 24(6): 411-417.

Kurki, T., K. Teramo, et al. (1990). "C-reactive protein in preterm premature rupture of the membranes." Arch Gynecol Obstet 247(1): 31-37.

Ladfors, L., L. A. Mattsson, et al. (1997). "Is a speculum examination sufficient for excluding the diagnosis of ruptured fetal membranes?" Acta Obstet Gynecol Scand 76(8): 739-742.

Langenveld, J., A. C. Ravelli, et al. (2011). "Neonatal outcome of pregnancies complicated by hypertensive disorders between 34 and 37 weeks of gestation: a 7 year retrospective analysis of a national registry." Am J Obstet Gynecol. Dec;205(6):540.e1-7

Lau, J., F. Magee, et al. (2005). "Chorioamnionitis with a fetal inflammatory response is associated with higher neonatal mortality, morbidity, and resource use than chorioamnionitis displaying a maternal inflammatory response only." Am J Obstet Gynecol. 193(3 Pt 1): 708-713.

Lee, J., S. M. Lee, et al. (2011). "Fragmented forms of insulin-like growth factor binding protein-1 in amniotic fluid of patients with preterm labor and intact membranes." Reproductive sciences 18(9): 842-849. 
Lee, S. E., J. S. Park, et al. (2007). "Measurement of placental alpha-microglobulin-1 in cervicovaginal discharge to diagnose rupture of membranes." Obstet Gynecol 109(3): 634-640.

Lee, S. M., J. Lee, et al. (2009). "The clinical significance of a positive Amnisure test in women with term labor with intact membranes." J Matern Fetal Neonal 22(4): 305310.

Leviton, A., E. N. Allred, et al. (2010). "Microbiologic and histologic characteristics of the extremely preterm infant's placenta predict white matter damage and later cerebral palsy. the ELGAN study." Pediatric research 67(1): 95-101.

Lewis, D. F., S. Futayyeh, et al. (1996). "Preterm delivery from 34 to 37 weeks of gestation: is respiratory distress syndrome a problem?" Am J Obstet Gynecol 174(2): 525-528.

Lieman, J. M., C. G. Brumfield, et al. (2005). "Preterm premature rupture of membranes: is there an optimal gestational age for delivery?" Obstet Gynecol 105(1): 12-17.

Lim, A. C., E. Schuit, et al. (2011). "17alpha-hydroxyprogesterone caproate for the prevention of adverse neonatal outcome in multiple pregnancies: a randomized controlled trial." Obstet Gynecol 118(3): 513-520.

Lucas, A., R. Morley, et al. (1988). "Adverse neurodevelopmental outcome of moderate neonatal hypoglycaemia." Bmj 297(6659): 1304-1308.

Mackay, D. F., G. C. Smith, et al. (2010). "Gestational age at delivery and special educational need: retrospective cohort study of 407,503 schoolchildren." PLoS Medicine 7(6): e1000289.

Major, C. A., M. de Veciana, et al. (1995). "Preterm premature rupture of membranes and abruptio placentae: is there an association between these pregnancy complications?" Am J Obstet Gynecol 172(2 Pt 1): 672-676.

Mateus, J., K. Fox, et al. (2010). "Preterm premature rupture of membranes: clinical outcomes of late-preterm infants." Clinical pediatrics 49(1): 60-65.

McEnerney, J. K. and L. N. McEnerney (1983). "Unfavorable neonatal outcome after intraamniotic injection of methylene blue." Obstet Gynecol 61(3 Suppl): 35S-37S.

Mead, P. B. (1980). "Management of the patient with premature rupture of the membranes." Clin Perinatol 7(2): 243-255.

Meray, N., J. B. Reitsma, et al. (2007). "Probabilistic record linkage is a valid and transparent tool to combine databases without a patient identification number." J Clin Epidemiol 60(9): 883-891.

Mercer, B. M. (2003). "Preterm premature rupture of the membranes." Obstet Gynecol 101(1): 178-193.

Mercer, B. M., L. G. Crocker, et al. (1993). "Induction versus expectant management in premature rupture of the membranes with mature amniotic fluid at 32 to 36 weeks: a randomized trial." Am J Obstet Gynecol 169(4): 775-782.

Mercer, B. M., R. L. Goldenberg, et al. (2000). "The Preterm Prediction Study: prediction of preterm premature rupture of membranes through clinical findings and ancillary testing. The National Institute of Child Health and Human Development MaternalFetal Medicine Units Network." Am J Obstet Gynecol 183(3): 738-745.

Merenstein, G. B. and L. E. Weisman (1996). "Premature rupture of the membranes: neonatal consequences." Semin Perinatol 20(5): 375-380.

Meyer, B. A., B. Gonik, et al. (1991). "Evaluation of phenazopyridine hydrochloride as a tool in the diagnosis of premature rupture of the membranes." Am J Perinatol 8(5): 297-299.

Mol, B. W. and A. E. Ruifrok (2012). "Global Alignment, Coordination and Collaboration in Perinatal Research: The Global Obstetrics Network (GONet) Initiative." Am J Perinatol

Morris, J. and C. Crowther (2005. ). A randomised controlled trial of immediate delivery versus expectant care in women with ruptured membranes close to term. ISRCTN 44485060. Australian National Health and Medical Research Council. , Royal North Shore Hospital, Australia,.

Morris, J. M., C. L. Roberts, et al. (2006). "Protocol for the immediate delivery versus ex- 
pectant care of women with preterm prelabour rupture of the membranes close to term (PPROMT) Trial [ISRCTN44485060]." BMC Pregnancy Childbirth 6: 9.

Mourits, M. J., C. B. Bijen, et al. (2010). "Safety of laparoscopy versus laparotomy in earlystage endometrial cancer: a randomised trial." Lancet Oncol 11(8): 763-771.

Mulhair, L., J. Carter, et al. (2009). "Prospective cohort study investigating the reliability of the AmnioSense(trademark) method for detection of spontaneous rupture of membranes." Bjog 116(2): 313-318.

Naef, R. W., 3rd, J. R. Allbert, et al. (1998). "Premature rupture of membranes at 34 to 37 weeks' gestation: aggressive versus conservative management." Am J Obstet Gynecol 178(1 Pt 1): 126-130.

Neerhof, M. G., C. Cravello, et al. (1999). "Timing of labor induction after premature rupture of membranes between 32 and 36 weeks' gestation." Am J Obstet Gynecol 180(2 Pt 1): 349-352.

Neil, P. R. and E. M. Wallace (2010). "Is Amnisure(R) useful in the management of women with prelabour rupture of the membranes?" Aus N Z J Obstet Gynaecol 50(6): 534538.

Nelson, L. H., P. J. Meis, et al. (1985). "Premature rupture of membranes: a prospective, randomized evaluation of steroids, latent phase, and expectant management." $\mathrm{Ob}$ stet Gynecol 66(1): 55-58.

Netherlands, S. (2009). "Statline Consumer Pricing Index." from www.statline.nl.

Neu, J. (1996). "Necrotizing enterocolitis: the search for a unifying pathogenic theory leading to prevention." Pediatr Clin North Am 43(2): 409-432.

Newman, T. B., P. Liljestrand, et al. (2006). "Outcomes among newborns with total serum bilirubin levels of 25 mg per deciliter or more." N Engl J Med 354(18): 1889-1900.

$\mathrm{Ni}$, C. Y., W. X. Jia, et al. (2003). "Practicability of using vaginal fluid markers in detecting premature rupture of membranes." Ann Clin Biochem 40(Pt 5): 542-545.

Nowak, M., P. Oszukowski, et al. (1998). "[Intrauterine infections. I. The role of C-reactive protein, white blood cell count and erythrocyte sedimentation rate in pregnant women in the detection of intrauterine infection after preliminary rupture of membranes]." Ginekol Pol 69(8): 615-622.

O'Shea, T. M., E. N. Allred, et al. (2009). "The ELGAN study of the brain and related disorders in extremely low gestational age newborns." Early human development 85(11): 719-725.

Oostenbrink, J. B. (2009). "Standardisation of costs: the Dutch Manual for Costing in Economic Evaluations." Pharmacoeconomics 20(7): 443-454.

Ottervanger, H. P., M. J. Keirse, et al. (1996). "Controlled comparison of induction versus expectant care for prelabor rupture of the membranes at term." J Perinat Med 24(3): 237-242.

Papile, L. A., J. Burstein, et al. (1978). "Incidence and evolution of subependymal and intraventricular hemorrhage: a study of infants with birth weights less than 1,500 gm." J Pediatrics 92(4): 529-534.

Pollet-Villard, M., R. Cartier, et al. (2011). "Detection of placental alpha microglobulin-1 versus insulin-like growth factor-binding protein-1 in amniotic fluid at term: a comparative study." Am J Perinatol 28(6): 489-494.

Ramsey, P. S., J. M. Lieman, et al. (2005). "Chorioamnionitis increases neonatal morbidity in pregnancies complicated by preterm premature rupture of membranes." $\mathrm{Am} \mathrm{J}$ Obstet Gynecol 192(4): 1162-1166.

Reitsma, J. B., A. S. Glas, et al. (2005). "Bivariate analysis of sensitivity and specificity produces informative summary measures in diagnostic reviews." J Clin Epidemiol 58(10): 982-990.

Riboni, F., A. Vitulo, et al. (2012). "Combination of biochemical markers in predicting preterm delivery." Archives of Gynecology and Obstetrics 285(1): 61-66.

Rochelson, B. L., D. A. Richardson, et al. (1983). "Rapid assay - possible application in the diagnosis of premature rupture of the membranes." Obstet Gynecol 62(4): 414-418.

Romem, Y. and R. Artal (1984). "C-reactive protein as a predictor for chorioamnionitis in 
cases of premature rupture of the membranes." Am J Obstet Gynecol 150(5 Pt 1): 546-550.

Roos, C., M. E. Spaanderman, et al. (2013). "Effect of maintenance tocolysis with nifedipine in threatened preterm labor on perinatal outcomes: a randomized controlled trial." Jama 309(1): 41-47.

Rubin, D. (1976). "Inference and missing data." Blometrika 63(3): 581-592.

Rutanen, E. M., T. H. Karkkainen, et al. (1996). "Evaluation of a rapid strip test for insulinlike growth factor binding protein-1 in the diagnosis of ruptured fetal membranes." Clinica chimica acta; 253(1-2): 91-101.

Rutjes, A. W., J. B. Reitsma, et al. (2007). "Evaluation of diagnostic tests when there is no gold standard. A review of methods." Health Technol Assess 11(50): iii, ix-51.

Sanchez-Ramos, L., I. Delke, et al. (2009). "Fetal fibronectin as a short-term predictor of preterm birth in symptomatic patients: a meta-analysis." Obstet Gynecol 114(3): 631-640.

Sarnat, H. B. and M. S. Sarnat (1976). "Neonatal encephalopathy following fetal distress. A clinical and electroencephalographic study." Arch Neurol 33(10): 696-705.

Schrag, S., R. Gorwitz, et al. (2002). "Prevention of perinatal group B streptococcal disease. Revised guidelines from CDC." MMWR. Recommendations and reports : Morbidity and mortality weekly report. Recommendations and reports / Centers for Disease Control 51(RR-11): 1-22.

Schrag, S. J., S. Zywicki, et al. (2000). "Group B streptococcal disease in the era of intrapartum antibiotic prophylaxis." New Eng J Med 342(1): 15-20.

Schucker, J. L. and B. M. Mercer (1996). "Midtrimester premature rupture of the membranes." Seminars in perinatology 20(5): 389-400.

Sereepapong, W., S. Limpongsanurak, et al. (2001). "The role of maternal serum C-reactive protein and white blood cell count in the prediction of chorioamnionitis in women with premature rupture of membranes." J Med Assoc Thai 84 Suppl 1: S360-366.

Sgro, M., P. S. Shah, et al. (2011). "Early-onset neonatal sepsis: rate and organism pattern between 2003 and 2008." J Perinatol 31(12): 794-798.

Shapiro, S. M. (2003). "Bilirubin toxicity in the developing nervous system." Pediatr Neurol 29(5): 410-421.

Silva, E. and J. Martinez (2009). "Diagnosing ROM: a comparison of the gold standard, indigo carmine amnioinfusion, to the rapid immunoassay, the AmniSure ROM test." J Perinat Med 37: 956.

Simhan, H. N. and T. P. Canavan (2005). "Preterm premature rupture of membranes: diagnosis, evaluation and management strategies." Bjog 112 Suppl 1: 32-37.

Smith, G., C. Rafuse, et al. (2005). "Prevalence, management, and outcomes of preterm prelabour rupture of the membranes of women in Canada." J Obstet Gynaecol Can 27(6): 547-553.

Smith, R. P. (1976). "A technic for the detection of rupture of the membranes. A review and preliminary report." Obstet Gynecol 48(2): 172-176.

Spinnato, J. A., D. C. Shaver, et al. (1987). "Preterm premature rupture of the membranes with fetal pulmonary maturity present: a prospective study." Obstet Gynecol 69(2): 196-201.

Steyerberg, E. W. (2009). Clinical Prediction Models. New York, Springer.

Steyerberg, E. W., A. J. Vickers, et al. (2010). "Assessing the performance of prediction models: a framework for traditional and novel measures." Epidemiology 21(1): 128138.

Sun, G. W., T. L. Shook, et al. (1996). "Inappropriate use of bivariable analysis to screen risk factors for use in multivariable analysis." J Clinical Epidemiol 49(8): 907-916.

Tagore, S. and K. Kwek (2010). "Comparative analysis of insulin-like growth factor binding protein-1 (IGFBP-1), placental alpha-microglobulin-1 (PAMG-1) and nitrazine test to diagnose premature rupture of membranes in pregnancy." J Perinat Med 38(6): 609-612.

Teune, M. J., S. Bakhuizen, et al. (2011). "A systematic review of severe morbidity in in- 
fants born late preterm." Am J Obstet Gynecol 205(4): 374 e371-379.

Tiufekchieva, E. (1985). "[C-reactive protein as an index of amniotic infection in premature rupture of the amniotic sac]." Akush Ginekol (Sofiia) 24(6): 35-41.

Trochez-Martinez, R. D., P. Smith, et al. (2007). "Use of C-reactive protein as a predictor of chorioamnionitis in preterm prelabour rupture of membranes: a systematic review." Bjog 114(7): 796-801.

Tromp, M., A. C. Ravelli, et al. (2008). "An efficient validation method of probabilistic record linkage including readmissions and twins." Methods of information in medicine 47(4): 356-363.

van de Laar, R., D. P. van der Ham, et al. (2009). "Accuracy of C-reactive protein determination in predicting chorioamnionitis and neonatal infection in pregnant women with premature rupture of membranes: a systematic review." Eur J Obstet Gynecol Reprod Biol 147(2): 124-129.

van der Ham, D. P., B. W. J. Mol, et al. (2013). Pregnancy outcome of subsequent pregnancy after a history of preterm birth with and without PPROM. 33rd Annual Meeting of the Society of Maternal Fetal Medicine. San Francisco.

van der Ham, D. P., J. G. Nijhuis, et al. (2007). "Induction of labour versus expectant management in women with preterm prelabour rupture of membranes between 34 and 37 weeks (the PPROMEXIL-trial)." BMC Pregnancy Childbirth 7: 11.

van der Ham, D. P., J. L. van der Heyden, et al. (2012). "Management of late-preterm premature rupture of membranes: the PPROMEXIL-2 trial." Am J Obstet Gynecol 207(4):276.e1-276.e10

van der Ham, D. P., M. J. van Melick, et al. (2011). "Methods for the diagnosis of rupture of the fetal membranes in equivocal cases: a systematic review." Eur J Obst Gynecol Reprod Biol 157(2): 123-127.

van der Ham, D. P., S. M. Vijgen, et al. (2012). "Induction of labor versus expectant management in women with preterm prelabor rupture of membranes between 34 and 37 weeks: a randomized controlled trial." PLoS Medicine 9(4): e1001208.

van der Heyden, J. L., S. S. van Teeffelen, et al. (2010). "Is it useful to measure C-reactive protein and leukocytes in patients with prelabor rupture of membranes?" Am J Perinatol 27(7): 543-547.

van der Kuy, A. (2000). Pharmacotherapeutic Compass 2000/2001. C. v. z. C. H. I. Board]. Amstelveen.

van der Tuuk, K., C. M. Koopmans, et al. (2011). "Impact of the HYPITAT trial on doctors' behaviour and prevalence of eclampsia in the Netherlands." Bjog 118(13): 16581660.

van Houwelingen, H. C., L. R. Arends, et al. (2002). "Advanced methods in meta-analysis: multivariate approach and meta-regression." Stat Med 21(4): 589-624.

van Houwelingen, H. C., K. H. Zwinderman, et al. (1993). "A bivariate approach to metaanalysis." Stat Med 12(24): 2273-2284.

van Santvoort, H. C., M. G. Besselink, et al. (2008). "[Obtaining medical ethical approval for a multicentre, randomised study: prospective evaluation of a ponderous process]." NTVG 152(38): 2077-2083.

Vijgen, S. M., C. M. Koopmans, et al. (2010). "An economic analysis of induction of labour and expectant monitoring in women with gestational hypertension or pre-eclampsia at term (HYPITAT trial)." Bjog 117(13): 1577-1585.

Vis, J. Y., F. F. Wilms, et al. (2011). "Why were the results of randomized trials on the clinical utility of fetal fibronectin negative? A systematic review of their study designs." Am J Perinatol 28(2): 145-150.

Wang, T., R. Zhou, et al. (2011). "Proteins in leaked amniotic fluid as biomarkers diagnostic for prelabor rupture of membranes." Proteomics. Clinical applications 5(7-8): 415-421.

Watanabe, T., H. Minakami, et al. (1995). "Evaluation of latex agglutination test for alphafetoprotein in diagnosing rupture of fetal membranes." Gynecol Obstet Invest 39(1): 15-18. 
Westerhuis, M. E., G. H. Visser, et al. (2011). "Cardiotocography plus ST analysis of fetal electrocardiogram compared with cardiotocography only for intrapartum monitoring: a randomized controlled trial." Obstet Gynecol 117(2 Pt 1): 406-407.

Weston, E. J., T. Pondo, et al. (2011). "The burden of invasive early-onset neonatal sepsis in the United States, 2005-2008." The Pediatric infectious disease journal 30(11): 937-941.

Whiting, P., A. W. Rutjes, et al. (2003). "The development of QUADAS: a tool for the quality assessment of studies of diagnostic accuracy included in systematic reviews." BMC Med Res Methodol 3: 25.

Wiberg-Itzel, E., S. Cnattingius, et al. (2005). "Lactate determination in vaginal fluids: a new method in the diagnosis of prelabour rupture of membranes." Bjog 112(6): 754-758.

Wiberg-Itzel, E., H. Pettersson, et al. (2009). "Prediction of time to spontaneous onset of labour with lactate concentration in vaginal fluid in women with suspected preterm prelabour rupture of the membranes." Bjog 116(1): 62-66.

Williams, H. and H. Jeffery (1994). "The incidence of histological chorioamnionitis in IVF/ GIFT preterm births." Aust N Z J Obstet Gynaecol 34(4): 480-483.

Wilms, F. F., G. van Stralen, et al. (2009). "[Predicating imminent preterm labour based on a determination of foetal fibronectin in a vaginal smear]." NTVG 153: B398.

Yamada, H., T. Kishida, et al. (1997). "Comparison of an improved AFP kit with the intraamniotic PSP dye-injection method in equivocal cases of preterm premature rupture of the fetal membranes." J Obstet Gynaecol Res 23(3): 307-311.

Yoon, B. H., J. K. Jun, et al. (1996). "Serum C-reactive protein, white blood cell count, and amniotic fluid white blood cell count in women with preterm premature rupture of membranes." Obstet Gynecol 88(6): 1034-1040.

Zanjani, M. S. and L. Haghighi (2012). "Vaginal fluid creatinine for the detection of premature rupture of membranes." J Obst Gynaecol Research 38(3): 505-508.

\section{TRIAL REGISTRY}

Austrialian PROMT trial

"A randomised controlled trial of immediate delivery versus expectant care in women with ruptured membranes close to term." from http://www.controlled-trials. com/mrct/trial/228805/PPROMT.

Canadian Preterm PROM trial

"Clinical trial: Safety and Efficacy Study of Intentional Delivery in Women With Preterm and Prelabour Rupture of the Membranes." from http://clinicaltrials.gov/ct/ show/NCT00259519; jsessionid=511720C5DF99149F8A22D293DBDF071E? ord er=1.

PPROMEXIL trial

"Preterm Premature Rupture Of Membranes between 34 and 37 weeks: EXpecatant management versus Induction of Labour." from http://www.controlled-trials.com/ $\mathrm{mrct} /$ trial/257003/PPROMEXIL.

PPROMEXIL III trial

"Assessment of Amnioninfusion for improving perinatal outcomes after midtrimester preterm prelabour rupture of membranes." http://www.trialregister.nl/trialreg/ admin/rctview.asp?TC=3492.

ZonMW trialregistration PPROMEXIL trial

"ZonMw-Projectenpoort - Induction of labour versus expectant management in women with preterm premature rupture of membranes between 34 and 37 weeks." from http://zonmw.collexis.net/projectsummary.asp?foreignid=94507212 


\section{ONLINE GUIDELINES}

Dutch Society of Obstetrics and Gynaecology (NVOG)

(2002). "[Rupture of membranes before onset of labor]." http://nvog-documenten. $\mathrm{nl} /$ index.php?pagina=/richtlijn/pagina.php\&fSelectTG_62=75\&fSelectedSub=62\&fS electedParent $=75$.

"[Induction of labor]." NVOGnet voorlichting \& richtlijnen Retrieved 28 September, 2010, from http://novog-documenten.nl/index.php?pagina=/richtlijn/item/pagina. php\&richtlijn_id=689.

Royal Collage of Obstetrics and Gynaecology (RCOG)

(2002). Gynaecological Examinations: Guidelines for Specialist Practice. London, RCOG.

(2006). "Preterm prelabour rupture of membranes." http://www.rcog.org.uk/files/ rcog-corp/uploaded-files/GT44PretermPrelabourRupture2006.pdf.

\section{OTHERS}

PRN-foundation. "The Netherlands Perinatal Registry." from http://www.perinatreg. nl/home_english.

(2011). Review Manager (RevMan) Copenhagen, The Nordic Cochrane Centre, The Cochrane Collaboration. 


\section{LIST OF PUBLICATIONS}

THIS THESIS

DP van der Ham, ASP van Teeffelen, BWJ Mol. Prelabour Rupture of Membranes - overview of diagnostic methods. Curr Opin Obstet Gynecol. 2012 Dec;24(6):408-12

DP van der Ham, JL van der Heyden, BC Opmeer, ALM Mulder, RMJ Moonen, JJ van Beek, MTM Franssen, KWM Bloemenkamp, JM Sikkema, CJM de Groot, M Porath, A Kwee, MD Woiski, JJ Duvekot, BMC Akerboom, AJ van Loon, JW de Leeuw, C Willekes, BWJ Mol, JG Nijhuis. Management of late-preterm prelabor rupture of membranes: the PPROMEXIL-2 trial. Am J Obstet Gynecol. 2012 Oct;207(4):276.e1-10 - editor's choice.

DP van der Ham, SMC Vijgen, JG Nijhuis, JJ van Beek, BC Opmeer, ALM Mulder, RMJ Moonen, M Groenewout, MG van Pampus, GD Mantel, KWM Bloemenkamp, WJ van Wijngaarden, JM Sikkema, MC Haak, PJM Pernet, M Porath, JFM Molkenboer, S Kuppens, A Kwee, ME Kars, M Spaanderman, MJN Weinans, HIJ Wildschut, BMC Akerboom, BWJ Mol, C Willekes. Induction of labor versus Expectant Management in Women with Preterm Prelabor Rupture of Membranes between 34 and 37 weeks: a Randomized Controlled Trial. PLoS Med. 2012 Apr. 9 (4). E1001208. Epub 2012 Apr 24

DP van der Ham, MM van Melick, L Smits, JG Nijhuis, CP Weiner, JJ van Beek, BWJ Mol, $C$ Willekes. Methods for the diagnosis of rupture of the fetal membranes in equivocal cases: a systematic review. Eur J Obstet Gynecol Reprod Biol. 2011 Aug; 157(2):123-7. Epub 2011 Apr 8

$\mathrm{R}$ van de Laar, DP van der Ham, SG Oei, C Willekes, CP Weiner, BWJ Mol. Accuracy of $C$-reactive protein determination in predicting chorioamnionitis and neonatal infection in pregnant women with premature rupture of membranes: A systematic review. Eur J Obstet Gynecol Reprod Biol. 2009 Dec;147(2):124-9. Epub 2009 Oct 12

DP van der Ham; JG Nijhuis, BWJ Mol, JJ van Beek, BC Opmeer, D Bijlenga, M Groenewout, B Arabin, KWM Bloemenkamp, WJ van Wijngaarden, MGAJ Wouters, PJM Pernet, MM Porath, JFM Molkenboer, JB Derks, MM Kars, HCJ Scheepers, MJN Weinans, MD Woiski, HIJ Wildschut, C Willekes. Induction of labour versus expectant managment in women with preterm prelabour rupture of membranes between 34 and 37 weeks (the PPROMEXILtrial). BMC pregnancy and childbirth 7:11 (6 July 2007) 
OTHER PUBLICATIONS

J Langenveld, ACJ Ravelli, AH van Kaam, DP van der Ham, MG van Pampus, M Porath, BWJ Mol, W Ganzevoort. Neonatal outcome of pregnancies complicated by hypertensive disorders between 34 and 37 weeks of gestation: a 7-year retrospective analysis of a national registry. Am J Obstet Gynecol. 2011 Dec. 205(6); 504.e1-7. Epub 2011 Jul 20

ASP van Teeffelen, DP van der Ham, SG Oei, M Porath, C Willekes, BWJ Mol. The accuracy of clinical parameters in the prediction of perinatal pulmonary hypoplasia secondary to midtrimester prelabour rupture of fetal membranes: a meta-analysis. Eur J Obstet Gynecol Reprod Biol. 2010 Jan;148(1):3-12. Epub .

DP van der Ham; R van de Laar; BWJ Mol; C Willekes. Letter to the editor (on TrochezMartinez; BJOG 2007;114:796-801). BJOG 2008 Jan;115(1):127; author reply 128

DP van der Ham. Myasthenia gravis en zwangerschap. NTOG vol 119 no 4. Mei 2006; 13-15.

DP van der Ham, JK de Vries, PL Van der Merwe. Mitral Valve Prolapse: a study of 45 children. Cardiovascular Journal of South Africa: vol 14 no 4. July/August 2003; 191-194

\section{ORAL PRESENTATIONS}

DP van der Ham, of behalf of the PPROMEXIL trial group. Induction of labor versus expectant management in women with preterm prelabor rupture of membranes between 34 and 37 weeks - the PPROMEXIL-2 trial. 32nd Annual Meeting of the Society of Maternal-Fetal Medicine, Dallas, USA. February $9^{\text {th }} 2011$

DP van der Ham, of behalf of the PPROMEXIL trial group. Antepartum predictie van neonatale sepsis bij vrouwen met PPROM tussen 34 en 37 weken. Gynaecongres, Sessie: In opleiding 1 - Breda. June $4^{\text {th }} 2010$

DP van der Ham, of behalf of the PPROMEXIL trial group. PPROMEXIL - Induction of Labour versus Expectant management in women with Preterm Prelabour Rupture of Membranes between 34 and 37 weeks - eerste resultaten. Gynaecongres, Sessie: Nieuws van eigen bodem - Papendal. November $12^{\text {th }} 2009$

DP van der Ham, R van de Laar, JJ van Beek, C Willekes, BWJ Mol. Accuracy of C-reactive 
protein in predicting chorioamnionitis and neonatal sepsis in women with premature rupture of membranes: A systematic review 35th Annual Meeting of the Fetal and Neonatal Physiological Society, Maastricht. June $24^{\text {th }} 2008$

\section{POSTER PRESENTATIONS}

DP van der Ham, BWJ Mol, JM Schaaf, BM Kazemier, AC Ravelli. Pregnancy outcome of subsequent pregnancy after a history of preterm birth with and without PPROM. 33th Annual Meeting of the Society of Maternal-Fetal Medicine - San Francisco, USA. February $11^{\text {th }}-16^{\text {th }} 2013$.

DP van der Ham on behalf of the PPROMEXIL study group. Prediction of neonatal sepsis in women with PPROM between 34 and 37 weeks of gestational age. 31th Annual Meeting of the Society of Maternal-Fetal Medicine - San Francisco, USA. February $7^{\text {th }}-12^{\text {th }} 2011$.

DP van der Ham on behalf of the PPROMEXIL study group. Does induction of labor in women with a positive GBS vaginal culture decrease the risk for neonatal sepsis in women with PPROM between 34 and 37 weeks? 31th Annual Meeting of the Society of Maternal Fetal Medicine - San Francisco, USA. February $7^{\text {th }}-12^{\text {th }} 2011$.

DP van der Ham on behalf of the PPROMEXIL trial group. Induction of Labor Versus Expectant Management in Women with Preterm Prelabor Rupture of Membranes between 34 and 37 Weeks - the PPROMEXIL trial - 57th Annual Scientific Meeting of the Society of Gynecological Investigation - Orlando, USA. March 22 $2^{\text {nd }}-27^{\text {th }} 2010$.

DP van der Ham, J van der Heijden, ACJ Ravelli, JG Nijhuis, A Mulder, J J van Beek, BWJ Mol, C Willekes. Neonatal outcome of pregnancies complicated by PPROM between 34 and 37 weeks of gestation. 30th Annual Meeting of the Society of Maternal Fetal Medicine - Chicago, USA. February $1^{\text {st }-6 \text { th }} 2010$

DP van der Ham, JJ van Beek. Outcome of conservative laparoscopic surgery in advanced endometriosis: recurrence and consequences for infertility. 15th Annual Congress of the European Society for Gynaecological Endoscopy, Strasbourg, France. October $5^{\text {th }} 7^{\text {th }} 2006$

DP van der Ham, JJ van Beek. Predictive factors for recurrence of complaints and/or endometriotic implants after surgical treatment of endometriosis. IX World Congress on Endometriosis - Maastricht (World Endometriosis Society). September $14^{\text {th }}-17^{\text {th }} 2005$ 


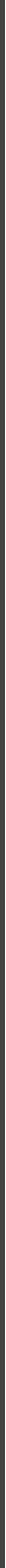


DANKWOORD 


\section{DANKWOORD}

Dagen, nachten, weken, maanden zwoegen en eindelijk na ruim zes jaar is het af. Met veel plezier heb ik de afgelopen jaren gewerkt aan de PPROMEXIL studie en de afronding van dit proefschrift. Promoveren doe je echter niet alleen en velen ben ik dan ook dank verschuldigd voor hun inzet.

Allereerst wil ik alle zwangere vrouwen en hun partners die belangeloos deelnamen aan de PPROMEXIL studie bedanken. Ze werden allen geconfronteerd met het breken van de vliezen voor de uitgerekende datum. Zij zagen het nut van hun deelname aan wetenschappelijk onderzoek naar deze zwangerschapscomplicatie in. Zonder hun inzet was dit proefschrift er niet geweest. Bedankt!

Prof. dr. B.W.J. Mol, beste Ben Willem, waar moet ik beginnen? Het begin, 2006, je kwam een presentatie houden in Venlo waar ik net aan mijn opleiding was begonnen. Je bevlogenheid en enthousiasme waren en zijn nog steeds inspirerend. Toen er niet lang daarna gezocht werd naar een promovendus voor een gebroken vliezen studie hoefde ik niet lang na te denken om hierop ja te zeggen. Ik voel me vereerd dat ik een van je promovendi mag zijn.

Je hebt me gemotiveerd om mezelf verder te ontwikkelen. Bij mijn vraag hoe ik een predictiemodel moest maken was je antwoord: "Met een multivariabele logistische regressie..." om hieraan toe te voegen, voordat ik iets kon zeggen "... dat kun je wel." Het kostte mij een week, maar ... het lukte.

Het heeft nooit aan jou gelegen dat iets langer duurde dan gepland, soms bijna nog voordat ik de knop "verzenden" had ingedrukt kreeg ik alweer antwoord op mijn e-mail. Een aparte ringtone voor jou was ook niet nodig, als ik op een onmogelijk tijdstip werd gebeld, kon het maar één iemand zijn. Het is je vergeven.

Jouw persoonlijke betrokkenheid tijdens het ziekbed van mijn vader en je inzet om de promotiedatum te vervroegen, hetgeen uiteindelijk niet haalbaar bleek te zijn, hebben mij, Gineke en mijn ouders geraakt en goed gedaan.

Prof. dr. J.G. Nijhuis, beste Jan, halverwege het PPROMEXIL project haakte je aan als een van mijn promotoren. Je helikopterview was vaak heel verhelderend. Menig keer heb je tegen mij gezegd: "Een proefschrift is een proeve van wetenschappelijk onderzoek en het begin van een wetenschappelijke carrière, niet je levenswerk." Toch moest in mijn beleving dit proefschrift een kop, een lijf en een staart hebben. Het beestje is nu af. Beste Jan, dank voor je kritische blik, medeleven en support. 
Dr. C. Willekes, beste Christine, "Zeg maar Christine en geen u" waren volgens mij je eerste woorden toen wij elkaar voor het eerst telefonisch spraken. Ik was aan je gekoppeld als je eerste promovendus. Je laagdrempeligheid was fijn en samen begonnen we aan dit avontuur. Je bevlogenheid voor je patiënten en het echografisch onderzoek was en is bijzonder, je hebt twee prachtige 20 weken echo sessies gemaakt van Sophie en Jasmijn. Mijn zelfredzaamheid en jouw passie voor patiëntenzorg maakten soms dat onze koersen wat uit elkaar dreven Toch ben ik ervan overtuigd dat we het avontuur goed hebben volbracht. Bedankt!

Dr. A.L.M. Mulder, beste Twan, graag had jij de verslaglegging en beoordeling van de neonaten prospectiever gezien. Helaas moesten we roeien met de riemen die we hadden (te korte riemen; lees te weinig budget). Desondanks heb je samen met Rob Moonen ontzettend veel werk verzet om alle neonatale statussen te scoren op sepsis. Ik kon altijd bij je aankloppen voor je advies en commentaar. De invalshoek vanuit de neonatologie was vaak erg verhelderend en onontbeerlijk in onderzoek waarbij het kind de belangrijkste uitkomst is.

Dr. J.J. van Beek, beste Hans, als jij het niet in mij had zien zitten was dit proefschrift er mogelijk niet eens geweest. Na samen gewerkt te hebben aan een retrospectief onderzoek naar endometriose in Zwolle, nam je mij, als opleider in het VieCuri Medisch Centrum in Venlo, aan voor de opleiding gynaecologie. Toen kort na het begin van mijn opleiding in Venlo Ben Willem Mol naar een promovendus zocht schoof jij mij naar voren. Hoewel de verloskunde niet meer je aandachtsgebied is, ben je al die jaren de progressie van de PPROMEXIL studie en dit proefschrift aan de zijlijn blijven volgen. Onder jouw supervisie zette ik mijn eerste stappen in de laparoscopie. Naast de verloskunde ligt mijn passie mede dankzij jouw begeleiding bij de minimaal invasieve en ambulante gynaecologie.

Dr. B.C. Opmeer, beste Brent, hartelijk dank voor al je inzet en uitleg. Als ik weer eens vast liep in een statistische bewerking in SPSS kon ik altijd bij je terecht. Hoewel ons contact vrijwel alleen uit e-mails bestond, maakte jij de afgelopen jaren altijd deel uit van het team.

Beste Prof. Dr. C.A..Bruggeman, Prof. Dr. J.A.M. van der Post, Dr. P.H. Dijk, Prof. Dr. L.J.I. Zimmerman, Prof. Dr. F.C.S. Ramaekers, als leden van de beoordelingscommissie, hartelijk dank voor het kritisch beoordelen van mijn manuscript

Beste PPOMEXIL projectgroep leden en alle coauteurs, bedankt voor jullie hulp bij alle 
inclusies en kritische beoordelingen van de manuscripten. Ik denk dat we met z'n allen trots mogen zijn op het behaalde resultaat.

Kim, Corine, Arianne, Michelle, Carolien, collega arts-onderzoekers van het eerste uur en alle andere arts-onderzoekers van het consortium, bedankt voor de leuke en leerzame bijeenkomsten in Utrecht, Castle Course in Frankrijk en Veldhoven. Wie had kunnen denken dat de kleine groep waarmee we begonnen inmiddels is uitgegroeid tot zijn huidige omvang.

Beste Maya Kruijt, waar zou ik zijn zonder jou? En natuurlijk zonder de andere medewerkers van het trialbureau (Cynthia Crul en Zelda van Dijk). Al het monnikenwerk wat je/ jullie de afgelopen jaren voor mij en de andere arts-onderzoekers hebt/hebben verricht. De stapels METC aanvragen die je voor mij hebt verzonden, en natuurlijk alle andere hand-en-spandiensten. Zonder jou/jullie was het consortium niet zo groot en goed georganiseerd als dat het nu is.

Maatschap gynaecologie VieCuri Medisch Centrum in Venlo. De eerste drie jaar van mijn opleiding heb ik bij jullie mogen doorbrengen. De omschakeling tussen het Noorden en Limburg verliep niet altijd even gemakkelijk. Toch heb ik veel geleerd en gaven jullie mij de mogelijkheid om gedurende geruime tijd een dag(deel) in de week te gebruiken voor mijn promotieonderzoek, waarvoor dank.

Beste stafleden en junior staf van het Maastricht Universitair Medisch Centrum, dank voor de drie jaar dat ik jullie collega mocht zijn. De academie is zo slecht nog niet. Toch trokken de "roots" me terug naar het Noorden. Beste Prof. dr. R.F.P.M Kruitwagen, beste Roy en dr. G.A.J. Dunselman, beste dokter D, dank voor jullie medewerking in het realiseren van de eerste differentiatiestage van het cluster Maastricht en dan ook nog eens direct buiten het cluster.

Maatschap gynaecologie Martini Ziekenhuis in Groningen. Het voelde en voelt nog steeds als thuiskomen. Beste Marinus en Aren, dank dat jullie mij de gelegenheid gaven om de sprong te wagen en een differentiatie stage bij jullie te komen doen. Hoewel het bijna nog misgelopen was ... maar net op tijd is ook op tijd, of niet Marinus? Ik voel me bij jullie als een vis in het water, Aren, Sjaak, Yenny, Truus, Marinus, Britt, Niels, Jorien en Fleurisca, ik hoop dat onze samenwerking nog lang mag blijven duren. En Niels dank voor je tip, ik vind het goed gelukt, jij? 
Verpleegkundigen, verloskundigen, kraamverzorgende, polimedewerkers in het VieCuri, MUMC en het Martini Ziekenhuis, dank voor de samenwerking in de afgelopen jaren en jullie gezelligheid in de (nacht)diensten. Met inmiddels drie prachtige dochters en dito bevallingen in het ziekenhuis is het meer dan eens duidelijk, zonder jullie inzet en ondersteuning gaat het niet!

Lieve, vrienden en familie wat fijn dat jullie er zijn in goede en in slechte tijden.

Lieve "geneeskunde" vrienden, Jeroen, Marjan, Jelleke, Michel en Annemieke, wie had dat kunnen denken, sinds het begin van de studie in 1998 trokken we vaak samen op en nog steeds zien wij elkaar geregeld. De groep is alleen groter geworden met Michelle, Duncan en Geert en inmiddels 9 kinderen. Ik verheug me nu al op het komende tig-gangen kerstdiner en het volgende dagje "pretpark".

Mijn paranimfen, Stijn en Nynke. Beste Stijn, helaas is het niet gelukt om op dezelfde dag te promoveren, het was misschien ook wel te ambitieus. Toch ben ik erg blij dat je mijn paranimf wil zijn. Een onvergetelijke trip naar Dallas (SMFM 2012) en een promotie onderzoek die in elkaars verlengde ligt, leidde tot een goede samenwerking, maar inmiddels ook een goede vriendschap. Nu op naar jouw promotie!

Lieve Nynke, mijn "halfzus", wat fijn dat jij mijn paranimf wil zijn. Hoewel we elkaar de afgelopen jaren door reisafstand en werk soms maanden niet zien, voelt ieder bezoek weer of we elkaar gisteren voor het laatst hebben gezien.

Lieve schoonfamilie, sorry voor mijn ongezellige momenten als ik weer eens aan de keukentafel bezig was met onderzoek tijdens een familiebezoek vanuit Venlo. Desondanks waren jullie altijd geïnteresseerd en leefden mee met alle hoogte en menig dieptepunt (bij weer een afwijzing van een tijdschrift). Groningse nuchterheid in golden raand. Jannes, het duurde 10 jaar voordat jij je promotie had afgerond, ik zou het een stuk sneller doen heb ik altijd geroepen ... maar toch heel veel sneller werd het ook niet.

Marco en Christiaan, lieve broers, hoewel onze interesses totaal verschillend zijn, ben ik blij dat jullie mijn broer(tje)s zijn.

Lieve mama, lieve papa, het kan niet anders dan jullie samen te bedanken. Bedankt dat ik nadat ik uitgeloot was voor geneeskunde een jaar Engels kon gaan studeren in Hastings. Zonder dat jaar was dit proefschrift nooit gelukt.

Mijn promotietraject hebben jullie letterlijk en figuurlijk op een afstand gevolgd. Wat pro- 
moveren precies inhoud was soms moeilijk uit te leggen, maar jullie waren altijd geïnteresseerd.

Na zes jaar Venlo kwamen we begin 2012 "eindelijk" weer terug naar het Noorden. Wat was/is het fijn om zomaar eventjes langs te gaan, de kinderen te laten logeren als wij het weer eens te druk hadden. Lieve papa, helaas heeft dit geluk nog geen jaar mogen duren. Begin februari werd bij jouw uitgezaaide longkanker vastgesteld. Een vervroegde promotie tijdens je ziekbed zag je zelf niet zitten, daarvoor was de kanker te agressief. Op 13 april 2013 een dag voor de geboorte van je derde kleinkind hebben we afscheid van je moeten nemen. Je enorme openheid over je ziekte was bewonderenswaardig, ik hoop dat ik, al is het maar een klein beetje, iets van die openheid mag overnemen. Papa, ik weet zeker dat je trots zou zijn geweest. Ik mis je.

Lieve mama, wat ben ik trots op jou. Ondanks het enorme gemis en de leegte die er nu is, kijk je vooruit. Je bent een trotse moeder en oma.

Lieve Gineke, wat ben ik bevoorrecht dat jij al bijna 13 jaar bij me bent. Mijn dank aan jou is meer dan ik hier kwijt kan. Je hebt me gesteund door dik en dun, je bent met mij mee gegaan naar Venlo om mijn droom om gynaecoloog te kunnen worden te verwezenlijken. Je gaf me alle mogelijkheden om aan dit proefschrift te kunnen werken, waarbij je je menig keer zelf wegcijferde. Daarnaast ben je denk ik de enige die alles van dit proefschrift heeft gelezen en waar nodig, en dat was nog al eens nodig, gecorrigeerd. Eindelijk is het af. Ik ga je geen belofte doen dat nu alle tijd voor jou is, want dan weet je dat ik lieg. Maar het moeten wordt nu mogen ... en ons gezin staat nu echt op één.

Lieve Sophie, Jasmijn en Fleur, wat zijn jullie een verrijking in mijn leven. Jullie foto's zijn voor mij het hoogtepunt van dit proefschrift! 



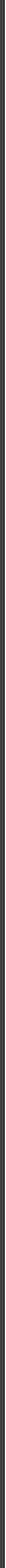




\section{CURRICULUM VITAE}





\section{CURRICULUM ViteA}

David Pelaja van der Ham werd op 15 november 1978 geboren te Groningen. Hij groeide op in Drachten, waar hij in 1997 aan het c.s.g. Liudger zijn VWO diploma behaalde.

Na een jaar in Hastings (Groot-Britannië) op een internationale school Engels te hebben gestudeerd, startte hij in 1998 met de studie geneeskunde aan de Rijksuniversiteit Groningen. Zijn wetenschappelijke stage deed hij in 2002 naar mitral valve prolaps in children aan de Universiteit van Stellenbosch in het Tygerberg Hospital te Kaapstad onder leiding van prof dr. P.L. van der Merwe.

Vervolgens liep hij zijn co-schappen in het Scheperziekenhuis te Emmen. In 2004 liep hij in de Isala Klinieken te Zwolle zijn keuze-coschap gynaecologie en ging aansluitend als ANIOS gynaecologie hier aan de slag (opleider dr. H.H. de Haan). In 2006 begon hij aan de opleiding tot gynaecoloog in het VieCuri Medisch Centrum te Venlo met als opleider $\mathrm{dr}$. J.J. van Beek. In 2007 startte hij naast zijn opleiding als promovendus met de PPROMEXILstudie onder leiding van prof. dr. B.W. Mol en prof. dr. J.G. Nijhuis. In 2009 begon hij zijn academische deel van de opleiding tot gynaecoloog in het Maastricht Universitair Medisch Centrum te Maastricht (opleiders prof. dr. G.G.M. Essed en later prof. dr. R.F.P.M. Kruitwagen en vice-opleider G.A.J..Dunselman). In 2012 heeft hij met een differentiatiestage algemene en minimaal invasieve gynaecologie in het Martini Ziekenhuis te Groningen (opleider dr. A.J. van Loon) zijn opleiding tot gynaecoloog afgerond. Sinds 1 januari 2013 is hij als chef de clinique gynaecologie werkzaam in het Martini Ziekenhuis te Groningen.

In 2000 leerde David Gineke tijdens zijn studie kennen, met wie hij in 2007 trouwde. In 2008, 2011 en 2013 werden zij trotse ouders van Sophie, Jasmijn en Fleur. 




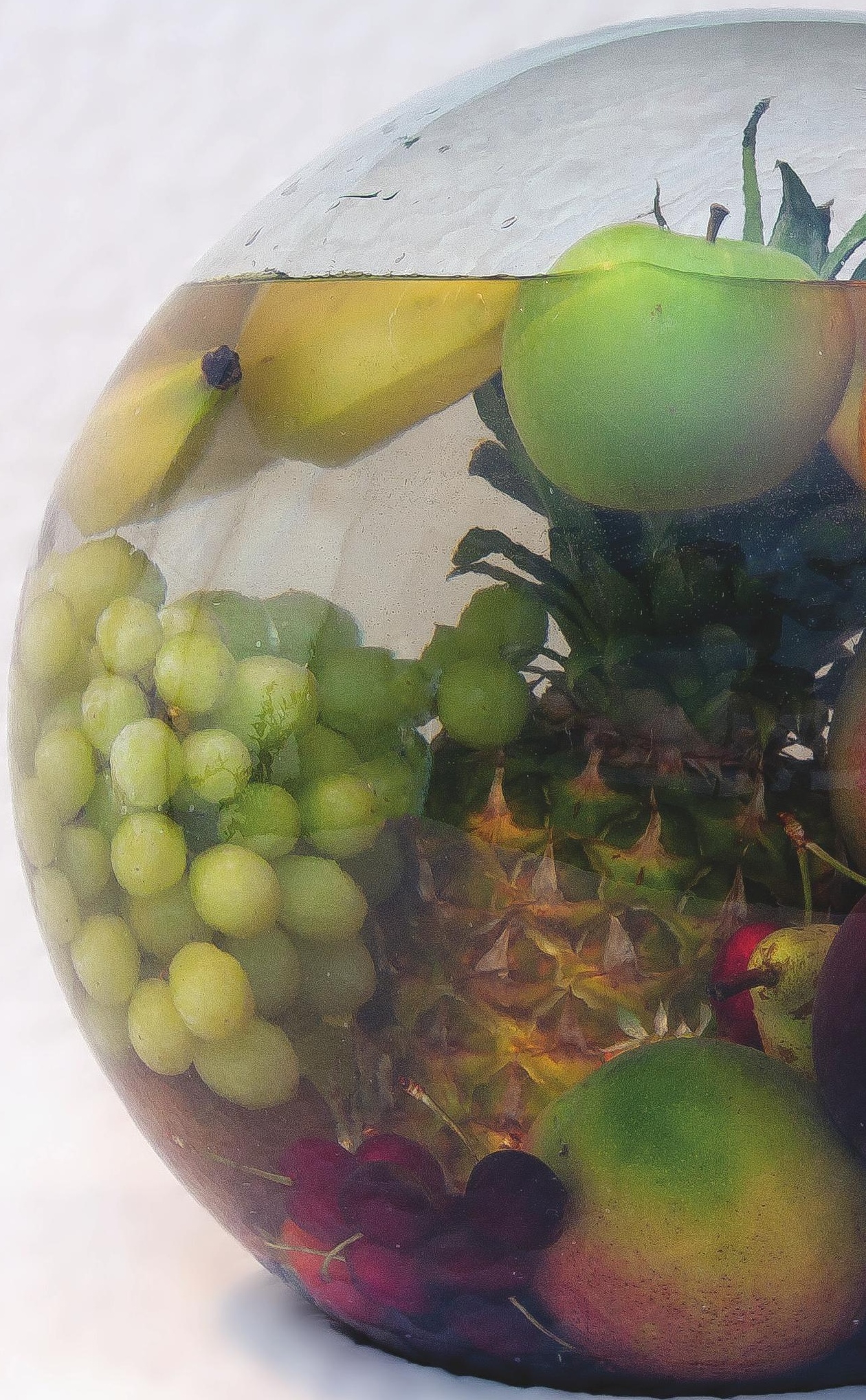

